\title{
Enabling the Blind to See Gestures
}

\author{
Francisco CMB Oliveira
}

Dissertation Proposal submitted to the faculty of Virginia Polytechnic Institute and State University in partial fulfillment of the requirements for the degree of

\author{
Doctor of Philosophy \\ in \\ Computer Science
}

\author{
Francis Quek, Chair \\ Deborah Gail Tatar \\ Steve Harrison \\ Dennis Gracanin \\ Tonya Smith-Jackson
}

August $2^{\text {nd }}, 2010$

Blacksburg, Virginia

Keywords: Multimodal interfaces, Situated discourse, Embodied deictic activity, Common Ground, Electronically Mediated Communication

Copyright 2010, Francisco Carlos de Mattos Brito Oliveira 



\title{
Enabling the Blind to See Gestures
}

\author{
Francisco Carlos de Mattos Brito Oliveira
}

(ABSTRACT)

Mathematics instruction and discourse typically involve two modes of communication: speech and graphical presentation. For the communication to remain situated, dynamic synchrony must be maintained between the speech and dynamic focus in the graphics. Sighted students use vision for two purposes: access to graphical material and awareness of embodied behavior. This embodiment awareness keeps communication situated with visual material and speech. Our goal is to assist students who are blind or visually impaired (SBVI) in the access to such instruction/communication. We employ the typical approach of sensory replacement for the missing visual sense. Haptic fingertip reading can replace visual material. We want to make the SBVI aware of the deictic gestures performed by the teacher over the graphic in conjunction with speech. We employ a haptic glove interface to facilitate this embodiment awareness. In this research, we address issues from the conception through the

design, implementation, evaluation to the effective and successful use of our Haptic Deictic System (HDS) in inclusive classrooms.

\section{GRANT INFORMATION}

This research has been supported by:

The National Science Foundation HSD program, Grant \# ISS-0451843, and by CNPq (Conselho Nacional de Desenvolvimento Científico e Tecnólogico - Brasil) 



\section{Dedication}

To my beloved wife, Viviany

To my children, Pedro and Lígia

To my parents, Antônio Carlos and Elsa

To Luíza (Di), Raimundo and Cilda

To Michele, Moacir, João and Malu

To Francis Quek

To all my friends in Blacksburg 



\section{Contents}

\begin{tabular}{lll}
\hline & Introduction & 1
\end{tabular}

1.1 Introduction . . . . . . . . . . . . . . . . . . . . . 3

1.2 Language, gesture and thought . . . . . . . . . . . . . . . . . . 4

1.2.1 Gesturing and mathematical thought . . . . . . . . . . . . . . 5

1.3 Gesture, Social interaction, Teaching and Learning . . . . . . . . . . . . . 6

1.4 Pointing . . . . . . . . . . . . . . . . . . . . . . 8

1.4 .1 Gesturing, environment and culture . . . . . . . . . . . . . . . . 11

1.4 .2 Pointing combined with description . . . . . . . . . . . . . . . . 11

1.5 Teaching the blind $\ldots \ldots \ldots \ldots$. . . . . . . . . . . . . . . 13

$1.6 \quad$ Embodiment Awareness and Cognition . . . . . . . . . . . . . . . . . . . 16

1.7 Transition . . . . . . . . . . . . . . . . . . . . . 18

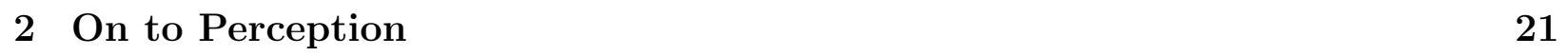

2.1 On Perception . . . . . . . . . . . . . . . . . . . . . . . . . . 23

2.2 Uses of tact to acquire useful information . . . . . . . . . . . . . . . . 25

2.2 .1 Haptics in math teaching for the blind . . . . . . . . . . . . . . 27

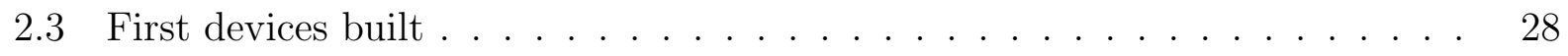

2.3 .1 The reverse joysticks . . . . . . . . . . . . . . . . . . . . . . . . . 29

2.3 .2 The choice for the hands for conveying tactile stimuli . . . . . . . . . 30

2.3 .3 Other Haptic Gloves . . . . . . . . . . . . . . . . . . . . . . . . . . . 32

2.3 .4 Initial models of our haptic gloves . . . . . . . . . . . . . . . 36 
2.3 .5 Tactile perception threshold $\ldots \ldots \ldots \ldots$. . . . . . . . . . 37

$2.3 .6 \quad$ How to convey the Displacement Vector $-D V \ldots \ldots \ldots \ldots$

2.4 The Experiments $\ldots \ldots \ldots \ldots$

$2.4 .1 \quad$ Experiment $1 \ldots \ldots \ldots \ldots$

2.4 .2 Tracking . . . . . . . . . . . . . . . . . . . . . . . . . 45

2.4 .3 Experiment II $\ldots \ldots \ldots \ldots \ldots$

$2.4 .4 \quad$ Experiment III $\ldots \ldots \ldots \ldots \ldots \ldots \ldots$

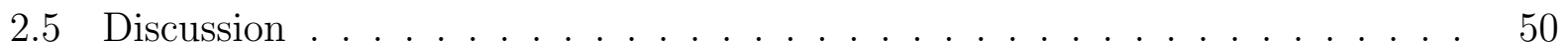

2.6 The need for new glove models . . . . . . . . . . . . . . . . . . . 52

$2.6 .1 \quad$ Revisiting the signaling strategy $\ldots \ldots \ldots \ldots \ldots$

2.7 Transition . . . . . . . . . . . . . . . . . . . . . . . . . . 55

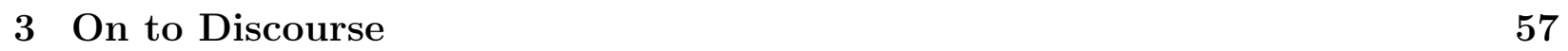

$3.1 \quad$ Introduction . . . . . . . . . . . . . . . . . . . . . . . . . . . 59

3.2 The Haptic Deictic System - HDS $\ldots \ldots \ldots$. . . . . . . . . . . . . . . . . . 60

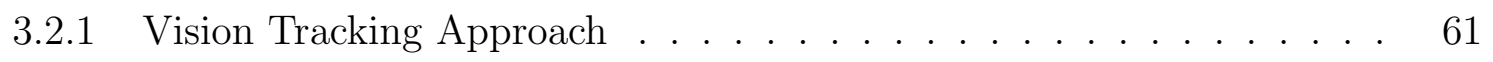

3.2 .2 The haptic aided navigation subsystem . . . . . . . . . . . 65

3.2 .3 The Instructor's Display $\ldots \ldots \ldots$

3.3 Common Ground $\ldots \ldots \ldots$. . . . . . . . . . . . . . . . . . 66

3.4 How the game is played $\ldots \ldots \ldots \ldots \ldots$. . . . . . . . . . . . . . . 72

$3.4 .1 \quad$ Similarities between the charade game and instruction $\ldots . . .73$

$3.5 \quad$ Recruiting Participants Who are Blind $\ldots \ldots \ldots \ldots$. . . . . . . . 74

3.6 The trials $\ldots \ldots \ldots \ldots \ldots \ldots$

3.7 Guides' and Followers' Experiences $\ldots \ldots \ldots$. . . . . . . . . 76

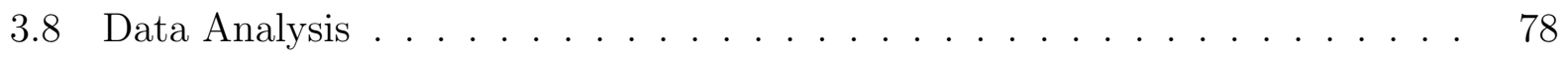

3.9 Lessons learned $\ldots \ldots \ldots$. . . . . . . . . . . . . . . . . . . . . . . 85

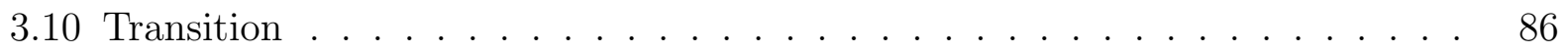

\begin{tabular}{|lll}
4 & Enabling Embodied Skill through Game Playing & 87
\end{tabular} 
4.1 Introduction $\ldots \ldots \ldots \ldots$

4.2 Games and Accessibility . . . . . . . . . . . . . . . . . . . 90

4.3 Designing for flow Experience . . . . . . . . . . . . . . . . . . . . . 92

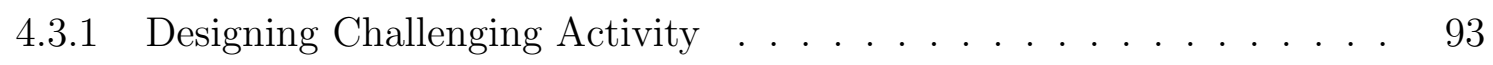

4.3 .2 The Flexible Game Structure $\ldots \ldots \ldots \ldots$

4.3 .3 The game levels $\ldots \ldots \ldots \ldots$

4.4 The trials $\ldots \ldots \ldots \ldots$

4.5 The Gamers' Experience $\ldots \ldots \ldots \ldots$

4.6 Data Analysis $\ldots \ldots \ldots \ldots$

4.7 Revisiting the Phrase Charade . . . . . . . . . . . . . . . . . . . 103

4.7 .1 Performance Gains and Task Focus . . . . . . . . . . . . . . . 104

4.7 .2 Comparing Charade Game Experience . . . . . . . . . . . . . 105

4.8 Transition $\ldots \ldots \ldots \ldots$

5 On to Instruction 109

5.1 Introduction . . . . . . . . . . . . . . . . . . . . . . 111

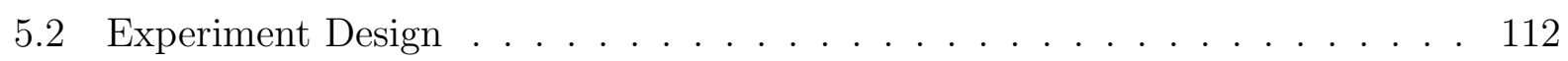

5.2 .1 The Participants $\ldots \ldots \ldots \ldots \ldots$

5.3 Data Analysis . . . . . . . . . . . . . . . . . . . . . . . . . . . . . . . . 114

$5.3 .1 \quad$ Challenges . . . . . . . . . . . . . . . . . . . . . . . . 114

5.3 .2 Analysis Instruments $\ldots \ldots \ldots \ldots$

5.3 .3 Detailed Analysis and Measures . . . . . . . . . . . . . . . . . . . . 119

5.3 .4 Instructors' and Student's Experience . . . . . . . . . . . . . . 145

5.4 Overall conclusions from the Instruction Study . . . . . . . . . . . . . . . 149

5.5 New Opportunities $\ldots \ldots \ldots \ldots \ldots$

5.6 Summary of the Work Completed $\ldots \ldots \ldots \ldots \ldots$

\begin{tabular}{|lr}
\hline A Chapter 2 - Additional Material & 177
\end{tabular}

A.1 Confusion Matrix . . . . . . . . . . . . . . . . . . . . . . . . . . . . . 179 
A.2 Vibrating Motor Spec . . . . . . . . . . . . . . . . . . . . . . . . . 181

A.3 Initial Studies Demographic Questionnaire . . . . . . . . . . . . . . . 183

A.4 Initial Studies Post Questionnaire . . . . . . . . . . . . . . . . . . 185

\begin{tabular}{ll}
\hline B Chapter 3 - Additional Material & 189
\end{tabular}

B.1 Catch Phrase Survey . . . . . . . . . . . . . . . . . . . . . . . . . . . . 191

B.2 Clue phrases questionnaire . . . . . . . . . . . . . . . . 193

B.3 Blind follower post-questionnaire . . . . . . . . . . . . . . 196

B.4 The answers the followers who are blind gave to their post-questionnaire . . 204

B.5 Guide's post-questionnaire . . . . . . . . . . . . . . . . . . . . . 208

B.6 The guides' answers to their postquestionnaire . . . . . . . . . . . 215

\begin{tabular}{ll}
\hline C Chapter 4 - Additional Material & 217
\end{tabular}

C.1 Game-Pre questionnaire . . . . . . . . . . . . . . . . . . . . 222

C.2 Game-Post questionnaire . . . . . . . . . . . . . . . . . 225

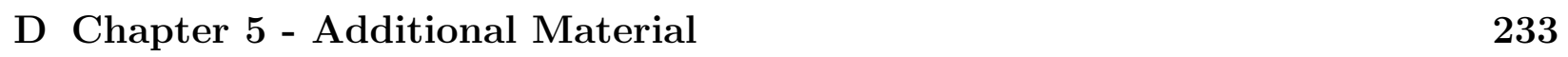

D.1 Curriculum A . . . . . . . . . . . . . . . . . . . . . . . . . . . 235

D.1.1 First Lesson . . . . . . . . . . . . . . . . . . . . . . . . . . . . . . . 235

D.1.2 Second Lesson . . . . . . . . . . . . . . . . . . . . . . . . . 237

D.1.3 Third Lesson . . . . . . . . . . . . . . . . . . . . . . . . . . . . . . . 239

D.2 Pre-test: Curriculum A . . . . . . . . . . . . . . . . . . . . . . . . . 241

D.3 Post-test: Curriculum A . . . . . . . . . . . . . . . . . . . . . . . . 242

D.4 Curriculum B . . . . . . . . . . . . . . . . . . . . . . . . . . . . . . . . 243

D.4.1 First lesson . . . . . . . . . . . . . . . . . . . . . . . . . . . . . . 243

D.4.2 Second Lesson . . . . . . . . . . . . . . . . . . . . . . 246

D.4.3 Third lesson . . . . . . . . . . . . . . . . . . . . . . . . . . . . . . 248

D.5 Pre-test: Curriculum B . . . . . . . . . . . . . . . . . . . . . 251

D.6 Post-test: Curriculum B . . . . . . . . . . . . . . . . . . . . . . . . . . 252

D.7 Student Demographic Questionnaire . . . . . . . . . . . . . . . . 253 
D.8 Teacher Demographic Questionnaire . . . . . . . . . . . . . . . . . . . 254 



\section{List of Figures}

$1.1 \quad$ Seven different ways of pointing, from Kendon [154], page 206 $\ldots . .9$

1.2 Pointing and description $\ldots \ldots \ldots \ldots$. . . . . . . . . . . . . . . . 12

2.1 The reverse joysticks. . . . . . . . . . . . . . . . . . . . . . 30

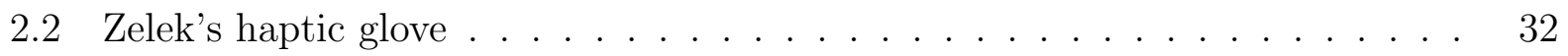

$2.3 \quad$ Multimodal graphic exploration proposed by Manshad and Manshad [180] . 33

2.4 First and second generations of haptic gloves. . . . . . . . . . . . . . . . . 37

2.5 A Norwegian newspaper cartoonist's illustration of "sensory saltation" [87]. . 40

2.6 A possible way to convey North using 5 x 4 glove . . . . . . . . . . . . . . 41

$2.7 \quad$ Experiment I - A participant choosing the direction he perceived . . . . . . . 43

2.8 Charts from Experiments I and II . . . . . . . . . . . . . . . . . . . . . 45

2.9 Focal Disparity (direction) computed from TPA and PIF . . . . . . . . . . . 46

2.10 Charts from Experiment III . . . . . . . . . . . . . . . . . . . . . . 49

2.11 Locations of the receptive field centers of 334 globrous skin mechanoreceptive units, from Johansson [146] f . . . . . . . . . . . . . . . . . . . . 51

2.12 How the vibrating motors were arranged on the palm of the hand and the "Stretchable" glove. . . . . . . . . . . . . . . . 53

2.13 The fourth and latest glove model. . . . . . . . . . . . . . . . . . 54

3.1 The Haptic Deictic System - HDS . . . . . . . . . . . . . . . . . . . . . . . 60

3.2 Points of Instructional Focus and SBVI Tactile Access . . . . . . . . . . . . . 61

3.3 Background subtraction for PIF . . . . . . . . . . . . . . . . . . 62

3.4 Color codes for the instructor's display $\ldots$. . . . . . . . . . . . . . . . . 66 
3.5 Puzzles containing the clue phrases $\ldots \ldots$. . . . . . . . . . . . . . . . 72

3.6 Introducing a sine wave . . . . . . . . . . . . . . . . . . . . . 74

3.7 Cameras to record the trials from different perspectives . . . . . . . . . . . . 78

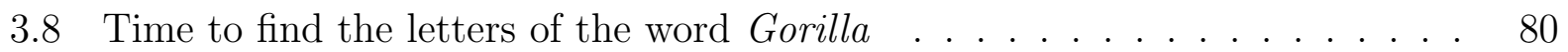

4.1 The game for blind . . . . . . . . . . . . . . . . . . . . . . . . 94

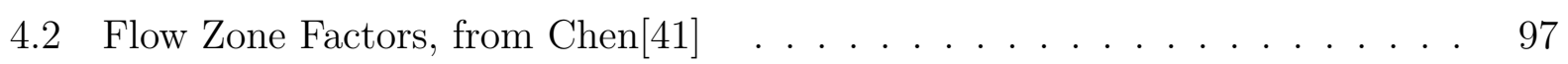

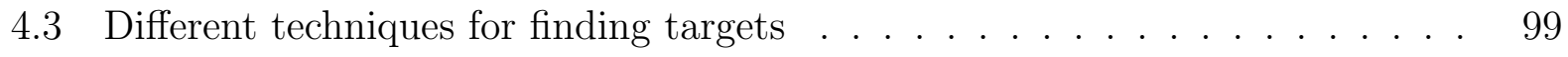

4.4 Percentage of Conversational Turns Dedicated to Solving the Charade - before and after playing the Mission Impossible Game . . . . . . . . . . . . . . . . 105

5.1 A and B has the same measure. C and D also has the same measure. . . . . 124

5.2 Transversal line creates congruent angles when it intersects parallel lines . . 128

5.3 A circle with its center, diameter, radius and circumference identified . . . . 132

5.4 Words per conversational turn (Instructor) - Overall Comparison . . . . . . 135

5.5 Turn duration (Instructor) - in seconds . . . . . . . . . . . . . . . . . . 137

5.6 Hand positioning events . . . . . . . . . . . . . . . . . . . . 139

5.7 Focus on lesson's objectives - Overall comparison . . . . . . . . . . . . . . . 140

5.8 Deictic Expressions - Overall comparison . . . . . . . . . . . . . . . . . . . . 142

5.9 Need to do cognitive offloading - "O" is writing on her palm . . . . . . . . . 152

A.1 Vibrating motor spefication sheet . . . . . . . . . . . . . . . . 182

D.1 Angle of the sun's rays . . . . . . . . . . . . . . . . . . . . . 235

D.2 Sun's rays assumed to be parallel . . . . . . . . . . . . . . . . . . . . 236

D.3 Euclid's Proposition 33 . . . . . . . . . . . . . . . . . . . . . 237

D.4 Finding the length of the circle's circumference . . . . . . . . . . . . . . . . . 238

D.5 Euclid's Proposition 33 . . . . . . . . . . . . . . . . . . . . . . . . . . . . 240

D.6 Right Triangle . . . . . . . . . . . . . . . . . . . . . . . . . . . 243

D.7 Right Triangle - Example . . . . . . . . . . . . . . . . . . . . . . . . . 244

D.8 First Graph . . . . . . . . . . . . . . . . . . . . . . . . 246 
D.9 Third Graph . . . . . . . . . . . . . . . . . . . . . . . . . . . . . . . . . . . . 248

D.10 First Graph . . . . . . . . . . . . . . . . . . . . . . . . . . . . . . . 249 



\section{List of Tables}

2.1 Guiard's model of bimanual control (from Mackenzie[177], page 41). . . . . 29

2.2 Two-point Tactile Thresholds, from Christman's work[44], page 386 . . . . . 31

2.3 Core devices used to build haptic displays . . . . . . . . . . . . . . . . . 35

2.4 Response time in seconds per glove model . . . . . . . . . . . . . . . . . . . 44

2.5 Confidence Interval for hit percentages . . . . . . . . . . . . . . . . . . . 44

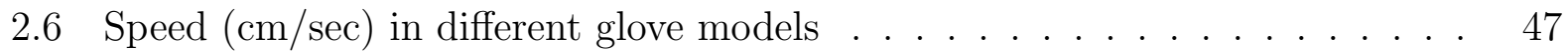

2.7 One of the five stories $\ldots \ldots \ldots$. . . . . . . . . . . . . . . . . . . . 48

2.8 Averages of correct answers by glove model . . . . . . . . . . . . . . . . . . . 49

3.1 Translating PTA coordinates from the student's camera coordinates system to the instructor's camera coordinate system . . . . . . . . . . . . . . . . . . 64

3.2 Clark's Action Ladder . . . . . . . . . . . . . . . . . . . . . . . . . . . . . . 68

$3.3 \quad$ Catch and Clue phrase pairs . . . . . . . . . . . . . . . . . . . . . . 73

3.4 Summary of the trials - Times in seconds . . . . . . . . . . . . . . . . . 79

3.5 Discourse turns to complete the charades . . . . . . . . . . . . . . . 81

3.6 Percentage of object conversational turns . . . . . . . . . . . . . . . . . . . 82

3.7 Expansion turns - times in seconds . . . . . . . . . . . . . . . . . 83

3.8 Clarification questions . . . . . . . . . . . . . . . . . . . . 84

4.1 Game instructions for the first phase . . . . . . . . . . . . . . . . . . 93

4.2 Basic game tuple . . . . . . . . . . . . . . . . . . . . . . . . 95

4.3 Game initial parameters . . . . . . . . . . . . . . . . . . . . . 97

4.4 Navigation Techniques and Time to Target . . . . . . . . . . . . . . . . . . 100 
4.5 Techniques used in second level and Distance Travelled . . . . . . . . . . . . 100

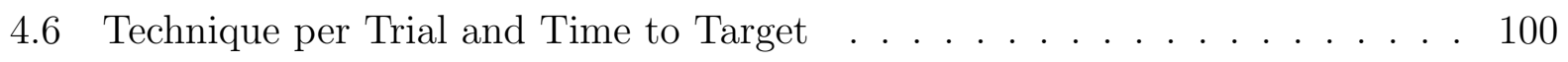

4.7 Trials and Time to Target . . . . . . . . . . . . . . . . . . . . . . . . 101

4.8 Participants and Navigation Techniques . . . . . . . . . . . . . . . . . . . . 101

4.9 Navigation Speeds for game level 2 . . . . . . . . . . . . . . . . . . . . . . . 101

4.10 Participants and distance travelled at game level 2 . . . . . . . . . . . . . 102

4.11 Signaling Patterns and Distance Travelled . . . . . . . . . . . . . . . . . . . 102

4.12 Number of directions and time to target . . . . . . . . . . . . . . . 102

4.13 Number of directions and Navigation Techniques . . . . . . . . . . . . . . . 103

4.14 Catch and Clue phrase pairs . . . . . . . . . . . . . . . . . . . 104

4.15 Blind followers' experience - A comparison between 1st and 2nd charade studies 106

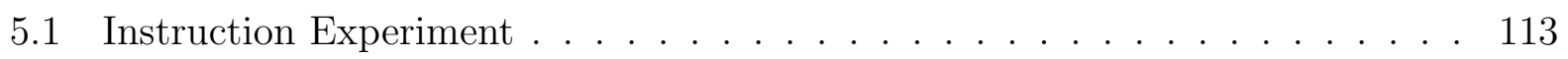

5.2 SBVI participants and when they became blind . . . . . . . . . . . . . . . . 114

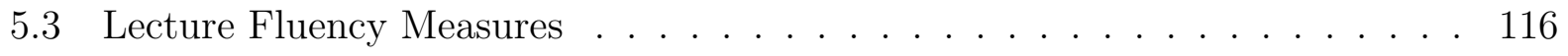

5.4 Words per conversational turn (Instructor) - Overall comparison . . . . . . . 135

5.5 Number of words per conversational turn - Paired Lessons - Curriculum A 136

5.6 Number of words per conversational turn - Paired Lessons - Curriculum B 136

5.7 Turn duration (Instructor) - Overall comparison . . . . . . . . . . . . . . 137

5.8 Average of turn duration - Paired Lessons - Curriculum A . . . . . . . . . 138

5.9 Average of turn duration - Paired Lessons - Curriculum B . . . . . . . . . 138

5.10 Hand positioning (Instructor) - Overall comparison . . . . . . . . . . . . 138

5.11 Hand Positioning Events - Paired Lessons - Curriculum A . . . . . . . 139

5.12 Hand Positioning Events - Paired Lessons - Curriculum B . . . . . . . . . 140

5.13 Focus on lesson's objectives - Overall comparison . . . . . . . . . . . . . . . 141

5.14 Discourse Level - Paired Lessons - Curriculum A . . . . . . . . . . . . . . . . 141

5.15 Discourse Level - Paired Lessons - Curriculum B . . . . . . . . . . . . . . . . 141

5.16 Deictic Expressions - Overall Comparison . . . . . . . . . . . . . . . . . 143

5.17 Percentage of Deictic Turns - Paired Lessons - Curriculum A . . . . . . . . . 143 
5.18 Deictic Turns - Paired Lessons - Curriculum B . . . . . . . . . . . . . . . . . 143

5.19 Summary of the comparisons on Lecture Fluidity Measures . . . . . . . . . . 144

5.20 Post Questionnaires . . . . . . . . . . . . . . . . . . . . . . . . . 145

5.21 Comments Sighted Students' made when comparing attending inclusive classes with and without the system $\ldots \ldots \ldots \ldots \ldots \ldots$

A.1 Confusion Matrix . . . . . . . . . . . . . . . . . . . . . . . . . . . . 180

C.1 Game effect on a deixis-laden discourse . . . . . . . . . . . . . . . . . . 219

C.2 Discourse level analysis . . . . . . . . . . . . . . . . . . . . . . . 220

C.3 Comparing the blind follower's experience between the two charade studies . 221

D.1 Average number of words per conversational turn (Complete Listing) . . . . 302

D.2 Average of turn duration - Instructor (Complete listing) $\ldots . \ldots$. . . . . 303

D.3 Hand Positioning Events (Complete Listing) . . . . . . . . . . . . . . . . . 304

D.4 Discourse Level - Complete listing . . . . . . . . . . . . . . . . . . . . . 305

D.5 Deictic Turns - Complete listing . . . . . . . . . . . . . . . . 306

D.6 Grades per question - Pre-test - Curriculum A . . . . . . . . . . . . 307

D.7 Grades per question - Post-test - Curriculum A . . . . . . . . . . . . . 308

D.8 Grades per question - Pre-test - Curriculum B . . . . . . . . . . . . . . . . . 309

D.9 Grades per question - Post-test - Curriculum B . . . . . . . . . . . . 310 

Chapter 1

\section{Introduction}





\section{$1.1 \quad$ Introduction}

Mathematics instruction and discourse typically involve two modes of communication: speech and graphical presentation. For the communication to remain situated, dynamic synchrony must be maintained between the speech and dynamic focus in the graphics. In normals, vision is used for two purposes: access to graphical material and awareness of embodied behavior of the instructor. This embodiment awareness keeps communication situated between visual material and speech. Unfortunately students who are blind or visually impaired (SBVI) do not have access to such a rich multimodal experience. They cannot resolve the teacher's deictic references towards the instructional material as she lectures [64]. Maybe this is one of the reasons why they are typically one to three years behind their seeing counterparts and are taught in special schools [295]. Our goal is to assist the SBVI in the access to such instruction/communication making it possible for them to attend inclusive classrooms.

We propose a computer system capable of tracking both the teacher's and the student's hands. As in a regular class, the teacher lectures with the help of visual aides. A scaled down raised line version of the shared graphic is available to the SBVI. Static raised line drawings can be cheaply and quickly made, and are easily explored by SBVI [279]. Cameras capture live video from both teacher and student. A haptic glove embedded with vibration haptic actuators tells the student where she must move her hand so that she can keep situated with the information content conveyed by the teacher. We call our system, the Haptic Deictic System - HDS.

Providing a way for the SBVI of understanding the instructor's pointing should lower the grounding costs both in the formulation and understanding of utterances [53], making the lecturing more precise and alleviating the SBVI's cognitive load [48. Such understanding should also improve the student's general awareness of the teacher's embodied behavior and might enhance her engagement in classroom activities. The higher the student's engagement, the better her academic performance [30, 36, 80].

It is expected that both teacher and student will explore new ways of interacting with the $H D S$ introduction 292. Such exploration might change the way mathematics and science are taught to the SBVI. Maybe augmenting the awareness of the teacher's embodied behavior will increase the content uptake by the SBVI. In this dissertation, we investigate how directional information can be conveyed to a student via haptic devices, how to build them so that they are robust, conform to anatomical differences and deliver strong and clear signals. We also investigate what different communication strategies will emerge from the use of such a system and if and how its use lowers grounding costs. This research effort culminates with a study where a mathematics mini course will be taught to different groups of students (SBVI and non-SBVI). Some SBVI participants attend such course with the help of our system, while others are taught in the traditional way. Classroom interactions are assessed through situated video analysis.

In this first chapter, we will discuss how language, gesture and thought are related, and 
the special role they play in mathematics. We then proceed to describe how humans make use of gestures to convey meaning and to understand each other's intentions. Following that, we narrow down the discussion to the teacher/student interactions. The next section addresses one very especial kind of gestures: Pointing. There, we show that hand shape and arm trajectory play important roles in the conveyance of intentions and discuss HDS's limitations and possibilities. We then discuss previous studies on teaching mathematics to an SBVI and speculate how they can inform our research. We finally debate how raising the SBVI's awareness of the teacher can impact her cognitive processes.

\subsection{Language, gesture and thought}

In this section, we will show the deep connection between language, gesture and thought. We will start this discussion by examining the cases of two special persons: Mirabelle and IW.

Mirabelle was born without arms.

Mirabelle:

"When I walk, doctor, my phantoms arms don't swing like normal arms. They stay frozen like this" - her stumps hanging straight down. "But when I talk, my panthoms gesticulate". In fact, they're moving now as I speak." From Ramachandran [221], p 41.

IW lost his sense of touch and proprioception below the neck at the age of nineteen. After thirty years, "he has taught himself to move again using cognition and vision in place of proprioception and spatial position sense", McNeill ([190] p 239). Interestingly, when prevented from seeing, he could not do any instrumental activity. However, in the same condition, he was still able to gesture similar to a normal person.

Both cases, among others, led researchers to conclude that gesture is "not proprioceptive; it is linguistic." McNeill (190] p 245). Furthermore, gestures and signs are interpreted as language by the brain [98. At this point, it is important to better define what gesture is. Gesture is the movement of any body part that occurs concurrently with speech ([189] $\mathrm{p}$ 23). Any body part movement performed without the presence of speech is not considered gesture.

For McNeill, gesture plays a very important role in thinking, and together with language, help constitute thought ([189] p 245). An interesting example is that of Gail's, as Crowder described [58]. During a science class, the girl used her closed fist as the sun shining on earth, represented by a globe, and said that the sun rays fall "straight on the equator". She then saw that her "sun" was not positioned straight over the equator, and she then adjusted her hand position and said: "No, right about here". Clearly her gestures had an impact on her 
words. Based on examples like Gali's, McNeill suggests that gesture is part of the process of utterance formation, and that "utterance formation is itself a continuous transformation of thought" ([189] p 29). Goldin-Meadow ([99] pp 180-181) affirms that "the act of gesturing promotes spatial thinking and not gesturing inhibits it", and continues: "Gesture doesn't only reflect the incipient ideas that a learner has, but actually helps the learner to formulate and therefore develop these new ideas."

So far, we have tried to show the importance of gesturing and how, together with speech, it shapes thought. However, at this point one very important question is: Can the congenitally blind gesture despite the fact that they have never seen someone gesturing? The answer is yes. Iverson et al [130] showed that "congenitally blind speakers gesture despite their lack of a visual model, even when they speak to a blind listener. Gestures therefore require neither a model nor an observant partner". In the same publication, Iverson observed that blind participants gestured at the same rate as those who can see. Goldin-Meadow 98 also found blind speakers gesture routinely even though they themselves have never seen gesture [98. Iverson [128] also found blind children gesturing when describing scaled down spatial layouts. McNeill states: "That the congenitally blind gesture at all as they speak is itself evidence of a speech-gesture bond". Lack of vision evidently does not impede thinking in gestural forms." ([190] p 26). We now move on to discuss the special role gesturing plays in mathematics.

\subsubsection{Gesturing and mathematical thought}

Roger Penrose, mathematician and physicist, wrote in his book, The Emperors New Mind, wrote: "almost all my mathematical thinking is done visually and in terms of nonverbal concepts, although the thoughts are quite often accompanied by inane and almost useless verbal commentary, such as 'that thing goes with that thing and that thing goes with that thing' " (212] p 424). McNeill ([189] pp 167-168) and Smith (248 pp 13-17) show that gestures can convey mathematics content and that it appears on all levels of knowledge, from professional to student.

When asked to describe his thought processes, Einstein wrote:

"The word or the language, as they are written or spoken, do not seem to play any role in my mechanism of thought. The physical entities which seem to serve as elements in thought are certain signs and more or less clear images which can voluntarily reproduced and combined." Hadamard ([108] pp 142-143)

Another interesting evidence is brought by McNeill [188. He videotaped discussions among mathematicians. He found that they had a "gestural vocabulary" representing some mathematical concepts. McNeill found gestures for: quotients, factoring, maps, flatness, and compactness/finiteness. Interestingly, the key features of those gestures remained throughout the utterances of one speaker and across speakers. This vocabulary was so strong that 
when one speaker uttered a concept and used the gesture corresponding to another concept, the listener corrected him based on the gesture. Normally, gestures are idiosyncratic as they are part of one's thought. In the case of those observed by McNeill, they function more like words, as the author himself pointed. It is like if no word could convey the idea better than the gesture with those key features. Maybe what makes gesture such a good tool for mathematics is the fact that " it constitutes a medium that can be used to create objects, to show spatial relationships, to exemplify actions.", as Kendon ([154] p 197) observed.

It seems that gesturing in mathematics is even more fundamental than in other human activities. We have already seen that those who are blind are equipped to reason with the help of gesturing. But how much is a SBVI missing due to the fact that she cannot attend to the teacher's gesturing? And to what extent can our proposal really help? We will try to answer these questions throughout this chapter. For now, we know that teacher / student dialog will take place around images that will be accessible to both (poster and raised line drawing). We also know that those who are blind can, among other things, understand: other person's vantage point [114], perspective [113], the information presented in maps [112], and metaphors depicted in pictures "without laborious explanations" ([155] p 7).

\subsection{Gesture, Social interaction, Teaching and Learning}

Mead ([193] p 47) proposes that gestures become meaningful when they arouse in the person "making them, the same response which they explicitly arouse in the other individuals". Chomsky [43] proposed the "language acquisition device", with which "all children are endowed". What Chomsky meant is that there must be something in our body that enables us to understand language, which is "inevitably full of rules that are constantly broken by daily utterances", Chomsky [43]. Rizzolatti and Arbib originally found that "the rostral part of ventral premotor cortex (area F5) contains neurons that discharge, both when monkey grasps or manipulates objects and when it observed the experimenter making similar action" [226]. They called these neurons, the mirror neurons. In later experiments, the researchers found the same mechanism in humans and "it includes Broca's area" [226]. Mirrors neurons nicely fill the gap predicted by Chomsky and explain the fact that we extract meaning from the other person's embodied behavior and not only by speech ([152] pp 131-141). Very frequently, a gesture can convey information not present in speech [98, 38]. The problem with the blind is that they cannot attend to (some of) the speaker's behavior and therefore might not understand what the interlocutor meant. Put in Clark's action ladder ([52], pp 147-148) terms, there might be a communication breakdown due to channel failure - the listener cannot attend to part of the speaker's behavior. However, it is important to note the SBVI is very attentive to sounds and can extract meaning from them. In the classroom, she can benefit from the variations in the teacher's tone of voice, hesitation and intonation.

We have tried to show that a person who is blind is perfectly capable of understanding and "doing" mathematics. We also strove to present evidence that the fact of not being fully 
aware of the teacher's behavior harms the student/teacher communication. Obviously, it is through this communication that information is transmitted and new knowledge is built on the student's side. The question now is how bad is such harm? To try to answer this question, we first turn to the work of Radford [219]. The author asked participants to watch previously videotaped mathematics classes. A group watched the classes with the volume turned all the way down, the second with no image and a third had access only to the notes the previous students made during the actual classes. Radford reports that the subjects had a very limited understanding on what was happening during those classes (in all three conditions). Beattie and Shovelton [17] found that when people could see the pointing gestures, aside from hearing the speech, there was an increase of approximately $10.8 \%$ in overall accuracy of response, compared with just hearing the speech.

Children and teachers derive information from each other's gestures, and this information can play a crucial role in the communication that happens in instructional interaction (99] pp 241-247). "Gesture can convey information that is not explicitly encoded in speech, thus providing a unique window into the mind of the speaker", Iverson [129]. For Kendon, "the speaker not only may provide information for recipients about his ideas, he may also provide information for himself about his ideas" ([154 p 81). This embodied awareness is mutual and the teacher can learn how to "read" and react to signals her student's body is giving away. Kelly et al [151] show that when an adult seconds or gesturally elaborates on a childs gestures, it may serve to reinforce the meaning of those gestures for the child. The authors report great improvement in student performance after teachers were taught the technique just described. This "sharing" of gesture features might mean that the conversants are sharing the same image associated the with the gesture, as pointed to by Kimbara [157]. Furyama [81] showed that learner can appropriate gestures of the tutor in a highly spatial task. Goldin-Meadow and Singer [100] found that children were more likely to repeat the strategies when these strategies appear in the teachers' gestures as well as in speech.

Goldin-Meadow found that when a child presents conflicting information in gesture and speech, this is a sign that she has, at that point, two competing thoughts on her mind and that indicates that she is in the edge of her competence ([99] pp 241-247), what Vygotsky calls "zone of proximal development" ([276] pp 84-91). The author also found that is in that stage that the child benefits the most during instruction. Kraut and colleagues [164] showed that in the absence of visual information, people tend to be more precise in their utterances, which demands more effort. How much more communicative energy should both the teacher and the SBVI put so that the student reaches the zone of proximal development? This extra effort has a cognitive toll and might partially explain why SBVI get tired more frequently than non-SBVI [252, 93. One of the objectives of this research is to observe what changes in the content uptake by the SBVI when such effort is lowered.

We presented evidence to give an idea of the instructional deficit caused by the fact of not being able to attend the teacher's gestures. Obviously, the intervention on the teacher/student interaction can only go that far. Only one type of gesture will be delivered to the student: Pointing. What kind of change can this bring? In the next section we will take a deeper 
look on how and when we point.

\subsection{Pointing}

According Kendon ([154] p 17), since ancient Rome, researchers have been proposing ways to classify gestures. The author compiles a comprehensive list of different classification schemes and concludes: "No attempt should be made to develop a single, unified classification scheme, since so many different dimensions of comparison are possible". McNeill, who also proposed a classification scheme ([189] pp 12-18), agrees with Kendon and affirms that the categories he proposed should be seen as dimensions ([190] p 41). McNeill also affirms that sometimes it is hard to assign a category to a gesture as it can fall into two categories or dimensions. One type of gesture appears in most classifications proposed, and recognizing it is not so hard: Pointing or deixis. We normally point using our hands, but it can also be done with the head, eyes, protruding the lips [245]. Our study, however, is confined to the pointing done by the hands. From this point on, whenever we use the term "pointing", we mean pointing with the hands.

There is the pointing at physically present object and there is the abstract pointing - when "space and a locus within it are used to present a nonspatial meaning", McNeill et al [192]. An example of abstract pointing would be during a discussion about moral, the speaker points to the right every time she is meaning that "that was the right thing to do", and conversely, points to the left whenever she is referring to something wrong.

It seems that the shape of hand plays a role in what we mean when we point. Kendon ([154] pp 205-222) presents a comparative study of hand shape used in pointing. From his collection of recordings (made in both Italy and England), he identified seven different types of manual pointing. They vary in terms of shape of the hand and arm movement. The differences in pointing emerge from the way the object being pointed is referred in the discourse. For instance, the object pointed to might be an example of something or illustrate a concept or be distinct from other objects.

Before we start discussing the several hand shapes, we must acknowledge that the $H D S$ is not capable of conveying neither the shape of the hand nor the movement of the arm to the SBVI. However, since gesture and speech are co-expressive ([189] p 23), the student might be able to "fill in the gap", via the information contained in both speech and the context. We shall now discuss the types of pointing.

Index Finger Extended (Palm down) This pointing, figure 1.1 a, is normally accompanied by deictic words like: "This", "That", and it singles out an object to which the speaker is calling attention. With the arm and index finger fully extended, one can say: "That is the Cathedral". 

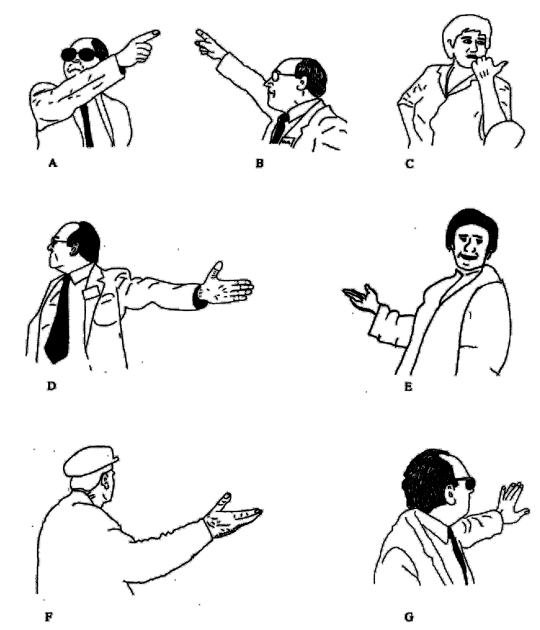

Figure 1.1: Seven different ways of pointing, from Kendon [154], page 206

Index Finger Extended Neutral (Palm vertical) Depicted in figure 1.1 b, and according to Kendon, this gesture is more used when we want to compare two objects that are sequentially pointed by the index finger, when we want to say something about that object - other than just pointing it, or if the object is a "cause or condition of something else". As when a person, seeing professional video markers working outdoors, points at a camera sitting on a tripod and ask: "In what television show will this appear?" Furthermore, this kind of deixis need not to be accompanied with deictic pronouns, nouns or noun phrases e.g., pointing at a map while saying "the town of Blacksburg is ...".

Pointing with the thumb In all Kendon's observations, we point with the thumb (figure 1.1 c) when the object being pointed at was "either to the side or to the rear of the speaker" ([154] p 218). This kind of pointing was observed when either the precise location of the object was established by a previous index finger extended, or when the conversants already know what the referent is. The object is no longer the focus of the discussion in any of Kendon's observations. In a classroom, the teacher could present a graph showing the demographic growth of a country in a time period, turn her back to the whiteboard, and facing the students make general comments about what was just explained, pointing at the graph with her thumb.

Open Hand Neutral (Palm vertical) Here the object being pointed at is either an exemplar of a class or "something that should be inspected in a certain way" ([154] p 212) (Figure1.1d). An example of that would be a person pointing at the speedometer and saying: "Please slow down, you're over the speed limit". The speaker is not actually referring to the 
speedometer. She is referring to the speed. A simpler example would be: "This is the type of car I'm looking for".

Despite the proposal of two different ways of positioning the hand when the index finger extended (Palm down and Palm vertical), Kendon acknowledges the fact that these differences were not consistent throughout his observations. However, he did observe interesting differences between the index finger extended (both palm positions) and the open hand pointing. According to the author, when the index finger is not used, the object being referred to is not the focus of the discussion, instead, the discussion is on something to which the object is linked (an instance of a class, or the location where some activity is happening). The use of deictic words is more frequent when pointing is done with the index finger extended.

Open Hand Supine (Palm up) We use such hand posture (figure 1.1 e) when we present an object that should be inspected in a "particular way", suggests Kendon ([154] p 212). Suppose a dialog between a person shopping for a horse and a seller. The seller would say: "Look how strong this horse is.". This utterance could be accompanied by the seller's hand being raised, his arm extended and his hand opened with its palm turned up. Just like index finger extended neutral (palm vertical), this kind of gesture need not to be accompanied with deictic pronouns, nouns or noun phrases.

Open Hand Oblique Kendon observed that in such style of pointing (figure 1.1 f), the object being pointed at is a person and the comment towards that person is negative. As when in a team discussing the reasons they just lost a match, "I think it was John's fault", the speaker directs his hand opened and oblique to John.

Open Hand Prone (Palm away) For Kendon, this type of pointing (figure 1.1 g) normally follows the Index Finger Extended (Palm down) gesture. It is normally used to draw the listener's attention to some specific feature of the pointed object in relation to others. Kendon's example is that of a tourist guide showing buildings with different architecture styles but in the same neighborhood. The guide first points at the buildings in general, then points to a particular one (both pointings with index finger extended), and finally presents a particular feature on that singled out building which the tourists would find interesting knowing. In the described scenario, the guide would say: "Look at this building, see how it has the same style of the others, even though it was built in a different era."

The discussion of the different types of pointing alerts us to the multiplicity of scenarios in which the blind could have her understanding of what the teacher is meaning enhanced by the proposed system. Nevertheless, it is important to note that Kendon's analysis were performed over data collected from England and Italy. Kendon acknowledges the important role culture plays in gesturing. However, these are two western cultures. Do people in other cultures point differently? We try to answer this question in the following section. 


\subsubsection{Gesturing, environment and culture}

Italians are known to gesture a lot. Some say it is due to their crowed eighteenth century cities. In those times, people used gestures to communicate in an environment where many people were talking loud and simultaneously. So, people gestured to both help communicating in a noisy environment and to compete to other people's attention (63] pp 102-103). Efrom [71] showed that the American decedents of both Italian and Jewish immigrants gesture alike despite of the great disparity of their parents gesturing. The studies above mention gesture in a very broad way, one might ask: Do culture and environment play any role in pointing? According Wilkins [293], the answer is yes. The author presents his observations among the Australian aboriginal group called Arrernta. Those people point with both index and little finger extended, and the two middle fingers flexed downwards when they want to indicate route or direction. They also point with all fingers spread and palm facing downwards to indicate multiplicity of objects. Kendon [153] also studied tribes in Australia: the Warlpiri and the Warumungu. The members of these tribes raise their arm as a function of distance the object being pointed. The further the object is, the higher the arm is raised. However, when pointing was used to single out an object, the extended index finger posture is adopted across the cultures examined.

Another interesting aspect is that gestures tend to be idiosyncratic as they emerge from each individual's form of expression ([189] p 248). However, for Kendon, "the pointing gesture is not a matter of idiosyncratic choice or variation unrelated to the other things the speaker is doing. It seems, rather that the hand configuration a speaker uses in pointing is a patterned component of the utterance ensemble. It is as if the speaker uses a different form for the pointing gesture according to how the object being referred to is used in the discourse." ([154] p 225).

We briefly looked on how issues like culture and environment can give different meaning to a simple gesture like pointing. We also revealed that our system is not able to deliver important aspects of the pointing like hand shape and arm and path both arm and hand take during the gesture. We shall move our discussion to a scenario that we imagine plausible on pointing with the help of the HDS.

\subsubsection{Pointing combined with description}

We borrow from Kendon ([154 p 202), this interesting example of a woman at a fruit stall (figure 1.2a). The lady points at the scale and complains about the scales: "They [the scales] go down, they are not going down! Cheat!". As she utters these words, she points at the scales and rotates her arm. The pointing is to establish that she is referring to the scales. She then makes the movement corresponding to what the needle does. Kendon posits that lady is building a body-model ([154] p 202) of the needle. The movement performed by her arm is the movement she sees the needle doing. These hand movements can be seen as two 
distinct gestures. The pointing is followed by a modeling.

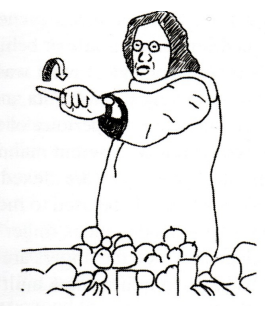

(a) Pointing combined with description, from Kendon [154], page 202

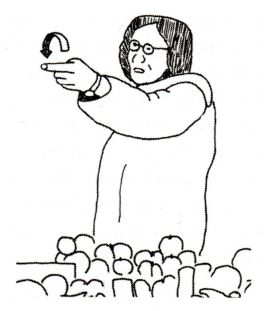

(b) $2 \mathrm{D}$ representation of a scale

Figure 1.2: Pointing and description

One thing on the example above catches our attention: the needle model is conveyed in two dimensions. The lady only swings her finger from left to right or vice-versa. A twodimensional model of the needle can be easily printed to a swell paper (Figure1.2).

McNeill shows that thoughts are dynamic combinations of image and word ([189] p 220). We must acknowledge, however, that images are not necessarily created in our mind in two dimensions. We need them precisely because thought is multidimensional as opposed to the linearity of the discourse (99] p 25). Goldin-Meadow (99] pp 25-26) gives a nice example of a multi-dimensional gesture: A horrified boy describing how he was chased by a spider. The boy's hand becomes the spider and his fingers, the spider's legs. Then, his hand starts to move like the spider, following the same path the spider took. The speaker clearly uses the three dimensional space to convey his thought.

On the other hand, the whiteboard/blackboard is one of the most efficient tools available for teachers. Every day literally millions of teachers draw and write on them. Furthermore, they rely on these illustrations to lecture. So, one might ask: What is the relationship between the drawing and that image described by McNeill? They probably share some key features, the indispensable ones to convey a certain meaning. This sharing would partially explain why drawings are largely used in lectures and presentations. Returning to the scales figure, only by looking at the drawing, one cannot understand the swing of the needle. The concept is fully conveyed when the teacher drifts her finger over the figure and simultaneously explains why the needle swings - what McNeill called the dynamic combination of image and word. We posit that our system would increase the chances of understanding by the SBVI. This is so because the student's hand would be directed, by the system, to follow this drifting move. Now, we return to the mirror neuron mechanism. Rizzolatti and Arbib showed that doing and observing someone else doing the same action, activate the same brain area [226]. In other words, if the student is led to repeat the teacher's hand movement, such movement should activate the same brain area as if he were seeing the teacher. Furthermore, Harber [107] has shown that people who are blind can understand images rendered in raised-line drawings. So, depending on the teacher's strategy and use of this $H D S$, the SBVI might get 
more than just the understanding of the teacher's deictic gestures.

The discussion above was on a how far could teaching go with the help of the HDS. We arrived here after a review on gesture, language, thought and how they combine. After that, we showed that a teaching strategy might have a crucial role in helping the student in the construction of his own knowledge. However, those were only hypothetical scenarios. We shall discuss real teaching practices and learn from their experiences.

\subsection{Teaching the blind}

There is a school for the blind and visually impaired in every State in the US. Among those schools, Texas School for the blind and visually impaired (TSBVI) [77] is one of the most vibrant. On the TSBVI's website, there is a collection of pages on how to teach mathematics for those with this special need. However, the strategy of segregating the disable has been under heavy criticism. Inclusive classrooms, where people with disabilities attend the same class as those who do not have them, have been the educators' preferred choice for some time. Inclusive classrooms are beneficial for both disabled [64] and non disabled students [254]. They are required by law (Individuals with Disabilities Education Act Amendments (IDEA, 1997), and the No Child Left Behind Act (NCLB, 2001). Court ruling [263] had also reinforced the non-seggregational approach. Therefore, the discussion is not if inclusive classrooms are good or not, it is how to make them work. The reduction of the gap between special and regular students requires both inclusion of those with special-needs and effective educational methods for all students [15]. One of the most promising practices for helping students with disabilities succeed in the classroom is the use of technology [14]. Our proposal is consistent with inclusive classrooms because there is no requirement for the teacher to change either her behavior nor her class notes to use the HDS. The only action required is the production of a raised line version of the notes offered to those students who can see. We shall discuss the strategies developed in previous cases, the role of the tutors, the instructional materials used and debate on the authors' recommendations.

We shall begin discussing how McCalistar [182] tutored Edward, a congenitally blind, was his undergraduate statistics course. The tutor recorded a book at the beginning of the semester. Edward had a compact audio taped version of the classes which he used to study for the exams. He also had a talking calculator, but he found easier and faster to instruct someone to do the calculations for him. Unfortunately, Edward did not use raised line drawings because the university had not installed the equipment at that time. Edward had extensive tutoring. After the lectures, the tutor had to go over the concepts several times before attempting any exercise or homework. When under pressure, Edward skipped classes and relied entirely on his tutor. The fact of not having any visual experience only made learning harder for Edward. For instance, he had a hard time understanding the concept of pie-chart because he had never seen a pie. Statistics teachers like to use cards in a deck to explain probability because they think the students can easily grasp the concept of chance - not Edward - he 
had never seen a card. Another problem was with the greek letters that are present in many formulas - there are no Braille correspondents for them. Whenever possible, concepts were explained by body movements, what McCalister called "kinetic explanations". For example, the standard deviation was explained by stretching his fingers to the width of a standard deviation. The concept of the standard deviation as a descriptor for the "spread" was easily understood as his fingers spread. It is interesting to observe this natural surge of McCalister's kinetic explanations. How does this phenomenon relate to the extensive use of gestures in mathematics that McNeill found? Is this McNeill's dynamic combination of image and word, when again they were using the body to produce the image? Would McCalistar be forced to create this strategy if they had the raised line drawings available? Or if they had the raised line drawing, would the kinetic explanation, or more specifically, the gesture form, bare any resemblance with the image? Obviously, we will never know from this research. But the questions raise another interesting one: Given that a raised line drawing and a gesture may be used to convey the same concept, should they share the same key features? Our research might shed some light into this question. Although, we have not sought to answer this particular question, our approach is to carefully observe how both teacher and student will convey their ideas both during and after instruction. We will videotape all the instruction throughout the mini-course we are proposing. We will also videotape the blind students when they are attending the lecture and when they are taking their oral exam. All the videos will be carefully analyzed and coded. Later in this document, we detail on how classes, oral exams and coding the videos are to be conducted.

Returning to McCalister's experience, he also found Edward getting tired fast and frequently. To combat that, McCalister decided to use examples with a very small data set and one memorable problem for each formula studied. It seemed to work, but as we will see below the fatigue problem seems to be recurrent among the cases studied.

Spindler [252] assisted Peter during two semesters of calculus. Peter still had vision when he finished high school. The author reports that this visual experience was very important and helped him communicate concepts verbally to Peter. Here we find a sharp difference from teaching a congenitally blind and a late blind. In the first case, a simple example referring to a deck of cards can be an issue. This shows that the challenges of educating those with this condition are far greater than this research's objectives. However, we will accept help from those who are blind with no distinctions on what stage of life they lost their sight. This is mainly due to the difficulty of finding willing subjects. We will return to this issue later.

Returning to Spindler and Peter, several teachers used powerpoint presentations and with that many deictic expressions like "this" and "that" were uttered during lecture. Obviously, Peter had a hard time catching up with the explanation. Spindler also found mental fatigue. Several times the tutoring had to stop because Peter got tired. That normally happened when he was doing homework problems that demanded calculations to be performed in a step by step basis. In those situations, Spindler became Peter's "paper" and wrote down whatever Peter asked. Whenever necessary, Peter would request Spindler to read back to him the information from the paper. What Peter was actually doing is cognitive offloading 
onto the environment [296] with the Spindler's help. Spindler found the use of raised line drawing quite useful when they covered two and three dimensional Calculus. He used sheets of paper in various forms to help Peter "visualize" surfaces in space. Still, problems involving multiple dimensions were always hard for Peter because he usually had to hold too much information in his head to solve the problems.

Gibson [93] also discusses his experience in teaching statistics to a blind student. He advocates the use of tactile pictures, following Heller's advice ([115] pp. 239-261). These are obviously more indications that the raised line documents approach was largely and successfully used in previous experiences. Gibson also explored what he called "manipulatives": Cardboard and modeling clay. The idea is that the student could, through touch, explore the contents of these haptic displays. The normal distribution, for example, was portrayed using modeling clay. The cardboard was used to convey bar graphs and "bars of different heights depicted means for a control group and an experimental group", for example. This is a common technique in schools for the blind. One problem is bringing these strategies to inclusive classrooms. Later in this document we will address some haptic devices suitable for this kind of instruction. We anticipate that they are expensive and difficult to build.

Another interesting case is that of Cary Supalo's [257]. In this work, he describes his own experiences as a blind student pursuing his doctorate in chemistry at Purdue University. Supalo described which learning strategies worked and which did not. The author affirms that he has consulted with other students who are blind on the strategies and devices used. For Supalo, it always better for a blind student to sit in the front row so that she can pay more attention to the instructor and not get disturbed by sounds other students make. Some students bring a sighted person to take notes for them. The problem is that notes tend be idiosyncratic [181]. Furthermore, they need to be transformed into a format that the blind can read and understand and this takes time. Despite being "tedious and time-consuming", this was one of Supalo's preferred strategies. There are Braille based electronic note-taking devices that some students take to class. Their problem is that they are text-based and are very limited for use in mathematical and chemical expressions. An alternative, according to Supalo, would be the use of a slate and stylus. They are cheap and can be used directly on a braille document. The problem is the noise they make when the user perforates the paper to make her notes readable. Some students also bring tape recording devices to class. Here, the problem is in listening to the tapes later. It takes roughly the same amount of time the lecture took. Supalo recommends the use of tactile figures. The instructors gave him raised line versions of the transparencies they would use during the lecture. There were special labels on Supalo's documents. Verbal references to the labels served as cues to where Supalo needed to move his hand to stay grounded to the discussion. In all cases discussed above, the task of teaching rely heavily on the tutor's side. Even Supalo acknowledged the indispensable help of his sighted note-taker. This role is also prominent in schools for the blind, like the one in Texas. We have seen in cases like Edward's that the tutor practically taught him the course. The tutor attended the classes and regurgitated the lectures. We reach the conclusion that although inclusive classrooms are preferable, they are far from the 
economic feasibility. It is just too expensive to have a one to one tutoring. This shows that there is a wide open avenue for research and development in the area. Furthermore, as we pointed before, this population (individuals who are blind) offers an unique perspective to study the processes involved in learning.

Other recurrent issues are fatigue and the need for note-taking. Maybe they are related. Attending class might pose such a heavy cognitive load on those students that they get tired earlier and desperately need to offload it (via notes). It seems so important that the students went to great lengths to have their notes: They hire a person just to take them during class and have them translated afterwords. We see this as a research opportunity.

Such load might partially come from the impossibility to simultaneously attend to the teacher's discourse and the instructional material. The student puts too much effort in trying to "fill in the dots" (eg. understanding deictic expressions). The recommendation of sitting in the first row can be seen as an effort to obtain audio cues that could increase their understanding of the teacher. More than that, Supalo's special labels are a striking example of the need stay grounded on the teacher's discourse. This is one of the core objectives of our research. If the student can understand the teacher with less effort she will probably get less tired and even participate more actively in class discussions. We know that students who are more engaged in classrooms activities perform better [30, 36, 80]. This view is shared by Bardin ([16] p. 1) who affirms that it is very difficult for those who cannot access visual information and nonverbal communication to participate more actively in classroom discussions. At this point we can raise several questions. How much will our intervention increase the student's awareness of the teacher's embodied behavior? Will this lead to more engagement in classroom activities? and to better performance in the oral exams? Will those who attend instruction with the help of such system be able to express the concepts they were taught more precisely and less effort-fully than those who are also blind and attended to the same lecture but without the help of such system?

This discussion on the techniques used to teach the blind, among others, showed that because of the lack of vision, tutors were more prone to explore other sensorial experiences to convey concepts. Some of them are not useful in inclusive classrooms, while others would depend the construction of complex and expensive equipment. In our proposal, the haptic exploration over a raised line document remains. Such exploration is expected to be performed as the teacher explains the concepts. How much is this motor activity beneficial to the process of learning? One thing we know: The students will be engaged in a richer multi-modal activity. In the next section, we try to relate sensorial with cognitive activities.

\subsection{Embodiment Awareness and Cognition}

For Quek [218], Embodiment awareness is "the ability of the recipients of communications to take in the full range of multimodal elements of the communication ". Key to our re- 
search is providing the SBVI with a richer learning experience by increasing their chances of understanding their teachers. How does Embodied Awareness relate to Cognition? Let us suppose for a minute that we can raise the student's embodiment awareness of the teacher. Will this make her a better mathematics student? Well, her perception would definitely be raised, but how would that effect her reasoning and judgement?

For Lakoff and Johnson [168, "meanings are developed through embodied experiences, both in relation to the self and to others". For Stinson [256], educators should "cultivate kinesthetic sense and use kinesthetically rich images in our scholarship to help us and others perceive more clearly, understand more deeply, the embodied others who are subjects if not participants in educational research". To what extent does the tactile exploration of the raised line drawings resemble the use of the strategies proposed by Stinson? In the last section, we have seen isolated cases of tutor using certain body movements to help their blind pupils to grasp mathematics concepts. We shall continue the discussion on body and mind.

When Lakoff [167] claims the mind is "embodied", he is arguing that almost all human cognition, up through the most abstract reasoning, depends on and makes use of such concrete and "low-level" facilities as the sensorimotor system and the emotions. Tall [258] gives a nice example of how abstract thinking is rooted on bodily experiences. For that, he introduces two concepts: Set-before and met-before. The first one is about the brain abilities that everyone has since birth. A baby, for example, has a natural ability to count, to distinguish form shapes, to track moving objects, etc. We build new ideas based on contexts we meet, and on mental structure we have then. Thus, a met-before is a previously constructed idea that we use when addressing a current situation. Following the author's reasoning, we can start teaching mathematics to student using his set-befores. In subtraction, for example, we can use the "take-away" set-before. Obviously, this does not work if the result is less than zero because you cannot take away more than you have. Such situation triggers the need for introducing a new concept: negative numbers. Tall explains the importance of embodied experiences in mathematics teaching. When teaching division, the instructor can actually divide a physical object. After that, we can "compress" the knowledge using the corresponding symbol ":" and embody it. Once embodied, that concept can be used as a building block to other concept, which can be more distant from a body experience. As we advance in the study of mathematics, we rely more and more on symbols to compress concepts. The next natural step in the cognitive growth is the association of concepts to produce new ones.

In his book, The Body in the mind The Bodily Basis of Meaning, Imagination and Reason, Johnson [147] explains the term Image Schema, as a structure frequently used in our cognitive processes, which establishes patterns of understanding and reasoning. Image schemas are formed from our bodily interactions, linguistic experiences, and historical context. They are multi-modal patterns of experience, not simply visual. There is no reason to doubt that those who are blind use image schemas in their cognitive processes. We imagine that our system will provide richer multi-modal experience which might help the students in the creation of such schemas. 
Another way to frame the argument that this richer multimodal experience might lead to better student performance is through activity theory. The traditional notion of mental processes in cognitive science is termed internal activities in activity theory. According its followers, internal and external activities cannot be understood separately since there are mutual transformations between these two kinds of activities. Internalization is the transformation of external activities into internal ones. "Activity theory argues that it is not just mental representations that get placed in someone's head; it is the holistic activity including motor activity and the use of artifacts that are crucial for internalization.", Nardi [201]. Nardi gives an example of a child learning arithmetic. She will start using her fingers and once the arithmetic is "internalized". She can do the calculations only using her head.

Externalization is important because it enables collaboration among several people whose activities need to be coordinated. This is what a teacher does in a classroom; she externalizes the concepts so that her students can internalize them. This "lesson activity" is therefore mediated by the tools the teacher and students have at their disposal. Examples of such tools are: the language itself (including gestures), the blackboard, books, etc. For the activity theorists, tools shape the way human beings interact with reality. One can expect that by shaping the tools we would be forcing a change in externalization. Furthermore, according to the internalization/externalization principle, the shaping of external activities shapes the internal ones.

We return to the question how much can the instruction can benefit from our new tool. It seems that it will all depend on how teacher/student will use it. Teachers who are aware of the benefits of the haptic/kinestic exploration in might adopt strategies that would make more use such resource as they lecture.

\subsection{Transition}

In this initial chapter, we laid out the foundations of our research. We first explain, in a very high level, what are the motivations of our research, what we intend to do about them, how we plan to do and how we will assess the changes our intervention will bring to teaching those who are blind. After that, we tried to explain the deep connection between language, gesture and thought. There we strove to make clear that gesture and other embodied behaviors are part of our thinking and self-expression processes and that the blind are endowed with the same capabilities despite the fact that they have never seen their own gestures nor anybody else's. After that, we show that gesturing is particularly important in mathematics, being part of the "practitioner's vocabulary". Following on that, we explain that because the blind cannot attend to the teacher's gestures, they miss an important part of the meaning that is being conveyed. The next step was to show that we do not have the intention nor capability to convey all the embodied cues the teacher ever gives during a lecture. Rather, our objective is to convey the teacher's pointing. 
Pointing, in turn, is a very powerful linguistic tool. We laid how differences in hand shape and arm path during the pointing may lead to differences in meaning and, at the same time, we acknowledged our incapacity to convey such nuances, leaving the final interpretation to the student. We also showed that culture and environment are always strong factors in shaping the meaning of our speech and gestures. Even a simple gesture like pointing might convey different meanings among different cultures. Our response to that fact is that, again, the final understanding of the gesture is given by the students, who are probably from the same culture as their teachers. We moved on to posit that in most cases, the teacher will point to a figure as she describes it. This pointing combined with description might lead to a deeper understanding of the concept if the student follows the contours of the figure as the teacher describes it. It is plausible to believe that the student might understand the body model associated with the concept. This will depend on the teaching strategies that will arise from the exploration of the new communicational capabilities our system offers.

After the discussion on pointing we examined previous experiences on teaching the blind. We learned that there is a need for technology that enables inclusive classrooms. Unfortunately, we also showed there is still a long way to go for that to become feasible. The good news is that projects like ours can help to promote inclusive classrooms. The discussion also showed us the need blind students have for a note-taking tool. This need might be a sign that the cognitive load on those students is too heavy, and for that we presented more evidence. It might be because the student puts to much effort on trying to "fill in" the communicational blanks, as she cannot attend to the teacher's embodied behavior. The strategies employed by the tutors were highly multi-modal as explicated by our last discussion on how a richer sensorial experience can effect cognition.

We are now ready to move to the next chapter which will address perception. There, we will posit that humans use the combination of multimodal sensory to help in disambiguation - a crucial process in making sense of the world. The discussion will narrow down to a comparison between vision and haptics. We then move on to explore in more detail the haptic sense. After that we show how we built, tested and evolved our haptic glove. 

Chapter 2

On to Perception 



\subsection{On Perception}

Daniel Kish [78], who lost his sight as an infant, has taught himself to ride a bicycle. He now teaches people who are blind "how to see" and those who have sight how to "see better". Such a feat can only be accomplished through the gathering of multi-sensorial information. He needs equilibrioception, the sense of balance, not to fall from his bike. He also needs proprioception to coordinate his arms and legs movements. Furthermore, he needs information about the terrain he rides on. Different terrains require different skills. He needs to pedal harder when going uphill. The bicycle demands different body attitudes from its rider on asphalt, grass, gravel, or mud. Finally, there is the navigation. Kish learned how to use echolocation. Similar to a bat, he emits click (with his mouth and tongue) and pays close attention to how they bounce back. Slight differences give away obstacles, and other distal objects.

Kish's skill is a good example to start our discussion on perception. Similar to Kish's clicks, "our eyes do not record the environment. They interrogate it.", Yarbus [74, 299]. For Kennedy ([155] p 2), "perceptual systems evolved as detectors of useful properties of surroundings". These "useful properties" are what distinguishes Sensory Processing from Perception, according to the followers of the Human Information Processing Model - HIP [291]. During his ride, Kish can feel the cold wind on his face, but that sensation might not be useful for the task of riding the bicycle. On the other hand, if the click indicates a barrier, he will change his course.

The bike riding example shows the use of the information gathered from a multiplicity of sensory channels to accomplish a complex task. We use the information we gather from the environment to make sense of it. This multimodal sensory information helps us disambiguating uncertain information, Oviatt [208. The wind hitting his face stronger and stronger, his center of gravity a bit shifted forward, the lack of effort, might, combined, mean that Kish is going down hill. If he were able to perceive just only the wind blowing harder, he could think that it was just a breeze. The multiple sensory information is necessary from disambiguation. Depending on the task, the interaction with the environment demands rapid responses. If Kish fails to give meaning to his sensory experience and not respond in a timely fashion, he will fall from the bicycle. Perhaps, one of the reasons for Kish's success is the vividness of the sensations the experience yields. The bicycle makes pretty clear what "it wants". Losing balance demands more pedaling. Going down hill, braking. Rich sensory evidence is key. Wickens ([291] p 12) citing Rumelhart [230] posits that "when sensory evidence is poor, perception will be driven heavily by our expectations based on past experience". Past experience and long term memory are hallmarks of cognition, not perception, Wickens and Hollands ([291] p 12). There is no room for too much thinking for acting when it comes to staying on top of a bicycle.

How does Kish's experience relate to a blind student attending to a class lecture, besides

the fact that in both cases they are blind? Kish needs information from several senses to 
understand his bike's behavior. A blind student also employs all sensory channels available to her to understand the teacher's behavior. Definitely, many of the teacher's intentions are perceived through his speech. In speech, not only words convey meaning. Prosodic information like pauses and different pitches also do. Furthermore, through subtle sound differences, it is possible to know when the person is speaking towards you or when she turns to the side, to use the whiteboard for example. In the classroom, the student is engaged in this social interaction, which includes other students and the teacher. She can also infer meaning from the behavior of her colleagues. Questions and comments made by others play an important role in raising the blind student general understanding of the lecture. However, what seems to be key is the ensemble of perceptual information. One missing piece might prevent a person from performing well in a given activity or ruin her performance on other. In Kish's case, he has learned to perceive at distance using his clicks. He could not ride his bicycle if he had not first developed this unique skill. What Kish did was a sensory replacement - hearing replacing vision. This is precisely what our approach seeks to enable, by providing a sensory substitute for embodiment or deictic awareness. In our approach, haptic exploration substitutes sight. We argue that, by enabling a richer multimodal experience, the SBVI will better understand what is happening in the classroom.

Before we go any deeper into this discussion, we need to differentiate haptic from tactile. Haptic can be divided into tactile and kinesthetic information. Heller and Schiff [115] help us to draw to distinction. Tactile is related to what is perceived by cutaneous sense, such as: Temperature, texture, pain, and vibrations. We receive kinesthetic information through our bodily position, weight, movement of the muscles, tendons, and joints. When a SBVI uses the glove, she is truly using haptic perception (the combination of tactile and kinesthetic) to navigate over her class notes. She perceives the information through the vibration the motors cause on the palm of the hand (tactile). This information is related to his/her hand position (kinesthetic).

Having clarified this distiction, we now return to our discussion on perception. Kennedy ([155] p 8) sees perception as a "set of vantage points and therefore needs time to collect variations". Gibson (92] p 84) posits that "visual perception is not an all-at-once photographic process of image-taking from the retina to the brain but a process of exploration in time". Vision and touch use the same tactics to observe the geometry of surfaces which can be flat or curved ([155] p 9). In vision, Kennedy ([155] p 3) argues, variations of colors and contrasts give us clues to identify objects by delineating their edges. Touchers also employ the "edge strategy" to identify objects [91]. Edges appear in line drawings as they do in vision ([155] p 6). For Bach-y-Rita ([11 p 45), edges seem to convey the "most important information of pattern recognition". The obvious disadvantage for touchers is that they can only "see" few parts (at a time) of an object and therefore they need time to unify the perceived parts ([18] p 375). When they do not have time, they cannot organize correctly the parts of the object.

Perceptual stimuli are related to motion through a medium ([155] p 12). Vision needs light waves. Hearing needs sound waves. Touch also needs motion and a medium. This normally happens when one of the body parts of the tactile system is in contact with a surface. This 
is one of the motives why touch is often seen as a "proximal perceptual system", one that can be used to interact with objects close to the individual. However, depending on the type of medium, touch can receive distal stimuli as well. Kish can perceive much useful information about the terrain through his bicycle's handle bars. Such stimuli are considered distal because none of his body parts are in contact with the origin of the stimuli. A person in the water can also feel the vibration of a passing swimmer. From the both examples, we might argue that those who are blind are familiar to distal haptic perception. A bit different but in the same realm, those who are blind understand tactile figures when those portray distant objects, such as maps and blueprints ([155] pp 45-55).

Touch can be either passive (when a person is touched) or active (when is the person who does the tactile exploration). Humans use both tactile perception modes (active and passive) simultaneously to perceive and act upon the environment [260]. This simultaneity is key for our research once the students both receives directional signals through the haptic glove (passive) and explore the content of the raised line drawings (active). Gibson observed a higher object recognition rate through active when compared to passive touch [91]. When such active exploration is guided by a tutor, the probability of recognition of a tactile picture is greater than when the exploration is unaided [178]. Haptic exploration is fruit of the collaboration between kinesthetic and touch. The exploration of tactile figures is normally done with one finger [120]. Loomis et all [174] observed that the use of multiple fingers brings very little improvement to the exploration of raised line figures. They are useful when the exploring three dimensional objects [159]. However, Splinder [252] showed that is particularly hard to convey multi-dimensional concepts for the blind. Besides, building haptic devices to convey multi-dimensional information is very hard [134].

D'Angiulli et all [62] showed that congenitally blind and blindfolded sighted individuals have similar performance when recognizing tactile images, if they are properly guided. This is evidence that visual experience does not to play a role on such process. It also conforms with the fact that images are formed in the brain [127, 137]. In addition, it has been proven that congenitally blind adults process spatial images in a similar way to those who can see [137, 161]. However, this processing requires slightly less time among the sighted [156]. Moreover, we are replacing a highly parallel sense (vision) [110, with a sequential one (touch) [91, 18]. On top of that skin has a much poorer resolution when compared to the retina 239. These challenges to SBVI result in the phenomenon that that teaching the blind takes longer than teaching the sighted [64]. Apart from that, we believe that SBVI are perfectly equipped to learn mathematics. The difference lays on perceptual strategies both groups employ. We shall now address some of these strategies.

\subsection{Uses of tact to acquire useful information}

Braille readers drift their fingers over raised dots arranged in such way to form the letters of the alphabet. They, however, read at one third of the speed sighted students do ([235] p 
135), [84]. This serves as a simple and trustworthy basis of comparison of the transfer rate of useful information via both senses (visual and tactile), as opposed to measuring information flow at receptor level ( [236] p 69), [160]. These numbers also encompass reading strategies senses afford [223, 166, 117]. It is not our intention to investigate in detail strategies the blind use to acquire information in every particular task. We want to understand how haptic devices can convey information and at what rate. Earlier, we tried to make the case that there is nothing we can do about the physiological differences between the two senses. So, what is left for us is to investigate ways of maximizing the acquisition of information via the haptic sense, and at the same time, making it cheap in terms of use of cognitive resources.

It has been almost half a century since Gerald [86] placed the first electrodes in contact with human skin. An exhaustive review of this body of knowledge is also not our objective. Instead, we will take "snapshots" of the history of the field and discuss the trends, both past and present. At the end of this section, we frame our research according to today's common sense.

In the late sixties, Bach-y-Rita and colleagues [13] developed an apparatus which had a twodimensional matrix of tactile stimulators capable of conveying previously scanned images. Only highly trained users could recognize the simple figures that were presented to them, on a single task. On top of that, such recognition normally would take from 5 to 20 seconds after the stimulus onset. We identify two problems with this approach. First is that the user does not have any control over rate she is receiving the stimulus. If she misses any bit of information, she will probably miss the whole message. We have already seen the users need time to put the "pieces" together. This might be the reason only well-trained users can ever recognize anything. The second problem is more of a consequence of the first. Such response time is likely to ruin any mathematics lecture. By the time the student had recognized the figure, she may have totally forgotten the context in which it is inserted.

Instead of conveying existing languages through skin (eg images of letters from the latin alphabet), some researchers created a new language to be conveyed through tactile stimuli, the vibratese [86, 244, 116]. Again, no more the $60 \mathrm{wpm}$ could be conveyed through skin [83. This result is worse than reading braille directly from paper. Add to that, the need to learn a new language.

The studies discussed above showed that it seems to be in vain to convey complex messages through the skin. Up to this day no haptic device could yield better results, at least in reading, than braille on a paper. We must acknowledge however, that most of the today's "blind reading" is done with the help of computers running with text to speech software (eg. Jaws from Freedom Scientific[238]). We can draw several lessons from these research efforts. The first, observed by Gallace et al [83, is that it seems that deciphering complex tactile signals is a heavy task, in cognitive terms. At this point, it is important to notice that in the research described above, the task involved simple actions, like recognizing letters (and words) or shapes. Student's attention must be primarily directed to the teacher. There is evidence that when haptic stimulus is presented in a dual-task scenario - with other 
concurrent information is being conveying in a different mode, the result is a dramatical decrease in task performance [251, 250, 172].

Second, still according to Gallace and colleagues, is that the current trend (as of 2007) is to adopt simple signaling patterns [83, 9]. Bach-y-Rita and colleagues [11, 12] adhered to this tendency and their devices emit only vertical, horizontal, and diagonal cues. Tactons [27, 31] operate as a notification aid to alert when an e-mail has arrived, or to serve as a substitute for a progress bar [28]- all simple and short signals. It seems to permeate all scenarios investigated. The strategy was also employed in tactile displays used to give directional cues to pilots who get disoriented when submitted to high gravitational forces [231, 269], to astronauts in orbit [266], to improve perception and composition of music [106], mobile phone (feel who's talking) [33, 32, even in the treatment of phobias [37].

So far we have seen that although there are over 10,000 parallel channels (receptors) in the human skin [56], using them to convey complex signaling seems not feasible. It gets worse in a multitask environment, so signals need to be short and simple. We shall now discuss the use of tactile stimuli in settings closer to ours.

\subsubsection{Haptics in math teaching for the blind}

So far we have seen that haptic displays have been successfully employed in a diversity of scenarios. We shall now narrow down our discussion to the research efforts that used the haptic sense to teaching/learning of mathematics. In this section, we start summarizing some of the previous research efforts related to our proposal. Only after that we will try to show where our project is different and why the difference is crucial.

McGookin and Brewster [187] discuss the work they had done on the MultVis - a project to allow visually impaired users to "both construct and browse mathematical graphs effectively". They developed a software, the graph builder, that works in conjunction with SensAble technologies' PHANTOM haptic device [242]. PHANTOM is a force feedback device that makes it possible for users to touch and manipulate virtual objects. The graph builder programs the PHANTOM so that the student who is blind can explore graphs through kinesthetic interaction. The authors, however, were not encouraged by the study results: "..it appears they (the subjects who are blind) do not perceive the same benefit in using graphs as would a sighted person".

Talking Tactile Tablet - T3 [283] is basically a tablet on which a raised line document can be placed. The users can explore the graph pretty much the same way they do with the raised line paper alone. The difference is that there is an extra possibility of interaction: the user can press the graphic and depending on where he presses, he will hear a pre-recorded explanation of that area of the graph.

VTPlayer 249] mouse has a collection of pins that raise according to the values of the pixels directly surrounding the mouse pointer. Wall and Brewster [279, 280] made a comparative 
study involving VTPlayer, the Talking Tactile Table (T3) and PHANTOM. The authors reported that participants that used T3 to explore graphs had the best performance. They were followed by those who used PHANTOM. VTPlayer users confounded the mouse with refreshable braille displays. They did not like the display size and its low resolution made almost impossible to understand the image they were investigating [280. Jansson and Pedersen also found blind subjects having difficulties with the haptic mouse [135]. Manshad and Manshad [180] also studied haptic aided graph exploration.

Where does our approach differ from the above mentioned and why we think our proposal covers different and important aspects the former approaches neglected? First, we formulate the problem as that of situated discourse, whiled prior work focus on the act of reading or acquiring information. In none of the afore discussed works we see a real person guiding the apprentice. This is crucial to learning. The student should be able to stop and ask questions, and according the answers she receives, to revisit portions of the graph to clear a misunderstanding. Only then should the teacher proceed with the explanation. Winberg and Bowers [297] found that in a collaborative task involving blind and sighted people, a shared representation is important. Luhse [173] observed that different graph representations can have a large impact on time and effort to extract information, even if they represent the same information. This phenomenon might be due to the need of both conversants to have the whole graph schema in their short-term memory during their work [214]. This would force them to make complex mental transformations from between both representations [300]. Mynatt and Weber point that cooperation is assured when coherent visual and nonvisual interfaces are available [199]. One of the strengths of our approach is that both teacher and student have the information represented in essentially the same format and this prevents mental transformations. Moreover, as Wall [279] observed, raised line drawing has some advantages: they are cheap, have no moving parts, and can easily and cheaply explored. Among the disadvantages, they have limited size, gets worn out and easily worn out. Furthermore, the cost of production of a raised line drawing is fairly low - around US\$ 1.00 per page according to the Virginia Tech Department of Assistive Technologies.

\subsection{First devices built}

We developed and tested devices that fall into both haptic "sub-areas": Reverse joysticks (kinesthetic) and gloves with vibrating motors (tactile). We, however, posit that to successfully navigate to where the teacher is pointing from her original hand position, and understand her, the SBVI must use information gathered from both tactile and kinesthetic senses. For this action to succeed she must be fully aware of where her hand is, and where the device is telling to move her hand. Furthermore, once the navigation phase is over, she will need read the information on the document also using her haptic sense.

Before we move forward and describe how the two haptic devices work, we must introduce three acronyms that will be used extensively throughout the rest of this document. 
1. PIF - Point of Instructional Focus is the location of the teacher's "pointing hand".

2. TPA - Tactile Point of Access is the location of the student's "reading hand" over a raised line drawing.

3. DV - We call the information to be conveyed to the SBVI through our haptic devices, the Disparity Vector - DV. DV has two components: Direction and Distance. With the information contained on the DV, the student should be able to move her hand from the current PTA to the PIF. Once her reading hand is on the PIF, she should understand the teacher.

\subsubsection{The reverse joysticks}

We called them reverse joysticks because they work in a reverse way when compared to those joysticks used in games. Instead of using them to guide a character on the game, for example, the reverse joystick user is guided by the device. So the user rests her hand on top of it and receives directional cues by its displacement. So for example, when the joystick lever moves forward, the student also needs to move her hand forward. The amplitude of the displacement can be used to convey the distance. Note that the user must use one hand to receive directional cues while the other hand does the actual navigation. The reverse joystick idea finds some ground on the work of Guiard's [105. He reported interesting results on the study of the collaborative work of the two human hands. This work was summarized by Mackenzie [177] and shown on table 2.1. By looking at the table, one can imagine that the student who is blind would lay her non-preferred hand on the joystick lever which would direct her preferred hand over the raised line drawing.

Table 2.1: Guiard's model of bimanual control (from Mackenzie[177], page 41). Hand Preferred Action

\begin{aligned} \hline Nonpreferred & - leads the preferred hand. \\ & - sets the spatial frame of reference for the preferred hand. \\ & - performs coarse movements. \\ Preferred $\quad- &$ follows the nonpreferred hand. \\ - & works within the established frame of reference set by the non- \\ & preferred hand. \\ - & performs fine movements. \end{aligned}

Two models of reverse joysticks were tested (Fig. 2.1). There are no important operational differences among them. Basically, Joystick \#1 is bigger, has a longer lever and its motors 
are stronger and one can feel a stronger push when her hand is resting on its lever. Both devices have external power supplies and are connected to the computer via serial cable.

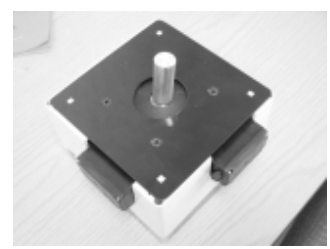

(a) Joystick \#1, larger and stronger than Joystick \#2. It provided a strong push on the users hand.

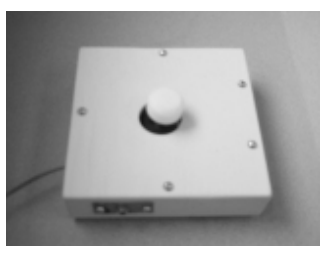

(b) Joystick \#2, smaller and weaker push on the user's hand. No significant difference in usage among both models

Figure 2.1: The reverse joysticks.

Several volunteers tried the reverse joysticks. However, the SBVI need both hands to read tactile information. As Guiard predicted, one for setting the the spatial frame, while the actual fingertip reading is performed by the other. This is harder to do with a joystick than it is with a glove. We, therefore, decided to abandon the use of reverse joysticks.

\subsubsection{The choice for the hands for conveying tactile stimuli}

Several body parts were investigated for receiving tactile stimuli: buttocks [170, 185], head [94], torso [118, 145, 171, feet [95, 133, 165], and hands [35, 237, 275]. Hands have a better "discriminative power" than the rest of the body surface and are represented by a larger portion in the somatosensory cortex than the other body parts involved in touch [202]. Furthermore, hands, along with face, are the skin regions with lowest frequency detection of the human body ([282] pp 195-222), [274]. This is important because it gives the opportunity to convey information (eg. distance) by varying frequency.

Hale and Stanney [109] wrote a survey on haptic literature to identify the "conditions under which haptic displays could enhance human perception and performance". They report that globrous, or hairless skin (eg. palm of the hand) has receptor of four types: Pacinian Corpuscles, Ruffini Endings, Meissner corpuscles and Merkel Disks. These receptors are sensitive to physical parameters like vibration, acceleration, roughness, skin stretch, lateral force, motion direction, static force, velocity, flutter, slip, grip control, skin curvature, pressure, form, texture and edges. The authors point the "glabrous skin, particularly the hands", as the most effective area for "detailed tactile information". Furthermore, they suggest the use of the palm of the hand for "texture and $2 \mathrm{~d}$ form perception". Tactile perception, according to 
the authors, results from a combination of inputs of all receptors of a given skin area, since they are all simultaneously stimulated.

The evidence above presented is probably strong enough to justify the choice of the hand as the body part to receive the tactile stimuli. The question now where on the hands? On the fingers? The ventral or the dorsal part of the hand? How far apart from should the actuators be from each other? When spatial acuity as low as $4 \mathrm{~cm}$ is acceptable, any locus will suffice [282, 143]. Given the rage of sizes of the human hand, an actuator spacing closer to 4 $\mathrm{cm}$ is needed. The two point discrimination threshold (TPDT) is the oldest and simplest measure of tactile spatial acuity. It is usually defined as the minimal distance at which two simultaneous stimuli are distinguishable from a single stimulus [148], ([44] pp 384-385). To obtain the values, the experimenter touches two points of the subject's skin and asks whether she perceives one touch or two. Table 2.2 has a compilation of two point thresholds of different regions of the body. The picture on the same table depicts Sensory homunculus for touch [198 - the body parts are proportional to the area in the brain concerned with their sensory perception.

Table 2.2: Two-point Tactile Thresholds, from Christman's work[44], page 386

\begin{tabular}{ccc}
\hline \hline Sensory homunculus for touch & Region of skin & Threshold, mm \\
\hline & Middle finger & 2.5 \\
& Index finger & 3.0 \\
& Thumb & 3.5 \\
& Upper lip & 5.5 \\
& Nose & 8.0 \\
& Palm & 11.5 \\
& Forehead & 15.5 \\
& Sole of foot & 22.5 \\
& Forearm & 38.5 \\
& Back & 44.0
\end{tabular}

According to the TPDT criterion, the hand also seems to be a good candidate and the palm is, our opinion, the best. Low TPDT is important because glove directional signals should be clearly perceived even by children wearing small gloves. In the following section, we will present some haptic gloves built by other researchers. Some of them chose other parts of the hand to place the actuators. Once we have introduced them, we will make the case for the palm. 


\subsubsection{Other Haptic Gloves}

In this section, we will address how other haptic gloves were built and for which purposes. There are many complex, sophisticated haptic gloves out there. DiPietro and colleagues published a comprehensive survey on glove based systems [67]. In this work, the authors discuss a plethora of applications. Fields as diverse as design, manufacturing and sign language understanding. Several of them are commercially available, like CyberGlove [197], Human Glove [122] and 5DT data glove [65]. All these gloves have sensors to measure flexions and extension of the fingers. Most of them are data acquisition devices and can capture different aspects of hand movement. In our case, we use the glove as a display, to display directional messages. We found only two research projects using haptic gloves for that objective. The first one, by Zelek et al [302, 303] and the second, by Manshad and Manshad [180]

Zelek's 302] glove (Figure 2.2 is part of a portable navigation system. Similar to ours, Zelek's system receives input from a camera. Once the obstacles or other objects of interest are identified, their positions and distances are signaled to the user through vibrations transmitted by the glove. Zelek placed the glove's actuators on the dorsal part of the fingers. Acknowledging that the dorsal part is less sensitive than the ventral, he argues that the fingertips need to be free to read braille. Vibration on the pinky means presence of obstacle to the left; on the index finger, obstacle to the front; on the thumb, obstacle to the right. The stronger the vibration, the closer the obstacle is. Zelek, however, did not reveal whether the users benefited from this last cue.

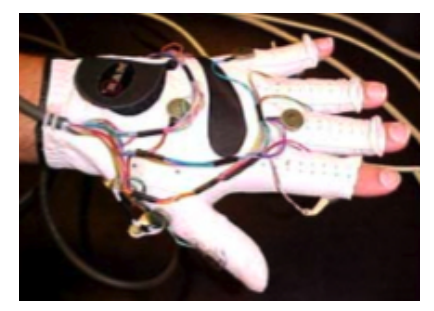

Figure 2.2: Zelek's haptic glove

Manshads' glove (Figure 2.3a) is part of system designed to help the blind in multimodal graph exploration. As Zelek's, Manshads' glove has its vibrating motors placed in on the dorsal part of the fingers. The signalling patterns were also simple and similar to Zelek's scheme, with the difference that there was no change in vibration to convey how close the target is.

Both gloves were successful in conveying direction signals to their users while performing the experimental tasks. This is encouraging but no assurance that we will be equally successful. 


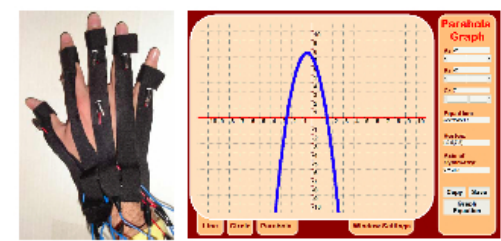

(a) Manshad's Vision Glove and graphing software

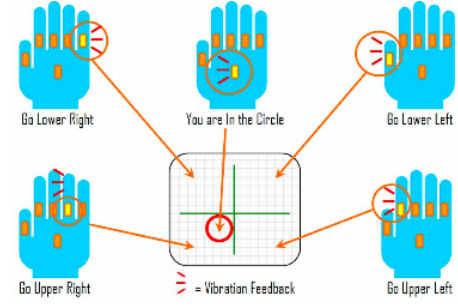

(b) Signaling scheme from Manshad's [180]

Figure 2.3: Multimodal graphic exploration proposed by Manshad and Manshad [180]

The tasks are different. As we pointed out earlier, one of the main differences of our research is that of human to human interaction. Both in Zelek's and Manshad's experimental tasks, there was no dialog involved. As we will discuss later in this document, when people are conversing, there is a natural pressure to keep the discussion flowing [53]. We will return to this topic later in this document when we devise specific experiments to test how conversants adapt and explore the new interactional possibilities the $H D S$ affords. At this point, we want to express our concern about having the actuators placed on the fingers. A quick look at the both gloves (Figures 2.2 and 2.3a) is enough to raise worries about the probable difficulties users might have bending their fingers. We imagine that the student will rest her wrist on her desk and from that position, touch the raised line document before her. This is a more comfortable position and more suitable to withstanding longer periods (eg class time). Should a student adopt such position, she will have to do some of bending. Other important question not answered by the researcher is the existence of any decrease in fingertip sensibility due to the proximity of the actuators. If such problem really exists, asking a blind person to wear these devices would be the same as asking a sighted person to use a blindfold - it would make those projects unfeasible.

Moreover, both Zelek and the Manshads failed to explain why their gloves have that particular actuator arrangement and how they arrived at that specific signaling pattern. Issues like comfort, hand mobility, response time, task analysis, among others, were left untouched. There is a rich body of work that has much to inform us on this matter. We will return for a deeper discussion on these topics after we describe our haptic gloves.

\section{Building haptic gloves - A discussion on core technologies}

A good way of starting this discussion is laying out the core materials and technologies available today for the construction of haptic displays. Note that we do not have the pretension of listing all the core technologies ever used to convey useful information through the haptic sense. The ones shown in table 2.3 should be seen as "potential candidates". The table also 
shows the advantages, disadvantages and publications on each core technology considered.

Cell phone vibro-motors are present on the gloves developed by: Zelek [302] and the Manshads [180]. Regenbrecht 222] sees them as "complicated to control". Since they are motors, there is always the time for them to accelerate to maximum speed and after that, to stop. Van Erp 267] found that the subjects could not distinguish the changes in vibration speeds (used to signal distance to target/obstacle). Other potential problem with the motors is the "unwanted acoustic" [265] - specially if more than one is put to vibrate simultaneously. Van Erp [265] suggests that they should be housed to minimize this problem. This "housing" alternative on the other hand, must be provided in a way to avoid loss of sensibility and distinguishability of the signals emanating from the device. This unwanted acoustic is reason enough to rule out the electromagnetic alarm buzzers and the loudspeakers, despite the fact that they are cheap and easy to control. The problem with thermal displays is time (to heat up and cool down) [196] and the difficulty to convey useful information through temperature differences [144]. Time seems to be an issue for shape memory alloys as well.

Piezo bend bars appear to be an interesting alternative. The bars are very small and bend when submitted to electric current. Without the current, they return to their original form. They do not have the inertia problem of the vibro-motors. This could be an interesting solution. However, building a device with this technology to convey both distance and direction would not be simple. A possible solution for this would be the installation of multiple actuators to convey the same direction; their position with respect to each other would signal the distance. The result could be a device full of actuators packed too close to each other- dangerously close to the minimum distance necessary for two signals to be perceived as two - TPDT.

There are two important issues associated with Raised Pin Displays. First is the fact that they are expensive and their assembly is very laborious [278, 277]. Second is the choice of which pins to raise to convey the $D V$. A student with small hands, for example, would probably have to explore the display to discover which ones were raised. It is important to keep in mind that the student is already required to do tactile exploration - the exploration of the raised line graph. A situation where the student is required to perform two simultaneous tactile explorations is not a desirable.

After this quick review on the available technologies, we are in the position to make the case for the vibrating motors on our haptic display. First, because the now long history of these motors on our daily lives since they are embedded in our cell phones. Furthermore, these motors are easy to find, cheap and reliable. Because of that, the final cost of such gloves is low. Manshad and Manshad reported the cost of their glove was around US\$ 6.00. This is much cheaper than a Phantom, which costs around US\$ 4,000.00 [186, 228] and Logitech's Wingman force feedback mouse US\$60.00 [225, 268]. This low cost gives us the opportunity of testing different glove models with different motor arrangements. Another advantage is that we can build gloves in different sizes to fit different hand sizes. Obviously, if these 
Table 2.3: Core devices used to build haptic displays

\begin{tabular}{|c|c|c|c|c|}
\hline Technology & Figure & Advantages & Disadvantages & Publications \\
\hline $\begin{array}{l}\text { Cell phone } \\
\text { vibro-motor }\end{array}$ & & $\begin{array}{l}\text { Easy to use, } \\
\text { cheap [222] }\end{array}$ & $\begin{array}{l}\text { "complicated to } \\
\text { control in terms of } \\
\text { the amplitude and } \\
\text { frequency of the } \\
\text { vibration", Regen- } \\
\text { brecht }[222 \text { - it takes } \\
\text { time to start/stop }\end{array}$ & $\begin{array}{l}\text { Zelek et al [302], } \\
\text { Manshad and Man- } \\
\text { shad [180] and Yuan } \\
\text { and Folmer [301] }\end{array}$ \\
\hline $\begin{array}{l}\text { Electromagnetic } \\
\text { alarm buzzer }\end{array}$ & & $\begin{array}{l}\text { cheap and easy } \\
\text { to control }\end{array}$ & $\begin{array}{l}\text { limited contact area } \\
\text { in relation to the size } \\
\text { of the device }\end{array}$ & 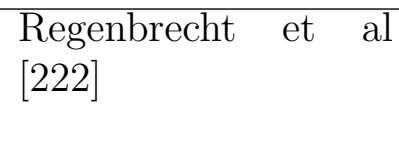 \\
\hline $\begin{array}{l}\text { Piezo bend } \\
\text { bars }\end{array}$ & & $\begin{array}{l}\text { Simple mainte- } \\
\text { nance, small and } \\
\text { flat, very fast } \\
\text { response time } \\
{[222]}\end{array}$ & $\begin{array}{l}\text { dynamics: small dis- } \\
\text { placements require } \\
\text { accurate amplifica- } \\
\text { tion, it needs high } \\
\text { voltage }\end{array}$ & $\begin{array}{l}\text { Regenbrecht et al } \\
\text { [222], and Cholewiak } \\
\text { and Craig [42] }\end{array}$ \\
\hline $\begin{array}{l}\text { Thermal } \\
\text { display }\end{array}$ & & $\begin{array}{l}\text { Used in virtual } \\
\text { reality to en- } \\
\text { hance realism } \\
{[66}\end{array}$ & $\begin{array}{l}\text { Too slow [196], } \\
\text { hard to convey } \\
\text { useful information } \\
\text { through temperature } \\
\text { differences [144] }\end{array}$ & $\begin{array}{l}\text { Jones and Berris } \\
{[144], \text { Monkman et }} \\
\text { al [196] }\end{array}$ \\
\hline Loudspeaker & & $\begin{array}{l}\text { Availability } \\
\text { of more pres- } \\
\text { sure levels and } \\
\text { frequencies } 24]\end{array}$ & $\begin{array}{l}\text { To produce a distin- } \\
\text { guishable vibration, } \\
\text { more power is neces- } \\
\text { sary, which demands } \\
\text { larger devices that in } \\
\text { turn, generate more } \\
\text { noise } 222\end{array}$ & $\begin{array}{l}\text { Bongers and Van Der } \\
\text { Veer [25] and Aka- } \\
\text { matsu and MacKen- } \\
\text { sie [2] }\end{array}$ \\
\hline $\begin{array}{ll}\text { Raised } & \text { Pin } \\
\text { Displays } & \end{array}$ & & $\begin{array}{l}\text { The fact that } \\
\text { is refreshable } \\
\text { makes it an } \\
\text { interesting al- } \\
\text { ternative for } \\
\text { classroom use }\end{array}$ & $\begin{array}{l}\text { Difficult to build, } \\
\text { and higher cost }\end{array}$ & $\begin{array}{l}\text { Wagner and Lede- } \\
\text { man [278, 277] }\end{array}$ \\
\hline $\begin{array}{l}\text { Shape- } \\
\text { memory } \\
\text { alloy }\end{array}$ & & $\begin{array}{lr}\text { It } & \text { provides } \\
\text { larger } & \text { dis- } \\
\text { placements } & \text { in } \\
\text { comparison } & \text { to } \\
\text { piezo bars } & 104] \\
\end{array}$ & $\begin{array}{l}\text { Slow because it takes } \\
\text { time for the wire to } \\
\text { cool down [104] }\end{array}$ & $\begin{array}{l}\text { Grant and Hayward } \\
{[104}\end{array}$ \\
\hline
\end{tabular}


gloves are ever to be mass produced, this low cost would also play a very important role. This low cost fits well with our strategy of iterative development. As we will see in the following sections, our usability studies showed both weaknesses and strengths of our initial glove models. And we responded to those developing new glove. We shall now present our first glove models.

\subsubsection{Initial models of our haptic gloves}

Figures 2.4a, 2.4b and 2.4c show our first generation of haptic gloves. Each one contains a set of vibrating motors ${ }^{1}$ arranged in a specific configuration. Configuration $A$ has a rectangular shape (Figure 2.4a) with its twenty actuators arranged in a $5 \times 4$ grid; Configuration $B$ has its actuators assembled as in a square (Figure 2.4b), in a 4x4 grid. Finally, Configuration $C$ has 12 motors set up as the hours of an analog clock (Figure 2.4c).

A controller box with a PIC18F452-I/P and a set of driving transistors control the firing patterns of these motors (Figure 2.4d). The gloves are connected to the controller box via serial cable. The controller box is, in turn, connected to a computer also via serial cable. The program in the microcontroller controls the timing and intensity of the vibration of each individual motor on the gloves. Each motor or actuator has a unique address, and its vibration intensity has sixteen different levels. The PIC18F452-I/P has 4 independent 8-pin output control registers for simultaneous control of up to 32 devices. We employ the pulse width control mechanism of the microcontroller to produce a sense of varying intensity. The microcontroller can set the motor to vibrate at its highest intensity simply by providing the motor current long enough so that it reaches full spinning speed. Setting vibration intensity to zero brings a motor to a halt.

During pilot tests, we found that the wires within the gloves are too thin and could easily break. They could break with normal manipulation - people trying to put the glove on or using it. At that point we figured out that we needed our gloves to be robust. In response to that, we decided to house each motor into small and individual pockets. This would at least increase its maintainability as motors that stopped working could be easily changed without the need to redo all the wiring. This second generation glove (shown in Fig. 2.4d) also had straps coming out of its sides so that the user could adjust it to a better fit to the palm.

Now that we have introduced our glove, we shall discuss relevant aspects our device with respect to signal conveyance.

\footnotetext{
${ }^{1}$ See appendix A.2 on page 181 for the vibrating motor specification
} 


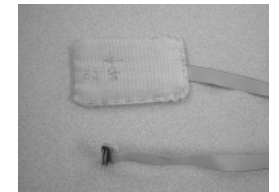

(a) The original "square" model. The actuators were arranged on a $4 \times 4$ grid

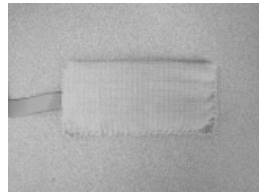

(b) The original "rectangular" model. The actuators were arranged on a $5 \times 4$ grid.

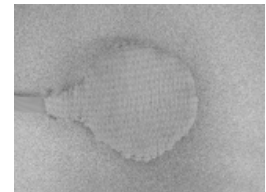

(c) The original "round" model.

The actuators were arranged as the hours on an analog clock.

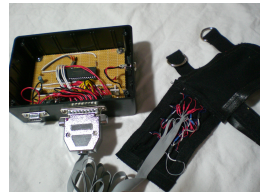

(d) Second generation glove and the controller box

Figure 2.4: First and second generations of haptic gloves.

\subsubsection{Tactile perception threshold}

Since we have chosen vibro-tactile motors, it is given that its vibration intensity is higher the human threshold for tactile stimuli. According to Bolanowski and colleagues [22], the detection threshold for vibrotactile stimulation is roughly $28 \mathrm{~dB}$ (re 1 micron) below $30 \mathrm{~Hz}$ and decreases at a rate of roughly $-12 \mathrm{~dB} /$ oct from 30 to $300 \mathrm{~Hz}$. In our glove, these motors are put into copper tubes, which are in turn, put into pockets. The glove assembling process decrease the motors' vibration because both the copper tubes and the pockets reduce the room for them to move.

We measure vibration in terms of acceleration, which in turn is measured in $\mathrm{m} / \mathrm{s}^{2}([232]$ p 591). Accelerometers are normally used to measure vibration. However, we cannot put accelerometers on the gloves once they are assembled. Griffin ([232] p 591) offers an alternative: If displacement $\mathrm{d}$, and frequency $\mathrm{f}$, are known, acceleration, a, can be calculated as follows (according to Griffin ([232] p 591))

$$
a=(2 \cdot \pi \cdot f)^{2} \cdot d
$$

Once acceleration is known, it can be converted to Decibels. A simple conversion formula according to Griffin ([232] p 591) is :

$$
a_{d b}=20 \cdot \log \frac{a_{m / s^{2}}}{a_{0}}
$$

Where $a_{0}$ has a constant value of $10^{-6} \mathrm{~m} / \mathrm{s}^{2}$, which is the International Standard 1683 (ISO, 1983).

We still have problems in trying to measure the nominal acceleration of our device. Frequency is given by the motor specification sheet equals 11000 RPM or $183.33 \mathrm{~Hz}$. However, the motor displacement within the glove is also very hard to measure. 
Even if we could obtain these data, we would still have to face issues like how tight the glove is strapped to the user's hand. This "tightness" clearly plays a role on the motor acceleration and displacement within the glove. How tight the user fastens her glove is a tradeoff between comfort and perceptual salience [148]. Because of all the issues discussed above, we stopped trying to find the nominal vibration of our device and later compare it to the available literature. We simply went straight to the usability tests described later in this document.

\subsubsection{How to convey the Displacement Vector $-D V$}

Now that we have the device, we need to devise a way of using it to send our Disparity Vector - Direction and distance. Again, we investigate previous works to learn what worked and what did not.

Tan et al 259] designed a 3-by-3 vibrotactile array that was sewn between two supporting layers of fabric so they can be draped over the back of an office chair. With this configuration, eight directions could be sent: East, West, Southeast, Southwest, Northeast and Northwest. In their experiments, participants were able distinguish the directional signals without been briefed on them. Mapping from the sensory experience to the direction itself can be easily learned [148. Tan and colleagues' device design influenced us. It was simple (only eight vibrating motors), seemed effective and produced easily perceivable signals. Van erp [267] also employed the "eight directions strategy" to his waist vest and equally reports good results. We decided to follow the same strategy.

The strategies discussed above seem simple and are proven to work. However, we need to be cautious. First, because it is a different device, to be worn in a different body part. Second, because it will be used in a different task - attending mathematics lectures. Again, we emphasize that the haptic aided navigation must not compete for attentional resources with attending the lecture. For that, we need maximum perceptual salience and that is worth a deeper investigation.

In his Guidelines for the use of vibro-tactile displays in human computer interaction, Van erp [265] suggests the examination of four parameters related to vibro-tactile perception: magnitude, frequency, timing, and location. We shall discuss each parameter under the light of our specific device/task.

Magnitude is related to the intensity level of the vibration. Craig [57] showed that it is possible for the user to perceive different levels of vibration and therefore using such differences to convey different information seems feasible. Van Erp [265], on the other hand, suggests caution in the use of this parameter. He advocates the use of no more than four different levels of vibration. A potential use for the parameter is to signal the target distance. Van Erp used it in his waist vest 267] but his participants could not benefit from it. Tan and colleagues did not use different vibration intensities. During our pilot testing, we could distinguish 
several levels of vibration intensity. However, we decided to followed Van Erp's advice and be very economical on this matter: Only one level was used - the highest. The argument is simple. The different levels were useless when it came to signaling distance, according to Van Erp's experiment. We need the maximum signal salience to facilitate discrimination and boost task performance. As Van Erp posited, direction is more important than distance.

It is hard to distinguish frequency from magnitude in vibro-tactile stimulus [96]. It is nearly impossible to make such distinction using the type of motors we use. In our case, it all comes down to the time electric current is sent to the motor. If it is long enough, the motor will spin at its full speed. That means that at top speed, the magnitude will be at its peak and so will the frequency. In all cases, both parameters go hand in hand. We therefore refrain to make any distinction between them. From this point on, we refer to both parameters as intensity level meaning how the human user perceives the level of magnitude/frequency.

The temporal sensitivity of the skin is higher than that of vision [251. Humans can perceive two vibro-tactile stimuli as two, as long as they are separated by an interval as short as $10 \mathrm{~ms}$ [90, 213. Here, our choice for cell phone vibrating motors takes its toll. In our preliminary studies, these motors have to receive current for at least $30 \mathrm{~ms}$ to achieve its maximum speed. What is the impact of such delay in the user's response time is one of the questions we will try to answer in throughout this document. For now, we must acknowledge our concern on this matter due to the finding of Jay and colleagues [136]: feedback delays as short as 50ms are sufficient to impact task performance. We posit, however, that the greater risk of task performance problems is related to how soon the user can distinguish the signal and not how fast the motor can reach full spinning speed. Besides, since we are dealing with human to human communication, it is known that humans have a remarkable adaptation capability when it comes to novel ways of interaction [164]. It is early to tell whether this delay will play any significant role in the overall communication performance.

Temporal enhancement occurs when a stimulus on a different part of the body replaces the current one. When such replacement happens, the second stimulus is perceived with a greater magnitude than the "old" one [273]. Such phenomenon can benefit the student during actual interaction with the teacher. During navigation, the second signal will always indicate the new direction to move the hand. The sooner the student is aware of that the better.

Still, there is the temporal summation phenomenon. When the vibrotactile stimulus time increases, the threshold drops [272], increasing the possibility of detection. To benefit from this finding, we decided to keep the glove vibrating at all times. However, as we will see later in this document, the user will always be able to stop and resume the vibration whenever she wants.

Another interesting phenomenon in tactile signaling is the sensory saltation - first observed at the Princeton Cutaneous Communication Laboratory. The researchers put three mechanical tactors on the participant's forearm and delivered 3 short pulses to 3 different positions on the arm. The first position was closer to the wrist, and the third position was closer to 
the elbow. The second position is in midway from the first to the third position. "Instead of three taps, participants reported a feeling similar to a little rabbit hopping from the wrist to the elbow", reports Geldard [87] (Figure 2.5).

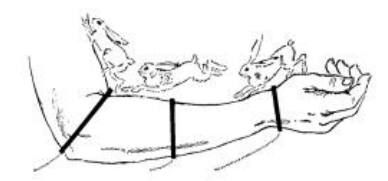

Figure 2.5: A Norwegian newspaper cartoonist's illustration of "sensory saltation" [87].

Kirman [158] also observed apparent movement increases as a power function of increasing in stimulus duration. The "hopping rabbit" is a good way of signaling direction. All the user needs to do is "follow the rabbit" to understand the direction to move his hand. We reproduced the phenomenon during our pilot testing.

Because numerous combinations of values can be assigned to the parameters above discussed, we decided to store them in data files, what we call pattern files. Whenever we need to change the vibration patterns, we edit those plain text files. Each glove model has its own file. In case we need a new glove model, we just create its corresponding pattern file. The pattern is stored as a list of 3-tuples. Each 3-tuple represents an 'activation command' by specifying the actuator ID, the intensity of vibration, and the time delay before performing the next command. A time delay of zero milliseconds represents simultaneous activation. For example "5 $100,71030,380,4810,500,7030,300,400$ " tells a glove to fire up actuators 5 and 7 to intensity 10 simultaneously, wait $30 \mathrm{msec}$ and then fire up actuators 3 and 4 at intensity 8, wait $10 \mathrm{msec}$ and stop actuators 5 and 7, and wait $30 \mathrm{msec}$ then stop actuators 3 and 4.

We tried several different patterns for each direction on each glove to find one that produces a strong, clear, and short signal. We wanted to increase signal salience, and therefore reduce memory load (291] p 41). The lesser memory load the better our chances to enable multimodal interaction. During pilot studies, we first tested the saltatory pattern. In the $5 \times 4$ glove, for example, "North" was conveyed as vibrating actuators 18, 13, 8, and 3 sequentially at intensity 10 for $30 \mathrm{msec}$ each, with a short $5 \mathrm{msec}$ pause in between. This is specified in our data file as "18 10 30, 180 5, 1310 30, 130 5, 810 30, 80 5, 310 30, 30 0" to produce the virtual upward motion shown in Figure 2.6.

The direction was clearly perceived when signaled via the saltatory pattern. However, this approach costs some precious milliseconds to completely send a directional signal. The startstop interval between the vibrations has to be long enough to allow the sequencing to be perceived. This pattern, although very easily perceived, was abandoned because of the long duration of the signal. To keep the signal short, we decided to pick the smallest number 


\begin{tabular}{|c|c|c|c|c|}
\hline 1 & 2 & 3 & 4 & 5 \\
\hline 6 & 7 & 8 & 9 & 10 \\
\hline 11 & 12 & 13 & 14 & 15 \\
\hline 16 & 17 & 18 & 19 & 20 \\
\hline
\end{tabular}

Figure 2.6: A possible way to convey North using $5 \times 4$ glove

of actuators necessary to convey a direction and vibrate them all together at the highest intensity possible. For this, we chose the actuator or actuators with most significance for a given direction. The most significant actuator for the North direction would be the one with address three in figure 1. South would be actuator 18. For Northwest, we used actuator 1. For East and West directions, we chose to vibrate actuators 6, 11, and 10, 15, respectively. For the vibration duration, it was empirically set to 30 milliseconds. Less than that, the motors will not spin at full speed, making the vibration less perceivable. Once we had the signaling pattern decided, we move to the experimental phase of our glove, looking for the answers we couldn't find in the literature.

\subsection{The Experiments}

The literature can only help us that much. Much of the data we used as reference were obtained in laboratory in settings close to ideal. When we actually build a new device it is important to design our own battery of tests. In our case, we approach the issue with a series of experiments that will increasingly resemble the scenario of a blind student attending class. With this in mind, we enumerated a list of questions we think are relevant to this stage of our research.

1. To what extent can the gloves convey the sense of direction?

(a) What vibration patterns are best suited for conveying direction?

(b) How to cope with the inertia?

(c) How to cope with the unwanted acoustic?

(d) Is there any region, in the palm, where the sensibility is greater?

(e) Due to the proximity of the motors (in the palm), will the participants be able to distinguish the signals coming from the different motors? 
(f) How fast will the users respond to the stimulus onset? Will this response be suited to human to human interaction (if the response is too slow, the conversants might think that the device is "getting in the way" of their conversation).

2. Can the user read braille while the motors are vibrating?

(a) Will the glove pose any extra difficulty in reading?

3. Can the user navigate while listening to a story?

4. Does hand dominance play any role in perceiving the tactile stimuli our devices convey?

5. Will any improvement be observed as the participants are exposed to the haptic experience?

6. Will fatigue be observed?

Some of the questions were be answered via experiment design. In other words, the experiment is designed to answer that specific question. This is the case for the ones we consider the most important. We tried to answer the reminder through the analysis of the data collected. All trials were videotaped. Data were also automatically collected by the programs we built specifically for the experiments. Participants were also required to answer a questionnaire before the experiments, and other after.

Twenty-five members of the Virginia Tech community participated (19 males and 6 females) helped us throughout three experiments performed in a single hour-long session. Their age ranged from 21 to 52 years old and averaged 29.35. One participant could not complete experiments two and three, but we did collect data from her experiment one trial. We tested the three glove models on each hand (dominant and non-dominant) four times. We wanted each participant to perform all three experiments so that we could track progress. It took one hour for one condition (one hand, and one glove configuration) alone, making it impractical to test our subjects on all gloves and both hands. Hence, the participants wore the same glove model on the same hand throughout all three experiments. The trials were counterbalanced.

It is important to notice that none of the participants were blind. It is believed that blindness is associated with superior non visual perception. It is consensus that any advantage of the blind is due not to heightened sensitivity, but rather to the development, and refining of perceptual skills with practice [234]. The basis for such practice-related perceptual improvement is the remarkable plasticity of the Central Nervous System [234]. Pascual-Leone and Torres [211] used somatosensory evoked potentials and transcranial magnetic stimulation (TMS) to demonstrate that the Braille-reading finger has an expanded representation in sensorimotor cortex of blind Braille readers. Van Boven [264] et al report that grating resolution at the fingertip is nearly $30 \%$ percent better in blind Braille readers than controls with normal vision. That study used gratings consisting of alternating ridges and grooves 


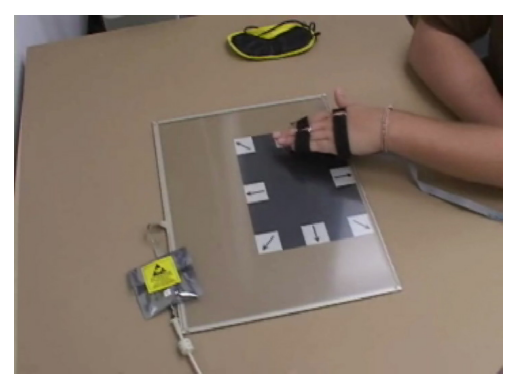

\section{Figure 2.7: Experiment I - A participant choosing the direction he perceived}

that were impressed into the fingerpad oriented either along or across the long axis of the finger.

Blind Braille readers can identify Braille-like dot-patterns almost 5\% more accurately than sighted subjects [79]. Steven et al [255] show that the blind can detect gaps that are over $15 \%$ narrower, distinguish the orientation of lines that are nearly $40 \%$ shorter, and discriminate dot-patterns bearing minute spatial offsets in the hyperacuity range (i.e., below the limits of spatial resolution) with about $50 \%$ lower thresholds. With practice, however, sighted subjects can match the tactile performance of the blind [103].

We decided to use normals for this phase of our research and not overuse our limited number of SBVI. This permits us first to establish a baseline of the efficacy of the devices over a larger population. We expect that the blind subjects would perform better in the tasks. We also understand that the findings described on this chapter can be applied to the blind.

Participants had a chance to practice before each experiment.

\subsubsection{Experiment I}

This experiment was designed to answer the question: To what extent can the gloves convey directional information? Eight direction signals were randomly sent thorugh the glove. Before each trial, the experimenter asked the participant whether she was ready to receive the signal. Upon an affirmative answer, the experimenter clicked a button to start sending the signals. The participant was asked to quickly and accurately tap on the arrow corresponding to the perceived direction on a touch-sensitive pad (Figure 2.7). This avoids the problem of lexical access where the participant has to think of the "name of the direction". The graphical direction presentation on the horizontal tabletop also reduces the problem of coordinate transform for the subject and simulates the orientation of reading a raised-line graphic.

To increase redundancy and reduce confusion, we arranged the display so that the arrows' locations correspond to the directions on a map ([291] p 47), [46]. We recorded the direction sent, the direction perceived, and the participant's response time. 
Table 2.4: Response time in seconds per glove model

\begin{tabular}{|c|c|c|c|c|c|}
\hline $\begin{array}{c}\text { Glove } \\
\text { Model }\end{array}$ & Trials & $\begin{array}{c}\text { Mean } \\
\text { in Sec }\end{array}$ & $\begin{array}{c}\text { Std } \\
\text { Err }\end{array}$ & $\begin{array}{c}\text { Lower } \\
\mathbf{9 5 \%} \\
\text { in Sec }\end{array}$ & $\begin{array}{c}\text { Upper } \\
\mathbf{9 5 \%} \\
\text { in Sec }\end{array}$ \\
\hline \hline $4 \times 4$ & 55 & 0.592 & 0.047 & 0.499 & 0.686 \\
\hline $5 \times 4$ & 48 & 0.553 & 0.050 & 0.453 & 0.653 \\
\hline Round & 40 & 0.544 & 0.055 & 0.434 & 0.653 \\
\hline
\end{tabular}

Table 2.5: Confidence Interval for hit percentages

\begin{tabular}{|c|c|c|c|c|c|}
\hline $\begin{array}{c}\text { Glove } \\
\text { Model }\end{array}$ & $\boldsymbol{n}$ & $\begin{array}{c}\text { Mean } \\
\text { Error }\end{array}$ & $\begin{array}{c}\text { Std } \\
\text { dv }\end{array}$ & $\begin{array}{c}\text { Lower } \\
\mathbf{9 5 \%}\end{array}$ & $\begin{array}{c}\text { Upper } \\
\mathbf{9 5 \%}\end{array}$ \\
\hline \hline $4 \times 4$ & 9 & $79.16 \%$ & 5.50 & 67.75 & 90.57 \\
\hline $5 \times 4$ & 8 & $78.12 \%$ & 5.83 & 66.02 & 90.23 \\
\hline Round & 8 & $39.06 \%$ & 5.83 & $\mathbf{2 6 . 9 5}$ & 51.16 \\
\hline
\end{tabular}

\section{Response time results}

All studies reported on this chapter were performed in between subjects. We ran one-way Anova at a $95 \%$ confidence interval on the data collected. We also use the same confidence interval for the analysis of experiments II and III reported in the following sections. We can see in table 2.4 that none of the models yielded shorter response times. The results are quite similar. We understand that, with practice, these numbers would decrease.

The participants filled out a questionnaire after they finished the experiment. In one of the questions, we asked them to grade how well they distinguished the directions. We call this number level of reported perception - LRP. Possible LRP values ranged from 1 through 5, 1 being the lowest. The mean response time among those who reported higher LRP (5) was 0.3312 seconds and significantly faster than those who reported lower LRPs $(\mathrm{df}=22$, $\mathrm{f}=3.1868, \mathrm{p}<0.0258$ ). We could not find any significant difference between wearing the glove on the dominant hand and the non-dominant hand.

\section{Perception results}

Figure 2.8a shows the results for the gloves in terms of correctly perceived directions per glove model (hits). The $\mathrm{x}$-axis shows the glove models. The $\mathrm{y}$-axis shows hit percentages. If the participant could not feel the direction, she would be guessing and right on $1 / 8$ th or $12.5 \%$ of the trials. The value on bold on table 2.5 (Confidence interval for hit percentages) is the worst case: The lower end of the $95 \%$ confidence interval is $26.95 \%$. From this we can 


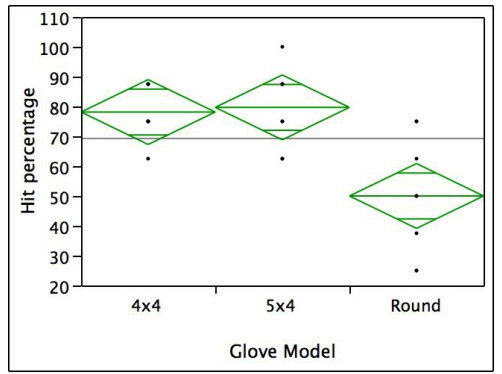

(a) Hit percentages per glove model

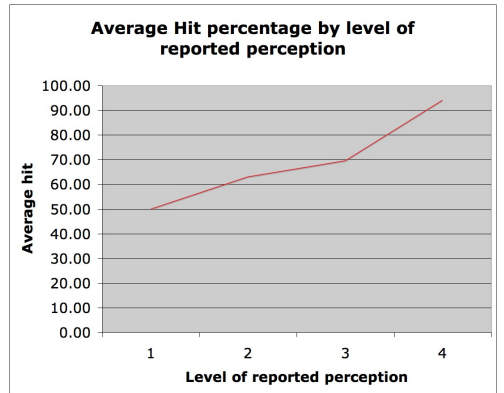

(b) Average hit percentage by level of reported perception

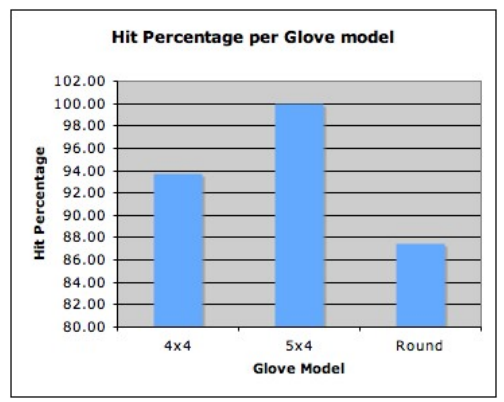

(d) Hit percentage per glove model

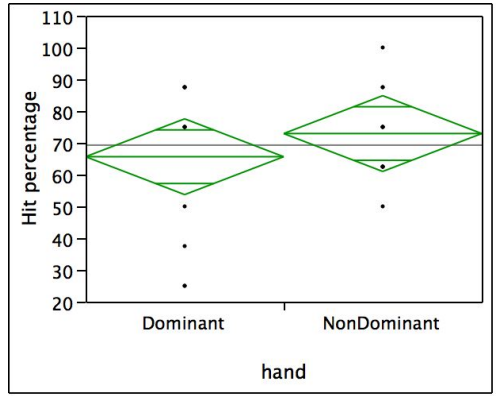

(c) Hits and misses by hand dominance

Figure 2.8: Charts from Experiments I and II

infer that the gloves, at different rates, do deliver the sense of direction.

We cannot conclude that glove $4 \times 4$ performed better than the $5 \times 4$ model. However, the round glove yielded significantly worse results.

The level of reported perception also appears to play an important role on the number of hits. Figure 2.8b shows that participants who had higher levels of reported perception performed better with on this task.

It seems that it does not matter if the glove is worn on either hand (Figure 2.8c). A closer look tells us that the glove worn on the dominant hand performed slightly worse than on the non-dominant hand. However, this difference is not statistically significant.

\subsubsection{Tracking}

The next two experiments required a little more complex computer program. One capable of tracking the users hand and signaling, through the glove, the $D V$. On top of that, the glove 


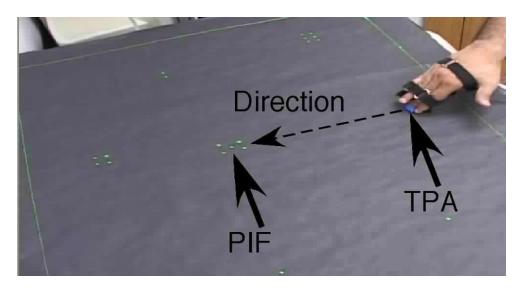

Figure 2.9: Focal Disparity (direction) computed from TPA and PIF

also needs to signal the user when she reaches the PIF. In this section, we describe how this program works.

A downward looking camera tracks the participant's TPA. The camera feeds the system at thirty frames per second. For the experiments described here, we had two fixed targets or Points of Instruction Focus - PIFs [218]. The PIFs had fixed coordinates. For each frame received, the system calculates the direction from the current TPA to the next PIF. Once the direction is found, we convey it through the glove. To keep tracking and signaling as independent as possible from each other, they were implemented as two different computational threads. It might occur that the TPA changes its position while a signal is still being sent. This could occur due to quick hand movement or if the signal is too lengthy. If this happens, the system aborts that signaling and starts sending the new direction. This dynamic tracking approach avoids the "piling up" of old directions that make no sense.

For the experiments, we used a $2.33 \mathrm{GHz}$ Intel Core 2 Duo Mac Book Pro with 2 GB RAM to which the firewire camera and the controller box for the gloves were connected.

For the tracking, we employed the Lucas-Kanade tracking algorithm [26, 175] (we used the OpenCV [126] implementation). The frames captured had 640x480 pixels, the camera was set at approximately $1.5 \mathrm{~m}$ above the desk. For efficiency, we used a 6-level processing pyramid. We ensured accurate and timely tracking by asking the subjects to wear a blue tape on top of their finger and the PIFs were painted green on a black sheet of paper (Figure 2.9).

We created an imaginary circle with 10 pixels of radius with origin at the PIF's coordinates. We considered participant to have reached the PIF when she entered this circle.

\subsubsection{Experiment II}

Experiment II was designed to answer the question: Does the vibration interfere with fingertip reading? This interference may come from the vibration of the motors on the palm of the hand and adjacent areas. Figure 2.9 shows a typical board used in this experiment. We assigned two PIFs on the board for each participant. The glove helped the subject's navigation to the PIF. When the TPA entered the PIF area, the experimenter asked the participant what number she was reading. Each board had six numbers, collection of dots 
Table 2.6: Speed $(\mathrm{cm} / \mathrm{sec})$ in different glove models

\begin{tabular}{|c|c|c|c|c|c|}
\hline $\begin{array}{c}\text { Glove } \\
\text { Model }\end{array}$ & $\boldsymbol{n}$ & $\begin{array}{c}\text { Mean } \\
\text { Error }\end{array}$ & $\begin{array}{c}\text { Std } \\
\text { Dv }\end{array}$ & $\begin{array}{c}\text { Lower } \\
\mathbf{9 5 \%}\end{array}$ & $\begin{array}{c}\text { Upper } \\
\mathbf{9 5 \%}\end{array}$ \\
\hline \hline $4 \times 4$ & 13 & 3.557 & 0.591 & 2.354 & 4.7619 \\
\hline $5 \times 4$ & 12 & 3.107 & 0.615 & 1.854 & 4.3608 \\
\hline Round & 12 & 2.541 & 0.643 & 1.232 & 3.8503 \\
\hline
\end{tabular}

forming numbers like those on a dice. We chose this representation because seeing people are not used to Braille, but normally are familiar to dice or dominoes.

Participants were aware that there were six numbers on the board. Therefore, if they guess they would be correct $1 / 6$ th or $16 \%$ of the times. Figure $2.8 \mathrm{~d}$ shows the means at $95 \%$ of confidence interval. Y-axes show the correct answers (hits) - the participants correctly answering the question.

None of the glove had the $16 \%$ value included on their confidence interval. The results do not allow us to conclude that any glove model outperformed other. We also could not find any significant difference on wearing the glove on the dominant or on the non-dominant hand.

The question this experiment tried to answer is very important for future research on the area. If we have found an important decrease on fingertip sensibility due to the use of these gloves, we would probably be forced to abandon this technology.

We also analyzed the times the participants took to reach the PIFs. A good system would help the participant to quickly get to the PIF.

We ran one-way Anova to try to identify if a particular glove model yielded faster speed (table 2.6). The results were inconclusive. Non-significant differences between the use of gloves on either dominant or non-dominant hand were found.

Moreover, we compared speed and distance to PIFs. We wondered if longer distances would mean slower speeds once the participant would proceed slower because the PIF was further away. Would this decrease the participant's confidence on the signal he was receiving? The answer seems to be $N o$. According to the regression performed on the data, differences on distances explain only $15.11 \%$ of the differences on speed.

\subsubsection{Experiment III}

The experiments get more complex as they get more similar to what is expected in a classroom. In that setting, the SBVI will have to navigate with the help of the system while paying attention to the instructor. Thus, the research question at that point was: To what 
Table 2.7: One of the five stories

A friend is thinking of moving out of his $\ll 2 \gg$ bedroom apartment. He
found another apartment for almost the same price but with $\ll 3 \gg$ bed-
rooms. He hasn't come to a decision yet because the current apartment
is in a very beautiful place. He has until the end of the month to make
up his mind, or face a 5 percent increase in the rent and still live in the
same place.

extent can a person fuse the information obtained from both modes?

To answer this question, we told five different one-paragraph-long stories to each participant. The stories refer to numbers, which are displayed on the boards similar to the one shown on Figure 2.9. Table 2.7 has one of the stories. The experimenter did not say the numbers two and three that appear on the text. On the other hand, number five (5\%) was said. He told the story while the participant navigated to the PIFs similar to what she did on experiment two. The experimenter adjusted the story telling speed according to the participant's distance to the PIF. When the participant reached the PIF, glove stopped vibrating, indicating the user that she has reached the PIF. After a 2 sec pause, the glove started vibrating again indicating the direction to the next PIF. By that time, the experimenter resumed the story telling. At the end of each story, four questions were asked. The experimenter read the questions and the alternatives.

To correctly answer the questions, the participant needed information that was not present on speech; she had to acquire it from tactile reading. Again, if the participant were guessing, she would get only $1 / 4$ th of the answers right. To discourage guessing, we also presented a 5th option; "I don't remember", which was computed as wrong.

In this scenario, we have two tasks competing for attentional resources but using different modes. Wickens (291 p 106) found that cross-modal time sharing is better than intramodal. Joeong et al [140] found good recall rates with users working on auditory-haptic displays. To measure the cross-modal time sharing degree, we asked the participants to grade (from 1 through 5 - one being the lowest) how well they could listen to the story while the glove was vibrating. We call this, the cross-modal time sharing degree.

We ran one-way Anova on the data collected. Again, we found no significant difference among the glove models nor between dominant and non-dominant hand. Figure 2.10a shows the average number of correct answers obtained from participants wearing different glove models. Although we cannot say that a particular glove yielded better performance, we can see that the lowest end of the confidence interval is $67.00 \%$ (bold value in Table 2.8) of correct answers. This number is way above the $25 \%$ expected for guessing. 

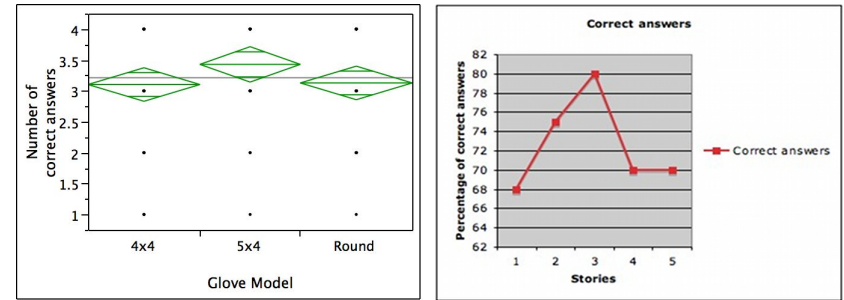

(a) Average of correct an-(b) Percentage of correct swers per glove model answers as stories were told

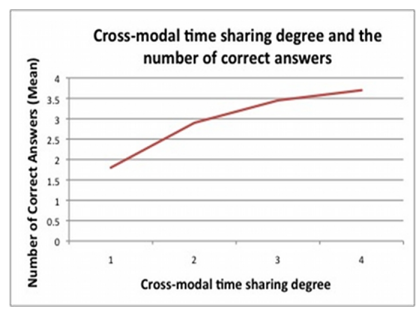

(c) Correct answers per cross-modal time sharing

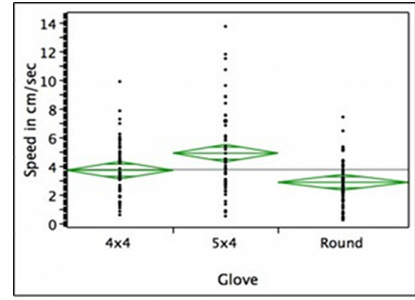

(d) Average of speed per glove model

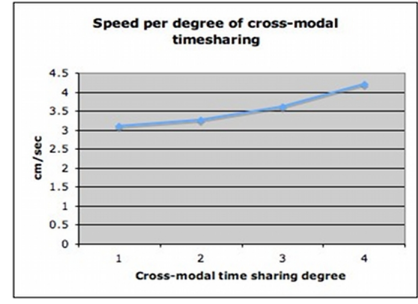

(e) Speed per-cross-modal time sharing

Figure 2.10: Charts from Experiment III

Table 2.8: Averages of correct answers by glove model

\begin{tabular}{|c|c|c|c|c|c|}
\hline $\begin{array}{c}\text { Glove } \\
\text { Model }\end{array}$ & Trials & Mean \% & $\begin{array}{c}\text { Std } \\
\text { Error }\end{array}$ & $\begin{array}{c}\text { Lower } \\
\mathbf{9 5 \%}\end{array}$ & $\begin{array}{c}\text { Upper } \\
\mathbf{9 5 \%}\end{array}$ \\
\hline \hline $4 \times 4$ & 8 & $77.66 \%$ & 0.051 & $\mathbf{6 7 . 0 0 \%}$ & $88.31 \%$ \\
\hline $5 \times 4$ & 8 & $84.79 \%$ & 0.051 & $74.13 \%$ & $95.44 \%$ \\
\hline Round & 8 & $78.59 \%$ & 0.051 & $67.94 \%$ & $89.25 \%$ \\
\hline
\end{tabular}

We also wondered if the participants improved their performance as more stories were told. We plotted the percentage of correct answers per story, consolidating data from all hand/glove combinations (Figure 2.10b). A possible explanation to this graph would be a learning curve followed by fatigue. This was the third experiment, and the participants were performing different tasks for almost one hour. Interestingly, subjects who reported higher degree of cross modal time sharing (5) had a significant higher average of correct answers $(\mathrm{df}=23, \mathrm{f}: 13.9564, \mathrm{p}<0.0001)$ (Figure 2.10c).

We also compared the navigation speeds per glove model (Figure 2.10d). The round glove performed significantly worse than the other models.

We observed a constant and consistent increase of the navigation speed as the participants listened to the stories. It is also interesting to observe how the speed relates to cross-modal time sharing (figure 2.10e - the higher the degree, the higher the speed. However, speed explains only $6.05 \%$ of correct answers, according to the regression performed. 


\section{Post-Questionnaire}

After the all three experiments, the participants answered a questionnaire. We discuss the results in the section. The questionnaire can be found in appendix A.4, on pagepage 185.

Sixty percent said they felt comfortable wearing the gloves, considering all models together. The percentages per glove were: $50.00 \%, 88.88 \%$ and $37.75 \%$ for the $5 \times 4,4 \times 4$, and round gloves respectively.

Fifty five percent affirmed they could perfectly feel the direction. The percentages per gloves were: $62.50 \%, 88.88 \%$ and $12.50 \%$ for the $5 \times 4,4 \times 4$, and round gloves respectively.

Forty eight percent could not tell whether they would perform better if they wore the glove on the dominant hand. The percentages per gloves were: $62.50 \%, 55.56 \%$ and $25 \%$ for the $5 \times 4,4 \times 4$, and round gloves respectively.

Seventy five percent could keep listening to the stories while they navigate. The percentages were: $87.50 \%, 62.50 \%$ and $75 \%$ for the $5 \times 4,4 \times 4$ and round gloves respectively.

\subsection{Discussion}

In summary, the experiments above described show that:

1. The gloves convey sense of direction;

2. The gloves do not interfere with fingertip reading;

3. A person can navigate with the help of this system while listening to a story;

4. It is possible to fuse the information received from both modes. In the next chapter, we detail and justify the actions done in response to these findings.

The results seem encouraging, but could they be better? How can we improve the system? What conclusions can be drawn from the experiments?

We begin this discussion with the glove models. We built only one size per glove model. One participant has such a small hand that it was impossible for her to distinguish any direction. We had to find her a substitute. We certainly had some less extreme fit problems among the other participants. We needed gloves in different sizes.

This "fit" problem must be analyzed more thoroughly. Johansson et al [146] studied the distribution of tactile units in the glabrous skin area of the human hand. We can see in Figure 2.11 that some areas of the hand have more receptive centers than others. We cannot 


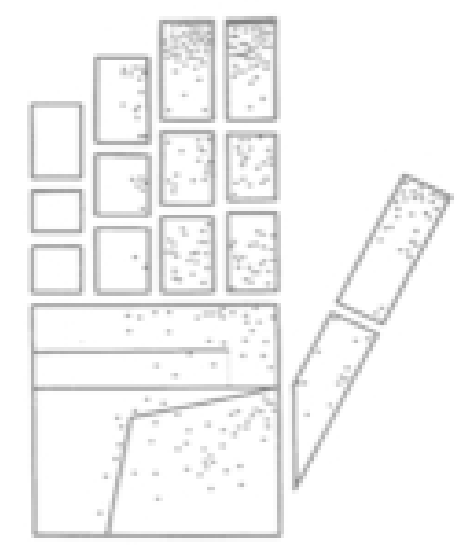

Figure 2.11: Locations of the receptive field centers of 334 globrous skin mechanoreceptive units, from Johansson [146]

place the actuator too close to one another because this would make harder to distinguish among directions (291] pp 87-88). This called to another cycle of research and development.

From the present study, it is not statistically possible to conclude that better results were obtained from the participants who had a better actuator/skin area contact. However, in all tasks, those who reported higher degrees of perception obtained better results. Furthermore, one source of research this study did provide was a confusion matrix (appendix A.1). Analyzing this matrix, we saw that in one fourth of the times North was perceived as Northeast on the $4 \times 4$ glove. Northeast, East and Southwest had a pretty bad hit rate for the round glove. Maybe this is because the actuators responsible for delivering these directions on that model were contacting a poorly in-nerved skin area or not contacting at all.

It is interesting to see that although only $37.75 \%$ of those who tested the round glove felt comfortable wearing it, and only $12.50 \%$ of them could not perfectly feel the directions, seventy five percent of them could listen to the stories while navigating. The data conform to the performance of this group on the third experiment: They correctly answered the questions about the stories over $75 \%$ of the time.

We also could not find any significant performance difference between those who wore the glove on the dominant hand and the ones who wore it on the non-dominant hand.

In the third experiment, the experimenter had to adjust the speed of his speech to participant's navigation speed. This suggests that we need some way to inform the teacher that there is someone in the audience that is not keeping up with the lecture.

We also need to improve the tracking system. The Lucas-Kanade tracking algorithm is based on optical flow which is not stable enough when the object being tracked moves fast or moves out of the scene. We also found an orientation issue. To maintain the consistency between the real world direction and that being sent by the glove, the user must keep his finger in an 
orthogonal position with respect to the desk where the instructional material lays.

The experiments showed promising results but many issues need to be addressed. First, there is the problem of the glove per se. All models were too fragile and several participants had a hard time deciphering the signals they conveyed. We tried to show the correlation between the capability of distinguishing the signals and user performance. Because of this finding, we decided to more deeply investigate alternative glove designs and test them.

\subsection{The need for new glove models}

As a response to the first findings, we have developed and tested several "generations" of haptic gloves. At the end of the studies reported in this chapter, we have arrived at a glove model that:

1. is more robust than its "predecessors" and ready to be deployed to its final users - the students who are blind;

2. produces signals with higher perceptual salience;

3. molds to the palm of the hand;

4. is easier to maintain;

The first set of experiments showed us a correlation between how well the glove fitted and the participant's performance. This goodness-of-fit concept includes comfort and perceptual salience. By comfort we mean: freedom of hand and finger movements, the glove not being too tight or too loose on the hand. Var Erp [265] reminds us that comfort is an important issue on tactile displays because they require actual contact to the skin. This is specially true if the user wears the glove over longer periods of time (eg during a 50min class). By perceptual salience of the signals, we mean the cognitive effort one has to employ to perceive the direction the glove is conveying. A better "fit" makes this "signal deciphering" cheaper in terms of cognitive resources because it will enable stronger sensory evidence [230]. The fewer resources employed on this task, the more will be available to other concurrent tasks, like paying attention to the teacher. The signal detection theory teaches us that on way to increase sensitivity is to increase target salience. We therefore need a glove not only robust, but one that conveys strong and distinguishable signals.

In pursuit of a glove with those qualities, we decided to create a completely new model. The original models had too many actuators: 12, 16, and 20 for the round, square and rectangular models respectively. Assembling that many actuators within a glove was complicated and the actuators were inevitably be too close to each other. Fewer wires means more assembly room for work which leads to better wiring and robust gloves. Furthermore, we do not have 
perceptual salience between two actuators when they are too close to one another. We need to "spread" them over the palm of the hand. That is precisely what we did. Figure 2.12a shows a "map" of how the actuators should be placed on the palm of the hand. We now have only eight actuators placed as far as possible from each other. Each actuator conveys one and only one direction.

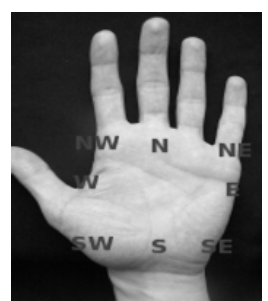

(a) The distribution of the motors on the palm of the hand.

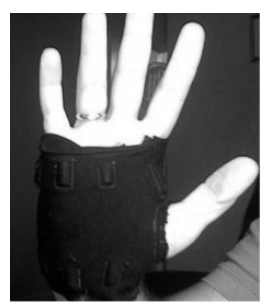

(b) The "Stretchable" third generation glove - left hand.

Figure 2.12: How the vibrating motors were arranged on the palm of the hand and the "Stretchable" glove.

Once the "map" was done, the challenge turned to be constructing such gloves. This model did not have straps. The user had to put his hand through the glove so that the actuators would be in maximum contact with the hand, increasing signal strength. Figure 2.12b shows what we called the stretchable glove.

We built six stretchable gloves: small, medium and large sizes for both left and right hands. We used this glove for several months and user's first reaction was that they could feel the directions better than any other previous model. However, when passing the hand through the glove's aperture, some users inadvertently stressed the wires. This was especially inconvenient for people with bigger hands - they normally broke the wires. We have gained on perceptual salience but lost on robustness. We needed a model that reconciles both features.

We needed another "generation" of gloves. Figure 2.13 shows the 4th-generation glove in three different views. The outer cloth layer is white to facilitate computer vision tracking. The raised line documents used in our experiments are also white. We use blue markers on the participant's fingernail. So, the camera sees more white, some skin color and the blue marker. Less colors and high contrast among them makes the tracking more stable and reliable.

One can see from 2.13a, we kept the straps in the new glove. They definitely help in making the glove comfortable and adjustable. 


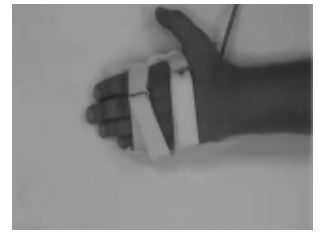

(a) The 4thgeneration glove viewed from the top.

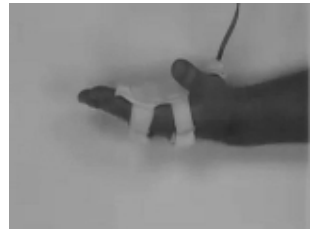

(b) The 4thgeneration glove viewed from the side.

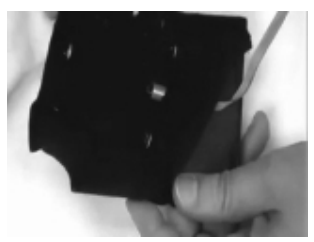

(e) The hard plastic provides extra protection to the wires.

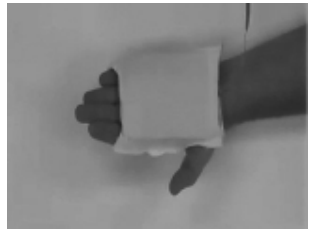

(c) The 4thgeneration glove viewed from the bottom.

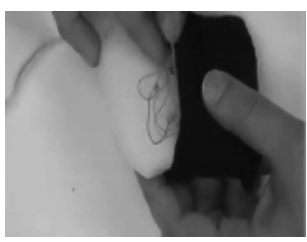

(f) The sponge provides both a better fit and protection to the wires

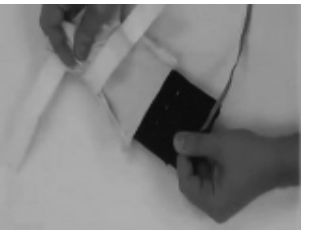

(d) The glove is built is layers.

Figure 2.13: The fourth and latest glove model.

The first real advantage of this glove is that we do not have to build as many models as we did for the Stretchable one. Right and left hand models are not necessary because the user puts the glove on the palm of his hand and not through it. She then adjusts the straps around her preferred hand. The straps help not only for controlling how tight the glove is but on the fine adjustment to the palm of the hand. This glove takes almost the entire palm of the hand (figure 2.13c). This is the result of our approach of spreading the actuators as far apart from each other as possible. This "spreading" of actuators was done because Cholewiak and Craig [42] reported improvement in signal detection when there is a larger separation between the points of the skin stimulated. We can see from the pictures, that the fingertips are free and some bending movement is possible.

The 4th-generation glove is built in layers to increase its robustness and help its maintenance (see figure 2.13d). The robustness is increased because all the pulling that takes places when the user is putting the glove on, is absorbed by the external layer. The internal layer, where the wires and motor connections reside, is protected from external manipulation as much as possible. For maintenance, all we have to do is remove the outer layer and access to the motors. The cylinder shown in figure $2.13 \mathrm{e}$ is a Cooper tube in which the vibrating motor resides. The copper tubes, containing the vibrating motors, are still kept within pockets. Also in figure 2.13e, we can see a piece of hard plastic coming out of the black cloth. This plastic has basically two functions. The first is to serve as an extra protection to the wires and their connections to the motors. The second is to provide a "hard portable surface". A surface to be used for a gentle increase on the pressure between the motors and the palm of 
the hand. The hard plastic, in conjunction with the straps are meant to control the pressure of the motors on the hand. In the last figure of this sequence, figure $2.13 \mathrm{f}$, we can see the sponge that goes inside that black cloth and the wires coming out of it. The sponge helps the glove to conform with the individual differences on the anatomy of the hand. As the user pulls the straps, the sponge helps the glove internal surface to mold to the palm of the hand. The motors are, obviously, mounted on the surface of the glove in contact with the hand. The wires that come out of the motors pass through the sponge and are soldered on the other side, protected by the hard plastic.

The vibrating motors have also evolved. The ones we ordered in the first stages of our work were also too fragile. Their external wires would come off at the slightest pull. That contributed a lot for the frustration early glove models caused. The new motors are more robust and definitely helped on the construction of the improved glove.

\subsubsection{Revisiting the signaling strategy}

The fourth generation glove worked so well that we decided to review the signaling patterns. Initially, we decided to always activate the motors at their highest vibration intensity. We wanted to maximize the perception of direction. And for that, we stopped conveying any indication of distance. Direction was the only component of the $D V$ being delivered to the haptic glove user. Using the fourth generation glove, we could put the motors to vibrate at half speed. Volunteers in the pilot tests of this new model could distinguish one speed from the other. With two speeds available, we can now use one to convey the idea that the PIF is "far" or "near". A detailed discussion on the navigation subsystem can be found in the next chapter.

\subsection{Transition}

In this chapter, we tried to demonstrate why our approach of sensory replacement is reasonable and why the haptic glove can be a good solution. After that, we described how we built our the glove and tried to justify our design and operational choices. We then laid out a series of usability questions that were answered by the experiments performed. The experiments, in turn, highlighted issues like comfort, robustness and perceptual salience. Those issues triggered another cycle of research and development, which gave rise to a succession of glove models. We imagine that we have finally reached a model that allies the fundamental qualities we think our glove needs. At this point in our project, we were confident that we could move forward to address issues of bi-directional discourse without fearing confounds from glove design issues.

The next logical step is to build the first functional version of the system to be used in classrooms, the $H D S$. We however, anticipate that the use of such system directly in such 
scenario would be a big jump. We have many things to be tested and adjusted before using it in a classroom. First, we need to test the system and fine tune it. Besides, this is not only about the system itself. It is also about the instruction materials. What should the teacher use? A poster, a projector, or a smartboard? Can the teacher write on the board? How will the student's version of the instructional material look like? What are its dimensions? These questions are related only with the system and instructional materials. However, we have deeper questions to be answered before moving on to the instruction experiment.

In the next chapter, we discuss and try to justify our decisions throughout the construction of the HDS. This will be the system we will use in our future experiments involving real mathematics instruction. It will be tested in different scenarios other than the instruction per se. It is so because we want to have all the issues we just raised addressed before the final experiment. Therefore in the following chapter, we show how the tracking of both teacher and student is done (discuss the actual algorithms), and what other technologies could have been used or still can be used. We also debate the limitations of our approach. 
Chapter 3

On to Discourse 



\subsection{Introduction}

Prior experiments establish efficacy of the device/system in passive interaction situations. However, success of complex combinations of perception using haptics for sensory replacement is not obvious [250]. Our approach requires students and instructors to engage in dynamic interactive discourse. Discourse engages a broad band of cognitive resources. Multimodal discourse requires tacit fusion of information while engaging in comprehension and discourse maintenance. More than discourse, learning is only possible through dialog between tutor and apprentince. In a dialog, there is social pressure to keep the conversation flowing. And the burden is heavier on the listener side Clark ([51] p 140), which in our case is normally the student. Wilson suggested that "when confronted with novel cognitive or perceptuo-motor problems, humans predictably fall apart under time pressure.", [296]. Support for fluent instructional discourse needs to be demonstrated. At this point, our research objectives were:

1. To test the interaction configuration of a "instructor" (or guide) pointing at a visual on the wall (along with the video tracking for this), with the student using the glove on a parallel graphic;

2. To observe if this new interaction enables more fluent and effective communication

3. To observe what new impediments will arise from this new form of communication.

4. To investigate what communication strategies will emerge from the guide/follower interaction in a setting similar to a real classroom.

In the following sections, we formally introduce our Haptic Deictic System - HDS. After that, we will review the literature related to computer mediated human to human interaction. We will see that, depending on the circumstances, technology can enable activities that would not be possible without it. We will also see cases where the system did not bring any benefits to the interaction because it did not properly accomodate the communicational demands of the task it was trying to enable. One common trait of all these works is their underlying theory: Clark's Common Ground [47]. Following such discussion, we present our phrase charade game, designed to test HDS on a deixis-laden dialog between a guide and blind follower. We also discuss the similarities between the guide/follower interaction in the charade and that of the instructor/student in a real classroom. After that, we explain the process of selecting the charades. We then discuss how the trials went. After that, we present our qualitative and quantitative observations. Finally, in the transition section, we talk about how this charade experiment influenced the course of this research and our response to the findings. 


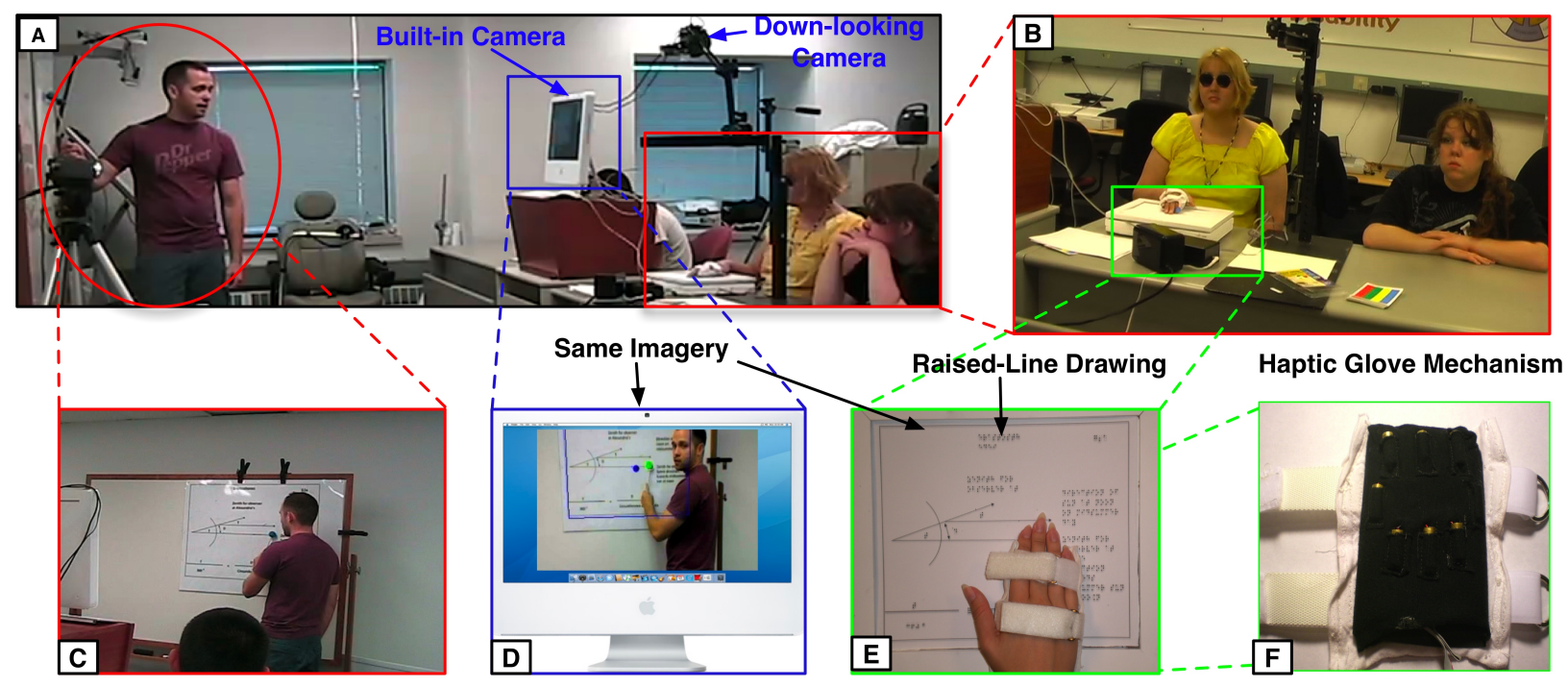

Figure 3.1: The Haptic Deictic System - HDS

\subsection{The Haptic Deictic System - HDS}

Figure 3.1 (A) (captured from the mathematics instruction experiments in college) demonstrates the classroom setup of our system. The instructor is pointing to a graphic and a pair of seated students (one SBVI and one sighted) are attending the lecture. The instructor's pointing gestures are tracked via the camera in the iMac. Figure 3.1 (B) shows the two SBVI reading the scaled raised-line version of the graphic on the poster. A down-looking camera tracks the student's reading finger (a frame of the down-looking camera video is shown in Figure 3.1 (E)). Figure 3.1 (F) shows the internal detail of the haptic glove worn by the SBVI. Figure 3.1 (D) shows the screen of the iMac in which a video of the instructor is shown with the reading location of the SBVI's TPA highlighted as a green dot.

During lecture, the built-in iMac camera tracks the instructor's pointing, and the downlooking camera tracks the current position of the reading finger. The system determines the disparity between where the instructor is pointing and where the students reading hand is positioned, and computes the current direction the student needs to move her hand to read the target location on the raised-line graphic and the vibrating actuator array in the haptic glove [204] activates in the appropriate pattern to guide the student to where the instructor is pointing, in essence providing the student with awareness of the instructors pointing behavior. Conversely, the iMac screen provides a feedback of SBVI reading behavior to the instructor, in effect, providing a form of 'gaze awareness' screen to give the instructor information of the state of attention of the student). 


\subsubsection{Vision Tracking Approach}

The overview shown in Figure 3.1 suggests several design and technology requirements to realize our HDS approach. First, the system must be able to relate and calibrate both spaces so that the system can determine the equivalent disparity of the reading hand over the raisedline graphic vs the pointing on the instructor's presentation graphic. This calibration must be self-updating because one cannot eliminate the possibility of the SBVI moving the raisedline drawing during reading. Second, the HDS is intended for use by individual SBVI in classrooms. This means that the tracking must be robust and low-cost (both monetarily and in computational load). Third, the tracking systems must function in real-time to provide timely coordination between the pointing, speech, and reading activity.

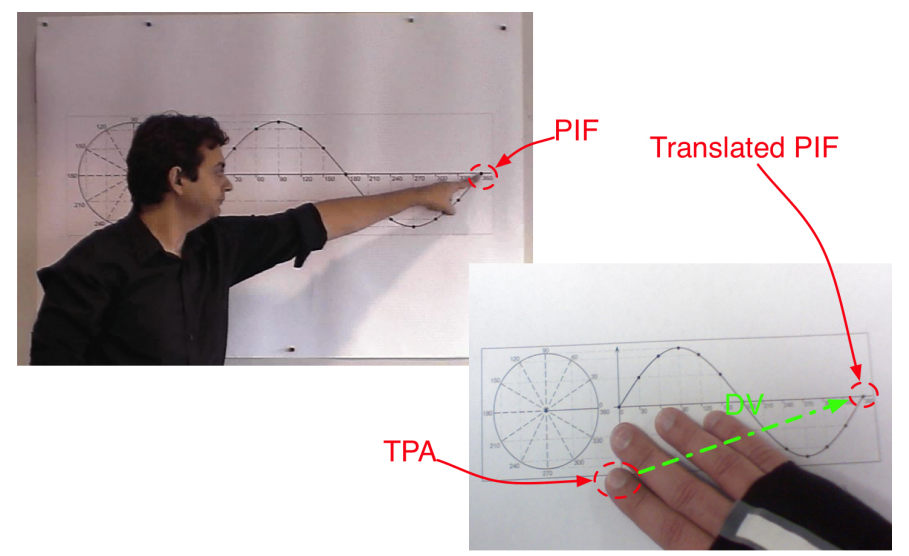

Figure 3.2: Points of Instructional Focus and SBVI Tactile Access

Figure 3.2 illustrates the signal provided to the SBVI. We define the Point of Instructional Focus, PIF as the location on the graphic pointed to by the instructor. The location where the SBVI is reading on her corresponding tactile image is the Tactile Point of Access, TPA. The signal that we communicate through the glove is the Disparity Vector, DV.

The system is comprised of six major components (described in the following sections). They are as follows:

1. Real-time tracking of the instructor's pointing. The objective of this component is to find the PIF.

2. Real-time tracking of the student's pointing. This component is responsible to find the TPA.

3. The translation of the PIF to the TPA's coordinate system. Instructor and student are tracked simultaneously by two different cameras: One observing the instructor and 
other the student. Each camera has its own coordinate system. We need to have both points on the same coordinate system to calculate the disparity vector (DV).

4. The haptic aided navigation subsystem. This is the part of the system responsible for delivering the DV to the student via the haptic glove.

5. The instructor's display. The instructor's display is a window in the system where the image captured by the camera looking at the instructor is displayed along with dots corresponding to both PIF and TPA. This display is updated in real time and can be used by instructor to follow the student's navigation to the PIF.

\section{Tracking the instructor's pointing}

For the instructor tracking, we use the background subtraction approach shown as figure 3.3. When the system starts, we ask the instructor not be at the board, to avoid her image being captured by the camera that will track her pointing. So, the initial frames the camera captures do not show the instructor (left figure). The system uses the information from the first several captured frames to calculate what will become the background image. To remove noise, the median value of the pixel from the first several frames are used after they are converted from color to gray.

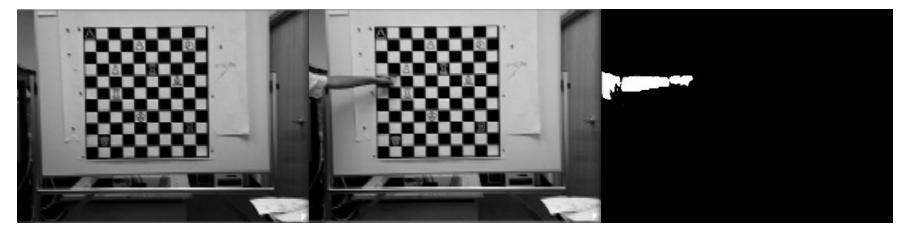

Figure 3.3: Background subtraction for PIF

Assuming the typical pointing morphology of a instructor's body, one can suppose that the extremal point of the body into the instruction graphic is the PIF. Given the background model previously extracted, we can extract the instructor's body from the graphic by a rapid background subtraction approach. Thus, interest pixel values on the instructor body (right figure) can be calculated. From this, the extremal point can be easily extracted as the PIF.

While this approach proved effective when the only activity performed by the instructor is pointing, it was not able to distinguish pointing from any other behavior when the instructor's hand was extended from the body (e.g., when she is writing on the board). A simple and robust solution to this problem is to provide the instructor with a 'pointing wand'. This allows her to signify a 'point' by simply moving it over the graphic. The design of this wand can be seen in figure 3.1 (D). To simplify processing, the wand is essentially a stick with a round colored disk at the end of it. For robust wand tracking, we employ a Gaussian color 
model in normalized $R G$ space, and use the Maximum Likelihood Estimation to find the best approximation for normalized $R G$. The color is comprised of a mean vector $\mu$ and a covariance matrix $\Sigma$. In Eq. 3.1, $x_{k}$ is a vector of normalized pixel values from the samples.

$$
\left\{\begin{array}{l}
\hat{\mu}=\frac{1}{n} \sum_{k=1}^{n} x_{k} \\
\hat{\Sigma}=\frac{1}{n} \sum_{k=1}^{n}\left(x_{k}-\hat{\mu}\right)\left(x_{k}-\hat{\mu}\right)^{T}
\end{array}\right.
$$

$x\left(x=\left(R_{n}, B_{n}\right)^{T}\right)$ corresponds to a pixel in normalized $R G$ space.

$$
R_{n}=\frac{R}{R+G+B}, B_{n}=\frac{B}{R+G+B}, G_{n}=\frac{G}{R+G+B}=1-R_{n}-b_{n}
$$

Basically, we need to build the color model of the wand disk before use in the instruction program. To built this model, we need samples of pixels of the wand. When the model is built $(\mu, \Sigma)$, we can classify each pixel, after background subtraction, by the model.

\section{The tracking of the student's pointing}

Tracking the student's reading finger can be tricky because we do not know a priori which finger a particular SBVI uses for reading (different individuals choose to use different fingers or even two fingers). Furthermore, there is always a need for anchoring. Anchoring occurs when blind readers leave one finger of the non-reading hand on a fixed position, normally at the beginning of the line or some distance trailing the reading hand to establish a reference. In this scenario, a camera observing the student's hand and fingers movements might not have the means to identify which finger is actually being used for reading. A simple solution is to ask the user which finger she uses for reading and to place a marker on the her fingernail. Hence, we can employ the same color extraction and tracking approach as for the instructor's wand.

\section{The translation of the TPA to the PIF's coordinate system}

Once we have found both PIF and PTA, the next step is to translate PTA's coordinates from the student's camera coordinate system to the instructor's camera coordinate system. As we discussed before, the system must constantly update the PIF to TPA translation because we cannot absolutely avoid the SBVI from bumping and perturbing her raised-line-drawing (to reduce this possibility, we actually provide a wooden frame with rubber cleats to hold the raised-line-drawing, but the SBVI can still inadvertently shift the frame). In figure 3.4a, the original PTA is shown in green and labeled $T P A_{o}$ for orginal. Similarly, figure $3.4 \mathrm{~b}$ shows the TP $A_{t}$ - TPA translated to the instructor's camera coordinate system. 


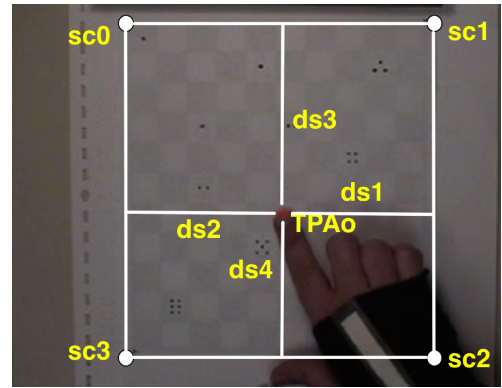

(a) Frame captured from the student's camera - its corners $(s c 0 \ldots s c 3)$, are known

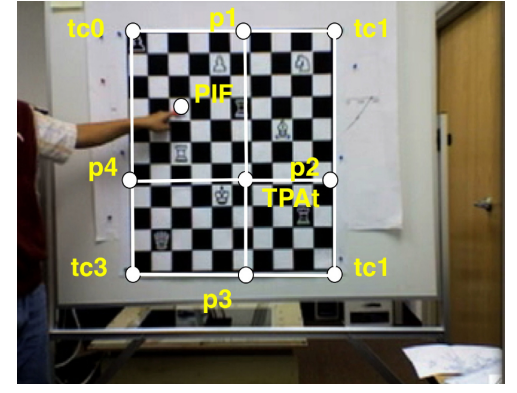

(b) Frame captured from the instructor's camera - its corners $(t c 0 \ldots t c 3)$, are known

Table 3.1: Translating PTA coordinates from the student's camera coordinates system to the instructor's camera coordinate system

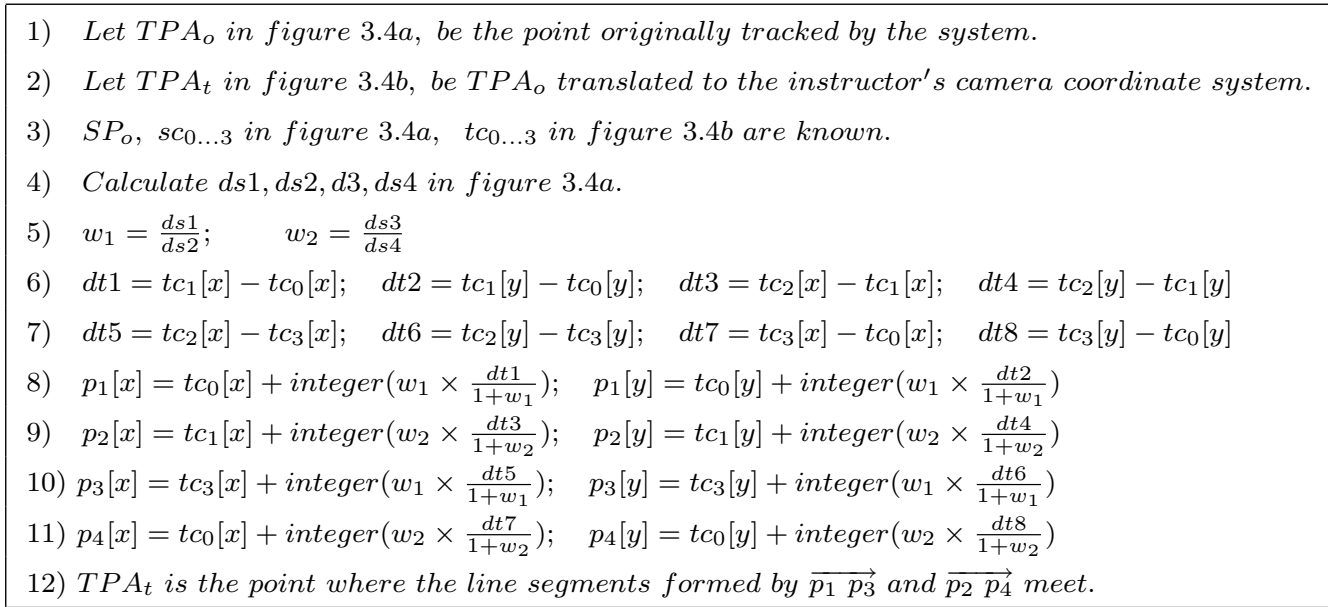

The algorithm presented on table 3.1 shows how from a set of known values, it is possible to do the translation.

\section{The calculation of the disparity Vector (DV)}

The final step is to calculate the Disparity Vector - DV - that goes from $P T A_{t}$ to $P I F$.

$$
D V=\left(\begin{array}{c}
\text { Direction } \\
\text { Distance }
\end{array}\right)
$$


Where

$$
\begin{gathered}
\text { direction }=\left(\frac{\arctan \frac{T P A_{t}(y)-P I F(y)}{P I F(x)-P T A_{t}(x)} \times 180^{\circ}}{\pi}\right)-90^{\circ} ; \\
\text { distance }=\sqrt{\left(T P A_{t}(y)-P I F(y)\right)^{2}+\left(T P A_{t}(x)-P I F(x)\right)^{2}}
\end{gathered}
$$

$D V$ is updated in real time as the cameras feed the system at a rate of 30 frames per second. The $D V$ is then sent to the student through the haptic glove. Note that there is a very tightly closed feedback loop on the system. As new $D V$ is computed, it is compared with the $D V$ computed just before it. The new $D V$ is delivered immediately replacing the directional signal being sent by the previous $D V$.

\subsubsection{The haptic aided navigation subsystem}

During the very first stages of this research, we studied how to use the different vibration intensities to convey useful information. Originally, we had 7 different levels of vibration intensity. As we pointed in the last chapter, we have given up using all these different vibration intensities in exchange for perceptual salience. We just set the motors to vibrate at the highest intensity to make the direction deciphering easier and therefore faster. It was a conservative move since the most important information to deliver is direction and not distance. However, the new glove models yielded better perception salience and encouraged us to review our original decision. So for the charade experiment, we had two levels of vibration intensity. The first speed is the one used in the previous experiments - the full speed. In the second speed, we have the motors spinning at half speed. This half speed is the furthermost speed from the first one we can get and still keep the vibration perceivable.

Therefore, in this version, we had two different speeds to convey distance. For that, we employed the driving a car metaphor - when you get closer to your destination, you slow down. Of course, we could have used the other way around - higher intensity when the destination is closer. Basically, we opted for the first alternative because when the target is further, the student can move her hand in a straight line to its vicinity. Once in the target vicinity, the student needs to proceed with finer movements. In this position, the student can easily overshoot the target with an ampler hand movement. If this happens, the glove will promptly change the direction being signaled. The new direction would be exactly the opposite of the old one. For instance, if the glove were sending north, and the student was close to the target and moved her hand too much to the north, her hand will suddenly turn to be at the north of the target. Then, the glove will start sending south instead of north. We have already noticed that this is confusing to people with less experience with the glove. So, we thought that vibration at intensity would induce the student to navigate slower, and be ready for sudden changes in direction. In summary, strong vibration signals that the target is far and while weak means that the target is near. 


\subsubsection{The Instructor's Display}

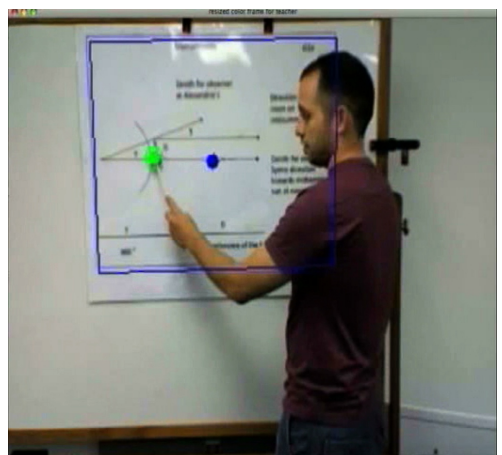

(a) Green Halo - TPA far from PIF, glove vibrating at full speed

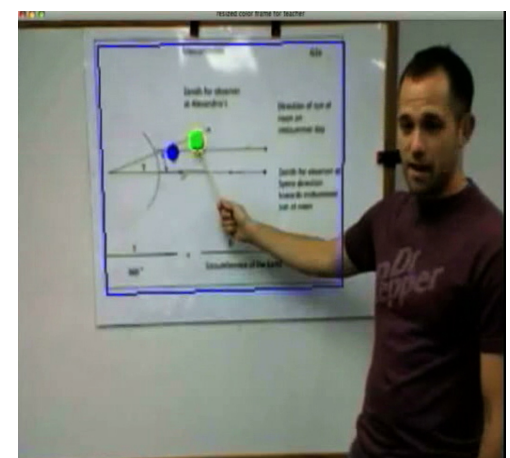

(b) Yellow Halo - TPA close from PIF, glove vibrating at half speed

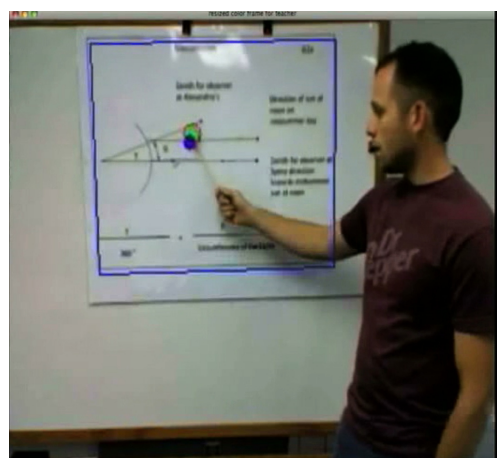

(c) Red Halo - TPA entering PIF area, glove not vibrating

Figure 3.4: Color codes for the instructor's display

The instructor's display, figures 3.4a, 3.4b, 3.4c, can be seen as an extra source of evidence of the SBVI behavior being presented to the instructor. The image that appears on that window is constructed from the frames captured from the cameras tracking both instructor and student. The green circle at the tip of the instructor's wand corresponds to the PIF, whereas the blue (dark colored) one is the TPAt. The blue rectangle is the instructor's gesturing space (TGS). All pointing done inside this area will be delivered to the student and conversely, any pointing done outside this area will not be delivered.

There is a halo around the dot corresponding to the PIF. This halo can have 3 different colors (green, yellow and red). The green halo (figure 3.4a signals the instructor that the student is still far from where he is pointing and the glove is vibrating at full speed. The yellow halo (figure 3.4b) indicates that the student is in the referent vicinity and because of that, the glove is vibrating at half speed. Finally, the red halo (figure 3.4c) is evidence that the student has entered the PIF zone and consequently, the glove is no longer vibrating.

\subsection{Common Ground}

Our intervention can be seen as a study of a particular "arena" of human-human technology mediated communication. Under this perspective, Herb Clark's [51] common ground theory has much to inform us. For Clark, "People don't want to just talk per se. They want to gossip, to complete a business transaction, to entertain each other, to solve a problem, to instruct and learn, etc" ([51] p XVII). For these activities to unfold, participants engage in both individual and collective atctions. The converstants must understand each other's 
intentions. Their actions, therefore, depend heavily on what they know that the other know: Their common ground. Each conversational turn increases the amount of the shared knowledge. This mutual knowledge accumulation through human interaction is what makes this theory interesting to us. At the end of a lecture, instructor and student should share the same knowledge of that particular lesson.

Monk [195] observed that common ground theory bridges two aspects of language: cognition and social interaction. For psycholinguistics, language production and comprehension of language are very cognitive. As for ethnomethodologists and sociologists, language is a social phenomenon. Clark's theory bridges the two fields and can make predictions for the design of electronic mediated communication.

At the heart of this theory is the principle of optimal design. Speakers design their utterances with the belief that the listeners will readily and uniquely understand what they mean. This belief is based on the current utterance and the common ground the conversants accumulated before. Perceptual and linguistic evidences along with community membership are Common Ground pillars [47]. They are as strong as their power of reducing ambiguity. Perceptual evidence is the strongest among them. It achieves its peak when the conversants are copresent and none of them have any perceptual disabilities. In that situation, the conversants can see and hear what each other is doing. Clark ( [52] p 92) asserts that "Everything we do is rooted in information we have about our surroundings, activities, perceptions, emotions, plans, interests. Everything we do jointly with others is also rooted in this information, but only in that part we think they share with us".

In a conversation, before moving on to the next contribution, participants need to have evidence that they were understood [48, 49, 55, 88, 89, 97]. If the speaker feels that the listener has not understood her, she will "repair, expand on, or replace the noun phrase in an iterative process until they reach a version they mutually accept. In doing so, they try to minimize their joint effort." [47]. This is the Least Joint effort principle. For Monk [195], "This effort can be measured in time, errors, and other resources necessary to accomplish the conversational objective". Deictic gestures play a very important role in lowering such effort. First because it establishes immediate physical copresence ([51] p 39) - simultaneously providing physical evidence to both speaker and listener about the object in discussion. Sometimes this "object of discussion" is a concept that took time and effort to become mutually understood. A simple pointing gesture at that concept can bring all that common ground back into the conversation. Second, because it is a strong mechanism for repairing from conversational breakdowns ([51] pp 48-52). If the listener does not understand what the speaker said, the speaker can point to the object of discussion and the listener will readily understand it. Third, because deictics add precision to the utterances ([51] pp 4852). Precision is important because it prevents communication errors. Communication errors demand repair which greatly increases the cost of communication.

We expect that people can see what we see [195]. When this is not true and we think it is, the likelihood of conversational breakdowns is very high. Repairing from such breakdowns 
seems to harder than they happen for any other reason [195]. This phenomenon has been observed before. Tatar et al. 261] reported their experiences with the Cognoter. Cognoter was a software tool for use in electronic meeting rooms developed in the 1980 at Xerox PARC (Palo Alto Research Center). The software was to emulate and enhance the function of a whiteboard through networked computers and a large-screen central display. Differently from a typical whiteboard, participants had to create discussion items on an private edit window. Items were then displayed to the others participants on the large screen. This feature made grounding hard to achieve because one conversant could make reference to a portion of the graph only visible to her. When that occurred, Deixis broke down.

Of course, the student who is blind cannot understand the instructor's pointing because she lacks a sense - vision. There, we have a channel breakdown, according to Clark's action ladder (table 3.2).

Table 3.2: Clark's Action Ladder

\begin{tabular}{lll} 
Level & Speaker A's part & Speaker B's part \\
\hline 4- Conversation & A is proposing a joint project $w$ to B & B is considering A's proposal \\
3- Intention & A is signaling that $p$ for B & B is recognizing that $p$ from A \\
2- Signal & A is presenting signal $s$ to B & B is identifying signal $s$ from A \\
1- Channel & A is executing behavior $t$ to B & B is attending to behavior \\
& & $t$ from A
\end{tabular}

In the action ladder, all levels start and end together (co-temporal actions), and each level is built on top of its lower level - Upward causality. Furthermore, if one level is complete, all levels below are also complete - Downward evidence [210]. Simply put, if there is a problem at the channel level, the "ladder" breaks down and all levels above it collapse. Listeners who are blind do not attend to the speaker when they gesture because of a channel problem lack of sight. To solve that problem, other channels (or modes) need to be explored. At the top of the ladder, the conversants fully understand the meaning of each other's actions and purposes.

We can tailor discourse according to the constraints imposed by the medium we are using, or to which grounding constraints [53] that medium affords. Examples of grounding constraints are: Visibility, Audibility, Contemporaneity, Simultaneity (see Clark [53] for a complete list and discussion). One example of such adaptation was observed by Otto: "We start and end telephone conversations in a different way from face to face conversations" [207]. Of course, each communication medium has communication costs associated with it. Some examples of costs are: Production, Formulation, Reception, Understanding, Delay, Asynchrony, Display, Delay, Repair ( refer to Clark [53] for more costs). Handwriting, for example, has a higher production cost than gesturing and speaking. Sometimes we breakdown complex utterances 
into simpler ones to lower its production, reception and understanding costs and to avoid repair [48]. Face to face communications afford cheaper display cost as one can point, nod or present an object [53. In our case, we expect to lower communicational costs by decreasing efforts related to: production, display, reception and understanding. We are, however, worried about delay costs. It is so because the student will have to navigate to where the instructor is pointing. We will return to this issue later on this chapter once we have discussed how delay costs can harm task performance.

The fact that face to face is the medium that enables the cheapest communication cost does not make it the preferred to all activities. If the goal is to refuse a request, letter is the more appropriate [82]. People choose to use phone over electronic chats when they need to be more precise in instructions [55]. When the task is to coordinate schedules, assign tasks or make progress reports, e-mail is the preferred medium [75]. The e-mail preference is interesting to discuss because despite the fact that it is an unimodal application which basically supports only text, participants do not get confused in e-mail discussions even when the subject is complex [70]. Maybe it is because grounding constraints like sequentiality, reviewability and revisability are more important for those tasks and are nicely implemented in e-mail. The point we are trying to make is that the task or the "joint activity", in Clark's terms, is the determinant factor on what grounding constraints a tool should enable and at what communicational costs.

We understand that face to face communication is still the preferable medium for teaching. On the first chapter, we argued about the importance of the close monitoring of the student's embodied behavior, as the instructors should be looking for signs of confusion, misunderstanding, for the students are entering their zone of proximal development ([276] $\mathrm{p}$ 84-91). Face to face is also the preferred medium for negotiating [8, 75] and reprimanding [82. Maybe the relationship between these two tasks and lecturing is the need to attend non-verbal displays that can give away what is in the listener's mind and heart. Sellen justifies the need for visual information in these scenarios as a way to assure "social presence" [240]. Obviously, as we posited before, face to face interaction that involves a participant that cannot see is quite different from its original concept. On chapter two, we have tried to show that the blind "see" through haptic exploration. Such exploration is slower and limited in comparison to sight. Therefore what the blind student "sees" becomes crucial. She does not see the instructor, she has access to what the instructor is pointing. Is this enough? What would be the communicational costs of this new way of interacting? Several researchers believe that face to face communication is the basis for theory and design of technology to support mediated communication [61, 162, 209, 285]. We start looking for answers in previous works that manipulated grounding constraints. We shall discuss now what these previous studies have to inform our project.

Kraut and colleagues [164] examined how video co-presence change the way people coordinate their conversation. In their experiment, participants repaired bicycles with or without the help of a remote expert. In the trials that had the help of the expert, the expert used either audio/video or just audio. Participants who were helped by the experts did a significantly 
better job than those who did not get support. Participants who were helped through both video/audio link did not perform significantly better than those who were helped only with expert using only audio. In no-video condition, the participants were more explicit, more detailed in their utterances. When they did not have video, both the participant and the expert had to encode in language the participant's state and the state of the task for timing of instructions to be effective. In this setting, we see no loss in task performance in the no-video condition. This is contradicted by Anderson et al 68] who report an increase in conversational turns for checking mutual understanding in tasks when only audio was available. Chapanis et al [39, 40] found no performance differences in cognitive tasks when the parties interacted through speech alone, face to face or video and audio combined. Reid 224 also found no improvement when video was added to audio. In fact, some researchers advocate that visual information may even detract from task objectives [240, 285, 290]. The reason for this might be the fact that the video being captured is normally from one's face and people spend only a fraction of time looking to each other's face [3]. When the discussion is around a certain object, gaze at others falls to $3 \%$ to $7 \%$ [7] and mutual gaze is even lower than that [3].

On the other hand, Venoit et al [270] showed that in a conversation involving a native English speaker and a non-native, the addition of video did help. The authors argue that due to the non-natives compensated their lack of fluency with gestures. Short et al [246] showed the absence of visual information harmed task performance when participants need to access emotional information. In our particular case, we have seen in chapter 1 that the novideo condition [219] decreases learning performance. We argue that this because if we take away from the dynamic combination of speech and imagery, we will be diminish concept transmission. So, we claim that having access to imagery embossed in the instructional material as the instructor lectures, is more important than the access to the her face. We shall now try to bring supporting evidence to such claim.

Several studies reveal that showing the workspace is more important than displaying the participants' faces when they are working remotely [21, 287, 289, 85, 111, 200, 285, 290, 163]. Whittaker and colleagues [289], for example, compared speech only with speech and shared workspaces for brainstorming, spatial design and collaborative editing tasks. The addition of shared workspace improved all task performances except for the brainstorming. The authors argue that this task demanded less spatial thinking. Kraut et al [162] arrived at the same conclusion. Other authors suggest that such improvement comes from increase of situational awareness [21, 150, 183]. Simply put, the participants are aware of the circumstances in which their and their partner's actions were performed. The action is happening on the workspace. This makes the communication cheaper [72].

The work of Churchill and colleagues [45] bears some resemblance to ours. They describe a tool, Anchored Conversations, designed to allow chat windows to be anchored to text documents. Two participants, not co-present, collaborate in the review of a text document. They both open a copy of the document on their computers. They use electronic chat to communicate. The difference is that the chat windows are anchored to the document 
section that is the object of discussion. So, when one participant scrolls down her version of the document, it also scrolls down her partner's copy. At that point, they can start a new chat window and discuss that particular section of the document. Because they were both on "the same page", they could be much more effective in their task of reviewing the document. The first similarity to our proposal is that the participants had their own version of the document, just like the instructor's poster and the student's raised line version. Second, when one participant decided to move to a new portion of her document, the system directed the partner to the same section but of her own document. Both copies of the document had the same format, one participant's actions were reflected into the other's workspace enabled the use of deictic references during their conversation and that greatly improved task performance. Other studies on remotely shared office documents corroborate with Churchill et al's work [176, 241, 288. Whittaker [286] concludes that shared workspaces are preferable because they promote situational awareness and shared perspective which give rise to deictic references.

An interesting example of the role deictic references play on improving task performance is brought by Clark and colleagues [54]. In their experiment, a director and a builder collaborate in building Lego models where the shared workspace was either visible to the parties or not. In the visible workspace condition, the partners exchanged, in average, less than half the words they did when the workspace was not visible. Interestingly, the average of words per turn was higher when the workspace was visible. The average time to complete the task was also less than half when the workspace was visible in comparison to invisible workspace. A great chunk of time in the invisible workspace condition was spent in checking (average of 7 seconds when the workspace was invisible, against 0.6 seconds per block when the workspace was visible). The authors also counted the number of turns where the participants used deictic expressions: "here, there, this (including these), that (and those), like this (and like these), and like that (and like those)". These expressions were found in $31 \%$ of the turns when the workspace was visible and only $11 \%$ when it was not. Furthermore, when the workspace was not visible, the number of errors were more than eight times higher of that when the workspace was visible. The authors conclude that when prevented from monitoring the workspace, speakers can compensate, usually at time cost.

Making the conversation shorter and more precise, should make it cheaper, not only with respect to the time to complete the task but also to the cognitive resources employed. More information can flow between the parties in less time and with less effort. This is a big change. Besides, the student will now have the chance not only to resolve the instructor's referents, but also to start making her own. Will they do that? If so, will they be aware that they did? What changes will the HDS bring to the student's discourse? As McNeill[190] observed, language and thought are linked. So we can expect changes on how the SBVI think. How will the HDS impact content uptake? We must acknowledge that we are still not in the position to answer such deep questions. We need first to be sure that we have the tools to enable such change and the means to collect evidence of that. This is the purpose of the study we are about to describe. 


\subsection{How the game is played}

The charade game is played in pairs: A sighted guide and a follower who is blind. The guide helps the follower to solve the charade by employing speech and deixis. The puzzle is presented on the guide's whiteboard as a rectangular grid of letters (Figure 3.5a), and on the follower's desktop as a concomitant braille grid (Figure 3.5b). Our haptic glove system was deployed to facilitate the multimodal dialogue. Words that make up a 'clue phrase' are hidden in the grid as horizontal, vertical, or diagonal letter chains that can run in any direction. The cognitive activity of the discourse dynamic is represented by a 'catch phrase' that the followers have to discover from the clue phrase. For instance, in one puzzle, the clue phrase is "Blink blink small sun," and the associated familiar catch phrase is "Twinkle twinkle little star". The guide is aware of the catch phrase, but cannot tell the follower the answer directly.

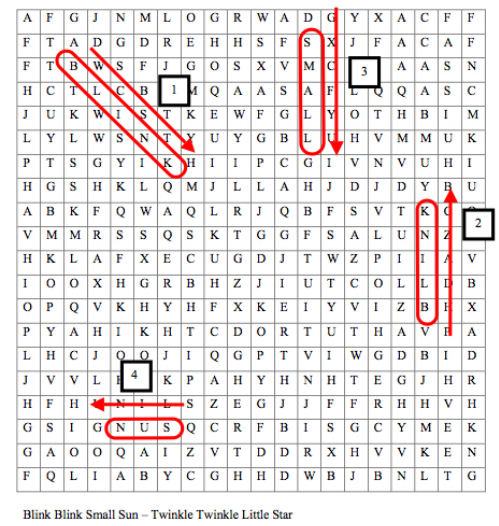

(a) The guide's poster

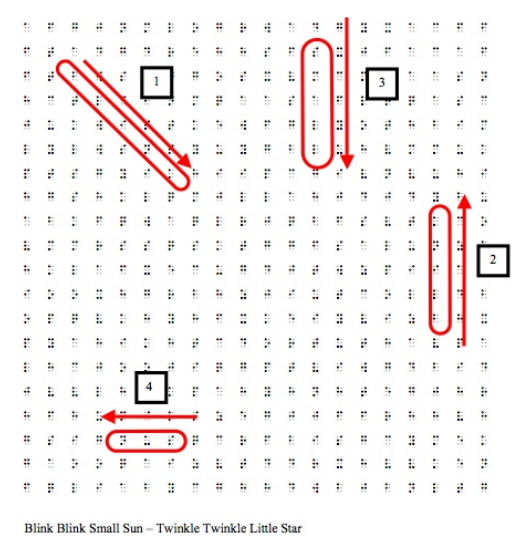

(b) Follower's braille version

Figure 3.5: Puzzles containing the clue phrases

We took care to ensure that the catch phrases are familiar to the anticipated student participants. We began with a list of 50 catch phrases from a phrase book. We narrowed this to a list of 12 most generally familiar four-word-long phrases using a survey of college students and staff members in our department. Since our anticipated subject pool are American-born, we used only native English speakers to judge the familiarity of the catch phrases. Of the 12 phrases selected, we used only the eight phrases judged most familiar. These phrases along with their clue phrases are tabulated in Table 4.14 and a complete listing of the survey's results can be found on page 191. In our post-study interview, we determined if the catch phrases used were known to the participants prior to the study. Trials using unfamiliar catch phrases were removed from our analysis. 
Table 3.3: Catch and Clue phrase pairs

\begin{tabular}{|c|c|c|}
\hline Rank & Catch Phrase & Clue Phrase \\
\hline 1 & Twinkle twinkle little star & Blink blink small sun \\
\hline 2 & Monkey see monkey do & Gorilla look gorilla execute \\
\hline 3 & My lips are sealed & Me mouth be shut \\
\hline 4 & Never a dull moment & Nevermore one dumb instant \\
\hline 5 & Out of this world & In the next galaxy \\
\hline 6 & See you later alligator & In a while crocodile \\
\hline 7 & Don't hold your breath & No stop thy respiration \\
\hline 8 & One for the road & Uno to a route \\
\hline
\end{tabular}

\subsubsection{Similarities between the charade game and instruction}

In the figures above, one can see that the words are written in different directions. They are written in a descending left to right diagonal as the word "Blink", or in backwards as "sun". We borrowed this from the traditional word finding game. It can be seen as an important precursor of the mathematics graph and charts that will be used in the future instruction experiments. When lecturing, instructors can address graphs in a variety of ways, depending on the situation or on their instructional objectives. This random writing direction in the charade is a way of trying not to make the pointing too predictable for the follower, although we must acknowledge that a word can be written in only one of the eight directions.

In word puzzles, letters go in one direction to form a bigger chunk of information - a word. During the charade experiment, the guide, pointing at the "S" in the word sun, says: "... it starts here and goes in this direction ...". Similarly, one can imagine a situation where the instructor is presenting a sine wave (figure 3.6 ). She can say: "... It is a perfect wave ... it goes diagonally up, in a curved way ...\{points at "A", "B", "C" $\}$. " The hand movement is slowly revealing the form of the curve, just as the letters put one after other reveals the word. This kind of discovery strategy is precisely the one those who are blind use to perceive the form of the things real world: through sequential tactile exploration. Spatial imagery processing ability does not depend on visual perceptual experience: The muscular movements the student does when she explores the shape of an figure embossed on her document might enable the formation of the mental image of that figure [131, 179, 137]. The idea that mental image can be produced by muscular discharges was first developed by W. James in the chapter on "Imagination" in his "Principles of Psychology" [132] in the 19th century.

Another important resemblance is the fact that the guide, aware of the solution, leads the student through a discovery process. In this sense, the dialog in the charade experiment is similar to the one expected in real instruction. This offers us an unique testing opportunity. We can assess: 1) How the system works; 2) How the system works together with raised 


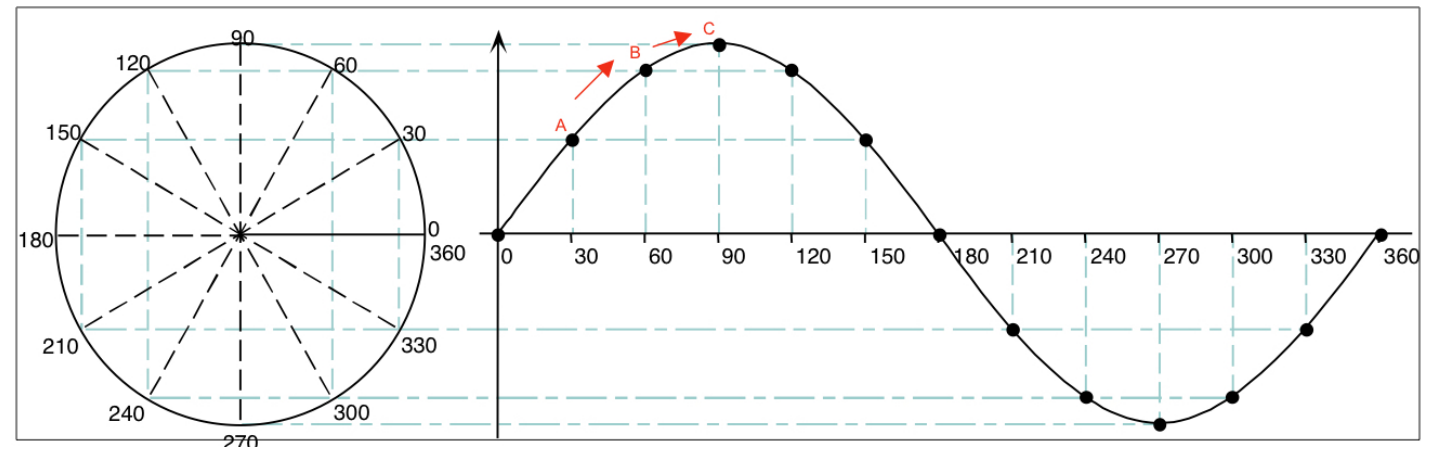

Figure 3.6: Introducing a sine wave

lined documents; 3) What are the interactional strategies guide and follower will develop as they explore the new affordances the system brings. This is a good opportunity to identify the impediments that will arise from system use.

\subsection{Recruiting Participants Who are Blind}

We conducted our studies at Wright State University (WSU) where there is a concentration of students with a wide range of disabilities and where these students are integrated into the general student population. This environment is somewhat unique because of the low percentage of students with disabilities who attend college. Only a very small number of high school graduates who are blind go to college [252]. Since our eventual goal is to conduct experiments on mathematics instruction in the form of short multi-day courses, we concentrated our recruitment on undergraduate students with similar mathematics exposure. We also wanted to be able to compare our results from the Phrase Charade with the performance of the same students in the mathematics instruction.

\subsection{The trials}

We had four followers (denoted G, N, M, R) and three guides (denoted DO, DA, J) for this experiment. There were two male and two female followers; and two male and one female guides. All participants were WSU students, followers undergraduate and guides, graduate students. Once these initial tasks were taken care of, we introduces the student the glove. First, we had them say a direction (i.e. east, northwest) and we would have the glove vibrate in the indicated direction. Next, we would give a random direction and have the student tell us which direction they felt the vibrations. After this initial time of acclimation we would start the first charade. 
Our first trial was with $\mathrm{D}$ as the guide and $\mathrm{R}$ as follower. R was wearing a "third generation" glove (we build four generations of gloves) and it was too tight on his hand. He was able to distinguish the glove signals but he struggled to effectively follow the glove's directions. $\mathrm{R}$ did not make it through the three charades in one session. Later, we were able to make minor adjustments before $\mathrm{R}$ returned to finish the experiment. This made for a better, second session and ultimately led to the development of another glove, which would be better suited for R's hand.

On the second trial day, we first had the pair DA (guide) and $\mathrm{N}$ (follower). N used the "remediated" 3rd-generation glove. This trial went surprisingly much smoother than the first one. $\mathrm{N}$ was calm and confident on the signals she was receiving from the glove. In the following two trials we employed the new 4th-generation glove. Both $\mathrm{M}$ and $\mathrm{G}$ used this new model. Owing to developmental deformities, M's hands are as small as young child's. Because of that, even using the small glove, $M$ had difficulties deciphering the directional signals. The glove had a pretty good fit to G's hand and no problems directly linked to the glove were observed.

The trials confirmed one of the findings we had on the initial studies: User performance is closely related how well the glove fits on the user's hand. A new challenge might have been uncovered: the participants multiple disabilities. M's multiple disabilities placed a more challenging obstacle for her interaction. If she wants to continue helping us, we will be forced to build her a special extra-small glove, which is also an engineering challenge. For now, we see $\mathrm{M}$ is an outlier among our target population.

As for the guides, they all were very excited about this technology and rapidly understood how use the system and to play the game. DO had a hard time refraining from giving directional clues to her followers. In the middle of the experiments we had to reaffirm that he was not supposed to give direct indicators of direction. DA and J played their role flawlessly. They all heavily relied on the instructor's display to: 1) To track where the follower was; 2) To adjust his/her discourse accordingly; 3) To build trust on the system. We shall now take a closer look at the points we have enumerated.

The use of the display for tracking where the followers' were at a given moment was obvious and for that purpose the system worked with no flaws. In fact, it worked so well that the guides almost didn't look directly at the followers. Their gaze was most of the time directed at the display. This could be an indicative that the system could be used in distance teaching/learning. During the debriefing session, DA recognized that she had paid more attention to followers themselves and less to the display. We observe that the mere introduction of the instructor's display brings big change on the guide/follower interaction and could raise a new set of research questions.

As predicted, the guides waited for the follower to resolve the referent before moving on to the their next discourse contribution. However, they did more than that. They encouraged the followers during their navigation to the referent, saying things like: "you're almost there", "there you go". We believe that this was a strategy to alleviate the follower's pressure and 
increase their social presence. Furthermore, the guides showed signs of happiness when the followers finally picked the referent - just as if they were a team that has just accomplished their mission. Maybe the guides knew that the only big challenge was to get to the referent. Once the follower resolved it, everything else should be easy and straightforward. The guides cheered enthusiastically when the followers solved the charade. This was so evident that, throughout the trials, we needed to reinforce that we were assessing the system, not the follower's performance. We believe that during the instruction experiment, the participants will behave as there will be not such an explicit moment of team achievement.

The third major observation on the participants' behavior was their inspector-like manners. This initial investigative stage was also observed by Williams et al [294]. The authors described that the user's first learned how to "control" the system and then tried to "read or interpret" its responses. In our case, the guides tried pointing with all fingers extended and then with only the index finger. They also tested the system with laser pointers, pen and other elongated object they could find. They were trying to see what the system can track. They also tried pointing with one hand and then with the other, alternating their body posture before the camera. Williams and colleagues called this phase intrinsic interaction. This behavior was observed both during the training phase, which was held before the charade, and during the charade per se. We suppose that they were trying to understand how the system responds to their actions. In the next phase, the instrumental interaction, Williams et al saw participants trying to interact with each other through the system. It seems that our participants went through the same phases. One important difference is that in our case, the experimental procedure - solving the charade - forced the interaction between guide and follower, whereas in Williams' work, it appeared naturally.

\subsection{Guides' and Followers' Experiences}

After the trials, we asked the followers 23 questions and 19 to the guides. The questions took the form of a set of statements to which the participants responded on an agreement Likert scale (1-strongly disagree to 5-strongly agree, 3 being no opinion). A complete listing of the follower's questionnaire is shown on page 196, whereas their answers are on page 204. The guide's questionnaire can be found on page 208 and the respective answers on page 215. We shall now discuss how they perceived the experience.

All but one follower agreed that the conversation flowed naturally. They all strongly agreed that they can perform better with practice, and that they are willing to participate in future experiments because they believe this technology will help the SBVI. All but one follower said they performed better with the system than having someone physically holding their hand. They also unanimously agreed (or strongly agreed) that having access to where the guide was point improved the conversation and that, because of the system, they were better understood. All but one follower reported that they could read the letters while talking to the guide and that using the system did not interfere on their thinking of the charade solution. 
Three of the four participants agreed that they would have asked more clarification questions had they not used the system. All followers reported that using the system made them pay more attention to the guide than they normally do in a regular class and that the system gave them a better understanding of what the guide was trying to say.

As for the guides, they all agreed (or strongly agreed) that: The follower could easily access where they were pointing to, the conversation flowed smoothly, they used pointing to clarify themselves and avoid misunderstandings, they were able to speak less and be more precise, the followers pointing was precise (all strongly agreed), the instructor's display helped them to match their speech to the followers pace, the instructor's display helped them to understand the followers behavior, the system will be useful in real world teaching, they asked fewer confirmation questions than expected (all strongly agreed), the system helped them in perceiving the followers intentions.

We will now discuss the comments made by the participants as they answered the questions. Among the followers, $\mathrm{N}$ commented that she would feel very comfortable with the glove if she had a chance to practice more. She also noticed that if she pressed the motors towards her palm as in a "squeezing" movement, the motors would stop spinning. The constant changes in directions, when approaching the target, frustrated N said: "I was not able to find the right spot". This issue appeared in all trials. None of the participants noticed the change in the vibration intensity when they approached the target. $\mathrm{N}$ also affirmed that she didn't find herself pointing and asking questions. Both M and G didn't make any comments to the questions - only straight answers. The final question was actually a request for suggestions to improve the system. M commented that "there were too much vibration" and that "the system would be useful actors and dancers". G suggested that we should "Widen the parameters for target zone". R said that he had difficulty navigating and paying attention to the guide. As for possible improvements, R suggested the glove should convey only four directions instead of eight. He also perceived that the system was tracking the guide's every movement and there should be a way of filtering out the unwanted movements because they can confound the follower.

The guides left the experiments with the conviction that the system works but there is room from improvement. It was also clear that some trials went better than the others. We debate strategy differences that might have led to better performance in the next section. DO suggested the use of projectors instead of posters and along with laser pointers.

The follower's comments seem to contradict the positive answers they gave. A clearer picture of what really happened should arise from the data analysis discussion we will have in the next section. For now, it appears that all participants (followers and guides) were excited about the technology potential and but the trials did not go as well as they expected. 


\subsection{Data Analysis}

All trails were videotaped from three different cameras capturing different aspects of the interaction. We wanted to investigate how the pairs worked as teams and what strategies they have developed to accomplish their common goal. Furthermore, we wanted to gain insight from their embodied behavior, looking for signs of frustration, confusion, happiness, etc. Beyond that, we wanted to analyze the fluency of the dialog; how many turns were necessary for the follower to assemble the clue phrase; if they ever used deictic words, like: "here", "there", "this", "that". Figures 3.7a and 3.7b show how the cameras were arranged and a frame captured by camera number 3 .

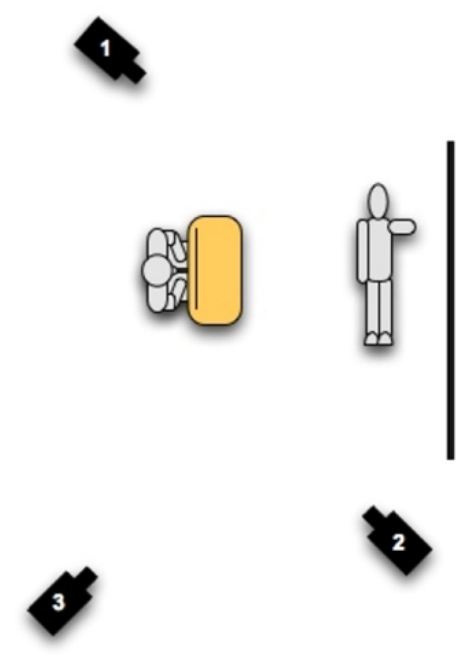

(a) 3 cameras to capture different aspects of the conversation

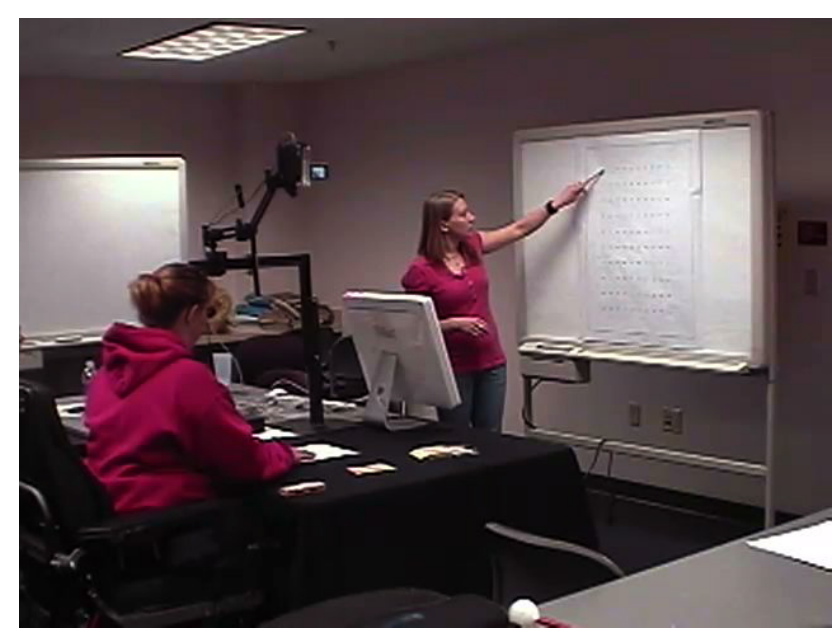

(b) Picture taken from camera 3 in an actual trial.

Figure 3.7: Cameras to record the trials from different perspectives

Camera 1 has close up look at the guide. This camera also captures the instructor's display and it is possible to observe when the guide gazed at the monitor.

Camera 2 closely observes the follower. The camera also sees the brailled document on top of her desk. Such vantage point allows us to observe signs of difficulty in reading the thermo-formed document. It also enables us to identify what kinds of problems arise from the haptic aided navigation.

Camera 3 sees the whole scene. From this angle, it is better to observe how the parts react to each other's behavior. 
All guide/subject pairs were able to complete the charade. We need to know is what were the communicational costs and what strategies were developed, which ones were more efficient and why. We acknowledge the small number of trials at the same time we posit that even with few numbers we could observe interesting trends. We shall now present and discuss the data.

Table 3.4 shows a summary of the trials. In the column trial, $N 3$ for example, means the third charade N solved. N was presented the clue phrase Gorilla look gorilla execute whose solution is the clue phrase Monkey see monkey do. N took 336.14 seconds (sec) from the presentation of the first letter of the clue phrase, $G$, to when she uttered the catch phrase. Of the $336.15 \mathrm{sec}$, she took $312.14 \mathrm{sec}$ to verbalize the clue-phrase, marking the end of the letter finding and letter/ word concatenating process. The final $23.99 \mathrm{sec}$ was spent in conversation with the guide about the possible solutions. Still in table 3.4 , the trails are sorted in descending order within each catch phrase block. For example, N3 is the first trial within the Monkey see monkey do charade because she reached the solution within the least amount of time. Notice that we do not have the trial M3. M took so long to complete her M1 and M2 trials that there was no time left for M3. We shall now discuss the data presented on this table.

Table 3.4: Summary of the trials - Times in seconds

\begin{tabular}{|c|c|c|c|c|c|}
\hline Catch phrase & Clue phrase & Trial & $\begin{array}{l}\text { Time to } \\
\text { Complete } \\
\text { charade }\end{array}$ & $\begin{array}{c}\text { Time to get } \\
\text { clue to } \\
\text { phrase }\end{array}$ & $\begin{array}{c}\text { Time to } \\
\text { map clue } \\
\text { phrase to } \\
\text { catch phrase }\end{array}$ \\
\hline \multirow[t]{4}{*}{ Monkey see monkey do } & \multirow[t]{4}{*}{ Gorilla look gorilla execute } & N3 & 336.14 & 312.14 & 23.99 \\
\hline & & G2 & 396.00 & 391.00 & 5.00 \\
\hline & & R3 & 626.00 & 611.00 & 15.00 \\
\hline & & M1 & 831.00 & 801.00 & 9.00 \\
\hline \multirow[t]{3}{*}{ My lips are sealed } & \multirow[t]{3}{*}{ Me mouth is shut } & $\mathrm{N} 2$ & 314.85 & 216.99 & 97.86 \\
\hline & & $\mathrm{R} 2$ & 433.00 & 342.00 & 91.00 \\
\hline & & G3 & 412.50 & 350.00 & 62.50 \\
\hline \multirow[t]{4}{*}{ Twinkle twikle little star } & \multirow[t]{4}{*}{ Blink blink small sun } & N1 & 323.50 & 229.50 & 93.00 \\
\hline & & G1 & 548.30 & 544.30 & 4.00 \\
\hline & & $\mathrm{R} 1$ & 605.00 & 605.00 & 0.00 \\
\hline & & M2 & 864.00 & 789.00 & 75.00 \\
\hline
\end{tabular}

The first thing that calls our attention is that $\mathrm{N}$ was the fastest in all charades. Her advantage came exclusively from the first part of the task - acquiring the clue phrase. She was the slowest mapping the clue phrase to the catch phrase. However, her advantage in the first part was so great that she still got the fastest overall time. Why was that? One possible explanation is that she is simply faster deciphering the directional signals. We, however, did 


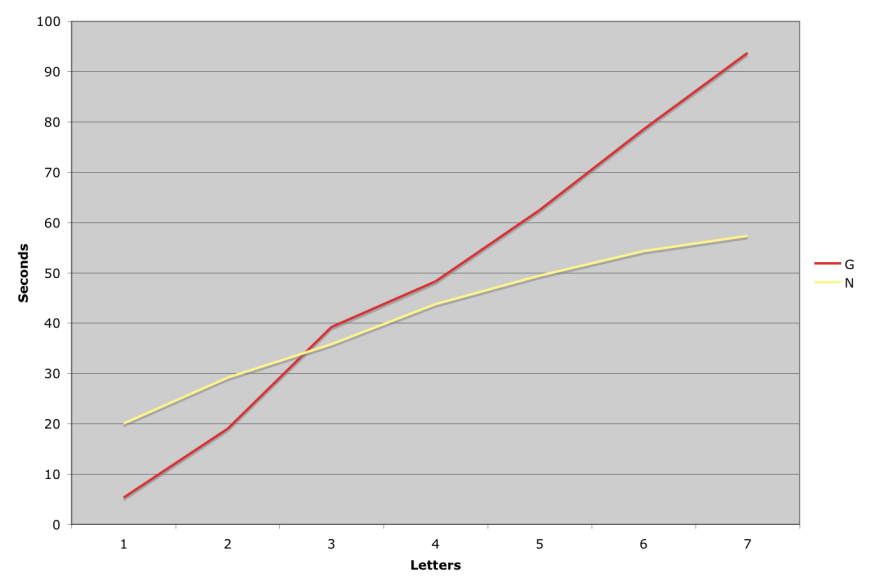

Figure 3.8: Time to find the letters of the word Gorilla

a very close comparison of her behavior with that of G's. For that, we used MacVissta [227], a system for multimodal analysis. With that tool, we were able to annotate what every participant said, at what time and what they were doing at the time of their utterance. There was no important difference in the times to get to the first letter of each word. This is relevant because getting to the first letter of the word implies in longer distances to travel. Once the subject has found the first letter, the remaining letters are neighbors - therefore closer. The difference, though, was found when making the transition from one letter to the next. Figure 3.8 shows the times both participants took to find the letters of the word Gorilla. We can observe from the graph that $\mathrm{G}$ travels in an approximately constant speed while $\mathrm{N}$ accelerates from letter to letter.

Another interesting observation can be found on the behavior of the guides. After being briefed, guides were pretty much free to engage on any strategy they wanted. At the beginning of each word, N's guide (DA) strongly reinforced the direction of the chain of letters that formed the word. Once $\mathrm{N}$ found the first letter of the word, DA would say: It is a 5 letter word and it goes in this direction. Then, she moved her hand in the appropriate direction and made sure that $\mathrm{N}$ followed her move. This strategy assured that $\mathrm{N}$ knew, in advance, the number and direction of the letters. G's guide just went letter by letter. N's guide (DA) also guided R, before N's trial. However, DA/R did not develop the described strategy and the pair's performance was similar to that of $\mathrm{G}$ and her guide.

Back to DA/N's trial, it seemed that N employed less effort than everyone else. She was able to concatenate letters and words as they were found. For example, she was pointed to the letter B and she uttered: "B" then she was directed to the letter "L" and instead of saying: "L", she said: "BL". She also used the same strategy with the words. At the last word of the clue phrase, she would say the entire clue phrase, without being asked to. Guides needed to reinforce the other followers' memory throughout their trials. 
DA/N strategy also led to a more economic dialog. Many times, DA would just point to the next letter, saying nothing. This action was interpreted by $\mathrm{N}$ as continuer, to use Clark's term ([51] pp 114, 150). Table 3.5 shows how many discourse turns each pair took to solve the charades.

Table 3.5: Discourse turns to complete the charades

\begin{tabular}{|c|c|r|}
\hline Catch Phrase & Guide/Follower & Discourse Turns \\
\hline Monkey see monkey do & $\mathrm{DA} / \mathrm{N}$ & 103 \\
& $\mathrm{D} / \mathrm{G}$ & 167 \\
\hline My lips are sealed & $\mathrm{DA} / \mathrm{N}$ & 91 \\
& $\mathrm{D} / \mathrm{G}$ & 134 \\
\hline Twinkle twinkle little star & $\mathrm{DA} / \mathrm{N}$ & 99 \\
& $\mathrm{D} / \mathrm{G}$ & 192 \\
\hline
\end{tabular}

One might argue that guide's personal style plays a very important role on the interaction - one person can simply be more talkative than other. Despite differences in style, it is important to better understand the impact of the system in what the parties were talking about. For instance, participants should not be discussing on how to get to the letter being pointed because this is the role of the system. We coded the conversational turns according to a method suggested by McNeill [191]. In such method, conversational turns can be assigned as pertaining to either one of the following referential threads: Object level, Meta level and Para level. In object level thread, conversants are discussing about their joint project [195], in our case, taking direct actions to solve the charade. In meta level, the subject is the discourse itself. Turns related to discourse repair were also coded as meta level. Para level utterances relate to the conversants' individual experience. Words of encouragement like: "There you go", "take your time", etc were coded as belonging to the para level thread. Low percentage of object level turns suggests lack of focus on the task objective. Table 3.6] shows the percentages of object level turns in each trial.

The numbers show that in about one third of the turns, the parties were talking about something other than their objective. These values are not good If we imagine the same task being performed by a guide/sighted follower pair. The results suggest that interaction did not go as smooth as we anticipated and this issue should be addressed.

We also coded for what we called the task phase. Normally to arrive at the charade solution, the pair would need to pass through three distinct phases. The first one, is the letter presentation or simply $L$, when the guide presents the follower the letter and the follower shows evidence of acceptance. If the pointing was enough for the follower to resolve the referent, the pair would either move to the next letter presentation or to a new phase - the 
Table 3.6: Percentage of object conversational turns

\begin{tabular}{|c|c|r|}
\hline Participant & Catch Phrase & \% of object level turns \\
\hline $\mathbf{N}$ & Twinkle twinkle little star & $66.36 \%$ \\
& My lips are sealed & $68.83 \%$ \\
& Monkey see monkey do & $61.82 \%$ \\
\hline $\mathbf{R}$ & Twinkle twinkle little star & $55.56 \%$ \\
& My lips are sealed & $64.61 \%$ \\
& Monkey see monkey do & $59.52 \%$ \\
\hline $\mathbf{G}$ & Twinkle twinkle little star & $42.33 \%$ \\
& Monkey see monkey do & $65.24 \%$ \\
& My lips are sealed & $65.17 \%$ \\
\hline $\mathbf{M}$ & Twinkle twinkle little star & $52.60 \%$ \\
& Monkey see monkey do & $60.10 \%$ \\
& My lips are sealed & $62.30 \%$ \\
\hline
\end{tabular}

concatenation phase, or $C$. The $C$ phase can occur when the pair is concatenating either letters to form words, or words to form the clue phrase. It might have been the case when pointing was not enough, and the guide was force to give more information to the follower. In this case, the pair engaged in the expansion phase, or E. Another possible situation is that when the follower is completely lost in the navigation or on the game per se. In this case, the guide helped recovering ground. We call this phase, the repair phase. All phases described so far are in fact sub-phases, of the larger acquiring the clue phrase or $A$ phase we presented earlier. All other activities the pair performed after that were coded as negotiating the solution or simply $S$. Previous works proposed different coding schemes for the different roles the participants play in the joint activity [54, 121, 262]. We, however, decided to treat the pair as a unit because one part cannot proceed without the other. We think that chopping down the trials into phases provides the opportunity to make comparisons against the expected behavior for sighted students under the same discourse content. If the follower could see, the $A$ phase would be completed much earlier. We do not believe that there would be important differences during the $S$ phase. Within the $A$ phase, the $L$ sub-phase, would be over earlier and there would be almost no need for the part to engages in $E$. So, $E$ phase would be the point where a trial involving a sighted follower would mostly differ from the ones we had with our blind participants. Sighted followers would almost immediately resolve the referent as it takes only a quarter of a second for the first human eye saccade at a peripheral target [215]. Therefore, we understand that this is the crucial phase: Longer $E$ phases show that the participants had difficulties resolving the referent.

Table 3.7 shows both the number of expansion turns, the time the pairs spent with them and the percentage of expansion time over the total time spent in acquiring the clue phrase. 
Table 3.7: Expansion turns - times in seconds

\begin{tabular}{|c|c|c|c|c|c|}
\hline Catch Phrase & Trial & Turns & $\begin{array}{c}\text { Time spent } \\
\text { in } \boldsymbol{E} \text { phase }\end{array}$ & $\begin{array}{c}\text { Time spent } \\
\text { in } \boldsymbol{A} \text { phase }\end{array}$ & $\begin{array}{c}\boldsymbol{E} \text { time } \\
\text { over } \boldsymbol{A} \text { time }\end{array}$ \\
\hline \hline Monkey see monkey do & G2 & 48 & 179.02 & 391.00 & $45.79 \%$ \\
& N3 & 23 & 139.96 & 321.14 & $43.58 \%$ \\
\hline My lips are sealed & G3 & 18 & 87.40 & 350.00 & $24.97 \%$ \\
& N2 & 12 & 78.51 & 216.99 & $36.18 \%$ \\
\hline Twinkle twikle little star & G1 & 70 & 222.55 & 544.30 & $43.89 \%$ \\
& N1 & 15 & 58.15 & 229.50 & $23.33 \%$ \\
\hline
\end{tabular}

Unfortunately, the great disparity in the numbers does not give us much room for analysis. The greatest difference is on the Twinkle twinkle little star charade. There, DA/N were much faster than $\mathrm{DO} / \mathrm{G}$ and this difference is reflected on the expansion turns and their respective times. On the trials where the $A$ time difference is not great, the $E$ time is also not that significant.

It is worth noting though, that in the worst case, the percentage of time consumed by expansion (45.9\%), might be close to what is considered by common knowledge in the area. As we pointed earlier, according to Dick [64], it takes 1.5 longer for a student who is blind to cover the same instructional material than that of a sighted counterpart. We do not want to make a closer comparison between the two numbers because this might be premature. However, we should "keep an eye" on Dick's number because one of the pillars of our research is precisely communication economics.

In Clark's ([51] pp 126-127) original proposal, we can engage in expansions at any moment of the communication and that is not related to any particular task. It is inherent to the communication process. We confined the concept use to acquisition of the clue phrase phase for the reasons discussed above. We, however, kept its essence: the need for extra information on the part of the listener.

Another interesting analysis is the use of deictic words, like: "here, there, this, that, etc". During her debriefing, $\mathrm{N}$ said that she did not point and asked questions. However, in trial N3, she said: "Right here?", "Here?" and "this way.. this one" in three different occasions. Furthermore, in trial N2, she said: "This one?" and "This direction?". G, on the other hand, responded affirmatively to the same question and we found that in three occasions he asked: "That one?", and twice "Right here?". We do not know if in a similar task, a sighted person would make more use of this linguistic construction. We do not even know why $\mathrm{N}$ was not aware that she used such strategy. Maybe, this has something to do with the fact that speakers are often unwitting of gestures ([189] p 143). At this point, all we can say is that both participants used linguistic co-presence heuristics ([51] pp 32-35) just like any sighted person. The guide also used deictic expressions. In G1 alone, DO said "Right there" ten times. He used the same expression 9 and 4 times in trials G2 and G3 
respectively. As a matter of fact, this was the only deictic expression DO used. DA said "..in this direction.." four times in N1, one for each clue phrase word. In N2, she used the expression twice and again four in N3. DA also said: "..starts here.." eight times and “...here's the second letter..." seven times in all $\mathrm{N}$ trials combined.

Both $\mathrm{N}$ and G told us that because of the system, they asked fewer clarification questions. This measure is interesting because pointing is known to increase the precision of the utterances and therefore lower the likelihood of clarification questions (51] p 83). Table 3.8 shows the quantity of clarification questions asked per trial.

Table 3.8: Clarification questions

\begin{tabular}{|c|c|c|}
\hline Catch Phrase & Charade & Clarification questions \\
\hline Monkey see monkey do & G2 & 9 \\
& N3 & 13 \\
\hline My lips are sealed & G3 & 4 \\
& N2 & 9 \\
\hline Twinkle twinkle little star & G1 & 2 \\
& N1 & 7 \\
\hline
\end{tabular}

Interestingly, one can observe that $\mathrm{N}$ asked more clarification questions than $\mathrm{G}$. We have shown that $\mathrm{N}$ was more efficient when it comes to finding the clue phrase. Was asking clarification questions part of N's strategy? Did it help or hurt? To gain a better understanding on this matter, we had to analyze each clarification question asked. What we have noted is that $\mathrm{N}$ asked more questions because when she read a letter, instead of saying it, she would ask a clarification question. So, when she was reading the letter "B", instead of just saying "B", she would ask: "B?". G, on the other hand, would just say: "B". We regard the disparity as differences to personal style. We also could not see such difference helping or hurting one of the participant's performance. The reason for that is because the guide's response was similar in both cases. If the follower was right, the guide would proceed to the next letter or word. Otherwise, the guide would engage in expansion.

In this section, we went through the data available and tried to make comparisons with the answers the participants gave in the post-questionnaires. Most of the time, the data corroborated the information given by guides and followers. At other times, they were in apparent conflict. To resolve the conflict, we analyzed the data again in a more fine grained fashion. We could see that some pairs were more efficient than others. Then, we tried to learn what strategies worked and why. In the next section, we will try see if such strategies can be employed in classroom, particularly, in mathematics instruction. 


\subsection{Lessons learned}

DA/N's strategy was an interesting way of speeding things up. We are aware that our sensory replacement approach can only take us so far. Vision is a parallel sense while tactile exploration is necessarily a sequential activity. SBVI have to rely on sequential observations as "only part of the object can be seen or felt at a time", according to Osterhaus [206]. $\mathrm{N}$ knew the direction and size of the word before she moved to the second letter. On top of that, because of the spatial arrangement of the document (it is a grid of letters), she also knew what possible moves she could do to find the neighboring letters. It is early to affirm that the combination of the guide's clues, the raised line material and the system are enabling this "image build up" anticipation. What we can say is that the image build up is one of the core differences in the instruction of sighted and blind students. Obviously, the sighted has instantaneous access to images, while the blind has to go through this process.

What will happen when the students face illustrations with curves like the sine wave shown in figure 3.6? Is there a way to make a similar set of cues available to the students? This can be apparently hard. In the charade, the format of the document could be easily described via speech. The key issue might be having a previous knowledge about the shape of the figures used in instruction. So, maybe making the instructional material available before class will give the SBVI a chance to familiarize with the figures before class. Good instructors do that. Some even force or reward the students who read class notes, book chapters prior to classes. They are aware of the benefits early exposure brings to the students. Another aspect in graphs like the sine wave is that the raised lines form tracks that can be followed by the students' fingers. They can function as railroad tracks. Once you are on one, just keep going. The problem in railroad tracks is the intersections. They slow the train down and demands high chunks of the conductor's attentional resources. So we second Wall and Brewster's [279] call for the use of simple graphs, with no or few intersections.

We tried to make the case for a "clean figure" approach when preparing graphical instructional material. If this technology is ever to make its way to inclusive classrooms, it is important that educators refrain from overloading class notes. Maybe breaking down a complex graph into a series of simpler ones is the way to go. A figure that is too clogged can also bring perceptual difficulties to the sighted. In PowerPoint presentations, Stallings [253] advocates the use of no more than 7 topics per slides and each topic having 7 words at the most. Stalling's suggestions might be grounded on the work of Miller [194] on the limits of working memory - the author does not explicitly write that. Miller noticed that young adults can hold around seven elements, chunks, in their working memory. These chunks can either be digits, letters, words, or other units. Blokzijl and Andeweg [20] set out to find evidence for Stalling's and others' suggestions and found that content and format of the text slide does have a significant effect on the learning of the participants. However, they indicate, this effect can be overcome by the communication style of the presenter. Despite the work of Blokzijl and Andeweg, it is obvious that there is limit to the amount of textual information a slide must contain. The point is that this limit varies from one presenter to 
another as their presenting styles also vary. The studies listed above did not mention slides with figures.

Kalyuga et al [149] found that when different modalities, audio and vision in their study, present complementary and non-redundant information, they create the modality effect which increases effective working memory and positively impacts learning. Our initial studies showed that people can fuse the information acquired from both audio and tactile senses. We therefore hope that our approach will also create the modality effect.

\subsection{Transition}

We tried to make the case that our system enables similar grounding constraints to those of shared workspaces. To be more precise, because the parties are co-present, our approach enables more grounding constraints than those of shared workspaces and less than full copresence (SBVI). Nevertheless, we will again borrow another finding from shared workspaces to justify one concern our proposal raises: the delay cost. Kraut et al [163] manipulated the delay of updating the remote workspace of a user when it was the other user who performed the action. The authors observed that delaying the visual update in the space reduces benefits and degrades performance. A delay of only three seconds was reported to harm task performance. We observed delays below Kraut's threshold but most of them were above that. For Jefferson [138], an interval of one and a half seconds is "long" in spoken language. Furthermore, interruptions are perceived as annoying by speakers [53]. Long delays might have made the guides engage in expansion or try to fill the "silence gap" uttering words of encouragement. They seemed to be trying to speed things up. The problem might be the fact that the user was not able to decipher the glove directional signals in a timely fashion.

We hope that through extensive training, the haptic aided navigation task can turn from controlled to automatic making the extra cognitive load disappear. We also think that training can decrease the individual discrepancies. This is the theme of the next chapter. 
Chapter 4

\section{Enabling Embodied Skill through Game Playing}





\subsection{Introduction}

The charade results were not good enough. Participants spent a significant chunk of their conversational turns (around 30\%), discussing on how to reach the letter to which to the instructor was pointing. This is a clear evidence of discourse breakdown. Research shows that when conversants feel that the technology is "getting in the way" of their interaction, they are likely to quit using it [50, 53, 261]. We, therefore, understand that something must be done to increase the fluidity of the conversation in scenarios similar to those of the charade.

We hypothesize that this inordinate focus on the operational aspects of our assistive technology at the expense of the functional task of solving the cognitive problem is due to a lack of fluency and familiarity with the device. We had assumed that the blind students were able to employ the haptic device with minimal training, basing this on research on haptic devices that suggest a quick learning process (see Gallace [83]). With our device, for example, after a short period of familiarization subjects were able to correctly ascertain the direction indicated by the glove and to navigate to specific targets. This did not seem to be the case when the device was used to support situated discourse. Our pilot phrase charade study shows that this does not extend to blind students using our haptic device and engaging in the complex activity of multimodal discourse comprehension and production.

We posit that this is because deciphering of the directional signals is a secondary task performed in the service of discourse maintenance, and as such must become far more automatic. This is especially the case since the glove is used to support the understanding of multimodal discourse. Clark ([51], p. 140) suggests that the cognitive burden is heavier for the listener (follower) than for the speaker. In a sense, our device has to disappear in the sense of Dourish's concept of 'embodied skill'. Dourish writes: ([69] p 120), "embodied skills depends on a tight coupling between perception and action". The blind follower's attention to the signals from the glove must recede into the background, so that her conscious attentional and cognitive resources can be dedicated to the far more complex task of multimodal comprehension and discourse maintenance. Evidence suggests that when a haptic stimulus is presented in a dual-task scenario - where other concurrent information is being conveyed in a different mode, the result is a dramatical decrease in task performance [172, 251, 250]. The haptic aided navigation task should turn from controlled to automatic. The difference, according to Wickens ([291] pp 276-277), is that the controlled task demands attentional resources whereas the automatic does not. Wickens states that "extensive perceptual experience" and consistency of responses are necessary ingredients for a task to become automatic. Furthermore, extensive training helps to eliminate decrease in sensitivity [76] and improves task performance by improving tactile discrimination and increasing activation of the somatosensory cortical areas representing the stimulated body part [119].

Our preceding discussion suggests that we need an approach that promotes extensive use of the glove for a haptic navigation task to promote more immediate perception-action coupling. 
Our participants who are blind have expressed strong motivation to use our glove technology to improve their learning experience. This long term goal, however, requires a process of skill acquisition that may be tedious and mundane. Csikszentmihalyi, in his discussion of flow and enjoyment, asserts that such long-term goals can be served by creating a series of shorter-term activities with clear goals and feedback to maintain motivation (60] pp. 54-58). We explore the use of self-regulated gameplay to provide this 'motivation scaffold' so that the participant is able to achieve the level of skill necessary by engaging in a pleasurable activity that she understands will contribute to her ultimate motivation to support the development of technology to aid learning experiences for individuals who are blind.

In this chapter, we discuss how games were employed to help people with disabilities to develop skills and where our approach differs from previous research. After that, we show how Csikszentmihalyi's flow theory influenced our game design. We then proceed to discuss the studies we conducted using the game. We then conclude that the gamers were able to navigate faster, more accurately and had fun. Finally, we ascertain that such gains were transferrable to scenarios like the charade.

\subsection{Games and Accessibility}

Buaud [34] sees games is a important aspect of youth culture. For Csikszentmihalyi [59], "Play allows a young organism to experiment a repertoire of behaviors in a non-threatening environment and, hence, to learn by trial-and-error without paying too high a price for errors". In fact, people from all ages enjoy playing games because they elicit good feelings like being in the Flow, which is a state of total absorption in an activity and concentration that can result in a "deep enjoyment" [6, 142] or Presence, which is a "subjective experience of being in place when physically situated in another" [298]. What is appealing for those with disability is that in this new place, where there is no disability. For Archambault [4], accessible games can assume different roles: Ludic, educational and therapeutical. In this section, we discuss previous research and how they can inform us.

Enabling those with disabilities to play games seems interesting from the industry point of view. First, because of the size of the market. According to an 2004 United Nations report [203], $10 \%$ of the world population have some kind of disability. Academia and industry seem to be responding to that. According to Archambault [6], in the last 10 years over 400 audio-games have been developed. The International Game Developers Association ( $I G D A)$ created the Game Accessibility $S$ pecial Interest group ( $G A-S I G$ ), with the purpose of "methods of making all game genres universally accessible to all, regardless of disability". A GA-SIG survey [19] found 16 games especially designed for those who are blind and other 16 for those with low vision.

Some research projects have tried to make accessible versions of famous games. Access Invaders [101] is an accessible implementation of the famous game Space Invaders which can be 
played by gamers with different disabilities (eg. Visual impairment, mild memory/cognitive impairment as well as hand-motor deficiencies). Blind hero [301] is an adaptation of the also famous Guitar Hero which is played with a haptic glove (similar to ours). AudioQuake [10], a first person shooter game, is also an adaptation (from Quake). People with visual disability can play both AudioQuake and Terraformers [284] against an opponent with no disability. The AudioQuake authors' goal is to "make mainstream games accessible by adapting underlying information and rendering it in an appropriate format". For them, this approach enables on-line collaboration between blind and sighted and can be useful in education. There are other games developed specially for the blind, like the drive project [271], designed for blind children of the age of 10 to 14 whose goal is "to create a (non-visual) computer game that resembles to same level of accessibility and fun as a game with visual output".

Archambault [6] sees accessible on-line games as a great opportunity to promote social inclusion because gamers with disabilities can play on-line with non-disabled peers. This bears some resemblance to our work as the choice of adequate interface can promote inclusion. In this realm, we have the "games designed for all" [6], such as UA-Chess [102], which can be played by gamers with different disabilities. An important hurdle, according to GA-SIG, is that mainstream games are not compatible with more specialized devices like specialized joysticks, eye-trackers, gloves, or even brain signals. The group proposed guidelines to create accessible games. Such guidelines were later refined by Ossmann and Miesenberger [205].

Other accessible games were designed for educational purposes instead of pure fun and social engagement. Audiomath [233] is one example. Its goal is to enhance spatial memory, and consequently mathematics, by enabling the use of space to organize concepts. Grammenos and collegues [101] advocate the use of inclusive design techniques. McElligott and Van Leeuwen [184] had children who are blind and with learning disabilities playing active roles in the design of games and toys. The TIM project 34] produced a tactile board on which blind kids can play several games: Reader rabbit's toddler [5] and Findit [216]. FindIt gave rise to a Game Generator [216] which enables educators to create their own games.

In therapeutic, games have been used in conjunction with virtual reality to help people with special needs developing new skills [169], like going to the mall [125], simulating street crossing [124] and learning how to browse the internet [229]. The strategy was also used in rehabilitation like learning how to use a wheelchair [125] or a cane [169].

Games have also been used to investigate how users react/adapt to a novel form of interaction. Sepchat and colleagues [243] created a maze game to help users get used to braille displays. Wang and Hayward [281] devised a memory game to help them assessing the capabilities of their tactile transducers. Johansson and Linde [141] also developed a labyrinth game to investigate if Microsoft's Sidewinder Force Feedback is useful to visualize objects. Raisamo et al [220] used the same approach to test Logitech's WingMan Rumblepad. Sjostrom [247] created the Memory House game to "get an idea of how useful it can be to include haptics in a computer interface for blind people". Iglesias and colleagues [123] created an adventure game to "demonstrate the validity of the approach (audio-haptic 
applications)". In Iglesias' game, players have to find bombs and deactivate them by touch. Researchers have applied the gaming strategy with people with disabilities in several different scenarios. The use of games in rehabilitation seems similar to ours as participants play games to develop new skills. We shall now introduce our skill developing game.

\subsection{Designing for flow Experience}

As explained in the previous section, the skill training game is meant to be played by the student who is blind as a skill training tool. The game is designed to produce a set of graduated challenges that engages the player at successive levels of difficulty as her skill improves. Gamers should become attracted to the game to enable the extensive training. To aid in this design, we employed the "Flow" concept of "Optimal Experience" developed by Csikszentmihalyi [60].

The game is designed to produce a set of graduated challenges that engages the subject at successive levels of difficulty as the level of skill improves. To aid in this design, we employed the "Flow" concept of "Optimal Experience" developed by Csikszentmihalyi [60]. Csikszentmihalyi's model has been adapted to the design of computer games [41]. Flow is a feeling of "complete and energized focus in an activity, with a high level of enjoyment and fulfillment" [41]. Flow lies in the balance of anxiety and boredom produced by task challenge and player abilities [41, 139]. It is an individual experience [59]. It is a state of total absorption in an activity which elicits deep enjoyment [142]. When we are in the "flow zone", we are so engaged and focused that we lose "track of time and worries" [41]. In other words, we are in the "zone" when we performing an activity that gives us pleasure and, at the same time, demands our attention. Csikszentmihalyi lists the components of the Flow:

- A challenging activity requiring skill;

- A merging of action and awareness;

- Clear goals;

- Direct, immediate feedback;

- Concentration on the task at hand;

- A sense of control;

- A loss of self-consciousness; and

- An altered sense of time. 
Csikszentmihalyi's model does not require that an activity exhibit all eight components to enable the flow experience. The goal of our skill training game design was to provide a set of challenging task, graduated to improve the participant's skill in the use of our haptic glove. We employ Csikszentmihalyi's model of 'challenge' where he states: "Enjoyment appears at the boundary between boredom and anxiety, when the challenges are just balanced with the person's capacity to act." ([60], p. 52). Hence, our design objectives were to provide a set of clear goals with direct and immediate feedback. The task had to be tightly associated with our overall purpose of encouraging the development of embodied skill in the use of the glove. In doing so, we seek to merge the actions of the player with the awareness of the pragmatic goal (shared by our subjects) that our system will eventually aid them in learning, by providing clear near-term goals that they can achieve, provide timely feedback of their level of skill improvement, require some degree of commitment and concentration to play, and provide the subject with a sense that they are in control of the game.

\subsubsection{Designing Challenging Activity}

We modeled our game in a fantasy context patterned loosely on the television and movie series: "Mission Impossible". Table 4.1 shows the game setup that is read to the player who is blind using Text-to-Speech when the player starts the game. We make extensive use of speech and sound effects as a means to enable engagement by providing a multimodal experience. The subject has to move her hand over a 'gameboard' under the guidance of the haptic glove system. When her hand comes within some level-defined distance from the target, the 'bomb' is disarmed. Target's coordinates are randomly assigned.

Table 4.1: Game instructions for the first phase

This is Agent Smith of the Agency of Impossible Missions (AIM). Your services are needed at this time. Dr. Evil has stolen all of the launch codes of our nuclear arsenal. With such codes he can destroy any city in the world. Your mission, if you decide to accept it, is to disarm the launch sequences as they are detected. The first launch sequence detected is for New York City. You have 2 minutes to find the codes and disarm them. Go!

The challenge and the goals are clear. In this situation, the goals are not only to find the targets, or to play the game per se. It is about helping in the construction of a technology that might be useful in the teaching of the blind in general. This kind of feeling lasts longer and is more rewarding than the immediate and temporary satisfaction of a regular game play, and provides the student with a broader awareness of how the game will improve the technology available to students who are blind (Csikszentmihalyi's second Flow component).

The direct and immediate feedback is provided by the tracking and the haptic glove. The need for concentration is clear: There is a goal to be accomplished within a time frame, the 
player has to focus on the task or she will fail. The player can quit the game at any time by pressing any key on the keyboard. She can also pause the game by moving her hand out of the tracking area. This is meant to give the player a sense of control.

The game comprises three levels. Each level lasts 2 minutes. The player must find the codes and disarm a number of warheads at each level within that time. In level one, ten targets must be found within the 2-minute timeframe. In levels two and three the number of targets to be found within the same time frame are 20 and 40 respectively, and the required distance of the hand from the target point decreases successively. Figure 4.1 shows target sizes and distances from the hand on levels one and two.

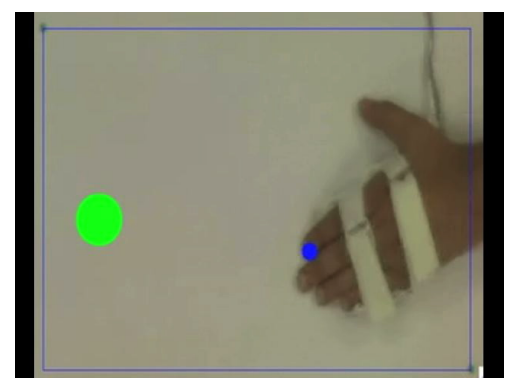

(a) Level 1. Bigger and closer targets. Only vertical and horizontal movements.

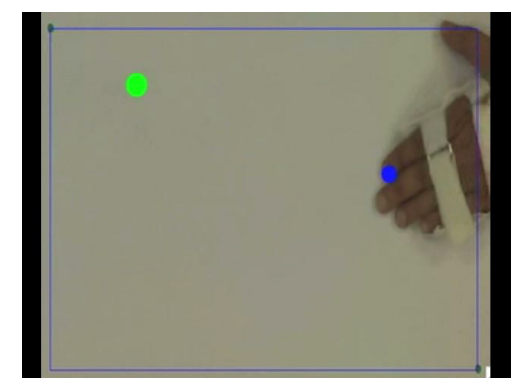

(b) Level 2. Targets got smaller and further away. Diagonal movements are necessary.

Figure 4.1: The game for blind

When the player gets to the target the system emits a sound of a 'small detonation' indicating the destruction of the target device. Together with the Mission Impossible theme music, this audio feedback is designed to enhance the sense of immersion in the game.

The program also speaks the player's score when a target is reached. The score is calculated by the simple formula 4.1 .

$$
\text { points }=\text { distance to target/time to target (in milliseconds) }
$$

The faster the player navigates, the more points she earns. The program records the highest score each player accumulates, along with the most advanced level attained. To add a degree of competition, the system speaks the names and scores of the top three players before articulating the game instructions. This is to increase the competition and also to function as a stimulus. If the player happens to be ranked number 1, the program compliments her and says: "don't rest on you laurels, there is room for improvement".

Since the game is inspired on the Mission Impossible movie series, after the "Go" command the movie's theme song starts playing. The music tempo is paced according to the game level. 
For instance, when the player reaches level two, the background music tempo is increased by $50 \%$. On level three, the tempo is $75 \%$ faster than that at level one. The idea is based on the work of Brodsky's [29]. He studied the effects of altering the tempo of a background music on simulated driving and found that as the tempo of the music increases, so does the simulated driving speed. In more advanced levels the player is required to navigate faster, so this change in tempo functions as an extra stimulus.

\subsubsection{The Flexible Game Structure}

Being in the flow is a personal and individual experience. What can be challenging to one might be boring to another. Moreover, what can be challenging to a person at one time can become boring to that same person minutes later. Hence, the game was built around a central data structure that allows easy tuning of its core parameters. The structure is a list of levels in the form of the tuple shown in table 4.2. We initially set the value of $n$, number of levels, to three. To add entry on this list means to add a new level to game.

Table 4.2: Basic game tuple

Level $(n)=$ (Number of targets, Target size, Minimum distance to target, Total time alloted, Minimum distance to borders, Instructions, Playback music, Victory music)

With such structure it is facile to make the game easier or harder to play. One possible way to make it harder is to add more targets to be decoded in a shorter amount of time. Another is to make the targets smaller, or to add more levels. We will now define and discuss each parameter.

Number of targets is the total amount of targets at that particular level. The program presents the targets one at a time. When the player gets to that target, another one is presented at a random position. The player advances to the next level when she finds all the targets within the total time alloted (another parameter).

Target size is measured in pixels. The target is the circle in figures $4.1 \mathrm{a}$ and $4.1 \mathrm{~b}$, Target size is the circle radius. The target is considered found when the blue circle's (same figures) center enters the circle's area.

Minimum distance to target is the minimum distance (in pixels) from a target to the player's tracked finger. The program randomly generates the targets' coordinates. However, 
only the targets whose distances to the player's tracked finger are greater than this parameter are presented to the user.

Total time alloted is measured in milliseconds. It is the total time the player has to find all targets in a given level.

Distance from borders is the minimum distance (in pixels) a target has to be from the borders of the tracking area. The closer the target is to the borders the harder it is to find it.

Instructions are spoken to the player before she starts a particular level. This parameter is useful if we want to change the game's story line.

Background music to be played in the background while the player is in a given level. Each level can have its own background music.

Victory music is played every time a player finishes a level. When this occurs, the program congratulates the player and invites her to take a moment and dance the victory dance.

\subsubsection{The game levels}

The setting of the game parameters is a matter of trial and self experimentation. Since we want our players to enter Csikszentmihalyi's zone, we tried to enter the zone ourselves while testing different settings. We followed Chen's [41] advice and set the parameters so that the activity does not get too challenging, which would raise the level of anxiety nor too easy, which will lead to boredom (figure 4.2).

Table 4.3 shows how we initially set the parameters. As the player progresses from one level to the next, there is a significant increase in challenge. In level two, the players have to find twice the number of targets which are twice as distant from her tracked finger and half closer to the borders, in the same amount of time. 


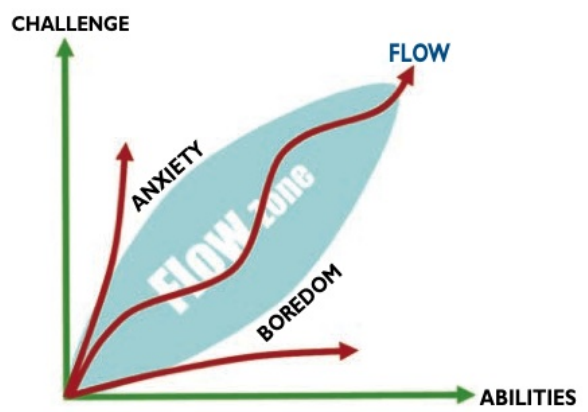

Figure 4.2: Flow Zone Factors, from Chen[41]

Table 4.3: Game initial parameters

\begin{tabular}{|c|c|c|c|c|c|c|c|c|}
\hline Phase & $\begin{array}{l}\quad \# \\
\text { of } \\
\text { Tgts }\end{array}$ & $\begin{array}{l}\text { Tgt } \\
\text { size }\end{array}$ & $\begin{array}{l}\text { Min } \\
\text { Dst } \\
\text { to } \\
\text { Tgt }\end{array}$ & Time & $\begin{array}{l}\text { Dist } \\
\text { to } \\
\text { Bor- } \\
\text { ders }\end{array}$ & Instructions & $\begin{array}{l}\text { Playback Mu- } \\
\text { sic }\end{array}$ & Victory Music \\
\hline 1 & 10 & 1000 & 120 & $2 \mathrm{~min}$ & 200 & See table 4.1 & $\begin{array}{ll}\text { Mission } & \text { Impos- } \\
\text { sible } & \end{array}$ & $\begin{array}{ll}\text { a cowboy } & \text { yelling } \\
\text { "yeehaw" }\end{array}$ \\
\hline 2 & 20 & 200 & 240 & $2 \mathrm{~min}$ & 100 & Same instructions. Different city & $\begin{array}{l}\text { Mission Impos- } \\
\text { sible with tempo } \\
\text { increased in } 50 \%\end{array}$ & $\begin{array}{l}\text { a cowboy yelling } \\
\text { "yeehaw" }\end{array}$ \\
\hline 3 & 40 & 10 & 480 & $2 \min$ & 50 & Same instructions. Different city & $\begin{array}{l}\text { Mission Impos- } \\
\text { sible with tempo } \\
\text { increased in } 75 \%\end{array}$ & $\begin{array}{l}\text { a cowboy yelling } \\
\text { "yeehaw" }\end{array}$ \\
\hline
\end{tabular}

\subsection{The trials}

We wanted the game to become an attraction for the SBVI community on campus and mitigate our shortage of participants. Therefore, the game was used as both a recruiting and training tool. Trained participants will be needed in future studies related to this project. After this game study, we wanted to repeat the charade study with the participants who played the game and to compare their performance against the data reported earlier. Furthermore, we wanted them to participate in a capstone mathematics instruction experiment. To satisfy these pragmatic concerns, it was critical that we provided flexibility of schedule to our participants. To achieve this, we developed a strategy of deploying a game arcade to which the subjects can go at their convenience. The program and all the required equipment were stationed in a room reserved especially for this purpose. All the student needed to do was schedule a time that worked for both her and her tutor (who also collected the experiment data). Each participant was required to play the game for three one hour long sessions in three different days. However, if any participant really felt challenged by the game and wanted to come back a fourth or fifth time, she was allowed to. 
In total, we had five participants, three females (A, N and $O$ ) and two males ( $R$ and $G$ ). N, $\mathrm{R}$ and $\mathrm{G}$ also participated on the first charade experiment.

\subsection{The Gamers' Experience}

The participants were requested to answer a post-questionnaire after their third trial. At that point, the study was "officially" over, although the participants could schedule more sessions, if they wished. $\mathrm{R}$ was the only one who returned after answering the questions. He had four more sessions (because he enjoyed the game, and wanted to earn the highest score) and data from these were also collected by the computer program. For consistency, we include only his questionnaire completed at the end of his third session in our results.

We proposed fifteen statements to the participants and asked them to rate their level of agreement with them. The rating ranged from 1 (totally disagree) to 5 (totally agree), 3 being neutral or no opinion. Participants were also urged to comment on the statement being presented. The questionnaire can be found on page 225.

All strongly agreed that they would perform better if they had played more. All but one either agreed or strongly agreed that they had to stay very alert to get the targets on time. $\mathrm{R}$ told us that if this game were in the market, he would buy it. All strongly agreed that they had fun playing the game and that they would like to return and finish it. "Finishing" means getting through all the three levels of the game. Our engagement argument also found evidence in the fact that all participants considered that they got so absorbed by the game that they lost track of time and strongly agreed the they had full control of their character to accomplish the mission. However, none of them forgot that they were wearing a glove.

Three participants did not find level 1 challenging. This is what we expected. Level 1 was designed as the "warm-up" level to bring all subjects to a baseline of proficiency. For some of the participants, this was the very first time they had contact with the haptic glove. Half of the participants found level 2 challenging whereas all of those who reached the third level, strongly agreed that it was challenging. As for boredom, level 1 was considered not boring by 4 of the 5 participants and that was the unanimous opinion for levels 2 and 3. We also asked for how long they could keep playing. The possible answers ranged from 1 (5 minutes) to 4 (30 minutes) and 5 (45 minutes or more): All but one agreed that they could play 45 more minutes or more beyond the time they just played, without feeling bored. The only person who gave a different answer $(30 \mathrm{~min})$, $\mathrm{O}$, had just played for more than 54 minutes without interruption. She was trying to break the overall record. 


\subsection{Data Analysis}

The quantitative data, automatically collected every time the game was played, consisted of 1) the elapsed time since the game began; 2) $\mathrm{x}$ and $\mathrm{y}$ coordinates of the player's tracked finger, 3) the $\mathrm{x}$ and $\mathrm{y}$ coordinate of the target. This allowed us to analyze the navigation speed, the techniques participants used to locate the targets, how they changed the way they navigated to the targets as they became more skillful. The data discussed here are from game play at level two. We feel they hold the most value for an overall comparison because all participants reached this level and found it challenging.

We begin our discussion examining the techniques used by the participants to locate a target. For each target a map was produced to show the path participant took from the starting point to the target. These data were then categorized into groups. The techniques that emerged from the data were: Direct (navigation straight to the target - figure 4.3a); overshoot (the player passes by the target and returns - figure 4.3b); stair step (subject follows the directional signals strictly - figure 4.3c); Unknown (technique does not fit into any of the previous categories - figure 4.3d) and Lost (figure 4.3e).

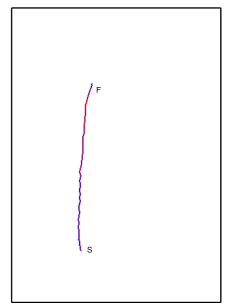

(a) Direct

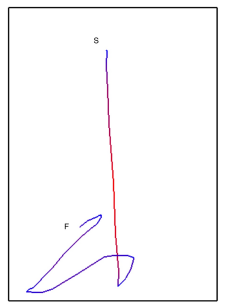

(b) Overshoot

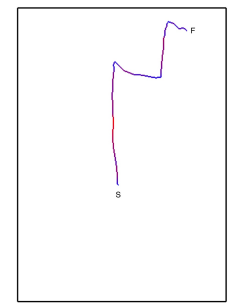

(c) Stair Step

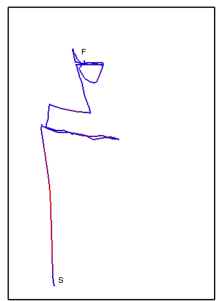

(d) Unknown

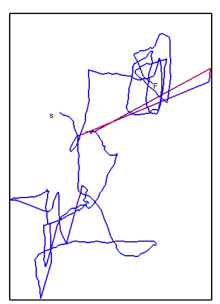

(e) Lost

Figure 4.3: Different techniques for finding targets

As expected, the Direct technique yielded significantly shorter times to target than all the others (paired Student's $t, \mathrm{df}=4, \mathrm{t}=2.501, \mathrm{p}$-value $<0.0001$, see table 4.4 ) $)^{1}$ and also the shortest distance travelled (paired Student's $t, \mathrm{df}=4, \mathrm{t}=2.054$, $\mathrm{p}$-value $<0.0001$, see table 4.5).

\footnotetext{
${ }^{1}$ All statistical tests reported on this chapter were performed at a $95 \%$ confidence interval
} 
Table 4.4: Navigation Techniques and Time to Target

\begin{tabular}{|lrrrrr|}
\hline Technique & $n$ & $\begin{array}{c}\text { Mean } \\
\text { Time }(\mathrm{ms})\end{array}$ & Std Error & $\begin{array}{r}\text { Lower } \\
95 \%\end{array}$ & $\begin{array}{r}\text { Upper } \\
95 \%\end{array}$ \\
\hline Direct & 364 & 3260.80 & 398.00 & 2479 & 4042.00 \\
Overshoot & 249 & 5493.10 & 481.00 & 4549 & 6438.00 \\
Stair Step & 534 & 5690.70 & 328.70 & 5045 & 6336.00 \\
Unknown & 57 & 6452.30 & 1002.70 & 4483 & 8422.00 \\
Lost & 130 & 13074.60 & 665.60 & 11767 & 14382.00 \\
\hline
\end{tabular}

Table 4.5: Techniques used in second level and Distance Travelled

\begin{tabular}{|lrrrrr|}
\hline Technique & $\mathrm{n}$ & $\begin{array}{r}\text { Mean } \\
\text { Distance }\end{array}$ & $\begin{array}{c}\text { Std } \\
\text { Error }\end{array}$ & $\begin{array}{c}\text { Lower } \\
95 \%\end{array}$ & \multicolumn{1}{c}{$\begin{array}{c}\text { Upper } \\
95 \%\end{array}$} \\
\hline Direct & 364 & 3260.80 & 398.00 & 2479.00 & 4042.00 \\
Overshoot & 249 & 5493.10 & 481.00 & 4549.00 & 6438.00 \\
Stair Step & 534 & 5690.70 & 328.70 & 5045.00 & 6336.00 \\
Unknown & 57 & 6452.30 & 1002.70 & 4483.00 & 8422.00 \\
Lost & 130 & 13074.60 & 665.60 & 11767.00 & 14382.00 \\
\hline
\end{tabular}

We wondered if participants had migrated from a less to a more effective technique as the trials progressed. Table 4.6 shows techniques and their usage from trials one through three, for game level 2. One can observe increases in Direct and Stair Step techniques along with a decrease in Overshoot. The number of times participants got lost remained roughly the same. The improvement could also be noticed in significant shorter times to target. The mean times to target were: 6205.94, 5029.79 and 4685.09 millisecond for trials 1, 2 and 3 respectively. The difference from day 1 to day 2 was statistically significant (paired Student's $t, \mathrm{df}=2, \mathrm{t}=1.801$, $\mathrm{p}$-value $<0.0001$, tables 4.6 and 4.7 ).

Table 4.6: Technique per Trial and Time to Target

\begin{tabular}{|ccccccc|}
\hline Trial & \multicolumn{4}{c}{ Technique in \% } & \multicolumn{2}{c|}{ Time $(\mathrm{ms})$} \\
& Direct & Over- & Stair & Unk. & Lost & Mean \\
& & shoot & Step & & & \\
\hline $\mathbf{1}$ & 18.82 & 41.18 & 31.76 & 4.71 & 3.53 & 6205.94 \\
$\mathbf{2}$ & 25.45 & 23.18 & 43.18 & 5.00 & 3.18 & 5029.79 \\
$\mathbf{3}$ & 24.47 & 26.60 & 42.55 & 3.19 & 3.19 & 4685.09 \\
\hline
\end{tabular}

Table 4.8 shows participants and the navigation techniques while playing at level 2. Despite the fact that N was the one who most used the Direct technique (46.32\% of the time), she was the slowest gamer (paired Student's $t, \mathrm{df}=4, \mathrm{t}=3.122, \mathrm{p}<0.0001$ ). Interestingly, $\mathrm{N}$ was the game's top scorer during all her 3 trials. This was due to our flexible scheduling. Only 
Table 4.7: Trials and Time to Target

\begin{tabular}{|cccrcc|}
\hline Trial & $n$ & $\begin{array}{c}\text { Time }(\mathrm{ms}) \\
\text { Mean }\end{array}$ & $\begin{array}{c}\text { Std Error } \\
\text { Error }\end{array}$ & $\begin{array}{c}\text { Lower } \\
95 \%\end{array}$ & $\begin{array}{c}\text { Upper } \\
95 \%\end{array}$ \\
\hline $\mathbf{1}$ & 271 & 6205.94 & 211.51 & 5791.20 & 6620.70 \\
$\mathbf{2}$ & 420 & 5029.79 & 169.88 & 4696.70 & 5362.90 \\
$\mathbf{3}$ & 643 & 4685.09 & 137.27 & 4415.90 & 4954.30 \\
\hline
\end{tabular}

after she was done, competition heated up and improved the gains. Other significant speed differences were found, refer to table 4.9 for intervals and ranking.

Table 4.8: Participants and Navigation Techniques

\begin{tabular}{|crrrrr|}
\hline Participant & Direct (A) & $\begin{array}{c}\text { Direct (B) } \\
\text { OverShoot }\end{array}$ & Stair Step (C) & A+B+C & Other \\
\hline A & $20.59 \%$ & $2.94 \%$ & $50.00 \%$ & $73.53 \%$ & $26.47 \%$ \\
G & $20.31 \%$ & $31.25 \%$ & $28.13 \%$ & $79.69 \%$ & $20.31 \%$ \\
N & $\mathbf{4 6 . 3 2} \%$ & $12.63 \%$ & $33.68 \%$ & $92.63 \%$ & $7.37 \%$ \\
O & $29.09 \%$ & $38.71 \%$ & $16.13 \%$ & $83.93 \%$ & $16.07 \%$ \\
R & $15.51 \%$ & $64.17 \%$ & $12.83 \%$ & $92.51 \%$ & $7.49 \%$ \\
\hline
\end{tabular}

Table 4.9: Navigation Speeds for game level 2

\begin{tabular}{|ccccrrr|}
\hline Rank & Partc & $n$ & Speed & Std Error & Lower & Upper \\
& & & & & $95 \%$ & $95 \%$ \\
\hline 1 & A & 165 & 4.21 & 0.15 & 3.92 & 4.51 \\
2 & O & 114 & 3.93 & 0.18 & 3.57 & 4.29 \\
3 & R & 485 & 3.73 & 0.09 & 3.56 & 3.90 \\
4 & G & 459 & 3.59 & 0.09 & 3.42 & 3.77 \\
5 & N & 111 & 2.75 & 0.18 & 2.39 & 3.11 \\
\hline
\end{tabular}

The fastest three (O, A, and R) were able to drastically increase their navigation speed (45\%, $45 \%$, and $62 \%$ increase, respectively). They were also those who achieved level 3 and were the most competitive. $\mathrm{R}$ asked us to e-mail him in case someone had broken his record. A's last trial lasted almost one and half hours as she wanted to make sure that she was the top scorer.

Another interesting comparison is between participants and overall distance travelled. Table 4.10 shows data from game play at level 2. One can see that $\mathrm{A}$ and $\mathrm{O}$ travelled shorter distances (some differences are significant). This is so because $\mathrm{A}$ and $\mathrm{O}$ always moved their 
Table 4.10: Participants and distance travelled at game level 2

\begin{tabular}{|crrlrr|}
\hline Participant & $\mathrm{n}$ & $\begin{array}{l}\text { Mean } \\
\text { Distance }\end{array}$ & $\begin{array}{l}\text { Std } \\
\text { Error }\end{array}$ & $\begin{array}{r}\text { Lower } \\
95 \%\end{array}$ & $\begin{array}{r}\text { Upper } \\
95 \%\end{array}$ \\
\hline $\mathrm{A}$ & 94 & 375.26 & 47.62 & 281.70 & 468.82 \\
$\mathrm{G}$ & 380 & 651.65 & 23.64 & 605.21 & 698.09 \\
$\mathrm{~N}$ & 261 & 438.78 & 28.49 & 382.81 & 494.75 \\
$\mathrm{O}$ & 85 & 292.71 & 49.87 & 194.73 & 390.70 \\
$\mathrm{R}$ & 514 & 488.57 & 20.30 & 448.68 & 528.46 \\
\hline
\end{tabular}

hand to the center of the tracking area every time they found a target. This shortened the distance to any target.

The current trend in the field of vibro-tactile displays is to keep the signal patterns as simple as possible [83, 12, 31]. Our glove can either convey eight different directional signals (North, Northeast, East, etc), or only four (N,E,S,W). We, therefore, wanted to know which signaling pattern would yield better performance. Among the three trials, the participants were required to test both signaling patterns. They could do 2 trials with one pattern and 1 with the other. To compare the efficiency of the patterns, we looked at the ratio between the straight-line distance to the target and the length of the path taken to the target. Therefore, whenever the participant took a direct, straight path to the target this ratio would equal one. We found no significant different related between the two signaling patterns. Table 4.11 shows the intervals.

Table 4.11: Signaling Patterns and Distance Travelled

\begin{tabular}{|clrrrr|}
\hline $\begin{array}{c}\text { Number of } \\
\text { Directions }\end{array}$ & & Ratio & $\begin{array}{l}\text { Std } \\
\text { Error }\end{array}$ & $\begin{array}{r}\text { Lower } \\
95 \%\end{array}$ & $\begin{array}{r}\text { Upper } \\
95 \%\end{array}$ \\
\hline 4 & 864 & 0.60 & 0.01 & 0.59 & 0.62 \\
8 & 470 & 0.62 & 0.01 & 0.60 & 0.64 \\
\hline
\end{tabular}

When the glove is signaling 8 directions, participants reached targets in less time (One way Anova, F Ratio $=5.4304, \mathrm{f}=0.0199$; table 4.12 .

Table 4.12: Number of directions and time to target

\begin{tabular}{|rrrrrr|}
$\begin{array}{r}\text { Number of } \\
\text { Directions }\end{array}$ & $\mathrm{n}$ & $\begin{array}{l}\text { Time }(\mathrm{ms}) \\
\text { mean }\end{array}$ & $\begin{array}{l}\text { Std Error } \\
\text { Error }\end{array}$ & $\begin{array}{l}\text { Lower } \\
95.00 \%\end{array}$ & $\begin{array}{l}\text { Upper } \\
95.00 \%\end{array}$ \\
\hline 4 & 864 & 5920.87 & 176.08 & 5575.5 & 6266.2 \\
8 & 470 & 5333.92 & 180.1 & 4980.7 & 5687.2 \\
\hline
\end{tabular}

We also wondered if different signaling patterns would mean different navigation techniques. 
As one could expect, when receiving only 4 directions, gamers tend to use less the direct and more the stairstep technique. However, those receiving 8 directional signals overshot targets in $50.57 \%$ of the time, against $22.81 \%$ for four directions (table 4.13 ).

Table 4.13: Number of directions and Navigation Techniques

\begin{tabular}{|lrr|}
\hline Technique & \multicolumn{2}{c|}{ Num directions } \\
& 4 & \multicolumn{1}{c|}{8} \\
\hline Direct & $19.65 \%$ & $26.44 \%$ \\
Overshoot & $22.81 \%$ & $50.57 \%$ \\
Stairstep & $48.77 \%$ & $18.39 \%$ \\
Unknown & $5.26 \%$ & $2.30 \%$ \\
Lost & $3.51 \%$ & $2.30 \%$ \\
\hline
\end{tabular}

In summary, we observed important performance gains in all participants. For some, the gains were more pronounced than for others. We posit that competition played an important role in maximizing the gains. Gamers were able to navigate more efficiently as the trials progressed. Finally we evaluated the efficacy of two different directional signaling patterns (4 and 8 directions). We found that gamers were faster when receiving the 8 direction pattern but not more precise. Probably if there were more trials, we would have seen improvement in accuracy too as all participants agreed that there was still room for improvement. Interestingly, though, is the fact that all participants who reached game level 3 preferred to use the glove set for 4 directions. They said that they needed to be extremely precise because the target size was very small and they did not want to overshoot. Some participants had their own signaling preferences, some preferred 4 while others 8 (during game level 2). When asked to use the non-preferred pattern they were reluctant because of the competition. A change in the signaling would slow them down.

\subsection{Revisiting the Phrase Charade}

Our game results show performance gains in playing the game. However, we needed to know whether the gains were transferable to the lecture-like task scenario. To answer it, we asked our participants to redo the charade experiment. We conducted this second charade study in the late Spring of 2009. This time, we used catch phrases ranked from 5-8 from table 4.14the top four catch-phrase were used in the first charade study. The experimental procedure remained absolutely the same.

Among those who participated in the second charade study, three of participants ( R, N, and $G$ ) were also present on the first charade. We had one dropout since the first charade: M. M was not invited for the following studies (game and 2nd charade) because she has 
Table 4.14: Catch and Clue phrase pairs

\begin{tabular}{|ccc|}
\hline Rank & Catch Phrase & Clue Phrase \\
\hline 1 & Twinkle twinkle little star & Blink blink small sun \\
2 & Monkey see monkey do & Gorilla look gorilla execute \\
3 & My lips are sealed & Me mouth be shut \\
4 & Never a dull moment & Nevermore one dumb instant \\
5 & Out of this world & In the next galaxy \\
6 & See you later alligator & In a while crocodile \\
7 & Don't hold your breath & No stop thy respiration \\
8 & One for the road & Uno to a route \\
\hline
\end{tabular}

other disabilities which cause loss of sensation on her palm. On the other hand, we had two new participants joining our studies starting from the game $(\mathrm{A}, \mathrm{O})$. Therefore, we shall first compare the pre and post game performances of R, N and G. Then, we report our analysis of the overall performance times, general discourse content, and participant experience.

\subsubsection{Performance Gains and Task Focus}

We compared the overall completion times of the phrase charade performed by our three subjects from the Spring of 2007 against that of our second phrase charade study. Each participant solved three charades in each of our studies, giving us 9 sets of results in our 2007 study, and potentially 15 sets of results from our 2009 study. One dataset from a repeat subject in the second charade had to be excluded because of a system malfunction during that study. This yields 8 sets of data from the repeat participants and 6 from the new participants in the 2009 study.

The performance times were markedly increased in the second charade. For our repeat subjects, the new mean completion time was 238.50 seconds as opposed to 443.88 seconds from the 2007 study. This is a 1.86-fold time difference. A paired Student's $t$ test at a $95 \%$ confidence interval show the obvious significance of this result (paired Student's $t$, $\mathrm{df}=8, \mathrm{t}=2.1650, \mathrm{p}$-value $=0.0020)$. When we include all 5 subjects in the 2009 study, we have an mean completion time of 251.92 seconds. Table C.1 on page 219 shows a complete listing of the catch/clue phrases used by each participant in both charade studies along with the times to complete them.

Our observations of the discourse content in the 2009 study showed virtually no references to the technology in the 2009 study. Almost all the speech was dedicated to solving the charade. In other words, the mean of conversational turns devoted to problem was $96.68 \%$ among the repeat subjects. In the first charade trial, the mean was $66.7 \%$, and significantly different (paired Student's $t$ test, $\mathrm{df}=8, \mathrm{t}=2.058, \mathrm{p}$-value $<0.0001$ ). When we include all participants, we have an mean of $97.14 \%$ of the turns dedicated to problem solving. Figure 4.4 shows the percentages of problem solving (object level) turns of the two charade studies 
(before and after playing the game). Table C.2 on page 220 shows the percentages of turns devoted to solving the charades for each participant in both studies.

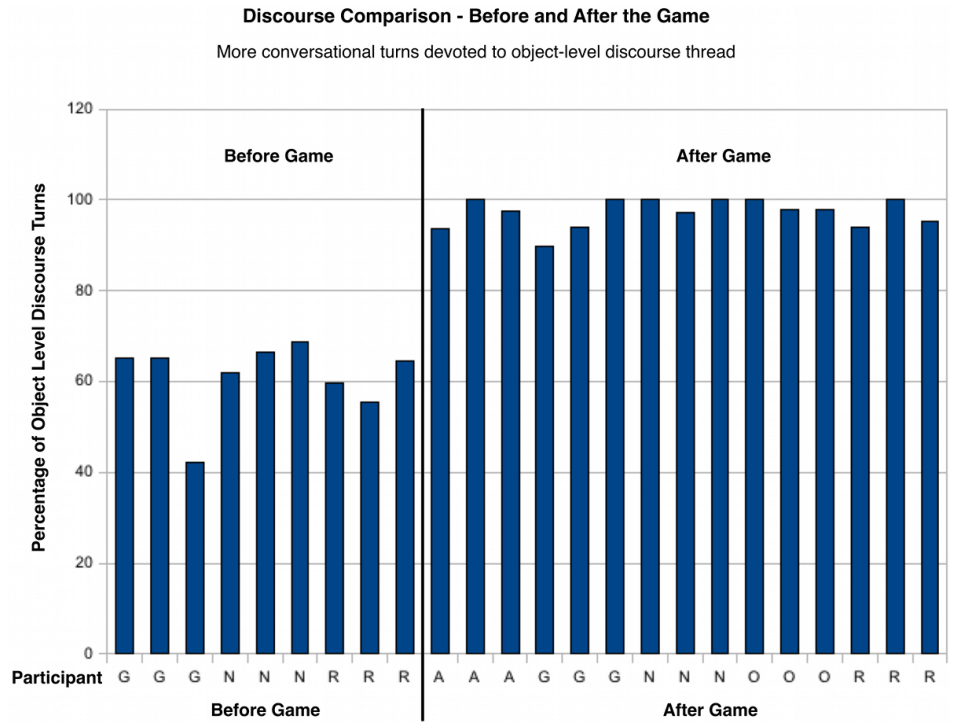

Figure 4.4: Percentage of Conversational Turns Dedicated to Solving the Charade - before and after playing the Mission Impossible Game

\subsubsection{Comparing Charade Game Experience}

Post-questionnaires were conducted verbally after both charade studies. These took the form of a set of statements to which the participants responded on an agreement Likert scale (1-strongly disagree to 5-strongly agree, 3 being no opinion). Statements were kept the same to facilitate the comparison between both studies. Table 4.15 shows a selected set of these questions and the averages of the answers for both first and second charade studies 2. The questions were grouped into five different categories: Comfort (C), Confidence (Cd), Multi-task (MT) and Interaction (I). We shall discuss the data on this table by groups.

By comfort, we mean one being comfortable with both wearing the haptic glove and interacting with the guide through the system. One can see that our participants are much more inclined to wear the glove than to have someone physically holding their hand, as normally happens in traditional instruction. As for confidence, we mean the confidence participants have that the system would bring gains in interactions similar to the charade. In this group, one can observe that after playing the game, participants are more inclined to believe that they would perform better using the system than with human help. We have argued earlier

\footnotetext{
${ }^{2} \mathrm{~A}$ complete listing of the statements used in both charades can be found on page 221
} 
Table 4.15: Blind followers' experience - A comparison between 1st and 2nd charade studies

\begin{tabular}{|c|c|c|c|c|c|}
\hline \multirow[t]{2}{*}{ Group } & \multirow[t]{2}{*}{ Question } & \multicolumn{2}{|c|}{ Charade } & \multirow{2}{*}{$\begin{array}{l}\text { Diff } \\
\%\end{array}$} & \multirow[t]{2}{*}{ Comments on the } \\
\hline & & 1st & 2nd & & \\
\hline Comfort & I felt comfortable using the glove & 4.25 & 4.40 & 3.53 & $\begin{array}{l}\text { 1st and 2nd studies (all } \\
\text { agreed) }\end{array}$ \\
\hline Comfort & $\begin{array}{l}\text { I'd rather use this glove than have } \\
\text { someone physically move my hand to } \\
\text { the letter }\end{array}$ & 3.75 & 4.60 & 22.67 & $\begin{array}{l}1 \text { disagreed in each } \\
\text { study }\end{array}$ \\
\hline Confidence & $\begin{array}{l}\text { If I had someone physically holding } \\
\text { my hand and putting it over the doc- } \\
\text { ument, I would have performed bet- } \\
\text { ter }\end{array}$ & 3.75 & 2.80 & -25.33 & $\begin{array}{l}1 \text { disagreed in each } \\
\text { study }\end{array}$ \\
\hline Confidence & I would perform better with practice & 5.00 & 4.60 & -8.00 & $\begin{array}{l}1 \text { disagreed in each } \\
\text { study }\end{array}$ \\
\hline Confidence & $\begin{array}{l}\text { I would like to participate in future } \\
\text { experiments because I believe this } \\
\text { technology will help students who are } \\
\text { blind }\end{array}$ & 5.00 & 4.80 & -4.00 & $\begin{array}{l}1 \text { st and 2nd } \\
\text { agreed) }\end{array}$ \\
\hline Multi-task & $\begin{array}{l}\text { I could perfectly listen to the guide } \\
\text { while using the glove }\end{array}$ & 4.50 & 5.00 & 11.11 & $\begin{array}{ll}1 \text { st (all but } & \text { agreed), } \\
\text { agrd } \\
\text { agreed) }\end{array}$ \\
\hline Multi-task & $\begin{array}{l}\text { Using the system did not interfere on } \\
\text { my thinking of the solution }\end{array}$ & 3.20 & 3.80 & 18.75 & $\begin{array}{l}1 \text { disagreed in each } \\
\text { study }\end{array}$ \\
\hline Interaction & $\begin{array}{l}\text { The conversation between the guide } \\
\text { and myself flowed naturally }\end{array}$ & 4.25 & 4.80 & 12.94 & $\begin{array}{ll}1 \text { st (all } & \text { but } \\
\text { agreed), } & \text { 2nd } \\
\text { agreed) }\end{array}$ \\
\hline Interaction & $\begin{array}{l}\text { I was able to point at my chart and } \\
\text { ask questions }\end{array}$ & 4.25 & 4.60 & 8.24 & $\begin{array}{l}1 \text { disagreed in each } \\
\text { study }\end{array}$ \\
\hline Interaction & $\begin{array}{l}\text { I used pointing to reduce misunder- } \\
\text { standing in what I said }\end{array}$ & 3.25 & 4.00 & 23.08 & $\begin{array}{l}1 \text { disagreed in each } \\
\text { study }\end{array}$ \\
\hline Interaction & $\begin{array}{l}\text { Because of the system, I perceived } \\
\text { that my communication was better } \\
\text { understood }\end{array}$ & 4.00 & 4.40 & 10.00 & $\begin{array}{ll}1 \text { st (all but } \\
\text { agreed), } & \text { 2nd } \\
\text { agreed) } & \end{array}$ \\
\hline
\end{tabular}

on the multi-modal, multi-task demands of a teacher/student-like interaction. In this group, numbers have also improved. When it comes to interaction, one can observe that our blind participants perceived that: 1) the interaction with the guide flowed more naturally, 2) they could also benefit from pointing, 3) the system helps the conversants.

Together, these results show that there is indeed transference in skill from our skill training game to the phrase charade discourse-oriented game. 


\subsection{Transition}

We posited that the use of the assistive technology must become fully embodied and automatic before it can support dynamic fluent instructional discourse. We advanced the idea of employing a skill training game, to encourage the development of the level of skill necessary. We employed principles of GameFlow theory to develop the game, and to test it in a selfregulated game arcade format. The results of our skill training game studies suggest that this strategy is effective in encouraging skill development within a framework of fun. The game kept our participants motivated, and the manipulation of the level of challenge in the form of game levels scaffolded the development of embodied skill in the use of the assistive technology.

We performed a second phrase charade to determine if the skill acquired by our skill training game would transfer into a discourse environment. The results of our second charade study show a more than two-fold increase in speed over our first charade study before the skill training game. Furthermore, commitment of discourse to discussing the technology practically disappeared. The assistive technology was no longer the focus, and the problem at hand (the charade) and the dynamics of regular discourse maintenance dominated. Our results also showed a strong increase in confidence in the assistive technology and the comfort level in the use of the technology was markedly increased.

In the next chapter, we finally test the system in the scenario for which it was built: Mathematics and science instruction. The studies discussed here show how we prudently advanced towards our ultimate research objective. We tried to investigate all the relevant aspects of the proposed technology in tasks and scenarios increasingly similar to the real instruction. We reacted to the findings by either evolving our system/device or proposing ways for our target user community to adapt to the issues that could not be changed. We therefore believe that the right conditions were created for us to take the next step. 

Chapter 5

On to Instruction 



\section{$5.1 \quad$ Introduction}

In the last chapter, we posited that the SBVI attention to the signals from the haptic glove should recede into the background, so that her conscious attentional and cognitive resources could be dedicated to the task of multimodal comprehension and discourse maintenance. That was accomplished through extensive training made possible by the challenging activity our game provided. However, it is important to note that apart from training the SBVI, both the system as a whole and the haptic device were improved. A much more stable tracking algorithm was implemented and new glove models were introduced. This new glove conforms better with the palm as the vibrating motors are assembled over a foam cushion. Three different (small, medium and large) gloves were made available for better fit different hand sizes. Sturdier velcro straps help the user to adjust the glove for better signal salience and comfort.

At this point, we had a population of participants who were trained on the use of the technology, and who are able to use the system in a deixis-laden dialog. However, instruction discourse is different from a regular day to day conversation. The rate of new higher level information being transfered from instructor to student is much higher. Besides the student has to immediately use this new information to understand what is being conveyed. This imposes challenges and questions that can only be fully understood if we test the system in real instruction.

We understand that our solution can be used to promote inclusive classrooms, where people with disabilities attend mainstream classes. It has been argued that inclusive classrooms are beneficial for both disabled 64] and non-disabled students [254]. Furthermore, such inclusive instruction is required by law Disabilities Education Act Amendments (IDEA, 1997), and the No Child Left Behind Act (NCLB, 2001). A court ruling [263] had also reinforced the non-seggregational approach. Therefore, the discussion is not if inclusive classrooms are good or not, it is how to make them work. The reduction of the gap between special and regular students requires both inclusion of those with special-needs and effective educational methods for all students [15]. One of the most promising practices for helping students with disabilities succeed in the classroom is the use of technology [14].

Taking our system to inclusive classrooms also brings another set of challenges. The most important one is for the instructor who will have to focus both on the SBVI and on the non-impaired students. For that, the speed of interaction and the capacity of our technology to assist communication between a seeing instructor and SBVI are critical. We understand that the instructor's display will help the instructor on regulating the pace of the lecture considering both groups. At this point, we had the following research questions:

- What is the effect of the system on:

- the relationship between image and discourse? 
- the fluency of the lectures?

- learning?

As corollary questions, we had:

- How do students and instructions react to the technology?

- What new challenges in instruction arise from use of the technology?

In the following sections we will present: 1) our experiment design; 2) the analysis instruments followed by data analysis; 3) conclusions; 5) new research opportunities and 6) a summary of the work completed.

\subsection{Experiment Design}

Inclusive classes were setup with three students who have normal sight along with one SBVI. All-sighted status quo control classes were also setup. All student participants (SBVI or no) attended two mathematics mini-courses (A and $\mathrm{B}$ ). Those mini-courses were taught by two instructors. One instructor, who we will call $\mathbf{A}$, taught curriculum A while the other, called $\mathbf{S}$, taught curriculum B. Curriculum A is on planar geometry and curriculum B is about trigonometry. These topics were chosen because of their inherent-visual nature. Both courses had three sections. These sessions build on one another. The lessons' plans for curriculum A can be found on page 235 and those of curriculum B, on page 243.

All student participants were required to take oral exams both before and after attending to classes. Pre and post-tests for both curricula are listed on pages 241 and 242 (curriculum A); and 251 and 252 (curriculum B).

The student participants were divided into seven groups. In the first two groups, the control groups, we had those who attended to all sighted classrooms (curricula A and B). We then divided the remainder students into five groups comprised of one SBVI and three with normal sight. All five groups took both curricula either with or without the system. In a counterbalanced design, the groups who attended curriculum A with the system took curriculum B without it; while those who attended curriculum A without the system, took curriculum B classes with the system. Table 5.1 details how the participants are grouped. 
Table 5.1: Instruction Experiment

\begin{tabular}{|c|l|l|}
\hline Experimental Condition & Curr A(T1) & Curr B(T2) \\
\hline All Sighted & $\mathrm{g} 1(\mathrm{~s} 16, \mathrm{~s} 17, \mathrm{~s} 18, \mathrm{~s} 19)$ & $\mathrm{g} 2(\mathrm{~s} 20, \mathrm{~s} 21, \mathrm{~s} 22, \mathrm{~s} 23)$ \\
\hline Blind w/ System & $\mathrm{g} 3(\mathrm{~b} 1, \mathrm{~s} 1, \mathrm{~s} 2, \mathrm{~s} 3)$ & $\mathrm{g} 5(\mathrm{~b} 3, \mathrm{~s} 7, \mathrm{~s} 8, \mathrm{~s} 9)$ \\
& $\mathrm{g} 4(\mathrm{~b} 2, \mathrm{~s} 4, \mathrm{~s} 5, \mathrm{~s} 6)$ & $\mathrm{g} 6(\mathrm{~b} 4, \mathrm{~s} 10, \mathrm{~s} 11, \mathrm{~s} 12)$ \\
& & $\mathrm{g} 7(\mathrm{~b} 5, \mathrm{~s} 13, \mathrm{~s} 14, \mathrm{~s} 15)$ \\
\hline Blind w/o System & $\mathrm{g} 5(\mathrm{~b} 3, \mathrm{~s} 7, \mathrm{~s} 8, \mathrm{~s} 9)$ & $\mathrm{g} 3(\mathrm{~b} 1, \mathrm{~s} 1, \mathrm{~s} 2, \mathrm{~s} 3)$ \\
& $\mathrm{g} 6(\mathrm{~b} 4, \mathrm{~s} 10, \mathrm{~s} 11, \mathrm{~s} 12)$ & $\mathrm{g} 4(\mathrm{~b} 2, \mathrm{~s} 4, \mathrm{~s} 5, \mathrm{~s} 6)$ \\
& $\mathrm{g} 7(\mathrm{~b} 5, \mathrm{~s} 13, \mathrm{~s} 14, \mathrm{~s} 15)$ & \\
\hline
\end{tabular}

Legend:

1. Cur A - Curriculum A, Cur B - Curriculum B.

2. T1 and T2 - Teacher 1 and teacher 2 .

3. g1...g7: 7 groups.

4. s1...s23: 23 sighted students.

5. b1...b5: 5 SBVI

\subsubsection{The Participants}

All student participants were from Wright State University. None of them, including those who are blind, were majoring on math-related fields. Both curricula use concepts which were supposedly learned in high school. Consequently, it is likely that many participants had already forgotten several of those concepts.

Our 5 SVBI participants were the same that participated in the second charade study. It is important to introduce the age at which they became blind because previous visual experience effects learning positively [64]. Table 5.2 shows the age at which each of the participating SBVI became blind.

Curriculum A instructor, A was a male mid-twenties mathematics graduate student who wants to become mathematics high school teacher but had no real world teaching experience. Curriculum B instructor, $\mathbf{S}$, was a late forties female professional high school mathematics teacher for almost 30 years who never taught a student who is blind. 
Table 5.2: SBVI participants and when they became blind

\begin{tabular}{|cr|}
\hline SBVI & Blindness onset (age) \\
\hline N & 16 \\
G & 13 \\
O & 2 \\
R & From birth \\
A & 12 \\
\hline
\end{tabular}

\subsection{Data Analysis}

\subsubsection{Challenges}

\section{Too small pool of SBVI}

We conducted our studies at Wright State University (WSU) where there is a concentration of students with a wide range of disabilities and where these students are integrated into the general student population. This environment is somewhat unique because of the low percentage of students with disabilities who attend college. Only a very small number of high school graduates who are blind go to college [252]. Furthermore, solely those who were trained with the glove were qualified to participate in our instruction study.

\section{Too high variance of prior knowledge}

Despite the efforts to recruit only those who were majoring on fields not related to mathematics, we were still vulnerable to high variance of prior knowledge. The factor is more relevant among the SBVI. As we stated before, the age at which they became blind plays an important role on learning. Furthermore, we made no restriction to what year the students were in their undergraduate program. One can argue that freshmen would have advantage since the curricula covered high school concepts which they might have seen more recently than seniors.

\section{Different curricula and Instructor's background}

We had only two instructors teaching different curricula and with very different backgrounds. This makes it very hard to make between curricula comparisons. 


\subsubsection{Analysis Instruments}

In this section, we first introduce our analysis instruments and discuss how they will be used to help in answering our research questions. Later, we take a second and more in depth view on each of them as we present the detailed data analysis. Finally, we present a summary of the instruments highlighting the ones statistically significant.

\section{Discourse and Situated Analysis}

This instrument should help us answering the research question: What is the effect of the system in the relationship between image and discourse?

We will try to answer this question with two distinct psycholinguistic analyses. In the first, we will use McNeill and Quek's concepts of Growth point [190] pp 81-82) and Hyperphrase [217] to understand how the system effects gesture and speech synchrony and how it impacts the creation of learning opportunities for the SBVI. This first analysis is detailed on page 119. In the second analysis, we will use Clark's Common Ground theory ([51], p. 4) to understand how instructors and students used evidence ([51, p. 37) and assumption ([51, p. 39) of mutual understanding with and without the system. This second analysis is presented in depth on page 123 .

For these two analysis, we used time-aligned transcribed video obtained from the trials. All trials were recorded by three cameras capturing the interaction from three different vantage points (instructor view, SBVI view and whole class view). The interaction was transcribed, speakers identified and the duration of the conversational turns recorded. Videos were synchronized with each other and with the transcriptions. For each trial, a MacVisSTA [227] project was created to hold its correspondent data (synched videos and transcriptions). Relevant observations were annotated on each MacVisSTA project.

\section{Lecture Fluidity Analysis}

In this analysis, we try to answer the research question: What is the effect of the system on the fluency of lectures? For this, we propose the list of measures shown on table 5.3. The table also points to where the detailed analysis on each measure can be found.

All the detailed analysis were performed in a similar way. Since we were looking for differences that might be attributed to the use of the system, we first compared the measures obtained from the trials where the system was employed against those where it is not used. We call this the overall analysis. There, we ran one-way ANOVA at 90\% confidence interval (CI), instead of the traditional 95\%. The lower CI is motivated by the fact that we had only six lessons (3 per curriculum) and lowering the CI would also decrease the likelihood of committing the type II error in our statistical analysis. 
Other factors besides the use of the system or not might also have influenced the measures. Examples of such factors are: Instructor and what is covered on each lesson. To mitigate these factors, we performed a second analysis, where the measures from each instructor/lesson with the system were paired with its "without the system" correspondent. Once the pairs were formed, we ran a paired Student's $t$ also at $90 \%$ CI.

We anticipate that we would only find significant differences from the the first analysis in cases where the system alone had a very strong influence on the measures. Other than that, only trends are observerable. This is why we call the first analysis, the overall analysis. It is from the second one, the paired lessons analysis, that we expect to draw more definitive conclusions.

Table 5.3: Lecture Fluency Measures

\begin{tabular}{|c|c|c|}
\hline Measure & Rationale & How it was calculated \\
\hline $\begin{array}{l}\text { Number of words } \\
\text { per conversational } \\
\text { turn (Instructor) - } \\
\text { WPT }\end{array}$ & $\begin{array}{l}\text { Pointing adds precision to } \\
\text { utterance and make them } \\
\text { shorter. We compared the } \\
\text { mean number of words per } \\
\text { utterance in the instructor's } \\
\text { speech with and without } \\
\text { the system }\end{array}$ & $\begin{array}{l}\text { Total words uttered by the instructor } \\
\text { per lesson divided by the number of } \\
\text { her conversational turns in one lesson } \\
\text { in one experimental condition was } \\
\text { compared to the same lesson from the } \\
\text { same curriculum in the other experi- } \\
\text { mental conditions - Repetitions were } \\
\text { averaged. Detailed analysis on page } \\
\text { 135. }\end{array}$ \\
\hline $\begin{array}{l}\text { Mean duration } \\
\text { of instructor's } \\
\text { conversational } \\
\text { turns - DIT }\end{array}$ & $\begin{array}{l}\text { When using the system, in- } \\
\text { structors have to hold their } \\
\text { pointing position until the } \\
\text { student reaches it. We as- } \\
\text { sessed the impact of the } \\
\text { navigation time on the du- } \\
\text { ration of the instructor's } \\
\text { turns }\end{array}$ & $\begin{array}{l}\text { Each conversational turn was tran- } \\
\text { scribed along with the time it started } \\
\text { and the time it ended. Times were } \\
\text { obtained from the tapes. We aver- } \\
\text { aged the duration of each instruc- } \\
\text { tor turn (in seconds) in one lesson in } \\
\text { one experimental condition and com- } \\
\text { pared to the same lesson in the same } \\
\text { curriculum in the other experimen- } \\
\text { tal condition - Repetitions were aver- } \\
\text { aged. Detailed analysis on page } 136 .\end{array}$ \\
\hline & & ued on next page \\
\hline
\end{tabular}




\begin{tabular}{|c|c|c|}
\hline \multicolumn{3}{|r|}{ continued from previous page } \\
\hline Measure & Rationale & How it was calculated \\
\hline $\begin{array}{l}\text { Mean number of } \\
\text { hand positioning } \\
\text { events }- \text { HPE }\end{array}$ & $\begin{array}{l}\text { When teaching the blind, } \\
\text { instructors normally have } \\
\text { to hold the students hand } \\
\text { and help her explore im- } \\
\text { ages. In inclusive class- } \\
\text { rooms, this disrupts the lec- } \\
\text { ture, brings unwanted at- } \\
\text { tention to the blind and el- } \\
\text { evates the risk of stigma- } \\
\text { tization. We investigate } \\
\text { the system's impacts on } \\
\text { number of hand positioning } \\
\text { events }\end{array}$ & $\begin{array}{l}\text { We counted the times the instructor } \\
\text { stopped the lesson, walked up to the } \\
\text { SBVI and physically repositioned her } \\
\text { hand. The events were averaged by } \\
\text { lesson and then averaged by curricu- } \\
\text { lum and experimental condition. De- } \\
\text { tailed analysis on page } 138 \text {. }\end{array}$ \\
\hline $\begin{array}{l}\text { Percentage of con- } \\
\text { versational turns } \\
\text { aimed at lesson's } \\
\text { objectives TLO }\end{array}$ & $\begin{array}{l}\text { When a new human-to- } \\
\text { human mediating technol- } \\
\text { ogy is introduced there } \\
\text { is the risk of "attentional } \\
\text { shift". We wondered if the } \\
\text { the system made instruc- } \\
\text { tors and students less fo- } \\
\text { cused on the lessons' objec- } \\
\text { tives. }\end{array}$ & $\begin{array}{l}\text { We classified each conversational } \\
\text { turn (instructor and students) as be- } \\
\text { longing to either one of the three } \\
\text { discourse levels: Object level, Meta } \\
\text { level and Para level [191]. In the } \\
\text { object level, instructor and students } \\
\text { are engaging on issues related to the } \\
\text { lesson objectives. In meta level, the } \\
\text { subject is the discourse itself. Turns } \\
\text { related to discourse repair were also } \\
\text { coded as meta level. Para level utter- } \\
\text { ances relate to the individual experi- } \\
\text { ence. Words of encouragement like: } \\
\text { "There you go", "take your time", } \\
\text { etc were coded as as belonging to the } \\
\text { para level thread. We report the per- } \\
\text { centage of object level turn. Detailed } \\
\text { analysis on page } 140 \text {. }\end{array}$ \\
\hline & & continued on next page \\
\hline
\end{tabular}




\begin{tabular}{|c|c|c|}
\hline \multicolumn{3}{|r|}{ continued from previous page } \\
\hline Measure & Rationale & How it was calculated \\
\hline $\begin{array}{l}\text { Percentage of De- } \\
\text { ictic Expressions - } \\
\text { DE }\end{array}$ & $\begin{array}{l}\text { With this measure we can } \\
\text { assess the impact of the sys- } \\
\text { tem on the creation of deic- } \\
\text { tic opportunities in the in- } \\
\text { structor's discourse. It is } \\
\text { important to acknowledge } \\
\text { that, as we showed in chap- } \\
\text { ter one, not all pointing acts } \\
\text { are accompanied by deic- } \\
\text { tic expressions. However, } \\
\text { we argue that the measure } \\
\text { is still valid because of the } \\
\text { paired comparisons. }\end{array}$ & $\begin{array}{l}\text { Each conversational turn was au- } \\
\text { tomatically parsed for words like: } \\
\text { here, there, that. On the A sec- } \\
\text { ond and manual parse assigned those } \\
\text { turns as either containing or not } \\
\text { a deictic expression. The number } \\
\text { showed that represents the percent- } \\
\text { age of turns having deictic expres- } \\
\text { sions (number of turns having de- } \\
\text { ictic expressions/total turns). De- } \\
\text { tailed analysis on page } 142 \text {. }\end{array}$ \\
\hline
\end{tabular}

\section{Instructor and Student Experience}

Upon the completion of both curricula, instructors and students who attended inclusive classrooms responded to Likert-type scale questionnaires. During the interviews, subjects were encouraged to not only give us the number corresponding to their answer, but also elaborate on them. Questions were on lecture fluency, instructional material, attention (including whether they perceived the system as a distraction), engagement, understanding (including the images associated to the concepts), comfort and fatigue. Finally, we were also interested in knowing what were their expectations about the impact of system on their final oral exams.

A complete discussion on this measure can be found on page 145 .

\section{Learning}

This is the hardest analysis on this study. There are too many factors involved and not enough statistical power for a significant conclusion to be drawn. As we stated on section (5.3.1 - challenges), we had only five SBVI taking two different courses taught by two different instructors. For these reasons, this will be our last discussion because we will use the results from the previous sections to fuel a more in-depth debate on this issue. The detailed analysis can be found on page 149 . 


\subsubsection{Detailed Analysis and Measures}

\section{Situated Analysis (Growth Point and Hyperphrase)}

For McNeill ([190] pp 81-82), Growth Points (GPs) are idea units and come from the dynamic combination of linguistic categorical and imagistic components which "live" a period of instability. The resolution of this instability crystallizes an idea unit. GP is normally inferred from the speech-gesture synchrony and co-expressivity. If instructor and student share the same GP, we can say that they inhabit the same state of cognitive being, which is what instructional communication aims. Hyperphrase is a communicative pulse comprised of verbal, gestural and gaze level simultaneously organized thematically around an idea unit [217]. Armed with these concepts along with the time-alined transcribed videos obtained from the trials, we were able to identify three distinct situations where the use of the system impacts (or not) speech/gesture syncrony:

1. Instructor utterance-student deixis synchrony creates conditions for immediate GP;

2. Instructor utterance-student deixis synchrony creates hyperphrase that includes but does not precisely localize the GP;

3. Instructor's utterance stops prematurely and student is unable to form either an appropriate GP or hyperphrase.

We shall now present examples of these situations. 
1. Instructor utterance-student deixis synchrony creates conditions for immediate GP. Example:

\begin{tabular}{|ll|}
\hline Instructor & Observations \\
"Right here - & The instructor's pointing meets intersection \\
when the sun- & of Line A and Line $\mathrm{C}$ at this utterance \\
light meets & point. His focal idea of GP is the angle \\
the stick as & of sunlight (pointing in the graph) with re- \\
its coming & spect to the surface of the Earth (in speech). \\
out from the & These together are a thematic unit. The \\
Earth" & student, however, is not at the Line A-Line \\
& C intersection but she is receiving signals \\
& from the glove and starts moving her hand \\
& along her graphic line toward the intersec- \\
& tion. \\
"It meets at & $\begin{array}{l}\text { Student's reading (blue dot) reaches the } \\
\text { angle" A - Line C intersection just as the in- } \\
\text { structor says "angle". She can then form an } \\
\text { idea unit or GP with her deixis with this } \\
\text { word, which is the same idea unit the in- } \\
\text { structor had focused on. So here is a case } \\
\text { where the student, thanks to timing, and } \\
\text { despite of inevitable delay, can directly ap- } \\
\text { prehend the core idea. }\end{array}$ \\
\end{tabular}


2. Instructor utterance-student deixis synchrony creates hyperphrase that includes but does not precisely localize the GP. Example:

\begin{tabular}{|ll|}
\hline Instructor & Observations \\
"And then & Instructor's wand moves up the line on his graph. \\
this parallel & His GP or core idea here highlights the paral- \\
line is also lelism of the line (rather than any other prop- & the sunlight \\
erty). The student, again, is not at this line but \\
hitting the & begins to move up to the line on her graphic. As \\
Earth" & she reaches it, the instructor is saying: \\
& \\
"And so" & In this instance, however, her deixis and the in- \\
& structor's speech do not form the same GP. There \\
& would be, for her, a GP comprised of deixis and \\
& this connective from the utterance: something \\
& like, at this moment, there is a link being forged \\
& from something before, which is the condition \\
& for something yet to come. Blind listeners are \\
& acutely sensitive to linguistic cues, and there is \\
& a good chance the student would pick this kind \\
& of idea unit. Although not the instructor's GP, \\
& it also links back to the instructor's utterance (it \\
& is the condition the "and so" is relating to the \\
& next as yet unspoken step), and this is the hy- \\
& perphrase that bundles the student's deixis, the \\
& instructor's speech, including the entire stretch \\
& that included his original focal idea. So, this \\
& hyperphrase route also makes information avail- \\
& able, albeit less precisely focused.
\end{tabular}


3. Instructor's utterance stops prematurely and student is unable to form either an appropriate GP nor hyperphrase. Example:

\begin{tabular}{|c|c|c|}
\hline Instructor & Observations & Scene \\
\hline $\begin{array}{l}\text { "My stick is } \\
5 f t "\end{array}$ & $\begin{array}{l}\text { The instructor points at the side of the rectangle } \\
\text { corresponding to the stick. The student starts } \\
\text { moving toward the teacher's pointing. Before she } \\
\text { gets to the side of the rectangle that corresponds } \\
\text { to the stick, the instructor moves to another side } \\
\text { of the triangle and says: }\end{array}$ & \\
\hline $\begin{array}{lr}\text { "and } & \text { my } \\
\text { shadow } & \text { is } \\
10 " & \end{array}$ & $\begin{array}{l}\text { The instructor introduced a new idea (the } \\
\text { shadow), before the student had the opportunity } \\
\text { to apprehend the previous idea (the stick). This } \\
\text { confounded the student because she is unable to } \\
\text { form either an appropriate GP or hyperphase. }\end{array}$ & \\
\hline
\end{tabular}




\section{Situated Analysis (Common Ground)}

For Clark "All language use rests on a foundation of information that is shared by the participants, what is technically called their common ground" ([51], p. 4). According to him, speakers try to convey information that they think listeners already have or can understand. Key to the present analysis is Clark's observation that "people's mental representations of mutual knowledge are inferences based on certain evidence and assumptions, (Clark [51], p. 5). Clark defines the Immediate Physical Co-presence (IPC) is the strongest evidence possible. The following exchange illustrates how instructor and students can engage in IPC.

- Instructor says: "This line" and points at the line.

- Students look at the line.

- Instructor sees that the student are looking at the line.

Pointing gestures help the parties to establish IPC because it simultaneously provides physical evidence to both instructor and students about the object in discussion (the line). If there is a SBVI in the audience, she would not have engaged in IPC with the instructor. She will not have physical (or perceptual) evidence of the instructor's pointing. In this case, the instructor has two options. First, she could change her behavior to engage the SBVI in IPC (possibly holding the SBVI's hand and directing it to the line). Second, she could assume that the SBVI has enough previous information to identify the line.

One important instance of assumption is the Locatability Assumption (LA) (Clark [51], p. 39). The speaker assumes that the listener can discover the referent ("this line") and bring it to "view" simultaneously to her. According to Clark's Presentation/Acceptance model (Clark [51], pp 151-173), utterance presentations can either be accepted (understood) or not. Unaccepted utterances need to be repaired before they snowball (Clark [51], p 164), consequently, harming concept conveyance.

We analyzed concept conveyance across experimental conditions in terms of the number of evidences and assumptions and their impact on utterance acceptance. Our analysis was confined to utterances that involve presentation or references to graphical elements in the instruction material. We used MacVisSTA [227] to identify the occurrences of immediate physical co-presence (IPC) and linguistic assumptions (LA). Each occurrence of IPC or LA was assigned a final state. The final state is always related with listeners ability to resolve the referent and consequently, accept the utterance. Its value is assigned, after watching the videos and judging if the listener had a real chance to understand the speaker. Possible final state values are: $\mathrm{R}$ - Resolved, $\mathrm{U}$ - Unresolved, $\mathrm{P}$ - probably resolved. P - Probably status is assigned when speaker did not do the "final check" on the listener's understanding and the coder is not sure if the listener really understood. We shall now present our analysis on the cases where the same concept was conveyed by the same instructor across experimental conditions. 
First Case: Concept - Vertical Angles. Definition: When angles are formed from two intersecting lines and are not adjacent (Figure 5.1). They all share a vertex. Such angles are congruent and thus are equal in measure, Euclid [73, Proposition I:15. The table below

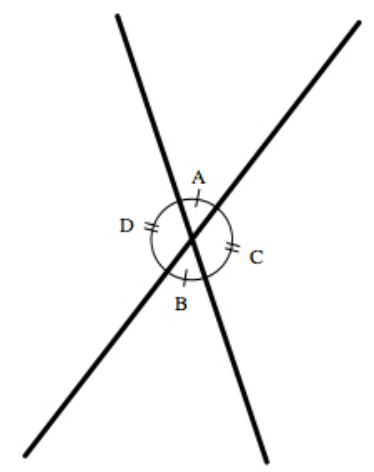

Figure 5.1: $\mathrm{A}$ and $\mathrm{B}$ has the same measure. $\mathrm{C}$ and $\mathrm{D}$ also has the same measure

shows how this concept was conveyed by the same instructor in all three experimental conditions. Each column in that table shows the instructor/student interaction in one particular condition: classes with only sighted students, inclusive classroom where the system was used and inclusive classrooms where the system was not used.

\begin{tabular}{|l|l|l|}
\hline \multicolumn{3}{|c|}{ Vertical Angles } \\
\hline $\begin{array}{l}\text { Anstructor: "If we have A, like } \\
\text { this is A" }\end{array}$ & $\begin{array}{l}\text { With the system } \\
\text { two lines cross, we have T" }\end{array}$ & $\begin{array}{l}\text { Without the system } \\
\text { looking at where the vertical line } \\
\text { meets the horizontal line on our } \\
\text { graph" }\end{array}$ \\
\hline
\end{tabular}




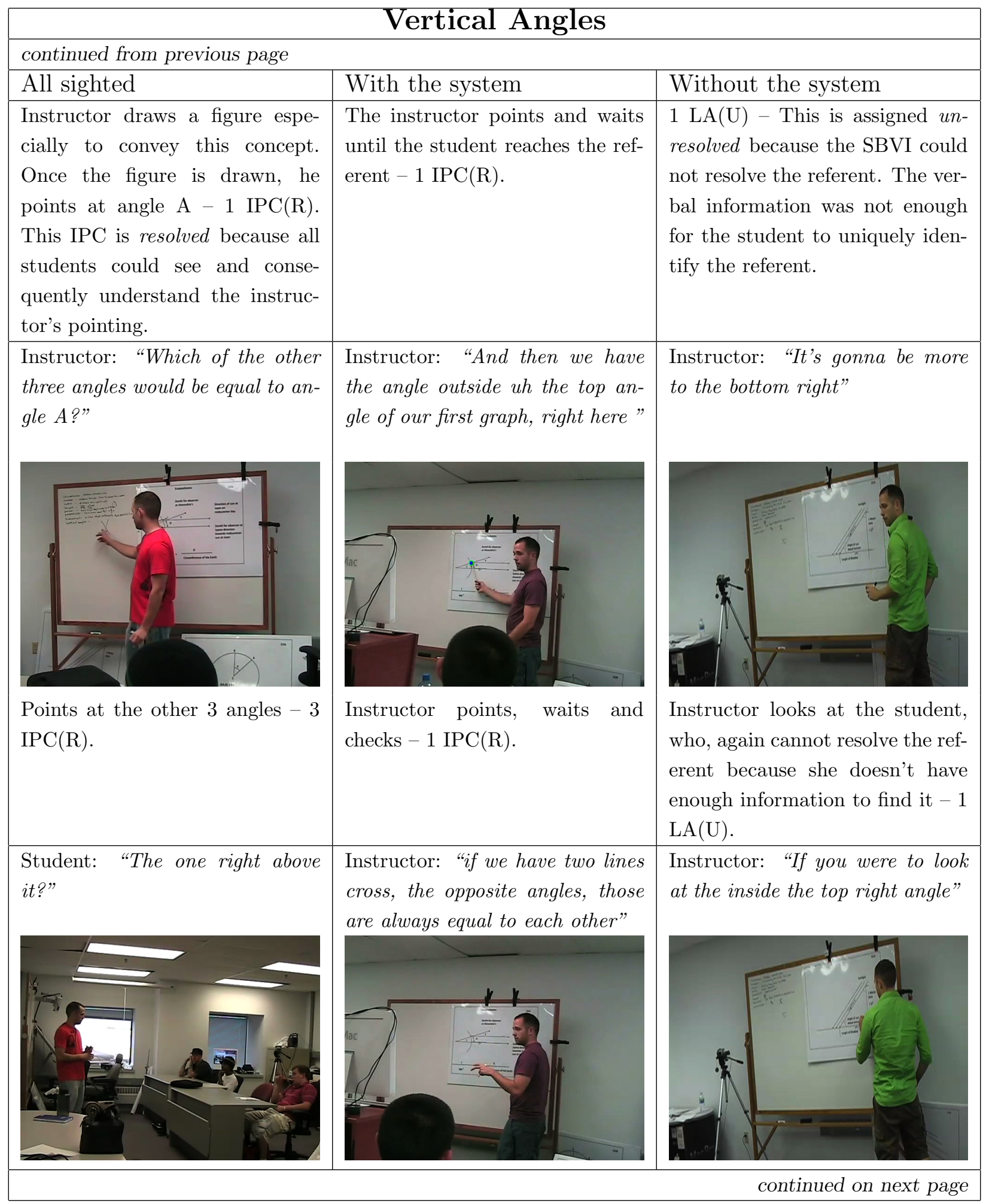




\begin{tabular}{|c|c|c|}
\hline \multicolumn{3}{|c|}{ Vertical Angles } \\
\hline \multicolumn{3}{|l|}{ continued from previous page } \\
\hline All sighted & With the system & Without the system \\
\hline \multirow[t]{5}{*}{$\begin{array}{l}\text { Student points at the board }-1 \\
\text { IPC(A) - It is coded as assumed } \\
\text { because it is impossible for the } \\
\text { instructor to resolve the pointing } \\
\text { due to his vantage point. }\end{array}$} & $\begin{array}{l}\text { Once the instructor had gathered } \\
\text { enough evidence that the blind } \\
\text { has the appropriate imagery, he } \\
\text { can verbalize the concept defini- } \\
\text { tion. }\end{array}$ & $\begin{array}{l}\text { The instructor tries to give more } \\
\text { information of where to find the } \\
\text { referent. However, he never } \\
\text { checks if the SBVI was able to } \\
\text { successfully find it. He just as- } \\
\text { sumed that she was able to find - } \\
1 \text { LAU. }\end{array}$ \\
\hline & & $\begin{array}{l}\text { Instructor: "If we were to label } \\
\text { that } A \text { " }\end{array}$ \\
\hline & & $\begin{array}{l}\text { Instructor writes the letter } \mathrm{A} \\
\text { on the board }-1 \operatorname{IPC}(\mathrm{U}) \text {. }\end{array}$ \\
\hline & & $\begin{array}{l}\text { Instructor: "The vertical angle to } \\
\text { that would be the bottom right an- } \\
\text { gle" }\end{array}$ \\
\hline & & $\begin{array}{l}\text { Another locatability assump- } \\
\text { tion that cannot be resolved } \\
\text { by the student }-1 \mathrm{LA}(\mathrm{U}) \text {. In- } \\
\text { structor knows that the stu- } \\
\text { dent is falling behind and } \\
\text { shows signs of frustration. }\end{array}$ \\
\hline \multicolumn{3}{|r|}{ lext page } \\
\hline
\end{tabular}




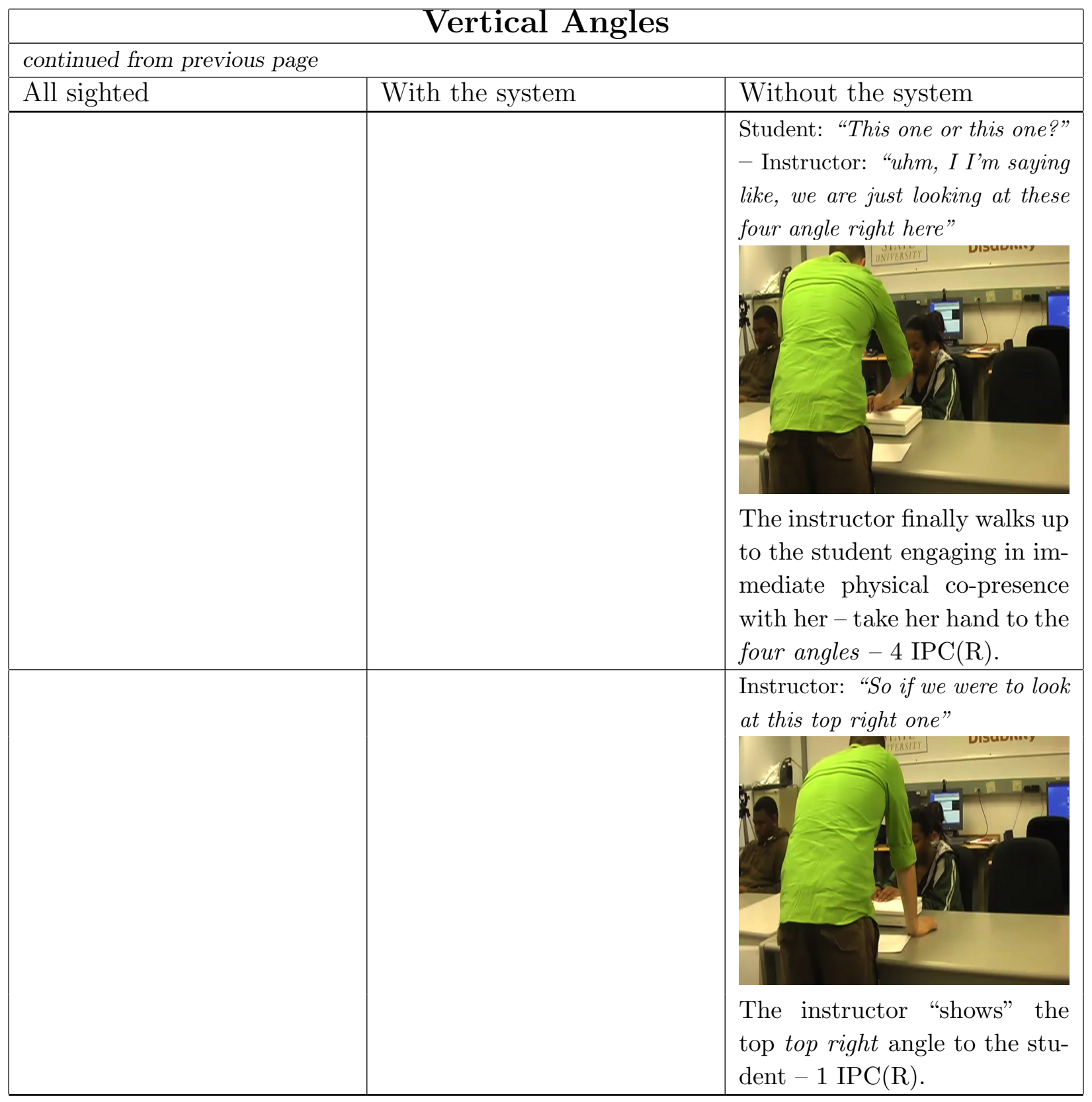


Second Case: Transversal line. Definition: A line that intersects parallel lines forming correspondent congruent (identical) angles, [73], Proposition I:27.

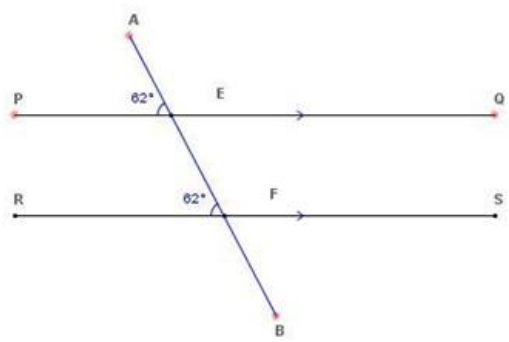

Figure 5.2: Transversal line creates congruent angles when it intersects parallel lines

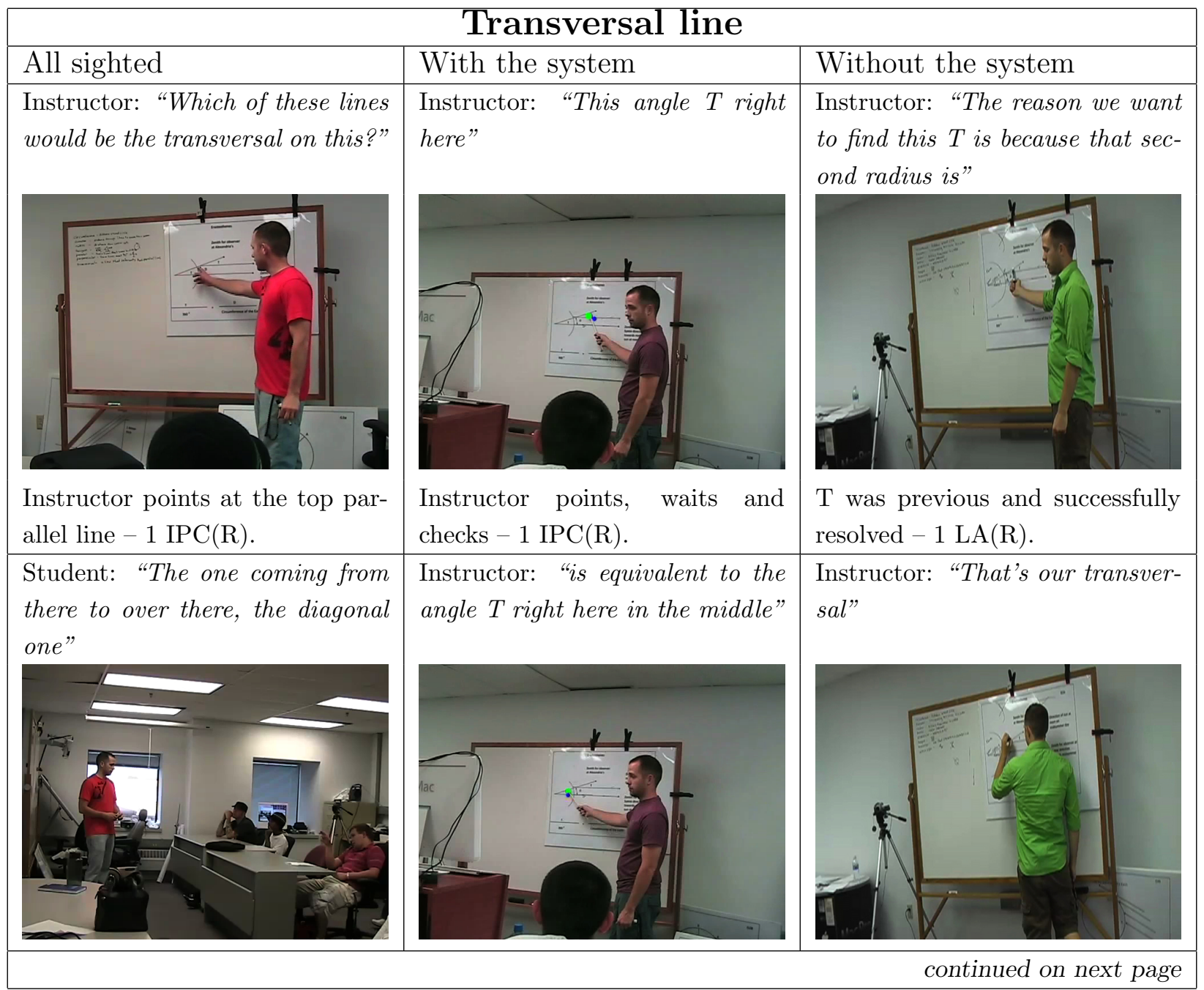




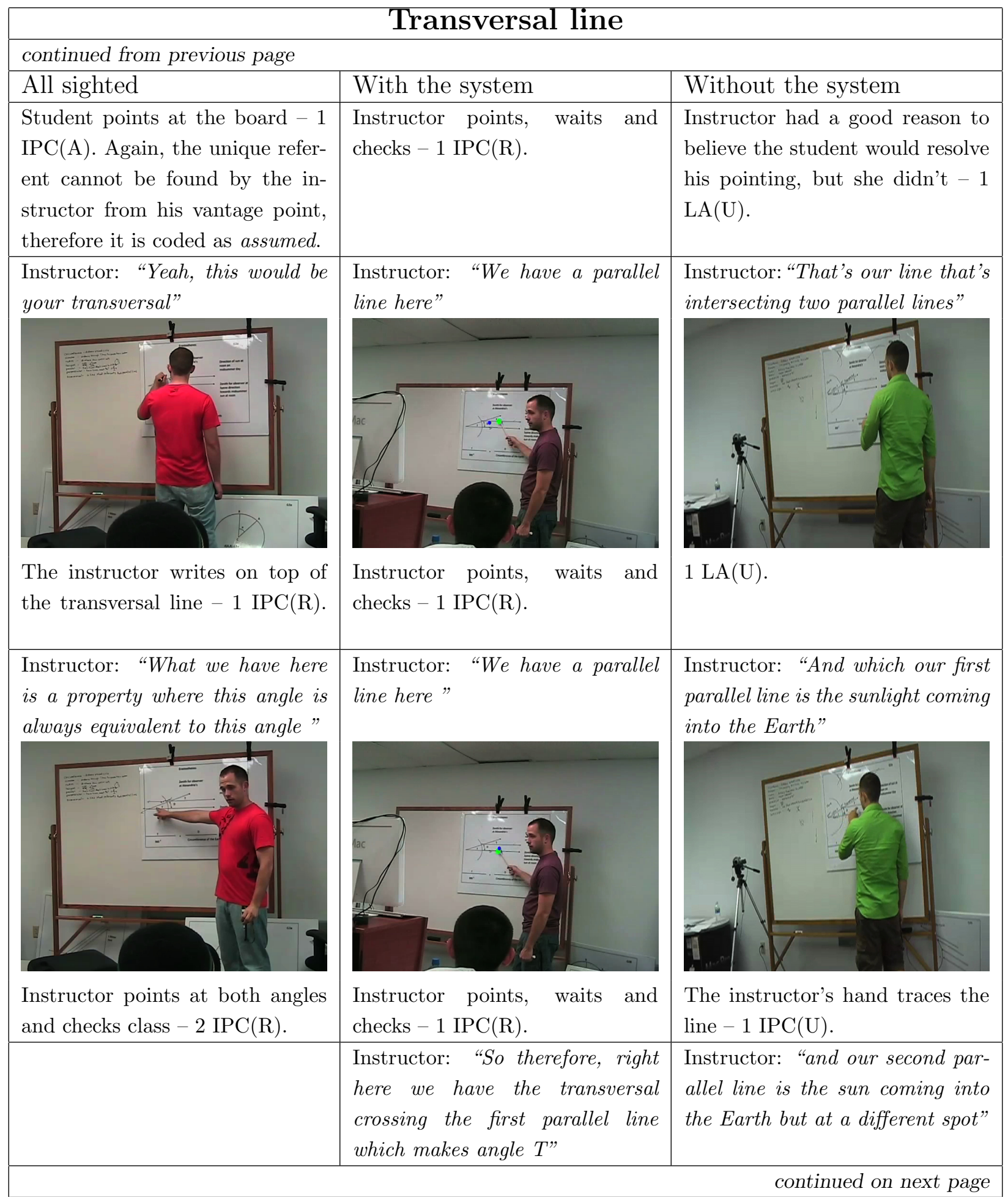




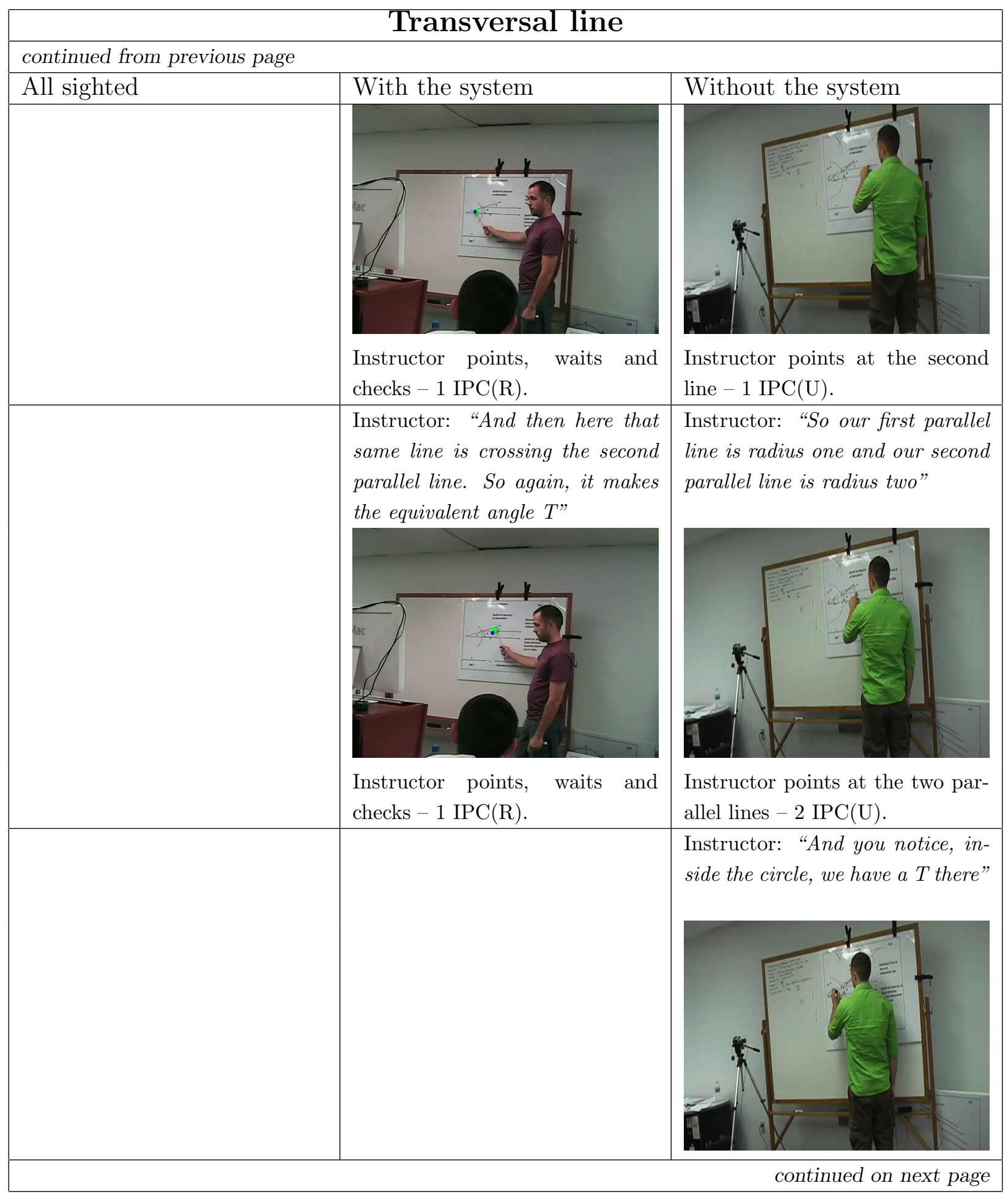




\begin{tabular}{|l|l|l|}
\hline \multicolumn{2}{|c|}{ Transversal line } \\
\hline continued from previous page & With the system & $\begin{array}{l}\text { Without the system } \\
\text { IPC(U). }\end{array}$ \\
\hline All sighted & & $\begin{array}{l}\text { Instructor: "And then outside the } \\
\text { circle, we have a T" }\end{array}$ \\
\hline & & \\
& &
\end{tabular}


Third Case: Diameter. Definition: A diameter of the circle is any straight line drawn through the center and terminated in both directions by the circumference of the circle, and such a straight line also bisects the circle, Euclid [73] Definition I:17.

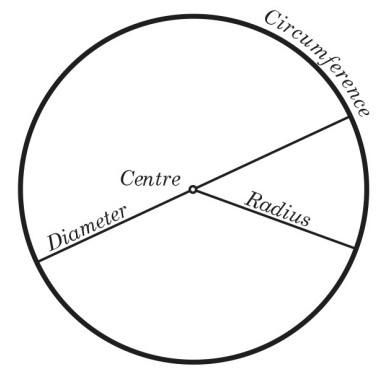

Figure 5.3: A circle with its center, diameter, radius and circumference identified

\begin{tabular}{|l|l|l|}
\hline \multicolumn{3}{|c|}{ Diameter } \\
\hline All sighted & With the system & Without the system \\
\hline $\begin{array}{l}\text { Instructor: "Okay, diameter } \\
\text { measures, how about if I said this: } \\
\text { from one side of the circle to } \\
\text { the other side of the circle going } \\
\text { of the circle to the left edge of the } \\
\text { circle, right through the middle, } \\
\text { that's the diameter." }\end{array}$ & $\begin{array}{l}\text { Instructor: "Is this a diameter?" } \\
\text { the center." }\end{array}$ \\
$\begin{array}{l}\text { Instructor points twice, once at } \\
\text { each edge of a diameter - } 2 \\
\text { IPC(R). }\end{array}$ & $\begin{array}{l}\text { Instructor drifts her wand over } \\
\text { the line representing the diame- } \\
\text { ter, checks if the student was able } \\
\text { to follow her - } 1 \text { IPC(R). }\end{array}$ & $\begin{array}{l}\text { The instructor traverses the circle } \\
\text { with her hand in a straight trajec- } \\
\text { tory that does not pass through } \\
\text { the middle - } 1 \text { IPC(U). }\end{array}$ \\
\hline $\begin{array}{l}\text { Instructor: "So don't think it's } \\
\text { like up here from one side of the } \\
\text { circle to the other side of the cir- } \\
\text { cle. } \\
\text { That that has a different }\end{array}$ & $\begin{array}{l}\text { Instructor: "and thats the key by } \\
\text { the way, right through the center } \\
\text { of the circle" }\end{array}$ & $\begin{array}{l}\text { Instructor: "Okay, and what I did } \\
\text { G }<<\text { laughs }>>\text { what I did, if I'm } \\
\text { not gonna scare you to death, is } \\
\text { like just went across like this" }\end{array}$ \\
\hline
\end{tabular}




\begin{tabular}{|c|c|c|}
\hline \multicolumn{3}{|c|}{ Diameter } \\
\hline \multicolumn{3}{|l|}{ continued from previous page } \\
\hline All sighted & With the system & Without the system \\
\hline & & \\
\hline $\begin{array}{l}\text { points twice showing a chord but } \\
\text { never saying the word (chord) }- \\
2 \operatorname{IPC}(\mathrm{R}) \text {. }\end{array}$ & $\begin{array}{l}\text { Instructor holds her pointing } \\
\text { while conveying the concept. She } \\
\text { waits and checks if the SBVI was } \\
\text { able to move along the line corre- } \\
\text { sponding to the diameter. }\end{array}$ & $\begin{array}{l}\text { Instructor holds the student's } \\
\text { hand and traces the area on his } \\
\text { graph that corresponds the area } \\
\text { she traced on the board }-1 \\
\text { IPC(R). The instructor conveys } \\
\text { the same concept twice: One } \\
\text { for the sighted, another for the } \\
\text { SBVI. Only after she has en- } \\
\text { gaged with immediate physical } \\
\text { co-presence with the blind, she } \\
\text { conveys the concept. }\end{array}$ \\
\hline
\end{tabular}




\section{Situated Analysis (Growth Point/Hypephrase and Common Ground) - Conclu- sions}

We now present our conclusions from the situated analysis. With respect to growth point and hyperphrase, three distinct situations of system use were identified:

1. Instructor utterance-student deixis synchrony creates conditions for immediate GP;

2. Instructor utterance-student deixis synchrony creates hyperphrase that includes but does not precisely localize the GP;

3. Instructor's utterance stops prematurely and student is unable to form either an appropriate GP nor hyperphrase.

We understand that when carefully used, the $H D S$ creates more effective learning opportunities. It provides evidence of image and discourse fusion through pointing, creating conditions for GP/Hyperphrase sharing. However, it can also be frustrating for the SBVI, especially when the instructor does not wait for her. This causes a snowball effect and hinders learning.

With respect to common ground analysis, the use of the system created the conditions for higher number of IPCs because:

1. The instructor's display provided acute evidence to the instructor as whether the student was able to resolve the referent.

2. The haptic glove not only helped the student to navigate to the referent (greatly increasing the chance of its resolution), but also indicated when it has been reached.

These two components (instructor's display and haptic glove) correspond to what Clark calls manifest coordination devices [52]. They create perceptual cues that instructor and SBVI use to coordinate their actions.

The losses in the "without the system" scenario can be compensated if the instructor verbally checks the student understanding. Alternatively, instructors can engage in IPC with the blind by taking her hand and helping her to explore the figure. Furthermore, instructors made verbal references to labels on the figures to help the SBVI finding the relevant portions of the images. This label-aided referent resolution strategy was also observed by Supalo [257].

However, these "compensating" strategies do not come for free. In this scenario utterances demand more time and effort to be produced and understood [53, 163]. In several occasions, the strategies were abandoned as instructors ramped up the pace to cover the lessons objectives, inevitably leaving the SBVI behind. 


\section{Words per conversational turn - WPT (Instructor)}

- How it was measured - Total words uttered by the instructor per lesson divided by the number of her conversational turns in one lesson in one experimental condition was compared to the same lesson in the same curriculum in the other experimental conditions. Repetitions were averaged.

- Overall Comparison - Figure 5.4 and table 5.4 show results obtained from the overall analysis. Albeit statistically inconclusive, they reveal that when using the system, instructors tend to have conversational turns containing roughly the same amount of words that those uttered when they had only sighted students in the audience. In the "without the system" condition, instructors normally use more words to give the SBVI verbal cues of where to go to find relevant points on the figure.

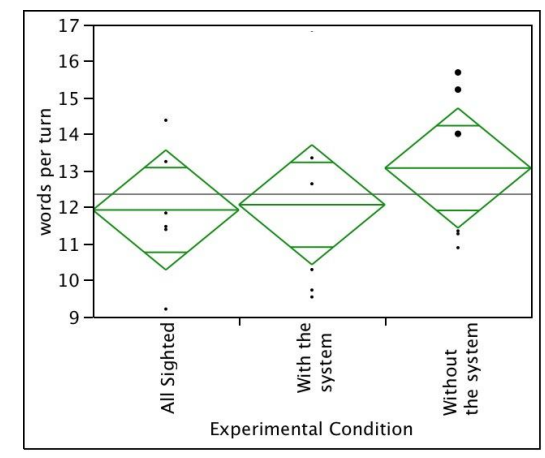

Figure 5.4: Words per conversational turn (Instructor) - Overall Comparison

Table 5.4: Words per conversational turn (Instructor) - Overall comparison

\begin{tabular}{|lllrrr|}
\hline Level & $n$ & Mean & Std Error & Lower 90\% & Upper 90\% \\
\hline All Sighted & 6 & 11.923 & 0.936 & 10.283 & 13.564 \\
With the system & 6 & 12.067 & 0.936 & 10.426 & 13.707 \\
Without the system & 6 & 13.072 & 0.936 & 11.431 & 14.712 \\
\hline
\end{tabular}

- Paired Lessons Analysis - Tables 5.5 and 5.6 show how lessons content and experimental condition affected the number of words the instructor needed in conversational turns. When considering both curricula together, we found that instructors did use fewer words per turn when covering the same material in inclusive classrooms when the system was used as opposed to when it is not $(\mathrm{df}=5, \mathrm{t}=4.1726, \mathrm{p}=0.0087$, standard error of difference $=0.316$ ) from both curricula. This is also true when we compare 
only lessons from curricula $\mathrm{A}(\mathrm{df}=2, \mathrm{t}=3.5905$, $\mathrm{p}$-value $=0.0696$, standard error of difference $=0.367)$.

Table 5.5: Number of words per conversational turn - Paired Lessons - Curriculum A

\begin{tabular}{|rrrr|}
\hline Lesson & \multicolumn{3}{c|}{ Experimental Condition } \\
& All Sighted & With the system & Without the system \\
\hline 1 & 11.39 & 10.29 & 10.89 \\
2 & 11.84 & 9.54 & 11.35 \\
3 & 9.21 & 9.73 & 11.27 \\
\hline Mean & 10.81 & 9.85 & 11.17 \\
Std Dv & 1.41 & 0.39 & 0.25 \\
\hline
\end{tabular}

Such difference was not found when we analyzed only curriculum B lessons $(\mathrm{df}=2, \mathrm{t}=$ 2.1860, p-value $=0.1604$, standard error of difference $=0.604$ ).

Table 5.6: Number of words per conversational turn - Paired Lessons - Curriculum B

\begin{tabular}{|crrr|}
\hline Lesson & \multicolumn{3}{c|}{ Experimental Condition } \\
& All Sighted & With the system & Without the system \\
\hline 1 & 13.25 & 13.35 & 15.69 \\
2 & 11.47 & 12.64 & 14.01 \\
3 & 14.38 & 14.97 & 15.22 \\
\hline Mean & 13.03 & 13.65 & 14.97 \\
Std Dv & 1.47 & 1.19 & 0.87 \\
\hline
\end{tabular}

Table D.1 on page 302 has a complete listing of the mean number of words uttered by the instruction per conversational turn per trial.

\section{- Conclusions:}

The use of the system led to more economical conversational turns when comparing both curricula (Paired Students $t$ test). This is also true when we investigate only curriculum A lessons. For curricula B lessons, albeit not significant, a clear trend for the more economical conversational turns is identified also when the system was used.

\section{Duration of instructor's conversational turns - DIT}

- How it was measured - Each conversational turn was transcribed along with the time it started and the time it ended. Times were obtained from the tapes. Similarly to 
what we did in the previous analysis, we will first compare DIT across the experimental conditions to have an overall understanding of the eventual changes - the overall comparison. We also performed the paired analysis using paired Student's $t$ test at $90 \%$ confidence interval considering both curricula together and each one individually.

- Overall comparison - Figure 5.4 and table 5.4 show results obtained from the overall analysis. The data suggests no overall trend.

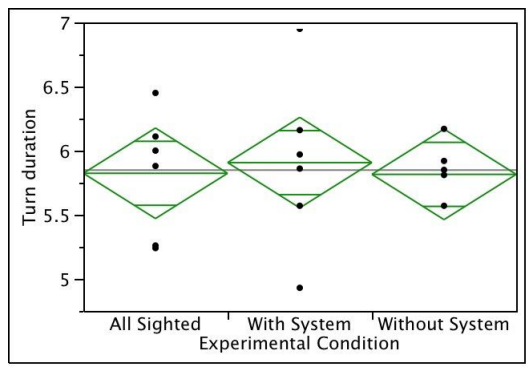

Figure 5.5: Turn duration (Instructor) - in seconds

Table 5.7: Turn duration (Instructor) - Overall comparison

\begin{tabular}{|lrrrrr|}
\hline Level & $n$ & Mean & Std Error & Lower 90\% & Upper 90\% \\
\hline All Sighted & 6 & 5.823 & 0.201 & 5.470 & 6.176 \\
With System & 6 & 5.907 & 0.201 & 5.554 & 6.260 \\
Without System & 6 & 5.815 & 0.201 & 5.462 & 6.168 \\
\hline
\end{tabular}

- Paired lessons analysis - No significant difference was found when we compared both curricula together $(\mathrm{df}=5, \mathrm{t}=0.1377$, $\mathrm{p}$-value $=0.8958$, standard error of the difference $=0.303$ ) nor individually (Cur $\mathrm{A}-\mathrm{df}=2, \mathrm{t}=0.2118, \mathrm{p}=0.8519$, standard error of difference $=0.567$, table 5.8, Cur $\mathrm{B}-\mathrm{df}=2, \mathrm{t}=0.7070, \mathrm{p}=0.7933$, standard error of difference $=0.429$, table 5.9 ).

Table D.2 on page 303 has a complete listing of the average duration of the instructors' conversational turns

- Conclusions: The results were not statistically conclusive and no trend was identified. One possible explanation is that pointing acts in the "with the system" trials that triggered longer turns were so few that its effect on turn duration got diluted. 
Table 5.8: Average of turn duration - Paired Lessons - Curriculum A

\begin{tabular}{|rrrr|}
\hline Lesson & \multicolumn{3}{c|}{ Experimental Condition } \\
& All Sighted & With the system & Without the system \\
\hline 1 & 6.11 & 6.16 & 5.57 \\
2 & 6.45 & 5.86 & 5.57 \\
3 & 5.24 & 4.93 & 6.17 \\
\hline Mean & 5.93 & 5.65 & 5.77 \\
Std Dv & 0.62 & 0.64 & 0.34 \\
\hline
\end{tabular}

Table 5.9: Average of turn duration - Paired Lessons - Curriculum B

\begin{tabular}{|rrrr|}
\hline Lesson & \multicolumn{3}{c|}{ Experimental Condition } \\
& All Sighted & With system & Without the system \\
\hline 1 & 6.00 & 5.97 & 5.92 \\
2 & 5.26 & 5.57 & 5.85 \\
3 & 5.88 & 6.95 & 5.81 \\
\hline Mean & 5.71 & 6.16 & 5.86 \\
Std Dv & 0.40 & 0.71 & 0.06 \\
\hline
\end{tabular}

\section{Hand positioning events - HPE}

- How it was measured - We counted the times the instructor stopped the lecture, walked up to the SBVI and physically repositioned her hand to maintain her situated in the instruction. Similarly to the previous measures, we also did both the overall and paired comparisons.

- Overall comparison - Figure 5.6 and table 5.10 show results obtained from the overall analysis. One can observe that inclusive classrooms where the system is used tend to be interrupted fewer times.

Table 5.10: Hand positioning (Instructor) - Overall comparison

\begin{tabular}{|lrrrrr|}
\hline Level & $n$ & Mean & Std Error & Lower 90\% & Upper 90\% \\
\hline With System & 6 & 0.305 & 0.484 & -0.572 & 1.182 \\
Without System & 6 & 1.962 & 0.484 & 1.085 & 2.839 \\
\hline
\end{tabular}




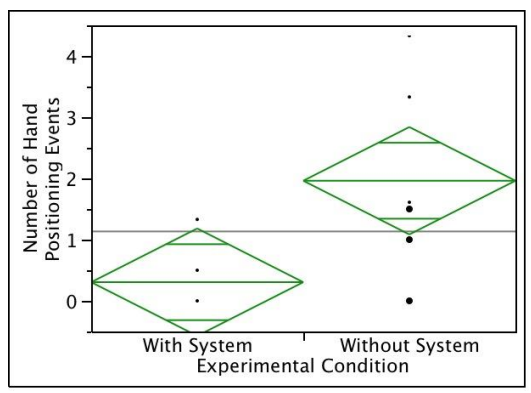

Figure 5.6: Hand positioning events

- Paired lessons analysis - When we compared both curricula together, we concluded that the use of the system did lead to fewer lecture interruptions to attend the blind $(\mathrm{df}=5, \mathrm{t}=2.2516, \mathrm{p}$-value $=0.0741$, standard error of difference $=0.736)$. This is also true when we compared only curriculum A lessons $(\mathrm{df}=2, \mathrm{t}=3.8902$, $\mathrm{p}$-value $=0.0602$, standard error of difference $=0.794$, table 5.11). However, no significant difference was found for curriculum B lessons $(\mathrm{df}=2, \mathrm{t}=1.5215$, $\mathrm{p}$-value $=0.2675$, standard error of difference $=$ 0.147 , table 5.12.

Table 5.11: Hand Positioning Events - Paired Lessons - Curriculum A

\begin{tabular}{|rrr|}
\hline Lesson & \multicolumn{2}{c|}{ Experimental Condition } \\
& With the system & Without the system \\
\hline 1 & 0.00 & 1.61 \\
2 & 0.00 & 4.33 \\
3 & 0.00 & 3.33 \\
\hline Mean & 0.00 & 3.09 \\
Std Dv & 0.00 & 1.38 \\
\hline
\end{tabular}

Table D.3 on page 304 has a complete listing of the hand positioning events.

\section{- Conclusions:}

Important differences were found between instructors/curricula. Curriculum A instructor never touches the student during "with the system" lectures. Whereas in his "without the system" trials, he stops 6, 4 and 3 times in each of his lesson 2 lectures. It seems that Curriculum B instructor did not change her behavior with regard to hand positioning due to the system. However, she was very cautious when taking the student's hand. She would say: "I don't want to scare you to death $<<$ laughing $>>$ ", 
Table 5.12: Hand Positioning Events - Paired Lessons - Curriculum B

\begin{tabular}{|rrr|}
\hline Lesson & \multicolumn{2}{c|}{ Experimental Condition } \\
& With the system & Without the system \\
\hline 1 & 0.00 & 0.00 \\
2 & 1.33 & 1.50 \\
3 & 0.50 & 1.00 \\
\hline Mean & 0.61 & 0.83 \\
Std Dv & 0.67 & 0.76 \\
\hline
\end{tabular}

when approaching the student. At this point we still do not have enough data to draw any further conclusion.

\section{Percentage of conversational turns aimed at the lesson's objectives - TLO}

- How it was measured - We classified each conversational turn (instructor and students) as belonging to either one of the three discourse levels: Object level, Meta level and Para level. In object level, instructor and students are debating issues related to the lessons objectives. Whereas in meta level, the subject is the discourse itself. Turns related with discourse repair were also coded as meta level. Para level utterances relate to the individual experience. Words of encouragement like: "There you go", "take your time", etc were coded as as belonging to the para level thread. We report the percentage of object level turn.

- Overall Comparison - Figure 5.13 and table 5.13 show results obtained from the overall analysis. Here we also see that inclusive classrooms where the system is used tend to be more focused on the lesson's objectives then those where the system is not used.

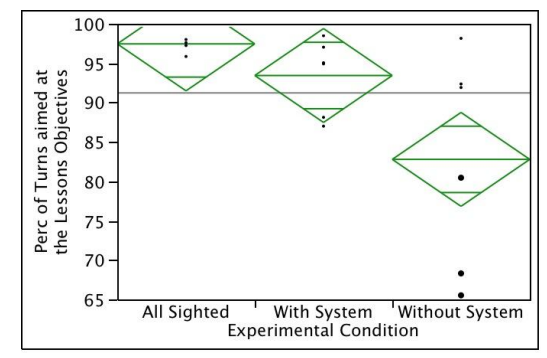

Figure 5.7: Focus on lesson's objectives - Overall comparison 
Table 5.13: Focus on lesson's objectives - Overall comparison

\begin{tabular}{|lrrrrr|}
\hline Level & $n$ & Mean & Std Error & Lower 90\% & Upper 90\% \\
\hline All Sighted & 6 & 97.447 & 3.401 & 91.485 & 103.410 \\
With System & 6 & 93.433 & 3.401 & 87.472 & 99.390 \\
Without System & 6 & 82.782 & 3.401 & 76.820 & 88.740 \\
\hline
\end{tabular}

- Paired lessons analysis - We found no significant difference when comparing all lessons of both curricula together $(\mathrm{df}=5, \mathrm{t}=0.9616, \mathrm{p}$-value $=0.3804$, standard error of difference $=5.707)$. However, the system made instructors and students from curriculum A more focused on the lessons objectives $(\mathrm{df}=2, \mathrm{t}=1.8063$, $\mathrm{p}$-value $=0.0427$, standard error difference $=8.383$, table 5.14). Conversely, instructor and students in curriculum $B$ were less focused when they were using the system $(\mathrm{df}=2, \mathrm{t}=6.5023, \mathrm{p}$-value $=0.0228$, standard error of difference $=0.635$, table 5.15 .

Table 5.14: Discourse Level - Paired Lessons - Curriculum A

\begin{tabular}{|rrrr|}
\hline Lesson & \multicolumn{3}{c|}{ Experimental Condition } \\
& All Sighted & With the system & Without the system \\
\hline 1 & 95.85 & 98.49 & 88.75 \\
2 & 98.00 & 97.03 & 65.52 \\
3 & 97.60 & 95.05 & 90.98 \\
\hline Mean & 97.15 & 96.86 & 81.75 \\
Std Dv & 6.9 & 2.51 & 1.47 \\
\hline
\end{tabular}

Table 5.15: Discourse Level - Paired Lessons - Curriculum B

\begin{tabular}{|rrrr|}
\hline Lesson & \multicolumn{3}{c|}{ Experimental Condition } \\
& All Sighted & With the system & Without the system \\
\hline 1 & 97.24 & 88.12 & 91.91 \\
2 & 97.99 & 87.00 & 92.36 \\
3 & 98.00 & 94.91 & 98.15 \\
\hline Mean & 97.74 & 90.01 & 94.14 \\
Std Dev & 2.61 & 3.97 & 1.17 \\
\hline
\end{tabular}

Table D.4 on page 305 has a complete listing of the lessons and the percentage of object level turns. 
- Conclusions: Important differences between instructors/curricula were observed. Instructor and students from curriculum A where more focused on the lesson's objectives when the system was used. On the other hand, instructor and students from curriculum B where less focused on the lesson's objectives when the system was used. When comparing all lessons together, we found a trend towards more focus on lecture objectives when the system was used because the effect from curriculum A was stronger than that of curriculum B. Maybe the fact that Curriculum B had less complex figures made landmark navigation was easier during the "without the system trials". Another possible explanation is that in three of the Curriculum B trials (held in the same day), $\mathbf{S}$ was wearing colorful clothes which confounded our color based tracking sub-system.

\section{Deictic Expressions - DE}

- How it was measured - Each conversational turn was automatically parsed for words like: here, there, that. A second and manual parse assigned those turns as either containing or not a deictic expression. The number showed that represents the percentage of turns having deictic expressions (number of turns having deictic expressions/total turns).

- Overall Comparison - Figure 5.8 and table 5.16 show the results obtained from the overall analysis. This superficial analysis strongly suggests that instructors used more deictic expressions when using the system than when not using it. The paired lessons analysis confirms the finding.

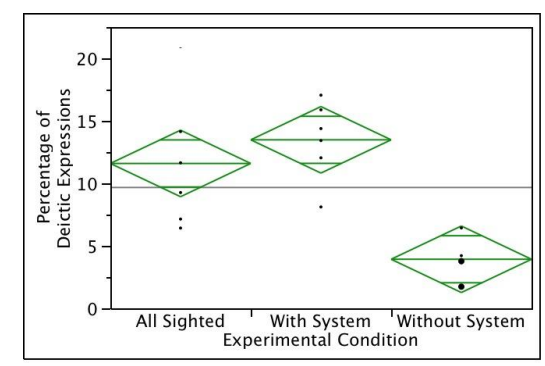

Figure 5.8: Deictic Expressions - Overall comparison

- Paired lessons analysis - When comparing all lessons from both curricula, we found that the instructors used more deictic expressions in their "with the system" trials $(\mathrm{df}=5, \mathrm{t}=8.1477$, $\mathrm{p}$-value $=0.0005$, standard error of difference $=1.172)$. This is true also when we are comparing lessons belonging to the same curriculum (Curriculum A $d f=2, t=14.335, p$-value $=0.00049$, standard error of difference $=0.678$, table 5.17 ; 
Table 5.16: Deictic Expressions - Overall Comparison

\begin{tabular}{|lccrrr|}
\hline Level & $n$ & Mean & Std Error & Lower 90\% & Upper 90\% \\
\hline All Sighted & 6 & 11.590 & 1.518 & 8.929 & 14.251 \\
With System & 6 & 13.480 & 1.518 & 10.819 & 16.141 \\
Without System & 6 & 3.925 & 1.518 & 1.264 & 6.586 \\
\hline
\end{tabular}

Curriculum $\mathrm{B} d \mathrm{~d}=2, \mathrm{t}=3.7152$, $\mathrm{p}$-value $=0.0654$, standard error of difference $=2.527$, table 5.18).

Table 5.17: Percentage of Deictic Turns - Paired Lessons - Curriculum A

\begin{tabular}{|rrrr|}
\hline Lesson & \multicolumn{3}{c|}{ Experimental Condition } \\
& All Sighted & With the system & Without the system \\
\hline 1 & 7.14 & 12.04 & 3.66 \\
2 & 14.14 & 17.05 & 6.43 \\
3 & 20.94 & 14.38 & 4.21 \\
\hline Averages & 14.07 & 14.49 & 4.77 \\
\hline
\end{tabular}

Table 5.18: Deictic Turns - Paired Lessons - Curriculum B

\begin{tabular}{|rrrr|}
\hline Lesson & \multicolumn{3}{c|}{ Experimental Condition } \\
& All Sighted & With the system & Without the system \\
\hline 1 & 6.42 & 13.42 & 1.73 \\
2 & 11.64 & 15.88 & 3.75 \\
3 & 9.26 & 8.11 & 3.77 \\
\hline Averages & 9.11 & 12.47 & 3.08 \\
\hline
\end{tabular}

Table $\mathrm{D} .5$ on page 306 has a complete listing all trials and their respective percentage of instructor's deictic expressions.

\section{- Conclusions:}

Instructors used significantly more deictic expressions when using the system than when not using it In "without the system" trials. Furthermore instructors used deictic expressions only when holding the student's hand. This shows that the introduction of the system had an impact on the instructor's utterance formation, demonstrating that the instructors were confident that SBVI would understand their behavior. 


\section{Summary of the Lecture Fluency Analysis}

Table 5.19 shows a summary of the lecture fluency measures (Paired lessons - "with" against "without the system" comparisons). The $A+B$ column denotes the tests where pairs of both curricula were taken into consideration when running our paired Student's $t$ test. Consequently, columns A and B correspond to the intra-curricular comparisons. This table is presented as a separate section for the reader's connivence. A complete and final discussion on the instruction study is found on 149 . Before that, however, we will still debate on the instructors' and students' experience and on learning outcome, the following sections.

Table 5.19: Summary of the comparisons on Lecture Fluidity Measures

\begin{tabular}{|c|c|c|c|}
\hline Measure & \multicolumn{3}{|c|}{ Currriculum } \\
\hline & $\overline{A+B}$ & $\overline{\mathrm{A}}$ & $\overline{\mathrm{B}}$ \\
\hline Words per Turn & $\begin{array}{l}\text { Less words per } \\
\text { turn when the } \\
\text { system was used } \\
\text { Trend }\end{array}$ & $\begin{array}{l}\text { Less words per } \\
\text { turn when the } \\
\text { system was used } \\
\text { Sign Diff }\end{array}$ & Inconclusive \\
\hline $\begin{array}{l}\text { Duration of In- } \\
\text { structors turn }\end{array}$ & \multicolumn{3}{|c|}{ No difference found } \\
\hline $\begin{array}{l}\text { Hand Positioning } \\
\text { Events }\end{array}$ & $\begin{array}{l}\text { Less hand po- } \\
\text { sitioning events } \\
\text { when the system } \\
\text { was used } \\
\text { Sign Diff }\end{array}$ & $\begin{array}{l}\text { Less hand po- } \\
\text { sitioning events } \\
\text { when the system } \\
\text { was used } \\
\text { Sign Diff }\end{array}$ & Inconclusive \\
\hline $\begin{array}{l}\text { Focus on lessons } \\
\text { objectives }\end{array}$ & $\begin{array}{l}\text { More focus when } \\
\text { the system was } \\
\text { used } \\
\text { Trend }\end{array}$ & $\begin{array}{l}\text { More focus when } \\
\text { the system was } \\
\text { used } \\
\text { Sign Diff }\end{array}$ & $\begin{array}{l}\text { Less focus when } \\
\text { the system was } \\
\text { used } \\
\text { Sign Diff }\end{array}$ \\
\hline $\begin{array}{l}\text { Deictic } \\
\text { sions }\end{array}$ & $\begin{array}{l}\text { More deictic ex- } \\
\text { pressions when } \\
\text { the system was } \\
\text { used } \\
\text { Sign Diff }\end{array}$ & $\begin{array}{l}\text { More deictic ex- } \\
\text { pressions when } \\
\text { the system was } \\
\text { used } \\
\quad \text { Sign Diff }\end{array}$ & $\begin{array}{l}\text { More deictic ex- } \\
\text { pressions when } \\
\text { the system was } \\
\text { used } \\
\text { Sign Diff }\end{array}$ \\
\hline
\end{tabular}




\subsubsection{Instructors' and Student's Experience}

Post-questionnaires were conducted verbally after the trials. These took the form of a set of statements to which the participants responded on an agreement Likert scale (1-strongly disagree to 5 -strongly agree, 3 being no opinion). All participants (instructors and students) were urged to comment on their answers. Table 5.20 shows where post-questionnaires can be found.

Table 5.20: Post Questionnaires

\begin{tabular}{|c|c|c|}
\hline Participant & Experimental Condition & Page No \\
\hline Instructor & With the system & 256 \\
SBVI & $\begin{array}{c}\text { Without the system } \\
\text { Sighted Student }\end{array}$ & $\begin{array}{c}\text { With the system } \\
\text { Without the system }\end{array}$ \\
& $\overline{286}$ \\
\hline
\end{tabular}

We shall now discuss how each group of participants perceived the experience.

\section{Instructors}

Before we begin, we must recall that instructor $\mathbf{A}$ taught curriculum A, while $\mathbf{S}$ taught curriculum B. They both agreed that the instructional material used in the courses was properly formatted, but they said would make changes. "I would make many more graphs. It would take more preparation than normal. But it would make you a better instructor overall", A. For A, the SBVI showed less confusion when the system was used, whereas S had no formed opinion. "They would get more frustrated", A; "They seem to adapt", S.

Both instructors agreed that they were able to express themselves more effectively when the system was used. "Because I could continue being the instructor instead of going to a particular student and help him find something. They could be finding while I was talking and keeping up with the instruction", said S. A and S strongly agreed that the instructor's display helped them understanding the student behavior. "It might be distracting in a larger class", said A. They all agreed that the system will be useful in real world teaching.

"I'm excited to see where it goes. Have you applied for patent yet? I have a nephew who works at US patent office. I need to call him and say: 'Watch for this' (Laughs)", said S. When asked if they thought that the SBVI understood the lectures better when they were using the system than when they were not, A strongly agreed, while S: "It really depends on the blind students themselves", $\mathbf{S}$. 
Both strongly agreed that the system helped instructor/student interaction. "I didn't have to think of what I was going to say (verbalize) as much as I did without the system. It helped the conversation flow more like a normal every day conversation", A.

Did the system slow down or speed up the lecture?, we asked. "It sped up because I didn't have to go over the student", A. "It somewhat slowed it. It is probably advantageous that it slowed it down. When teaching the only feedback you have is eye contact. The system made me acutely aware that the student was not paying attention", $\mathbf{S}$.

Both would rather teach inclusive classrooms using the system. They all agreed that those who attended classes where the system was used would have a better chance on the exams. "Because there was less confusion", A. "I think you may see some positive results", S. How did the system impact the SBVI engagement in class discussion?, we asked. "It promoted it", A. "All blind students were very engaged. No matter what. More than the sighted", S.

\section{SBVI post-questionnaire}

Before we begin presenting the SBVIs' answers and opinions, we must disclose relevant information about the trials. The trials were held in the Learning with Disabilities lab at Wright State University (WSU), Dayton, Ohio. This study was performed during the two weeks before the finals week. Due to class schedule at WSU, $A$ (SBVI) was available for only one day each week. Our study lasted two week, so she had to take all three lessons of each course in one day. $A$ arrived one and a half hours late for her trial with the system. Furthermore, $S$, her instructor for that trial was wearing colorful clothes which made our color based tracking algorithm less effective. $\quad R$ also had all lessons with the system in the same day. When greeted upon his arrival for the trial, he said: "I'm hungry, tired and broke".

For the above reasons we have two distinct groups of opinions. In group 1 (G1), we have: $O, N$ and $G$. $A$ and $R$ form group 2 (G2).

All but $R$ agreed that playing the game and the charade helped them to get ready for class. "This is comparing apples to green beans. The game was important for us to get comfortable with the glove, but the task was completely different. The charade was closer. Attending more (with the system) classes would help", $R$

All but $\mathrm{R}$ agreed the the instruction material was formatted properly. "Labels were far from the point on the figure to where they refer", $R$. However, they all agreed that they did not have problems understanding the labels. G1 members reported that they were comfortable using the system in class, while those from G2 had no opinion. Only $A$ perceived the system as a impediment to keeping up with the lecture. "Mathematics is so demanding. You have to listen and follow along and using the system can be overwhelming", A.

They all felt that instructors paid more attention to them in classes where the system was used. They all agreed that using the system did not stress them. In which circumstances you lost track of the instructor?, we asked. $N, O, A$ reported that due to the system they lost 
track of the instructor fewer times. "There were times I had no idea of what she was talking about", $N$, on her "without the system" trials. $G$ got lost on the when the system was used. "I got lost when he moved his wand around very fast", G, complaining that sometimes the instructor got carried away and did not wait for him. "The instructor drew new figures and pointed at them with the wand and I lost that", said G.

G1 members found it easier to understand mathematics concepts when the system was used, while $R$ preferred the classes where the system was not used. $A$ had no preference. They all agreed that they could clearly understand the graphics used in the mini-courses in both scenarios.

Will the use of the system improve your chances of getting a better grade?, we asked. $N$ and $O$ said "Yes". $R$ said "No", while G, A no formed opinion "Teaching style is more important", G. All five participants agreed that the system will be useful in real classrooms. "The performance of both instructor and student will improve as they use the system", $R$. "But not for math. Math is too demanding", emphasized A.

\section{Sighted Students post-questionnaire}

Twelve sighted students, who took courses with and without the system, were interviewed. Ten said that the system did not disrupt the lecture flow. They all agreed that "with the system" classes were more fluent than those were the system was not used. Eight would rather attend inclusive classrooms where the system is used we four were indifferent. Table 5.21 shows a list of comments the sighted students made. In that table, the column choice displays one of the two values: A when the student said she should rather attend inclusive classrooms with the augmented technology or $\mathbf{I}$ for those who were indifferent.

Finally, none of the sighted students felt intimidated with the cameras and they all believe that the system will be useful in real world teaching. 
Table 5.21: Comments Sighted Students' made when comparing attending inclusive classes with and without the system

\begin{tabular}{|lcc|}
\hline Commentary & Participant & Choice \\
"The system made the lecture flow more easily" & $S$ & A \\
"She (the student who is blind) was able to follow along easier" & $C$ & A \\
"I would rather have it, then not. It made things a lot more & $C J$ & A \\
easier." & & \\
"It helped go a lot smoother. The teacher wasn't constantly & $C J$ & $\mathrm{~A}$ \\
trying to show where the student need to go. She the student & \\
who is blind) was more indenpent, I guess" & & \\
"I thought it helped O and she was able to participate and see & $C H$ & $\mathrm{~A}$ \\
what was going on. She was able to visualize what we were talking & & \\
about (the graphs)" & & \\
"I liked the system. It helped the teacher to keep up with the & $C K$ & $\mathrm{~A}$ \\
lesson and instead of stopping to help the student" & & \\
"The system didn't make any difference (to me)" & $\mathrm{I}$ \\
"It really doesn't matter to me, as long as I'm understanding ..." & $H$ & $\mathrm{I}$ \\
"I think it made the teacher pay more attention to make sure that & $H$ & $\mathrm{I}$ \\
we understood the material." & & \\
\hline
\end{tabular}




\section{Learning Outcome}

As presented on the last section, instructors think that the system improved the quality of the lecture mainly because it raised their awareness of the SBVI behavior enabling them to act upon any signs of confusion to assure understanding. The sighted students also believed that SBVI were more engaged in classroom discussions and showed more signs of understanding when the system was used. Our situated analysis also suggests that when the system was used, the instructor and SBVI were more likely to share growth points and hyperphrases than when it was not. It also demonstrated that the use of the system created more evidences of mutual understanding when compared to the "without the system" condition.

These are all good indicators. However, opinions were divided among the SBVI. The ones who were on a "good day" when they attended classes with the system gave higher marks for the leaning issue. Others like $G$ did not like the fact that the instructor used the wand to make other gestures than pointing. It is also important to acknowledge that because of the novelty of the system and lack of proper instructor training, the system also created new situations of confusion, like the one above. It is also important to note that they all agreed that the system was not a source of stress during lecture, and they perceived that instructors paid more attention to them during the "with the system" trials.

The discussion above lead us to believe that those SVBI who attended "with the system" lectures would get better grades on the exams. However, as pointed before, other factors, maybe even more important than the system, have impact on exam performance. As $G$ properly pointed: "teaching style is more important". Previous knowledge and which curriculum was covered in which experimental conditions are equally important factors. Add to these, the unfortunate fact that a tape containing oral exam of two of our five SBVI was lost, making our already precarious statistical analysis impossible. We, therefore, reach the end of this discussion knowing that there are good exciting signs that system might improve learning, however, a definitive conclusion is beyond the reach of the present study.

\subsection{Overall conclusions from the Instruction Study}

We now present our general conclusions from the instruction study.

1. The introduction of the system:

(a) Creates concrete learning opportunities. As we have shown on the situated analysis section, the system creates opportunities for growth point sharing between instructor and SBVI. Furthermore, SBVI can still extract meaning from the instructor, through hyperphrase, in cases when the instructor's speech and gesture synchrony was less than perfect. 
(b) Creates new opportunities for confusion and frustration for the student. Such situations normally happen when instructors do not wait for the SBVI to resolve the referent or when, inadvertently, instructors use the wand to make gestures other than pointing.

(c) Creates more perceptual cues that can be used for increasing mutual understanding between instructor and SBVI and consequently improving the quality of the lecture. We also demonstrated that instructor and SBVI relied on HDS components (instructor's display and haptic glove) to coordinate collective actions related to teaching/ learning.

(d) Increases lecture fluency in inclusive classrooms. Our lecture fluency analysis clearly shows the improvement.

(e) Demands instructor training. Instructors need to understand system's limitations and the impact of their actions on the SBVI's. In this study, HDS was set to track a wand instructors used to point at relevant portions of the figures. In some occasions, instructors wove the wand while performing gestures other than pointing. The HDS tracked and delivered those gestures, confusing the students. Some times the instructors deviated from the prepared graphical material and wrote on the whiteboard. While this is totally normal in regular classes, the attitude tends to hinder the SBVI understanding because she will not benefit from braille/raised line notes she was given.

(f) Requires careful lecture planning. Curricula and lecture notes (including figures) were prepared by our research team. During debriefing sessions, instructors suggested the use of more figures. In the "all sighted" lectures, instructors drew several figures on the board to convey specific concepts. In the inclusive trials, those concepts were conveyed using specific areas of the the figures given. The "ad-hoc" drawn figures were simpler and showed only the graphical aspect relevant to the concept being conveyed. This corroborates $A$ 's opinion: "I would have prepared much more graphs". Thus, future curricula should have more and simpler graphs. Another important issue is related to calculations performed during lecture. Obviously, the SBVI could not follow the instructor's writing on the board. SBVI's class notes should include all the calculations the instructor will perform during lecture. Furthermore, when those calculations make references to the elements of a figure, that figure must remain accessible.

2. The SBVI were able to follow classroom instruction using the system. The SBVI benefited from the multimodal cues available to them. The instructor's speech, the raised line document and directional signals coming from the haptic glove helped the students navigating to where the instructor was pointing must faster than we anticipated. We posit, agreeing with Oviatt [208], that this multimodal sensory information helped resolving ambiguity, improving performance.

3. Both Instructors, all SBVI and all sighted students believe the technology will be useful 
in real classrooms. All study participants showed enthusiasm about the $H D S$ - Even the SBVI who were on a "bad day" when they tried the system. They found the technology non-disruptive, and that the "with the system" lessons flowed naturally.

4. Instructors prefer to lecture inclusive classrooms with the system. The use of the system changed the way instructors formulated their sentences. The use of deictic expressions (present only in the "with the system" trials) lowered the utterance formation/understanding costs for both instructors and students. This freed cognitive resources for both sides of the teaching/learning equation.

5. Instructors indicated that the awareness of where the SBVI were reading was valuable in-an-of-itself. The information helped them to find the right pace of the lecture, ensuring that all students were following the lecture.

6. One SBVI did not like the fact that the system gave the instructor "extra powers" like knowing all the time whether they were paying attention or not. Interestingly, instructors asked for a similar tool for the sighted students. They wanted to have extra and definitive evidence of who, among the sighted, is paying attention and who is not.

7. Sighted students prefer to attend inclusive classrooms where the system is used. We wondered if the $H D S$ would also benefit the sighted students, if it had created the so-called curb-cut effect. One obvious benefit was the less interruptions during the lecture (less hand positioning events) when the system was used. A more subtle one would come from the fact that instructors pointed at the relevant areas of the figures and hold their posture until the SBVI could find them. This longer display of what the instructor considered important for the understanding of the concept being conveyed could also benefit the sighted. Unfortunately, we could not find any evidence of that.

\subsection{New Opportunities}

SBVI should be able to do "cognitive offloading" by taking notes that could be revisited later. The need for tutor could be lessened if we had a second learning tool especially designed for studying. Such tool could make use of audio and video captured from the lectures as well as the instructor's pointing acts. All students (blind and sighted) could benefit from such tool which could promote the creation of an inclusive learning community. As discussed on chapter 4 (Games and Accessibility), designing the right interface should further social inclusion. This is one particularly rewarding research area for the HumanComputer Interaction community.

We also observed how hard it is for a SBVI to perform calculations (figure 5.9). We advocate a tool similar to that described by Bolt [23], where the user points at a wall and the system 
recognizes both her pointing and deictic expressions (speech input). With such tool, the SBVI could offload cognitive work on the environment, such like the sighted do [296]. When solving complex mathematics or science problems, the SBVI could "store" partial results in space, recollecting them as they were needed.

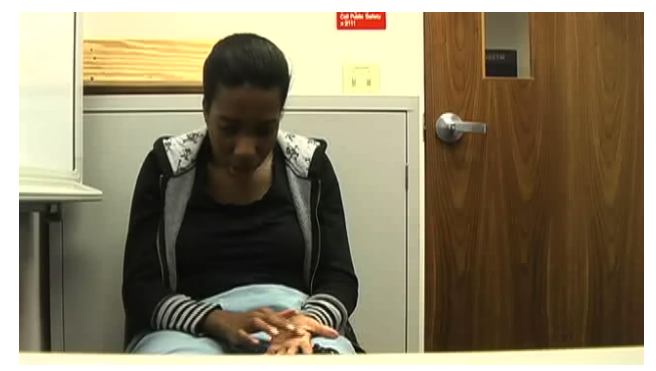

Figure 5.9: Need to do cognitive offloading - "O" is writing on her palm

\subsection{Summary of the Work Completed}

We proposed, developed, tested and evolved a system conceived to help students who are blind or visually impaired understand the instructor's pointing gestures. Our main scientific motivation was to investigate the impacts that such change would bring to the conveyance of graphical mathematics concepts to those who cannot see.

We started creating a robust and comfortable haptic glove capable of producing strong and clear directional signals. In our first usability studies, we show that: 1) The glove is able to convey sense of direction; 2) It does not interfere in fingertip reading; 3) A person can navigate with the help of this system while listening to a story and 4) It is possible to fuse the information received from both senses.

Follow up studies show that the assistive technology use must become embodied and automatic before it can support dynamic fluent instructional discourse. We investigated a strategy where blind users develop skill in a fun and challenging way through a skill training game. Skills were transferable to the complex multimodal situated discourse condition.

Subsequently, our system was employed in mathematics classes attended both by SBVI and regular students. For instructors, the technology allowed them to: 1) Adjust the pace of the lecture to ensure that all students were following them; 2) Better understand the students signs of confusion and act upon them to ensure their understanding; 3) Act more naturally as they did not have to think of how to verbalize the information displayed on the graphs. Overall, instructors agree that the use of the technology improved the quality of instruction. The SBVI were able to comprehend, more quickly and effectivelly, the instruction when they 
were using the system. For the sighted students, the system: 1) Improved lecture fluidity; 2) Made the SBVI more participative in classroom discussions, and 3) Did not make the instructors pay less attention to them.

Finally, we presented future research opportunities that could help solving some of the problems and difficulties observed in our studies. 


\section{Bibliography}

[1] about.com. What is the circumference of the earth?

[2] M. Akamatsu and S. Sato. A multi-modal mouse with tactile and force feedback. International Journal of Human-Computer Studies, 40(3):443-453, 1994.

[3] A. Anderson, E. Bard, C. Sotilo, G. Doherty-Sneddon, and A. Newlands. The effects of face-to-face communication on the intelligibility of speech. Perception $\mathscr{E}$ psychophysics, 59:580-592, 1997.

[4] D. Archambault and D. Burger. TIM (Tactile Interactive Multimedia): Development and adaptation of computer games for young blind children. In Proc. ERCIM WG UI4ALL 83 i3 Sping Days 2000 Joint workshop, Interactive Learning Environments for Children, 2000.

[5] D. Archambault and D. Olivier. How to make games for visually impaired children. In Proceedings of the 2005 ACM SIGCHI International Conference on Advances in computer entertainment technology, pages 450-453. ACM New York, NY, USA, 2005.

[6] D. Archambault, R. Ossmann, T. Gaudy, and K. Miesenberger. Computer games and visually impaired people. Upgrade, 8(2):43-53, 2007.

[7] M. Argyle and J. Graham. The Central Europe Experiment-looking at persons and looking at things. Journal of Environmental Psychology and Nonverbal Behaviour, 1(1977):6-16, 1977.

[8] M. Argyle, M. Lalljee, and M. Cook. The effects of visibility on interaction in a dyad. Human Relations, 21(1):3, 1968.

[9] J. Atkinson, M. Francis, and F. Campbell. The dependence of the visual numerosity limit on orientation, colour, and grouping of the stimulus. Perception, 5(3):335-342, 1976.

[10] M. Atkinson, S. Gucukoglu, C. Machin, and A. Lawrence. Making the Mainstream Accessible: What's in a Game? Lecture Notes in Computer Science, 4061:380, 2006.

[11] P. Bach-y Rita. Brain Mechanisms in Sensory Substitution. Academic Press Inc, 1972. 
[12] P. BACH-Y-RITA. Tactile Sensory Substitution Studies. Annals of the New York Academy of Sciences, 1013(1):83-91, 2004.

[13] P. Bach-y Rita, C. Collins, F. Saunders, B. White, and L. Scadden. Vision substitution by tactile image projection. Nature, 221(5184):963-964, 1969.

[14] R. Baear, R. Flexer, and R. K. McMahan. Transition models and promising practices. In Transition Planning for Secundary Students with Disabilities, pages 53-82, 2005.

[15] E. Baker, M. Wang, and H. Walberg. The effects of inclusion on learning. Educational Leadership, 52(4):32-35, 1994.

[16] J. Bardin. Academic Engagement of Students with Visual Impairments in General Education Language Arts Classrooms. PhD thesis, The Florida State University, 2006.

[17] G. Beattie and H. Shovelton. Mapping the range of information contained in the iconic hand gestures that accompany spontaneous speech. Journal of Language and Social Psychology, 1999.

[18] E. Berlá. Haptic perception of tangible graphic displays. Tactual perception: a sourcebook, pages 364-386, 1982.

[19] K. Bierre, M. Hinn, T. Martin, M. McIntosh, T. Snider, K. Stone, and T. Westin. Accessibility in Games: Motivations and Approaches. http://www.igda.org/ accessibility/IGDA_Accessibility_WhitePaper.pdf, Last checked: 01/26/2010, 2004.

[20] W. Blokzijl and B. Andeweg. The effects of text slide format and presentational quality on learning in college lectures. Professional Communication Conference, 2005. IPCC 2005. Proceedings. International, pages 288-299, July 2005.

[21] S. Bly. A use of drawing surfaces in different collaborative settings. In Proceedings of the 1988 ACM conference on Computer-supported cooperative work, pages 250-256. ACM New York, NY, USA, 1988.

[22] S. Bolanowski Jr, G. Gescheider, R. Verrillo, and C. Checkosky. Four channels mediate the mechanical aspects of touch. The Journal of Immunology, 84(5):1680-1694, 1988.

[23] R. Bolt. "Put-that-there": Voice and gesture at the graphics interface. Proceedings of the 7th annual conference on Computer graphics and interactive techniques, pages 262-270, 1980.

[24] A. Bongers. Palpable pixels, a method for the development of virtual textures. Touch, blindness and neuroscience. UNED Ediciones, Madrid, 2002. 
[25] B. Bongers and G. Van Der Veer. Tactual Articulatory Feedback and Gestural Input. http://citeseerx.ist.psu.edu/viewdoc/download?doi=10.1.1.101. 2126\&rep=rep1\&type=pdf, Last checked: $01 / 26 / 2010$.

[26] J.-Y. Bouguet. Pyramidal implementation of the lucas kanade feature tracker. Technical report, Intel Corporation, 2000.

[27] S. Brewster and L. Brown. Tactons: structured tactile messages for non-visual information display. In Proceedings of the fifth conference on Australasian user interfaceVolume 28, pages 15-23. Australian Computer Society, Inc. Darlinghurst, Australia, Australia, 2004.

[28] S. Brewster and A. King. An Investigation into the Use of Tactons to Present Progress Information. LECTURE NOTES IN COMPUTER SCIENCE, 3585:6, 2005.

[29] W. Brodsky. The effects of music tempo on simulated driving performance and vehicular control. Transportation Research Part F: Psychology and Behaviour, 4(4):219-241, 2001.

[30] J. Brophy and T. Good. Teacher behavior and student achievement. Handbook of research on teaching, 3:328-375, 1986.

[31] L. Brown, S. Brewster, and H. Purchase. A first investigation into the effectiveness of tactons. Eurohaptics Conference, 2005 and Symposium on Haptic Interfaces for Virtual Environment and Teleoperator Systems, 2005. World Haptics 2005. First Joint, pages 167-176, March 2005.

[32] L. Brown, S. Brewster, and H. Purchase. Tactile crescendos and sforzandos: applying musical techniques to tactile icon design. In Conference on Human Factors in Computing Systems, pages 610-615. ACM New York, NY, USA, 2006.

[33] L. Brown and T. Kaaresoja. Feel who's talking: using tactons for mobile phone alerts. In Conference on Human Factors in Computing Systems, pages 604-609. ACM New York, NY, USA, 2006.

[34] A. Buaud, H. Svensson, D. Archambault, and D. Burger. Multimedia games for visually impaired children. Lecture notes in computer science, pages 173-180, 2002.

[35] M. Burke, R. Gilson, and R. Jagacinski. Multi-modal information processing for visual workload relief. Ergonomics, 23(10):961-975, 1980.

[36] A. Cancelli. Type of instruction and the relationship of classroom behavior to achievement among learning disabled children. Journal of Classroom Interaction, 28(1):13-21, 1993. 
[37] A. Carlin, H. Hoffman, and S. Weghorst. Virtual reality and tactile augmentation in the treatment of spider phobia: a case report. Behaviour Research and Therapy, 35(2):153-158, 1997.

[38] J. Cassell. A Framework for Gesture Generation and Interpretation. Computer Vision for Human-Machine Interaction, pages 191-215, 1998.

[39] A. Chapanis, R. Ochsman, R. Parrish, and G. Weeks. Studies in interactive communication. I- The effects of four communication modes on the behavior of teams during cooperative problem-solving. Human Factors, 14:487-509, 1972.

[40] A. Chapanis, R. Parrish, R. Ochsman, and G. Weeks. Studies in interactive communication. II- The effects of four communication modes on the linguistic performance of teams during cooperative problem solving. Human Factors, 19:101-126, 1977.

[41] J. Chen. Flow in games (and everything else). Communications of the ACM, 50(4):3134, 2007.

[42] R. Cholewiak and J. Craig. Vibrotactile pattern recognition and discrimination at several body sites. Perception \&f psychophysics, 35(6):503-514, 1984.

[43] N. Chomsky. The formal nature of language. Biological Foundations of Language, pages 397-442, 1968.

[44] R. J. Christman. Sensory Experience. Harper and Row Publishers, 1979.

[45] E. Churchill, J. Trevor, S. Bly, L. Nelson, and D. Cubranic. Anchored conversations. In Proceedings of CSCW 1996, ACM Conference on Computer-Supported Cooperative Work, 2000.

[46] H. Clark and H. Brownell. Judging up and down. Journal of Experimental Psychology: Human Perception and Performance, 1(4):339-352, 1975.

[47] H. Clark, C. Marshall, and D. Waltz. Theoretical issues in natural language processing. In Proceedings of a conference at the Massachusetts Institute of Technology. In $R$. Schank \&3 B. Nash-Webber (Eds), Cambridge: Harcourt Brace Jovanovitch, 1975.

[48] H. Clark and E. Schaefer. Collaborating on contributions to conversations. Language and Cognitive Processes, 2(1):19-41, 1987.

[49] H. Clark and E. Schaefer. Contributing to discourse. Cognitive science, 13(2):259-294, 1989.

[50] H. Clark and D. Wilkes-Gibbs. Referring as a collaborative process. Intentions in communication, pages 463-493, 1990.

[51] H. H. Clark. Arenas of Language Use. Center for the Study of Language and Inf, 1992. 
[52] H. H. Clark. Using Language. Cambridge University Press, Cambridge, England, 1996.

[53] H. H. Clark and S. Brennan. Grounding in communication. Perspectives on socially shared cognition, 13:127-149, 1991.

[54] M. Clark, H. H. Krych. Speaking while monitoring addressees for understanding. Journal of Memory and Language, 50:62-85, 2003.

[55] P. Cohen. The Pragmatics of Referring and the modality of Communication. Computational Linguistics, 10(2):97, 1984.

[56] C. Collins and F. Saunders. Pictorial display by direct electrical stimulation of the skin. J. Biomed. Syst, 1:3-16, 1970.

[57] J. Craig. Difference threshold for intensity of tactile stimuli. Perception \&3 Psychophysics, 11(2):150-152, 1972.

[58] E. Crowder. Gestures at Work in Sense-Making Science Talk. The Journal of the Learning Sciences, 5(3):173-208, 1996.

[59] M. Csikszentmihalyi. Play and Intrinsic Rewards. Journal of Humanistic Psychology, 15(3):41-63, 1975.

[60] M. Csikszentmihalyi. Flow: the psychology of optimal experience. New York: Harper \& Row, 1990.

[61] R. Daft and R. Lengel. Information Richness. A new approach to managerial behavior and organization design, 1983.

[62] A. D'Angiulli, J. Kennedy, and M. Helle. Blind children recognizing tactile pictures respond like sighted children given guidance in exploration. Scandinavian Journal of Psychology, 39(3):187-190, 1998.

[63] A. de Jorio. Gesture in Naples and Gesture in Classical Antiquity: A Translation of LA Mimica Degli Antichi Investigata Nel Gestire Napoletano, Gestural Expression of the Ancients in the Light of Neapolitan Gesturing. Indiana University Press, 2000.

[64] T. Dick and K. Evelyn. Issues and aids for teaching mathematics to the blind. Mathematics Teacher, 90, 1997.

[65] F. dimension Technologies. http://www.5dt.com/hardware.html\#glove, Last checked: $2 / 27 / 2009$.

[66] J. Dionisio. Virtual hell: A trip through the flames. IEEE Computer Graphics and Applications, 17(3):11-14, 1997. 
[67] L. Dipietro, A. Sabatini, and P. Dario. A Survey of Glove-Based Systems and Their Applications. IEEE Transactions on Systems, Man, and Cybernetics, Part C: Applications and Reviews, 38(4):461-482, 2008.

[68] G. Doherty-Sneddon, A. Anderson, C. O'Malley, S. Langton, S. Garrod, and V. Bruce. Face-to-face and video-mediated communication: A comparison of dialogue structure and task performance. JOURNAL OF EXPERIMENTAL PSYCHOLOGY APPLIED, 3:105-125, 1997.

[69] P. Dourish. Where the Action is: The Foundations of Embodied Interaction. Mit Pr, 2001.

[70] N. Ducheneaut and V. Bellotti. Ceci n'est pas un Objet? Talking About Objects in E-mail. Human-Computer Interaction, 18(1):85-110, 2003.

[71] D. Efron. Gesture, Race and Culture. Mouton, 1972.

[72] M. Endsley. Toward a theory of situation awareness in dynamic systems. Human Factors: The Journal of the Human Factors and Ergonomics Society, 37(1):32-64, 1995.

[73] Euclid. The Elements. The Elements, c. 300 BC.

[74] F. Ferrara, R. Nemirovsky, and C. TERC. Connecting talk, gesture, and eye motion for the microanalysis of mathematics learning. International Group for the Psychology of $M$ athematics Education, page 138, 2005.

[75] T. Finholt, L. Sproull, and S. Kiesler. Communication and performance in ad hoc task groups, Intellectual teamwork: social and technological foundations of cooperative work, 1990.

[76] A. Fisk and A. Schneider. Controlled and automatic processing during tasks requiring sustained attention. Human Factors, 23:737-750, 1981.

[77] T. S. for the Blind and V. impaired. http://www.tsbvi.edu/, Last checked: $01 / 26 / 2010$.

[78] W. A. for the Blind. http://www.worldaccessfortheblind.org/index.html, Last checked: $01 / 26 / 2010$. url.

[79] E. Foulk and J. Warm. Effects of complexity and redundancy on the tactual recognition of metric figures. Percept Mot Skills, 25:177-187, 1967.

[80] W. Fredrick. The use of classroom time in high schools above or below the median reading score. Urban Education, 11(4):459, 1977. 
[81] N. Funiyama. Gestural interaction between the instructor and the learner in origami instruction. Language and gesture, page 99, 2000.

[82] A. Furnham. The message, the context, and the medium. Language and Communication, 2:33-47, 1982.

[83] A. Gallace, H. Tan, and C. Spence. The body surface as a communication system: The state of the art after 50 years. PRESENCE: Teleoperators and Virtual Environments, 16(6):655-676, 2007.

[84] W. Gaver. The SonicFinder: An Interface That Uses Auditory Icons. Human-Computer Interaction, 4(1):67-94, 1989.

[85] W. Gaver, A. Sellen, C. Heath, and P. Luff. One is not enough: Multiple views in a media space. In Proceedings of the INTERACT'93 and CHI'93 conference on Human factors in computing systems, pages 335-341. ACM New York, NY, USA, 1993.

[86] F. Geldard. Some neglected possibilities of communication. Science, 131(3413):1583$1588,1960$.

[87] F. Geldard. Sensory Saltation: Metastability in the Perceptual World. Lawrence Erlbaum Associates, 1975.

[88] R. Geluykens. Tails (right-dislocations) as a repair mechanism in English conversation. Getting one's words into line: On word order andfunctional grammar. Dordrecht: Foris, pages 119-130, 1987.

[89] R. Geluykens. The Interactional Nature of Referent. Introduction. In CLS. Papers from the General Session at the... Regional Meeting, number 24-1, pages 141-154, 1988.

[90] G. Gescheider, S. Bolanowski, and R. Verrillo. Temporal relations in cutaneous stimulation. Cutaneous communication systems and devices, pages 33-37, 1974.

[91] J. Gibson. Observations on active touch. Psychological Review, 69(6):477-491, 1962.

[92] J. Gibson. A Direct Theory of Visual Perception. B e Psychology of kilowing, New York: Gordon and Breach, 1:979, 1972.

[93] W. Gibson and C. Darron. Teaching Statistics to a Student Who Is Blind. Teaching of Psychology, 26(2):130-131, 1999.

[94] K. Gilliland and R. Schlegel. Tactile Stimulation of the Human Head for Information Display. Human Factors: The Journal of the Human Factors and Ergonomics Society, 36(4):700-717, 1994.

[95] J. Godthelp and J. Schumann. Intelligent Accelerator: an Element of Driver Support. Driving future vehicles, pages 265-275, 1993. 
[96] G. Goff. Differential discrimination of frequency of cutaneous mechanical vibration. Journal of Experimental Psychology, 74(2):294, 1967.

[97] J. Goldberg. A system for the transfer of instructions in natural settings. Semiotica, 14(3):269-296, 1975.

[98] S. Goldin-Meadow. The role of gesture in communication and thinking. Trends in Cognitive Sciences, 3(11):419-429, 1999.

[99] S. Goldin-Meadow. Hearing Gesture: How Our Hands Help Us Think. Belknap Press, 2003.

[100] S. Goldin-Meadow and M. Singer. From Children's Hands to Adults' Ears: Gesture's Role in the Learning Process. DEVELOPMENTAL PSYCHOLOGY, 39(3):509-520, 2003.

[101] D. Grammenos, A. Savidis, Y. Georgalis, and C. Stephanidis. Access invaders: Developing a universally accessible action game. LECTURE NOTES IN COMPUTER SCIENCE, 4061:388, 2006.

[102] D. Grammenos, A. Savidis, and C. Stephanidis. UA-Chess: A universally accessible board game. In Proc. Of the $3 \mathrm{rd}$ Intl. Conf. Universal Access in Human-Computer Interaction. G. Salvendy (ed.), 2005.

[103] A. Grant, T. MC, and S. K. Tactile perception in blind braille readers: a psychophysical study of acuity and hyperacuity using gratings and dot patterns. Percept Psychophys, 62:301-312, 2000.

[104] D. Grant and V. Hayward. Variable structure control of shape memory alloy actuators. IEEE Control Systems Magazine, 17(3):80-88, 1997.

[105] Y. Guiard. Asymmetric division of labor in human skilled bimanual action: The kinematic chain as a model. Journal of Motor Behavior, 19(4):486-517, 1987.

[106] E. Gunther, G. Davenport, and S. O’Modhrain. Cutaneous grooves: composing for the sense of touch. In Proceedings of the 2002 conference on New interfaces for musical expression, pages 1-6. National University of Singapore Singapore, Singapore, 2002.

[107] R. HABER, L. HABER, C. LEVIN, and R. HOLLYFIELD. Properties of spatial representations: data from sighted and blind subjects. Perception $\&$ psychophysics, 54(1):1-13, 1993.

[108] J. Hadamard. The psychology of invention in the mathematical field. Princeton, NJ: Princeton, 1945. 
[109] K. S. Hale and K. M. Stanney. Deriving haptic design guidelines for human pshysiological, psychological and neurological foundations. IEEE Computer Graphics and Applications, 2004.

[110] Y. Hatwell, A. Streti, and E. Gentaz. Touching for knowing. Paris: Presses Universitaires de Frances, 2000.

[111] C. Heath, P. Luff, and A. Sellen. Reconsidering the virtual workplace: Flexible support for collaborative activity. In Proceedings of the fourth European conference on computer-supported cooperative work, pages 83-99. Springer, 1995.

[112] M. Heller. Tactile picture perception in sighted and blind people. Behavioural Brain Research, 135:65-68, 2002.

[113] M. Heller and J. Kennedy. Perspective taking, pictures, and the blind. Percept Psychophys, 48(5):459-66, 1990.

[114] M. Heller, J. Kennedy, and T. Joyner. Production and interpretation of pictures of houses by blind people. PERCEPTION-LONDON-, 24:1049-1049, 1995.

[115] M. A. Heller and W. Schiff. The Psychology of Touch. Lawrence Erlbaum Associates, 1991.

[116] J. Hennessy. Cutaneous sensitivity communications. Hum Factors, 8(5):463-9, 1966.

[117] J. Hill. Limited field of view in reading lettershapes with the fingers. Cutaneous communication systems and devices, pages 95-105, 1974.

[118] C. Ho, H. Tan, and C. Spence. Using spatial vibrotactile cues to direct a driver's visual attention. Transportation Research Part F: Traffic Psychology and Behaviour, 8:397-412, 2005.

[119] A. Hodzic, R. Veit, A. Karim, M. Erb, and B. Godde. Improvement and Decline in Tactile Discrimination Behavior after Cortical Plasticity Induced by Passive Tactile Coactivation. Journal of Neuroscience, 24(2):442, 2004.

[120] M. Hollins. Vision Impairment and Cognition. The Lighthouse Handbook on Vision Impairment and Vision Rehabilitation, 1:339-358, 2000.

[121] W. Horton and R. Gerrig. Speakers'experiences and audience design: Knowing when and knowing how to adjust utterances to addressees. Journal of Memory and Language, 47(4):589-606, 2002.

[122] Humanware. Humanware. http://www.hmw.it/prodotti_e.html, Last checked: 01/26/2010, 112009. 
[123] R. Iglesias, S. Casado, T. Gutierrez, J. Barbero, C. Avizzano, S. Marcheschi, M. Bergamasco, F. Labein, and S. Derio. Computer graphics access for blind people through a haptic and audio virtual environment. In Haptic, Audio and Visual Environments and Their Applications, 2004. HAVE 2004. Proceedings. The 3rd IEEE International Workshop on, pages 13-18, 2004.

[124] D. Inman, K. Loge, and A. Cram. Teaching orientation and mobility skills to blind children using computer generated 3-d sound environments. Proceedings of the International Community for Auditory Display, Atlanta Georgia, April, 2000.

[125] D. Inman, J. Peaks, K. Loge, V. Chen, and G. Ferrington. Virtual Reality Training Program for Motorized Wheelchair Operation. California State University, Northridge Center on Disabilities, Los Angeles, 1994.

[126] C. Intel. Open cv, 05/30/2007 2006.

[127] S. Ishiai. What do eye-fixation patterns tell us about unilateral spatial neglect? Restorative Neurology and Neuroscience, 24(4):261-271, 2006.

[128] J. Iverson. How to get to the cafeteria: gesture and speech in blind and sighted children's spatial descriptions. Developmental psychology, 35(4):1132-1142, 1999.

[129] J. Iverson and S. Goldin-Meadow. What's communication got to do with it? Gesture in children blind from birth. Developmental Psychology, 33(3):453-467, 1997.

[130] J. Iverson and S. Goldin-Meadow. Why people gesture as they speak. Nature, 396:228, 1998.

[131] E. Jacobson. ELECTRICAL MEASUREMENTS OF NEUROMUSCULAR STATES DURING MENTAL ACTIVITIES I. Imagination of Movement Involving Skeletal Muscle. American journal of Physiology, 91(2):567-608, 1930.

[132] W. James. The principles of psychology. New York: Holt, 1890.

[133] W. Janssen and L. Nilsson. Behavioural effects of driver support. Driving future vehicles, pages 147-155, 1993.

[134] G. Jansson and L. Monaci. Identification of real objects under conditions similar to those in haptic displays: providing spatially distributed information at the contact areas is more important than increasing the number of areas. Virtual Reality, 9(4):243$249,2006$.

[135] G. Jansson and P. Pedersen. Obtaining geographical information from a virtual map with a haptic mouse. In XXII International Cartographic Conference (ICC2005), 2005. 
[136] C. Jay, M. Glencross, and R. Hubbold. Modeling the effects of delayed haptic and visual feedback in a collaborative virtual environment. Transactions on ComputerHuman Interaction, 14(2), 2007.

[137] M. Jeannerod. The representing brain: Neural correlates of motor intention and imagery. BEHAVIORAL AND BRAIN SCIENCES, 17:187-187, 1994.

[138] G. Jefferson. Preliminary notes on a possible metric which provides for a 'standard maximum'silence of approximately one second in conversation. In. Roger, Derek. Bull, Peter (eds.): Conversation: An interdisciplinary perspective. Clevedon, S, pages 166$196,1989$.

[139] C. Jenova. Flow in games. PhD thesis, University of Southern California, 2001.

[140] W. Joeong and M. Gluck. Multimodal bivariate thematic maps with auditory and haptic display. In Proceedings of the 2002 International Conference on Auditory Display, Kyoto, Japan, 2002.

[141] A. Johansson and J. Linde. Using simple force feedback mechanisms as haptic visualizationtools. In Instrumentation and Measurement Technology Conference, 1999. IMTC/99. Proceedings of the 16th IEEE, volume 2, 1999.

[142] D. Johnson and J. Wiles. Effective affective user interface design in games. Ergonomics, 46(13):1332-1345, 2003.

[143] K. Johnson and J. Phillips. Tactile spatial resolution. I. Two-point discrimination, gap detection, grating resolution, and letter recognition. Journal of Neurophysiology, 46(6):1177-1192, 1981.

[144] L. Jones and M. Berris. Material discrimination and thermal perception. In Haptic Interfaces for Virtual Environment and Teleoperator Systems, 2003. HAPTICS 2003. Proceedings. 11th Symposium on, pages 171-178, 2003.

[145] L. Jones, B. Lockyer, and E. Piateski. Tactile display and vibrotactile pattern recognition on the torso. Advanced Robotics, 20(12):1359-1374, 2006.

[146] R. Jonhansson and A. Valbo. Tactile sensibility in the human hand: relative and absolute densities of four types of mechanoreceptive units in glabrous skin. The Journal of Physiology, 286:283-300, 1979.

[147] M. Jonhson. The body in the mind. University of Chicago Press, 1987.

[148] K. KACZMAREK and J. WEBSTER. Electrotactile and vibrotactile displays for sensory substitution systems. IEEE Transactions on Biomedical Engineering, 38:1-16, 1991. 
[149] S. KALYUGA, P. CHANDLER, and J. SWELLER. Managing split-attention and redundancy in multimedia instruction. Applied Cognitive Psychology, 13:351-371, 1999.

[150] L. Karsenty. Cooperative work and shared visual context: An empirical study of comprehension problems in side-by-side and remote help dialogues. Human-Computer Interaction, 14(3):283-315, 1999.

[151] S. Kelly, M. Singer, J. Hicks, and S. Goldin-Meadow. A Helping Hand in Assessing Children's Knowledge: Instructing Adults to Attend to Gesture. Cognition and Instruction, 20(1):1-26, 2002.

[152] A. Kendon. How gestures can become like words. In Cross-Cultural Persperctives in Nonverbal Communication. Hogrede, 1988.

[153] A. Kendon. Sign languages of aboriginal Australia: cultural, semiotic and communicative perspectives. Cambridge University Press, 1988.

[154] A. Kendon. Gesture: visible action as utterance, 2004.

[155] J. M. Kennedy. Drawing and the Blind. Yale Press, New Haven, 1993.

[156] N. Kerr. The role of vision in" visual imagery" experiments: evidence from the congenitally blind. J Exp Psychol Gen, 112(2):265-77, 1983.

[157] I. Kimbara. On gestural mimicry. Gesture, 6(1):39-61, 2006.

[158] J. Kirman. Tactile apparent movement- The effects of interstimulus onset interval and stimulus duration. Perception and Psychophysics, 15:1-6, 1974.

[159] R. Klatzky, J. Loomis, S. Lederman, H. Wake, and N. Fujita. Haptic identification of objects and their depictions. PERCEPTION AND PSYCHOPHYSICS, 54:170-170, 1993.

[160] K. Kokjer. The information capacity of the human fingertip. IEEE Transactions on Systems, Man and Cybernetics, 17(1):100-102, 1987.

[161] S. Kosslyn, T. Ball, and B. Reiser. Visual images preserve metric spatial information: Evidence from studies of image scanning. Journal of Experimental Psychology: Human Perception and Performance, 4(1):47-60, 1978.

[162] R. Kraut, S. Fussell, and J. Siegel. Visual information as a conversational resource in collaborative physical tasks. Human-computer interaction, 18(1):13-49, 2003.

[163] R. Kraut, D. Gergle, and S. Fussell. The use of visual information in shared visual spaces: Informing the development of virtual co-presence. In Proceedings of the 2002 ACM conference on Computer supported cooperative work, pages 31-40. ACM New York, NY, USA, 2002. 
[164] R. E. Kraut, M. Miller, and J. Seigel. Collaboration in performance of physical tasks: effects on outcomes and communication. In Proceedings of CSCW 1996, ACM Conference on Computer-Supported Cooperative Work, 1996.

[165] Y. Kume, A. Shirai, M. Tsuda, and T. Hatada. Information transmission through soles by vibrotactile stimulation. Trans. of the Virtual Reality Society of Japan, 3(3):83-88, 1998.

[166] D. LaBerge. Attention and the measurement of perceptual learning. Memory and Cognition, 1:268-276, 1973.

[167] G. Lakoff. Women, Fire and Dangerous things: What categories Reveal about mind. University of Chicago Press, 1987.

[168] G. Lakoff and M. Johnson. Philosophy in the Flesh: The Embodied Mind and Its Challenge to Western Thought. Basic Books, 1999.

[169] lécuyer2003homere. Homere: a multimodal system for visually impaired people to explore virtual environments. IEEE Virtual Reality, 2003. Proceedings, pages 251$258,2003$.

[170] J. Lee, J. Hoffman, and E. Hayes. Collision warning design to mitigate driver distraction. In Proceedings of the SIGCHI conference on Human factors in computing systems, pages 65-72. ACM Press New York, NY, USA, 2004.

[171] R. Lindeman, Y. Yanagida, J. Sibert, and R. Lavine. Effective Vibrotactile Cueing in a Visual Search Task. Human-Computer Interaction, 2003.

[172] D. LLOYD, N. MERAT, F. Mc GLONE, and C. SPENCE. Crossmodal links between audition and touch in covert endogenous spatial attention. Perception $\& 3$ Psychophysics, 65(6):901-924, 2003.

[173] G. L. Lohse. Models of graphical perception. In M. Helander, editor, Handbook of Human-Computer Interaction, pages 107-135, 1997.

[174] J. Loomis, R. Klatzky, and S. Lederman. Similarity of tactual and visual picture recognition with limited field of view. Perception, 20(2):167-177, 1991.

[175] B. Lucas and T. Kanade. An iterative image registration technique with an application to stereo vision. In Proc. of 7th International Joint Conference on Artificial Intelligence, pages 674-679, 1986.

[176] P. Luff, C. Heath, and D. Greatbatch. Tasks-in-interaction: paper and screen based documentation in collaborative activity. In Proceedings of the 1992 ACM conference on Computer-supported cooperative work, pages 163-170. ACM New York, NY, USA, 1992. 
[177] I. MacKenzie. Motor behaviour models for human-computer interaction. HCI Models, Theories, and Frameworks-Toward a Multidisciplinary Science, pages 27-54, 2003.

[178] L. Magee and J. Kennedy. Exploring pictures tactually. Nature, 283(5744):287-288, 1980.

[179] M. Mahoney and M. Avener. Psychology of the elite athlete: An exploratory study. Cognitive Therapy and Research, 1(2):135-141, 1977.

[180] M. Manshad and A. Manshad. Multimodal vision glove for touchscreens. In Proceedings of the 10th international ACM SIGACCESS conference on Computers and accessibility, pages 251-252. ACM New York, NY, USA, 2008.

[181] C. Marshall. Toward an ecology of hypertext annotation. Proceedings of the ninth ACM conference on Hypertext and hypermedia: links, objects, time and space-structure in hypermedia systems: links, objects, time and space-structure in hypermedia systems, pages 40-49, 1998.

[182] C. J. McCallister and R. L. Kennedy. Teaching basic statistics to a student who is blind. In Annual Meeting of the Mid-South Educational Research Association, November 2001.

[183] J. McCarthy, V. Miles, A. Monk, M. Harrison, A. Dix, and P. Wright. Text-based on-line conferencing: a conceptual and empirical analysis using a minimal prototype. Human-Computer Interaction, 8(2):147-183, 1993.

[184] J. McElligott and L. Van Leeuwen. Designing sound tools and toys for blind and visually impaired children. In Proceedings of the 2004 conference on Interaction design and children: building a community, pages 65-72. ACM New York, NY, USA, 2004.

[185] D. McGehee, M. Raby, J. Lee, and G. Nourse. Final design and operating characteristics of a snowplow lane awareness system. In 80th Annual Meeting of the Transportation Research Board, pages 7-11, 2001.

[186] D. McGookin and S. Brewster. Contextual audio in haptic graph browsing. In proceedings of ICAD, pages 91-94, 2006.

[187] D. McGookin and S. Brewster. Multivis: improving access to visualisations for visually impaired people. In CHI '06: $\mathrm{CHI}$ '06 extended abstracts on Human factors in computing systems, pages 267-270. ACM, 2006.

[188] D. McNeill. Psycholinguistics: A New Approach. Harpercollins College Div, 1987.

[189] D. McNeill. Hand and Mind: What Gestures Reveal about thought. University of Chicago Press, Chicago, IL, 1992.

[190] D. McNeill. Gesture and Thought. University of Chicago Press, 2005. 
[191] D. McNeill. Gesture, Gaze, and Ground. LECTURE NOTES IN COMPUTER SCIENCE, 3869:1, 2006.

[192] D. MCNEILL, J. CASSELL, and E. LEVY. Abstract deixis. Semiotica, 95(1-2):5-19, 1993.

[193] G. Mead. Mind, Self and Society from the standpoint of a social behaviorist. University of Chicago Press, 1974.

[194] G. Miller. The magical number of seven, plus or minus two: Some limits on our capacity for processing information. Psychological Review, 63(2):81-86, 1956.

[195] A. Monk. Common ground in electronically mediated communication. In M. kaufman, editor, HCI Models, Theories, and Frameworks: Toward a Multidisciplinary Science, pages 265-286. Morgan Kaufmann Pub, San Francisco, 2003.

[196] G. Monkman, P. Taylor, F. Elektronik, and F. Regensburg. Thermal tactile sensing. Robotics and Automation, IEEE Transactions on, 9(3):313-318, 1993.

[197] A. Mulder and S. Fels. Sound Sculpting: Manipulating sound through virtual sculpting. In Proc. of the 1998 Western Computer Graphics Symposium, pages 15-23, 1998.

[198] N. H. Museum. Sensory homunculus. http://piclib.nhm.ac.uk/piclib/www/ image . php?img=87494, Last checked: $01 / 26 / 2010$.

[199] E. Mynatt and G. Wber. Nonvisual presentation of graphical user interfaces. In A. Press, editor, Proceedings of CHI 1994, 1994.

[200] B. Nardi, A. Kuchinsky, S. Whittaker, R. Leichner, and H. Schwarz. Video-as-data: Technical and social aspects of a collaborative multimedia application. Computer Supported Cooperative Work (CSCW), 4(1):73-100, 1995.

[201] B. A. Nardi. Concepts of cognition and consciousness: Four voices. Journal of Computer Documentation, 22(1), 1998.

[202] L. Narici. Neuromagnetic somatosensory homunculus: A non-invasive approach to humans. Neuroscience Letters, 121:51-54, 1991.

[203] U. Nations. Enable. http://www.un.org/esa/socdev/enable/diswpa04.htm, Last checked: $01 / 26 / 2010$.

[204] F. Oliveira and F. Quek. A multimodal communication with a haptic glove: On the fusion of speech and deictic over a raised line drawing. In Petra - The 1st International Conference on PErvasive Technologies Related to Assistive Environments, 2008.

[205] R. Ossmann and K. Miesenberger. Guidelines for the development of accessible computer games. Lecture Notes in Computer Science, 4061:403, 2006. 
[206] S. A. Osterhaus. Thoughts on visual vs tactual perception. http://www.tsbvi.edu/ math/mi-04.htm, Last checked: 01/26/2010, November 2004.

[207] O. Otto and D. Roberts. Importance of communication on a highly collaborative task. In Proceedings of the Seventh IEEE International Symposium on Distributed Simulation and Real-Time Applications, 2003.

[208] S. Oviatt. Mutual disambiguation of recognition errors in a multimodel architecture. In Proceedings of the SIGCHI conference on Human factors in computing systems: the CHI is the limit, pages 576-583. ACM New York, NY, USA, 1999.

[209] S. Oviatt and P. Cohen. Discourse structure and performance efficiency in interactive and non-interactive spoken modalities. Computer speech $\&$ language(Print), 5(4):297326, 1991.

[210] T. Paek. Toward a Taxonomy of Communication Errors. ISCA Tutorial and Research Workshop on Error Handling in Spoken Dialogue Systems, 2003.

[211] A. Pascual-Leone and F. Torres. Plasticity of the sensorimotor cortex representation of the reading finger in braille readers. Brain, 116(1):2230-2236, 1993.

[212] R. Penrose. The Emperor's new mind. Oxford University Press, New York, 1989.

[213] L. Petrosino and D. Fucci. Temporal resolution of the aging tactile sensory system. Perceptual and motor skills, 68(1):288, 1989.

[214] S. Pinker. A theory of graph comprehension. In Artificial Intelligence and the Future of Testing, 1990.

[215] C. Prablanc, J. Echallier, E. Komilis, and M. Jeannerod. Optimal response of eye and hand motor systems in pointing at a visual target. I. Spatio-temporal characteristics of eye and hand movements and their relationships when varying the amount of visual information. Biol Cybern, 35(2):113-124, 1979.

[216] A. Puret, D. Archambault, N. Monmarché, and M. Slimane. A simple game generator for creating audio/tactile games. Technology and Disability, 18(4):227-236, 2006.

[217] F. Quek. The catchment feature model: A device for multimodal fusion and a bridge between signal and sense. EURASIP Journal on Applied Signal Processing, pages 1619-1636, 2004.

[218] F. Quek, D. McNeill, and F. Oliveira. Enabling multimodal communications for enhancing the ability of learning for the visually impaired. In ICMI '06: Proceedings of the 8th international conference on Multimodal interfaces, pages 326-332. ACM, 2006.

[219] L. Radford. Why do gestures matter? Gestures as semiotic means of objectification. In PME CONFERENCE, volume 29, page 1, 2005. 
[220] R. Raisamo, S. Patomaki, M. Hasu, and V. Pasto. Design and evaluation of a tactile memory game for visually impaired children. Interacting with Computers, 19(2):196205, 2007.

[221] V. Ramachandran and S. Blakeslee. Phantoms in the Brain: Probing the Misteries of the Human Mind. William Morrow, 1998.

[222] H. Regenbrecht, J. Hauber, R. Schoenfelder, and A. Maegerlein. Virtual reality aided assembly with directional vibro-tactile feedback. In Proceedings of the 3rd international conference on Computer graphics and interactive techniques in Australasia and South East Asia, pages 381-387. ACM New York, NY, USA, 2005.

[223] G. a. Reicher. Perceptual recognition as a function of meaningfulness of stimulus material. Human Performance Center, Dept. of Psychology, University of Michigan, 1968.

[224] A. Reid. Comparing the telephone with face-to-face interaction. The social impact of the telephone, pages 386-414, 1977.

[225] B. Riedel and A. Burton. Perception of gradient in haptic graphs: a comparison of virtual and physical stimuli. In Eurohaptics 2001 Conference Proceedings Birmingham $U K$, pages 90-92, 2001.

[226] G. Rizzolatti and M. Arbib. Language within our grasp. Trends in Neurosciences, 21(5):188-194, 1998.

[227] R. Rose, F. Quek, and Y. Shi. MacVisSTA: a system for multimodal analysis. In Proceedings of the 6th international conference on Multimodal interfaces, pages 259264. ACM New York, NY, USA, 2004.

[228] P. Roth, L. Petrucci, A. Assimacopoulos, and T. Pun. Audio-haptic internet browser and associated tools for blind and visually impaired computer users. In Workshop on friendly exchanging through the net March, volume 22, page 24, 2000.

[229] P. Roth, L. Petrucci, T. Pun, and A. Assimacopoulos. Auditory browser for blind and visually impaired users. In CHI '99: CHI'99 extended abstracts on Human factors in computing systems, pages 218-219, New York, NY, USA, 1999. ACM.

[230] D. Rumelhart. Human Information Processing. John Wiley, 1977.

[231] A. Rupert. Which way is down. Naval Aviation News, pages 16-17, 1997.

[232] G. Salvendy. Handbook of human factors and ergonomics. Wiley New York, 1997.

[233] J. Sánchez and H. Flores. Audiomath: Blind children learning mathematics through audio. International Journal on Disability and Human Development, 4(4):311, 2005. 
[234] K. Sathian. Practice makes perfect: Sharper tactile perception in the blind. Neurology, 54(12):Editorial, 2000.

[235] W. Schiff and E. Foulke. Tactual Perception: A Sourcebook. Cambridge University Press, 1982.

[236] R. Schmidt. Somatovisceral sensibility. Fundamentals of sensory physiology, 1978.

[237] J. Schumann, H. Godthelp, B. Farber, H. Wontorra, A. Gale, I. Brown, C. Haslegrave, H. Kruysse, and S. Taylor. Breaking Up Open-loop Steering Control Actions: The Steering Wheel as an Active Control Device. North-Holland, 1993.

[238] F. Scientific. http://www.freedomscientific.com/products/fs/ jaws-product-page.asp, Last checked: 01/26/2010.

[239] H. Segond, D. Weiss, and E. Sampaio. Human spatial navigation via a visuo-tactile sensory substitution system. Perception, 34(10):1231-1249, 2005.

[240] A. Sellen. Remote Conversations: The Effects of Mediating Talk with Technology. Human-Computer Interaction, 10(4):401-444, 1995.

[241] A. Sellen and R. Harper. The myth of the paperless office. MIT press Cambridge, Mass, 2002.

[242] Sensable. http://www. sensable.com/products-haptic-devices.htm, Last checked: $01 / 26 / 2010$.

[243] A. Sepchat, N. Monmarche, M. Slimane, and D. Archambault. Semi automatic generator of tactile video games for visually impaired children. LECTURE NOTES IN COMPUTER SCIENCE, 4061:372, 2006.

[244] C. Sherrick. Touch as a communicative sense: Introduction. The Journal of the Acoustical Society of America, 77:218, 1985.

[245] J. Sherzer. Verbal and Nonverbal Deixis: The Pointed Lip Gesture among the San Blas Cuna. Language in Society, 2(1):117-131, 1973.

[246] J. Short, E. Williams, and B. Christie. The social psychology of telecommunications. John Wiley \& Sons, 1976.

[247] C. Sjostrom. Designing haptic computer interfaces for blind people. Proc. ISSPA 2001, pages 1-4, 2001.

[248] N. Smith. Gesture and beyond. University of California at Berkely, 2003.

[249] sourceforge.net. Vtplayer. Internet. 
[250] C. Spence, M. Nicholls, and J. Driver. The cost of expecting events in the wrong sensory modality. Perception \& Psychophysics, 63(2):330-336, 2001.

[251] C. Spence, D. Shore, and R. Klein. Multisensory Prior Entry. JOURNAL OF EXPERIMENTAL PSYCHOLOGY GENERAL, 130(4):799-832, 2001.

[252] R. Splindler. Teaching mathematics to a student who is blind. Teaching Mathematics and its applications, 25(3), 2005.

[253] B. Stallings. Training busy staff to succeed with volunteers: Building commitment and competence in staff/volunteer teams, 1998.

[254] D. Staub and C. Peck. What are the outcomes for nondisabled students? Educational Leadership, 52(4):36-40, 1994.

[255] J. Stevens, E. Foulk, and M. Patterson. Tactile acuity, aging and braille reading in long-term blindness. J Exp Psychol Applied, 2:91-96, 1996.

[256] S. Stinson. Body of knowledge. Education and Society, 45(1):43-55, 1995.

[257] C. Supalo. Techniques to enhance instructors' teaching effectiveness with chemistry students who are blind or visually impaired. Journal of Chemical Education, 82(10):1513, 2005.

[258] D. Tall. A theory of mathematical growth through embodiment, symbolism and proof. In International Colloquium on Mathematical Learning from Early Childhood to Adulthood., Nevellis, Belguim, 2005.

[259] H. Tan, A. Lim, and R. Traylor. A psychophysical study of sensory saltation with an open response paradigm. In Proc. 9th Int'l Symp. Haptic Interfaces for Virtual Environment and Teleoperator Systems, 2000.

[260] H. Tan, M. Srinivasan, B. Eberman, and B. Cheng. Human Factors for the Design of Force-Reflecting Haptic Interfaces. Tan, Srinivasan, Eberman, $\&$ Chang, ASME WAM, pages 1-11, 1994.

[261] D. G. Tatar, G. Foster, and D. Bobrow. Designing for conversation: Lessons from cognoter. International Journal of Man-machine studies, 34:185-209, 1991.

[262] N. Tye-Murray and S. Witt. Conversational moves and conversational styles of adult cochlear-implant users. JOURNAL-ACADEMY OF REHABILITATIVE AUDIOL$O G Y, 29: 11-26,1996$.

[263] O. v. Clementon. Court decision, 1993. 995 F.2D 1204, (3rd Cir. 1993).

[264] R. Van Boven, R. Hamilton, T. Kauffman, J. Keenan, and A. Pascual-Leone. Tactile spatial resolution in blind braille readers. Neurology, 54:2230-2236, 2000. 
[265] J. van Erp. Guidelines for the use of vibro-tactile displays in human computer interaction. In Proceedings of Eurohaptics 2002, pages 18-22, 2002.

[266] J. van Erp and H. van Veen. A multipurpose tactile vest for astronauts in the international space station. In Proceedings of Eurohaptics, pages 405-408, 2003.

[267] J. Van Erp, H. Van Veen, C. Jansen, and T. Dobbins. Waypoint navigation with a vibrotactile waist belt. ACM Transactions on Applied Perception (TAP), 2(2):106-117, 2005.

[268] F. Van Scoy, D. McLaughlin, and A. Fullmer. Auditory augmentation of haptic graphs: Developing a graphic tool for teaching precalculus skill to blind students. Proc. AGS 2005, 2005.

[269] H. van Veen and J. van Erp. Tactile Information Presentation in the Cockpit. LECTURE NOTES IN COMPUTER SCIENCE, pages 174-181, 2001.

[270] E. Veinott, J. O. G. Olson, and X. Fu. Video helps remote work. In Proceedings of CHI 1999, ACM Conference on Computer-Human Interaction, 1999.

[271] E. Velleman, R. van Tol, S. Huiberts, and H. Verwey. 3d shooting games, multimodal games, sound games and more working examples of the future of games for the blind. Development, 2002, 2001.

[272] R. Verrillo. Temporal summation in vibrotactile sensitivity. The Journal of the Acoustical Society of America, 37:843, 1965.

[273] R. Verrillo and G. Gescheider. Enhancement and summation in the perception of two successive vibrotactile stimuli. Percept Psychophys, 18:128-136, 1975.

[274] R. Verrillo and G. Gescheider. Perception via the sense of touch. Tactile Aids for the Hearing Impaired, pages 1-36, 1992.

[275] H. VITENSE, J. JACKO, and V. EMERY. Multimodal feedback: an assessment of performance and mental workload. Ergonomics, 46(1-3):68-87, 2003.

[276] L. Vygostky. Mind and Society. Harvard University Press, 1978.

[277] C. Wagner, S. Lederman, and R. Howe. A tactile shape display using RC servomotors. In Haptic Interfaces for Virtual Environment and Teleoperator Systems, 2002. HAPTICS 2002. Proceedings. 10th Symposium on, pages 354-355, 2002.

[278] C. Wagner, S. Lederman, and R. Howe. Design and performance of a tactile shape display using rc servomotors. Haptics-e, 3(4), 2004.

[279] S. Wall and S. Brewster. Sensory substitution using tactile pin arrays: Human factors, technology and applications. Signal Processing, 86(12):3674-3695, 2006. 
[280] S. Wall and S. Brewster. Tac-tiles: multimodal pie charts for visually impaired users. In Proceedings of the 4th Nordic conference on Human-computer interaction: changing roles, pages 9-18. ACM Press New York, NY, USA, 2006.

[281] Q. Wang and V. Hayward. Compact, portable, modular, high-performance, distributed tactile transducer device based on lateral skin deformation. In Haptic Interfaces for Virtual Environment and Teleoperator Systems, 2006 14th Symposium on, pages 67-72, 2006.

[282] S. WEINSTEIN. Intensive and extensive aspects of tactile sensitivity as a function. In The Skin Senses: Proceedings. Thomas, 1968.

[283] L. Wells and S. Landau. Merging of tactile sensory input and audio data by means of the talking tactile tablet. In Eurohaptics 2003, pages 414-418, 2003.

[284] T. Westin. Game accessibility case study: Terraformers-a real-time 3D graphic game. In The Fifth International Conference on Disability, Virtual Reality and Associated Technologies, 2004.

[285] S. Whittaker. Rethinking video as a technology for interpersonal communications: theory and design implications. International Journal of Human-Computer Studies, 42(5):501-529, 1995.

[286] S. Whittaker. Things to talk about when talking about things. Human-Computer Interaction, 18(1):149-170, 2003.

[287] S. Whittaker, S. Brennan, and H. Clark. Co-ordinating activity: an analysis of interaction in computer-supported co-operative work. In Proceedings of the SIGCHI conference on Human factors in computing systems: Reaching through technology, pages 361-367. ACM New York, NY, USA, 1991.

[288] S. Whittaker, D. Frohlich, and O. Daly-Jones. Informal workplace communication: What is it like and how might we support it? In Proceedings of the SIGCHI conference on Human factors in computing systems: celebrating interdependence, pages 131-137. ACM New York, NY, USA, 1994.

[289] S. Whittaker, E. Geelhoed, and E. Robinson. Shared workspaces: how do they work and when are they useful? International Journal of Man-Machine Studies, 39(5):813$842,1993$.

[290] S. Whittaker and B. O'Conaill. The role of vision in face-to-face and mediated communication. Video-mediated communication, pages 23-49, 1997.

[291] C. Wickens and J. Hollands. Engineering Psychology and Human Performance. Prentice Hall, 2001. 
[292] A. Wiliams, E. Kabish, and P. Dourish. From interaction to participation: Configuring space through embodied interaction. In in Proc. Intl. Conf. Ubiquitous Computing (Ubicomp), Tokyo, Japan, 2005.

[293] D. Wilkins. Why pointing with the index finger is not a universal (in sociocultural and semiotic terms). Pointing: Where language, culture, and cognition meet, pages $171-215,2003$.

[294] A. Williams, E. Kabish, and P. Dourish. From interaction to participation: Configuring space through embodied interaction. In Proc. Intl. Conf. Ubiquitous Computing Ubicomp 2005, 2005.

[295] J. M. Williams. Nationwide shortage of teachers for blind students must be corrected. National Federation of the Blind: Advocates for Equality, Canadian Blind Monitor, 2002.

[296] M. Wilson. Six views of embodied cognition. Psychnomic bulletin and review, 9(4):625$636,2002$.

[297] F. Winberg and J. Bowers. Assembling the senses: towards the design of cooperative interfaces for visually impaired users. In $C S C W$ '04: Proceedings of the 2004 $A C M$ conference on Computer supported cooperative work, pages 332-341. ACM, 2004.

[298] B. Witmer and M. Singer. Measuring presence in virtual environments: A presence questionnaire. Presence, 7(3):225-240, 1998.

[299] A. Yarbus. Eye Movements and Vision. Plenum Press, 1967.

[300] W. Yu and S. Brewster. Comparing Two Haptic Interfaces for Multimodal Graph Rendering. In Proceedings of the 10th IEEE Symposium on Haptic Interfaces for Virtual Environment and Teleoperator Systems, IEEE, Orlando, Florida, USA, 2002.

[301] B. Yuan and E. Folmer. Blind hero: enabling guitar hero for the visually impaired. In Proceedings of the 10th international ACM SIGACCESS conference on Computers and accessibility, pages 169-176. ACM New York, NY, USA, 2008.

[302] J. Zelek. A haptic glove as a tactile-vision sensory substitution for wayfinding. Journal of Visual Impairment \&3 Blindness (JVIB), 97(10), 2003.

[303] J. Zelek. Seeing by touch (haptics) for wayfinding. In International Congress Series, volume 1282, pages 1108-1112. Elsevier, 2005. 
Appendix A

Chapter 2 - Additional Material 

A.1 Confusion Matrix 
Table A.1: Confusion Matrix

\begin{tabular}{|c|c|c|c|c|c|c|c|c|c|c|}
\hline \multirow{2}{*}{$\begin{array}{l}\text { Glove } \\
\text { Model }\end{array}$} & \multirow{2}{*}{$\begin{array}{l}\text { Direction } \\
\text { Sent }\end{array}$} & \multicolumn{9}{|c|}{ Direction Percieved } \\
\hline & & $\mathrm{N}$ & $\mathrm{NE}$ & $\mathrm{E}$ & $\mathrm{SE}$ & $\mathrm{S}$ & SW & $\mathrm{W}$ & NW & Total \\
\hline $5 \times 4 \mathrm{DH}$ & $\mathrm{N}$ & 1 & 3 & 0 & 0 & 0 & 0 & 0 & 0 & 4 \\
\hline $5 \times 4 \mathrm{NDH}$ & $\mathrm{N}$ & 3 & 0 & 0 & 0 & 0 & 0 & 0 & 1 & 4 \\
\hline $4 \times 4 \mathrm{DH}$ & $\mathrm{N}$ & 4 & 1 & 0 & 0 & 0 & 0 & 0 & 0 & 5 \\
\hline $4 \times 4 \mathrm{NDH}$ & $\mathrm{N}$ & 4 & 0 & 0 & 0 & 0 & 0 & 0 & 0 & 4 \\
\hline Round DH & $\mathrm{N}$ & 1 & 0 & 2 & 0 & 0 & 0 & 0 & 1 & 4 \\
\hline Round NDH & $\mathrm{N}$ & 1 & 0 & 0 & 0 & 0 & 0 & 2 & 1 & 4 \\
\hline $5 \times 4 \mathrm{DH}$ & $\mathrm{NE}$ & 0 & 4 & 0 & 0 & 0 & 0 & 0 & 0 & 4 \\
\hline $5 \times 4 \mathrm{NDH}$ & $\mathrm{NE}$ & 2 & 2 & 0 & 0 & 0 & 0 & 0 & 0 & 4 \\
\hline $4 \mathrm{x} 4 \mathrm{DH}$ & $\mathrm{NE}$ & 1 & 4 & 0 & 0 & 0 & 0 & 0 & 0 & 5 \\
\hline $4 \mathrm{x} 4 \mathrm{NDH}$ & $\mathrm{NE}$ & 1 & 3 & 0 & 0 & 0 & 0 & 0 & 0 & 4 \\
\hline Round DH & $\mathrm{NE}$ & 0 & 1 & 2 & 0 & 1 & 0 & 0 & 0 & 4 \\
\hline Round NDH & $\mathrm{NE}$ & 0 & 3 & 0 & 1 & 0 & 0 & 0 & 0 & 4 \\
\hline $5 \times 4 \mathrm{DH}$ & $\mathrm{E}$ & 0 & 0 & 4 & 0 & 0 & 0 & 0 & 0 & 4 \\
\hline $5 \times 4 \mathrm{NDH}$ & $\mathrm{E}$ & 0 & 0 & 3 & 0 & 1 & 0 & 0 & 0 & 4 \\
\hline $4 \times 4 \mathrm{DH}$ & $\mathrm{E}$ & 0 & 1 & 4 & 0 & 0 & 0 & 0 & 0 & 5 \\
\hline $4 \times 4 \mathrm{NDH}$ & $\mathrm{E}$ & 0 & 0 & 4 & 0 & 0 & 0 & 0 & 0 & 4 \\
\hline Round DH & $\mathrm{E}$ & 0 & 0 & 1 & 1 & 2 & 0 & 0 & 0 & 4 \\
\hline Round NDH & $\mathrm{E}$ & 0 & 1 & 3 & 0 & 0 & 0 & 0 & 0 & 4 \\
\hline $5 \times 4 \mathrm{DH}$ & $\mathrm{SE}$ & 0 & 0 & 0 & 4 & 0 & 0 & 0 & 0 & 4 \\
\hline $5 \times 4 \mathrm{NDH}$ & $\mathrm{SE}$ & 0 & 0 & 0 & 2 & 2 & 0 & 0 & 0 & 4 \\
\hline $4 \times 4 \mathrm{DH}$ & $\mathrm{SE}$ & 0 & 0 & 0 & 5 & 0 & 0 & 0 & 0 & 5 \\
\hline $4 \times 4 \mathrm{NDH}$ & $\mathrm{SE}$ & 0 & 0 & 0 & 4 & 0 & 0 & 0 & 0 & 4 \\
\hline Round DH & $\mathrm{SE}$ & 0 & 0 & 1 & 1 & 2 & 0 & 0 & 0 & 4 \\
\hline Round NDH & $\mathrm{SE}$ & 0 & 0 & 0 & 3 & 1 & 0 & 0 & 0 & 4 \\
\hline $5 \times 4 \mathrm{DH}$ & $\mathrm{S}$ & 0 & 0 & 0 & 0 & 3 & 1 & 0 & 0 & 4 \\
\hline $5 \times 4 \mathrm{NDH}$ & $\mathrm{S}$ & 0 & 0 & 0 & 0 & 3 & 0 & 1 & 0 & 4 \\
\hline $4 \times 4 \mathrm{DH}$ & $\mathrm{S}$ & 0 & 0 & 0 & 1 & 4 & 0 & 0 & 0 & 5 \\
\hline $4 \mathrm{x} 4 \mathrm{NDH}$ & $\mathrm{S}$ & 0 & 0 & 0 & 1 & 3 & 0 & 0 & 0 & 4 \\
\hline Round DH & $\mathrm{S}$ & 1 & 0 & 0 & 2 & 1 & 0 & 0 & 0 & 4 \\
\hline Round NDH & $\mathrm{S}$ & 0 & 0 & 0 & 0 & 4 & 0 & 0 & 0 & 4 \\
\hline $5 \times 4 \mathrm{DH}$ & SW & 0 & 0 & 0 & 0 & 0 & 4 & 0 & 0 & 4 \\
\hline $5 \times 4 \mathrm{NDH}$ & SW & 0 & 0 & 0 & 0 & 0 & 4 & 0 & 0 & 4 \\
\hline $4 \times 4 \mathrm{DH}$ & SW & 0 & 0 & 0 & 0 & 0 & 5 & 0 & 0 & 5 \\
\hline $4 \times 4 \mathrm{NDH}$ & SW & 0 & 0 & 0 & 0 & 3 & 1 & 0 & 0 & 4 \\
\hline Round DH & SW & 0 & 0 & 0 & 0 & 1 & 3 & 0 & 0 & 4 \\
\hline Round NDH & SW & 0 & 0 & 0 & 0 & 2 & 2 & 0 & 0 & 4 \\
\hline $5 \times 4 \mathrm{DH}$ & W & 1 & 0 & 0 & 0 & 0 & 0 & 3 & 0 & 4 \\
\hline $5 \times 4 \mathrm{NDH}$ & W & 0 & 0 & 0 & 0 & 0 & 0 & 4 & 0 & 4 \\
\hline $4 \mathrm{x} 4 \mathrm{DH}$ & $\mathrm{W}$ & 1 & 0 & 0 & 0 & 1 & 0 & 3 & 0 & 5 \\
\hline $4 \mathrm{x} 4 \mathrm{NDH}$ & $\mathrm{W}$ & 1 & 0 & 0 & 0 & 0 & 1 & 2 & 0 & 4 \\
\hline Round DH & $\mathrm{W}$ & 0 & 0 & 0 & 0 & 0 & 2 & 2 & 0 & 4 \\
\hline Round NDH & W & 0 & 0 & 0 & 0 & 0 & 0 & 3 & 1 & 4 \\
\hline $5 \times 4 \mathrm{DH}$ & NW & 3 & 0 & 0 & 0 & 0 & 0 & 0 & 3 & 6 \\
\hline $5 \times 4 \mathrm{NDH}$ & NW & 5 & 0 & 0 & 0 & 0 & 0 & 0 & 4 & 9 \\
\hline $4 \times 4 \mathrm{DH}$ & NW & 8 & 0 & 0 & 0 & 0 & 0 & 0 & 3 & 11 \\
\hline $4 \times 4 \mathrm{NDH}$ & NW & 7 & 0 & 0 & 0 & 0 & 0 & 0 & 3 & 10 \\
\hline Round DH & NW & 2 & 0 & 0 & 1 & 0 & 1 & 1 & 1 & 6 \\
\hline Round NDH & NW & 1 & 0 & 0 & 0 & 0 & 1 & 1 & 2 & 5 \\
\hline
\end{tabular}




\section{A.2 Vibrating Motor Spec}

The vibrating motors came within the very first gloves assembled by the Department of Industrial Systems Engineering (ISE) at Virginia Tech. During the course of this research project we have ordered more motors due to the several glove models built and tested. All the purchases were from Jameco Electronics and the motors used in this project along with

other models can be found at www.jameco.com. Figure A.1 shows the specifications of the motor used in this research project. 


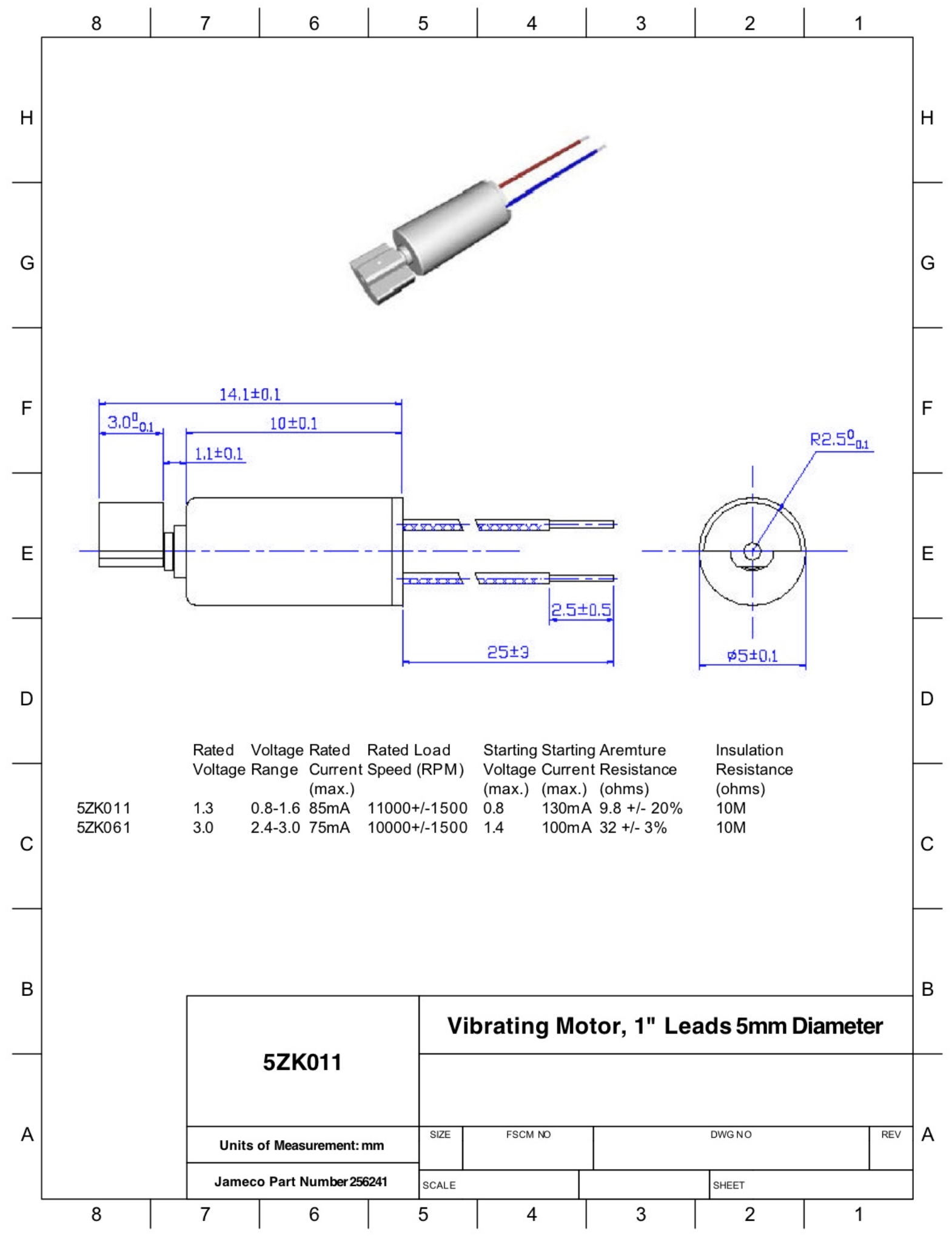

Figure A.1: Vibrating motor spefication sheet 


\section{A.3 Initial Studies Demographic Questionnaire}

Subject \#:

\section{Demographic Form}

Please complete the following questions.

Gender (circle one): $\quad$ Male Female

Age:

1. Occupation (if student, indicate graduate or undergraduate, and the year):

Major/Area of specialization (if student):

2. Tactile Displays are devices that convey information to the user through the sense of tact. Normally these devices are placed on contact with the skin or for the user to hold it. Have you ever used a system with this a tactile display? If so, please describe as follows:

(a) What type of display did you use?

(b) What kind of application was it (game, etc.)? 
(c) What kind of information was sent through the tactile display? 


\section{A.4 Initial Studies Post Questionnaire}

Please answer the following questions.

1. I felt comfortable wearing glove $\mathrm{A} / \mathrm{B} / \mathrm{C}$.

\begin{tabular}{|c|c|c|c|c|}
\hline $\begin{array}{l}\text { Strongly } \\
\text { agree }\end{array}$ & Disagree & $\begin{array}{l}\text { Neutral/No } \\
\text { opinion }\end{array}$ & Agree & Strongly Agree \\
\hline 1 & 2 & 3 & 4 & 5 \\
\hline
\end{tabular}

Comments:

2. I could perfectly feel the direction while wearing glove A/B/C.

\begin{tabular}{|c|c|c|c|c|}
\hline $\begin{array}{ll}\begin{array}{l}\text { Strongly } \\
\text { agree }\end{array} & \text { Dis- } \\
\end{array}$ & Disagree & $\begin{array}{l}\text { Neutral/No } \\
\text { opinion }\end{array}$ & Agree & Strongly Agree \\
\hline 1 & 2 & 3 & 4 & 5 \\
\hline
\end{tabular}

Comments: 
3. I perform better if I wear glove on my dominant hand.

\begin{tabular}{|c|c|c|c|c|}
\hline $\begin{array}{l}\text { Strongly Dis- } \\
\text { agree }\end{array}$ & Disagree & $\begin{array}{l}\text { Neutral/No } \\
\text { opinion }\end{array}$ & Agree & Strongly Agree \\
\hline 1 & 2 & 3 & 4 & 5 \\
\hline
\end{tabular}

Comments:

4. Wearing glove $\mathrm{A} / \mathrm{B} / \mathrm{C}$ did not interfere with my fingertip reading.

\begin{tabular}{|c|c|c|c|c|}
\hline $\begin{array}{ll}\text { Strongly } & \text { Dis- } \\
\text { agree } & \end{array}$ & Disagree & $\begin{array}{l}\text { Neutral/No } \\
\text { opinion }\end{array}$ & Agree & Strongly Agree \\
\hline 1 & 2 & 3 & 4 & 5 \\
\hline
\end{tabular}

Comments:

5. I could keep listening to what was being said while glove $\mathrm{A} / \mathrm{B} / \mathrm{C}$ was vibrating.

\begin{tabular}{|c|c|l|c|c|}
\hline $\begin{array}{l}\text { Strongly Dis- } \\
\text { agree }\end{array}$ & Disagree & $\begin{array}{l}\text { Neutral/No } \\
\text { opinion }\end{array}$ & Agree & Strongly Agree \\
\hline 1 & 2 & 3 & 4 & 5 \\
\hline
\end{tabular}

Comments: 
6. What suggestions would you give to improve our system? 



\section{Appendix B}

Chapter 3 - Additional Material 



\section{B.1 Catch Phrase Survey}

Non-Native Native Total \%Non-Native \%Native \%Non-Native+Native

1 Dont hold your breath

\begin{tabular}{lrrrrrr}
1 - Strongly disagree & 1 & 0 & 1 & $4.00 \%$ & $0.00 \%$ & $1.61 \%$ \\
2 - Disagree & 2 & 0 & 2 & $8.00 \%$ & $0.00 \%$ & $3.23 \%$ \\
3 - Neutral & 3 & 0 & 3 & $12.00 \%$ & $0.00 \%$ & $4.84 \%$ \\
4 - Agree & 11 & 15 & 26 & $44.00 \%$ & $40.54 \%$ & $41.94 \%$ \\
5 - Strongly Agree & 8 & 22 & 30 & $32.00 \%$ & $59.46 \%$ & $48.39 \%$ \\
\hline Total & 25 & 37 & 62 & &
\end{tabular}

2 Eat your heart out

1 - Strongly Disagree

2 - Disagree

3 - Neutral

4 - Agree

5 - Strongly agree

Total

\begin{tabular}{rr}
2 & 0 \\
8 & 2 \\
8 & 8 \\
6 & 18 \\
1 & 9 \\
\hline
\end{tabular}

\begin{tabular}{rrr}
$8.00 \%$ & $0.00 \%$ & $3.23 \%$ \\
$32.00 \%$ & $5.41 \%$ & $16.13 \%$ \\
$32.00 \%$ & $21.62 \%$ & $25.81 \%$ \\
$24.00 \%$ & $48.65 \%$ & $38.71 \%$ \\
$4.00 \%$ & $24.32 \%$ & $16.13 \%$ \\
\hline
\end{tabular}

\section{Egg your face}

1 - Strongly Disagree

2 - Disagree

3 - Neutral

4 - Agree

5 - Strongly agree

$\begin{array}{lll}25 & 37 & 62\end{array}$

\begin{tabular}{rr}
2 & $8.00 \%$ \\
10 & $32.00 \%$ \\
16 & $32.00 \%$ \\
24 & $24.00 \%$ \\
10 & $4.00 \%$ \\
\hline
\end{tabular}

$24.32 \%$

$16.13 \%$

Total

4 See you later, alligator

\begin{tabular}{lrrrrrr}
1 - Strongly Disagree & 1 & 1 & 2 & $4.00 \%$ & $2.70 \%$ & $3.23 \%$ \\
2 - Disagree & 6 & 4 & 10 & $24.00 \%$ & $10.81 \%$ & $16.13 \%$ \\
3 - Neutral & 4 & 2 & 6 & $16.00 \%$ & $5.41 \%$ & $9.68 \%$ \\
4 - Agree & 7 & 16 & 23 & $28.00 \%$ & $43.24 \%$ & $37.10 \%$ \\
5 - Strongly agree & 7 & 14 & 21 & $28.00 \%$ & $37.84 \%$ & $33.87 \%$ \\
\hline
\end{tabular}

Total

$\begin{array}{lll}25 & 37 & 62\end{array}$

5 Monkey see, monkey do

1 - Strongly Disagree
2 - Disagree
3 - Neutral
4 - Agree
5 - Strongly agree

Total

\begin{abstract}
My lips are sealed
\end{abstract}
1 - Strongly Disagree

2 - Disagree

3 - Neutral

4 - Agree

5 - Strongly agree

Total

\begin{tabular}{rrr}
5 & 0 \\
8 & 0 \\
4 & 4 & \\
2 & 19 & 21 \\
5 & 14 & 19 \\
\hline 24 & 37 & 61
\end{tabular}

5
8
8
21
19

$\begin{array}{r}20.83 \% \\ 33.33 \% \\ 16.67 \% \\ 8.33 \% \\ 20.83 \% \\ \hline\end{array}$

$0.00 \%$

$0.00 \%$

$10.81 \%$

$51.35 \%$

$37.84 \%$

$8.20 \%$

$13.11 \%$

$13.11 \%$

$34.43 \%$

$31.15 \%$
$41.94 \%$

$22.58 \%$

$.29 \%$ $3.23 \%$ 
$7 \quad$ Never a dull moment

\begin{tabular}{|c|c|c|c|c|c|c|}
\hline 1 - Strongly Disagree & 1 & 0 & 1 & $4.00 \%$ & $0.00 \%$ & $1.61 \%$ \\
\hline 2 - Disagree & 6 & 0 & 6 & $24.00 \%$ & $0.00 \%$ & $9.68 \%$ \\
\hline 3 - Neutral & 3 & 2 & 5 & $12.00 \%$ & $5.41 \%$ & $8.06 \%$ \\
\hline 4 - Agree & 5 & 18 & 23 & $20.00 \%$ & $48.65 \%$ & $37.10 \%$ \\
\hline 5 - Strongly agree & 10 & 17 & 27 & $40.00 \%$ & $45.95 \%$ & $43.55 \%$ \\
\hline Total & 25 & 37 & 62 & & & \\
\hline
\end{tabular}

\begin{tabular}{lrrrrrr} 
& & & & & \\
1 - Strongly Disagree & 3 & 0 & 3 & $12.00 \%$ & $0.00 \%$ & $4.84 \%$ \\
2 - Disagree & 4 & 2 & 6 & $16.00 \%$ & $5.41 \%$ & $9.68 \%$ \\
3 - Neutral & 6 & 3 & 9 & $24.00 \%$ & $8.11 \%$ & $14.52 \%$ \\
- Agree & 6 & 19 & 25 & $24.00 \%$ & $51.35 \%$ & $40.32 \%$ \\
5 - Strongly agre & 6 & 13 & 19 & $24.00 \%$ & $35.14 \%$ & $30.65 \%$ \\
\hline Total & 25 & 37 & 62 & &
\end{tabular}

9 Out of this world

\begin{tabular}{lrrrrrr}
1 - Strongly Disagree & 1 & 0 & 1 & $4.17 \%$ & $0.00 \%$ & $1.67 \%$ \\
2 - Disagree & 2 & 1 & 3 & $8.33 \%$ & $2.78 \%$ & $5.00 \%$ \\
3 - Neutral & 1 & 5 & 6 & $4.17 \%$ & $13.89 \%$ & $10.00 \%$ \\
4 - Agree & 8 & 12 & 20 & $33.33 \%$ & $33.33 \%$ & $33.33 \%$ \\
5 - Strongly agree & 12 & 18 & 30 & $50.00 \%$ & $50.00 \%$ & $50.00 \%$ \\
\hline Total & 24 & 36 & 60 &
\end{tabular}

10 The chips are down

\begin{tabular}{lrrrrrr} 
& & & & & \\
1 - Strongly Disagree & 2 & 4 & 6 & $8.00 \%$ & $10.81 \%$ & $9.68 \%$ \\
2 - Disagree & 9 & 6 & 15 & $36.00 \%$ & $16.22 \%$ & $24.19 \%$ \\
3 - Neutral & 4 & 13 & 17 & $16.00 \%$ & $35.14 \%$ & $27.42 \%$ \\
4 - Agree & 7 & 12 & 19 & $28.00 \%$ & $32.43 \%$ & $30.65 \%$ \\
5 - Strongly agree & 3 & 2 & 5 & $12.00 \%$ & $5.41 \%$ & $8.06 \%$ \\
\hline Total & 25 & 37 & 62 & &
\end{tabular}

\section{Vote early and vote often}

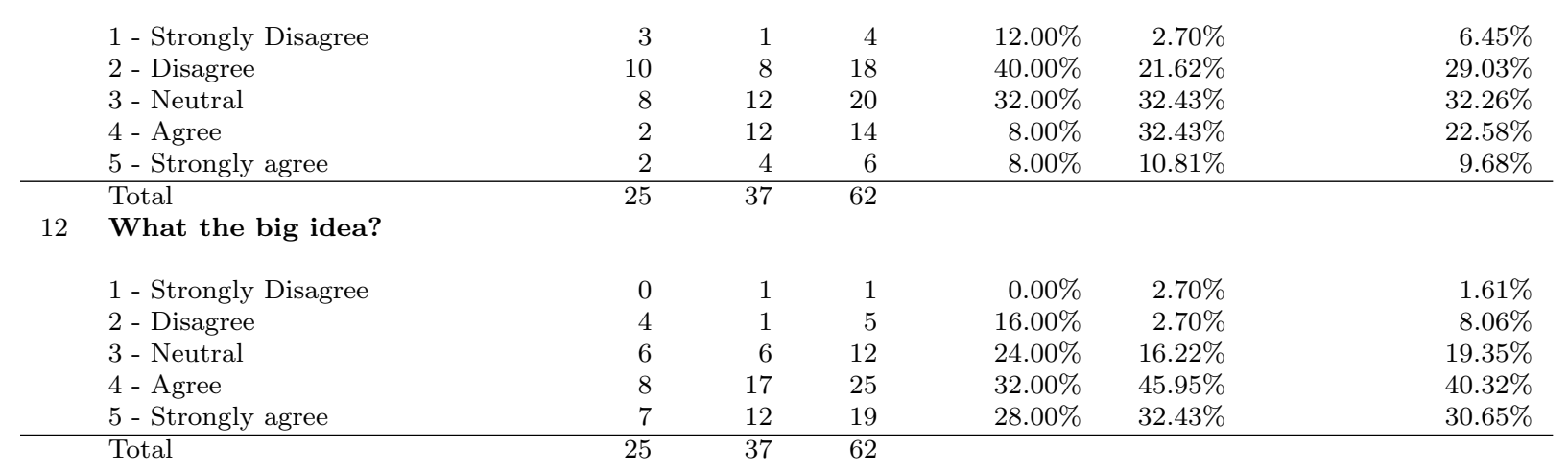




\section{B.2 Clue phrases questionnaire}

We are asking for your help to build a "clue-phrase" that would more easily lead to the catch phrase. One can choose any of the suggested words or build something entirely new, completely different.

\section{My lips are sealed}

Me / individual / Self / Myself / Own or other

Mouth / beak / mush / kisser or other

Be / exist / happen / comprised or other

Shut / closed / stamped / impermeable / secure or other

Completely different 4-word phrase:

Best pick so far:

\section{Me mouth is shut}

\section{Out of this world}

Outside / exterior / outside / exit or other

Off or other

globe / earth / terrene or other

Completely different 4-word phrase:

Best pick so far:

In the next galaxy

\section{Don't hold your breath}

No/not/veto/bar or other

Accommodate / stop / bar / take or other

thy _- or other

respiration / inhalation / insufflation or other

Completely different 4-word phrase:

Best pick so far:

No stop thy respiration

4. What's the big idea?

Which's / Whither's /Wherever's or other

a or other

huge / ample / jumbo / humungous or other

thought / estimation / concept / guess / theory or other

Completely different 4-word phrase:

Best pick so far:

What is going on 


\section{Never a dull moment}

Nevermore / ever / completely or other

the/one or other

ignorant / retarted / sluggish / dumb or other

instant / minute / bit or other

Completely different 4 -word phrase:

Best pick so far:

Nevermore one dumb moment

\section{See you later, alligator}

Gaze/ eye / catch / spy or other

Your or other

After/next / following / subsequent or other

Crocodile/reptile/lizard or other

Completely different 4 -word phrase:

Best pick so far:

\section{In a while crocodile}

\section{One for the road}

Uno / Only / Single or other

To / directed / towards / aimed or other

a or other

asphalt / roadway / pavement / route / path / way or other

Completely different 4-word phrase:

Best pick so far:

\section{Uno to a route}

\section{The chips are down}

parts / fragments or other

bellow / under / bottomward / depressed or other

Completely different 4 -word phrase:

Best pick so far:

The hands been played

9. Monkey see, monkey do

Ape/ primate / gorilla / simian or other

Eye / detect / perceive / observe / look or other

Ape / primate /gorilla /simian or other 
Make / execute / fulfill or other

Completely different 4 -word phrase:

Best pick so far:

\section{Gorilla look, gorilla execute}

10. Eat your heart out

Consume / devour / digest / dine / chew or other

thy or other

ventricule / auricle / pumper / puffer or other outside / outdoor / extinct /gone or other

Completely different 4 -word phrase:

Best pick so far:

Feast thy cardio outside

11. Vote early vote often

Select / choose/ take/ ballot or other

Immediate / quick / soon / promptly or other

Select / choose / take / ballot or other

Frequently / oftentimes / repeatedly or other

Completely different 4 -word phrase:

Best pick so far:

\section{Ballot soon ballot often}




\section{B.3 Blind follower post-questionnaire}

This post - questionnaire is supposed to be answered by the followers who are blind and used the system

1. I felt comfortable using the glove.

\begin{tabular}{|c|c|c|c|c|}
\hline $\begin{array}{l}\text { Strongly Dis- } \\
\text { agree }\end{array}$ & Disagree & $\begin{array}{l}\text { Neutral/No } \\
\text { opinion }\end{array}$ & Agree & Strongly Agree \\
\hline 1 & 2 & 3 & 4 & 5 \\
\hline
\end{tabular}

Comments:

2. I could perfectly feel the direction while using the glove.

\begin{tabular}{|c|c|c|c|c|}
\hline $\begin{array}{l}\text { Strongly } \\
\text { agree }\end{array}$ & Disagree & $\begin{array}{l}\text { Neutral/No } \\
\text { opinion }\end{array}$ & Agree & Strongly Agree \\
\hline 1 & 2 & 3 & 4 & 5 \\
\hline
\end{tabular}

Comments: 
3. I could perfectly listen to the guide while using the glove.

\begin{tabular}{|c|c|c|c|c|}
\hline $\begin{array}{l}\text { Strongly Dis- } \\
\text { agree }\end{array}$ & Disagree & $\begin{array}{l}\text { Neutral/No } \\
\text { opinion }\end{array}$ & Agree & Strongly Agree \\
\hline 1 & 2 & 3 & 4 & 5 \\
\hline
\end{tabular}

Comments:

4. The conversation between the guide and myself flowed naturally.

\begin{tabular}{|c|c|c|c|c|}
\hline $\begin{array}{ll}\text { Strongly } & \text { Dis- } \\
\text { agree } & \end{array}$ & Disagree & $\begin{array}{l}\text { Neutral/No } \\
\text { opinion }\end{array}$ & Agree & Strongly Agree \\
\hline 1 & 2 & 3 & 4 & 5 \\
\hline
\end{tabular}

Comments:

5. The system helped me get a better overview of the document where the letters were.

\begin{tabular}{|cc|c|c|c|c|}
\hline $\begin{array}{l}\text { Strongly } \\
\text { agree }\end{array}$ & Dis- & Disagree & $\begin{array}{l}\text { Neutral/No } \\
\text { opinion }\end{array}$ & Agree & Strongly Agree \\
\hline 1 & 2 & 3 & 4 & 5 \\
\hline
\end{tabular}

Comments: 
6. The constant change in directions in the glove made me disoriented

\begin{tabular}{|c|c|c|c|c|}
\hline $\begin{array}{l}\text { Strongly Dis- } \\
\text { agree }\end{array}$ & Disagree & $\begin{array}{l}\text { Neutral/No } \\
\text { opinion }\end{array}$ & Agree & Strongly Agree \\
\hline 1 & 2 & 3 & 4 & 5 \\
\hline
\end{tabular}

Comments:

7. I would perform better with practice

\begin{tabular}{|c|c|c|c|c|}
\hline $\begin{array}{ll}\text { Strongly } & \text { Dis- } \\
\text { agree } & \end{array}$ & Disagree & $\begin{array}{l}\text { Neutral/No } \\
\text { opinion }\end{array}$ & Agree & Strongly Agree \\
\hline 1 & 2 & 3 & 4 & 5 \\
\hline
\end{tabular}

Comments:

8. I would like to participate in future experiments because I believe this technology will help students who are blind.

\begin{tabular}{|c|c|c|c|c|}
\hline $\begin{array}{l}\text { Strongly } \\
\text { agree }\end{array}$ & Disagree & $\begin{array}{l}\text { Neutral/No } \\
\text { opinion }\end{array}$ & Agree & Strongly Agree \\
\hline 1 & 2 & 3 & 4 & 5 \\
\hline
\end{tabular}

Comments: 
9. The guide helped find where he/she was pointing at.

\begin{tabular}{|cc|c|c|c|c|}
\hline $\begin{array}{l}\text { Strongly } \\
\text { agree }\end{array}$ & Dis- & Disagree & $\begin{array}{l}\text { Neutral/No } \\
\text { opinion }\end{array}$ & Agree & Strongly Agree \\
\hline 1 & 2 & 3 & 4 & 5 \\
\hline
\end{tabular}

Comments:

10. I'd rather use this glove than have someone physically holding my hand and putting it over the document.

\begin{tabular}{|cc|c|c|c|c|}
\hline $\begin{array}{l}\text { Strongly } \\
\text { agree }\end{array}$ & Dis- & Disagree & $\begin{array}{l}\text { Neutral/No } \\
\text { opinion }\end{array}$ & Agree & Strongly Agree \\
\hline 1 & 2 & 3 & 4 & 5 \\
\hline
\end{tabular}

Comments:

11. If I had someone physically holding my hand and putting it over the document, instead of using the glove, I would have performed better.

\begin{tabular}{|cc|c|c|c|c|}
\hline $\begin{array}{l}\text { Strongly } \\
\text { agree }\end{array}$ & Dis- & Disagree & $\begin{array}{l}\text { Neutral/No } \\
\text { opinion }\end{array}$ & Agree & Strongly Agree \\
\hline 1 & 2 & 3 & 4 & 5 \\
\hline
\end{tabular}

Comments: 
12. Having access to where the tutor was pointing through the glove improved the conversation.

\begin{tabular}{|c|c|c|c|c|}
\hline $\begin{array}{l}\text { Strongly Dis- } \\
\text { agree }\end{array}$ & Disagree & $\begin{array}{l}\text { Neutral/No } \\
\text { opinion }\end{array}$ & Agree & Strongly Agree \\
\hline 1 & 2 & 3 & 4 & 5 \\
\hline
\end{tabular}

Comments:

13. I could read the letters while talking to the guide.

\begin{tabular}{|c|c|c|c|c|}
\hline $\begin{array}{ll}\text { Strongly } & \text { Dis- } \\
\text { agree } & \end{array}$ & Disagree & $\begin{array}{l}\text { Neutral/No } \\
\text { opinion }\end{array}$ & Agree & Strongly Agree \\
\hline 1 & 2 & 3 & 4 & 5 \\
\hline
\end{tabular}

Comments:

14. Using the system didn't interfere on my thinking of the solution.

\begin{tabular}{|cc|c|c|c|c|}
\hline $\begin{array}{l}\text { Strongly } \\
\text { agree }\end{array}$ & Dis- & Disagree & $\begin{array}{l}\text { Neutral/No } \\
\text { opinion }\end{array}$ & Agree & Strongly Agree \\
\hline 1 & 2 & 3 & 4 & 5 \\
\hline
\end{tabular}

Comments: 
15. Using the system made me pay more attention to the guide then I normally do in a regular class.

\begin{tabular}{|c|c|c|c|c|}
\hline $\begin{array}{l}\text { Strongly Dis- } \\
\text { agree }\end{array}$ & Disagree & $\begin{array}{l}\text { Neutral/No } \\
\text { opinion }\end{array}$ & Agree & Strongly Agree \\
\hline 1 & 2 & 3 & 4 & 5 \\
\hline
\end{tabular}

Comments:

16. Using the system made possible for me to access points visited previously, without the need of guide pointing again.

\begin{tabular}{|c|c|c|c|c|}
\hline $\begin{array}{l}\text { Strongly } \\
\text { agree }\end{array}$ & Disagree & $\begin{array}{l}\text { Neutral/No } \\
\text { opinion }\end{array}$ & Agree & Strongly Agree \\
\hline 1 & 2 & 3 & 4 & 5 \\
\hline
\end{tabular}

Comments:

17. I was able to point at my chart and ask questions.

\begin{tabular}{|c|c|c|c|c|}
\hline $\begin{array}{l}\text { Strongly Dis- } \\
\text { agree }\end{array}$ & Disagree & $\begin{array}{l}\text { Neutral/No } \\
\text { opinion }\end{array}$ & Agree & Strongly Agree \\
\hline 1 & 2 & 3 & 4 & 5 \\
\hline
\end{tabular}

Comments: 
18. I used pointing to help avoid misunderstanding in what I said.

\begin{tabular}{|c|c|c|c|c|}
\hline $\begin{array}{l}\text { Strongly Dis- } \\
\text { agree }\end{array}$ & Disagree & $\begin{array}{l}\text { Neutral/No } \\
\text { opinion }\end{array}$ & Agree & Strongly Agree \\
\hline 1 & 2 & 3 & 4 & 5 \\
\hline
\end{tabular}

Comments:

19. Due to pointing, I could speak less and be more precise in what I said.

\begin{tabular}{|c|c|c|c|c|}
\hline $\begin{array}{ll}\text { Strongly } & \text { Dis- } \\
\text { agree } & \end{array}$ & Disagree & $\begin{array}{l}\text { Neutral/No } \\
\text { opinion }\end{array}$ & Agree & Strongly Agree \\
\hline 1 & 2 & 3 & 4 & 5 \\
\hline
\end{tabular}

Comments:

20. Because of the system, I asked fewer clarification questions than I would normally ask.

\begin{tabular}{|cc|c|c|c|c|}
\hline $\begin{array}{l}\text { Strongly } \\
\text { agree }\end{array}$ & Dis- & Disagree & $\begin{array}{l}\text { Neutral/No } \\
\text { opinion }\end{array}$ & Agree & Strongly Agree \\
\hline 1 & 2 & 3 & 4 & 5 \\
\hline
\end{tabular}

Comments: 
21. The system gave me a better understanding of what the guide was trying to say.

\begin{tabular}{|c|c|c|c|c|}
\hline $\begin{array}{l}\text { Strongly Dis- } \\
\text { agree }\end{array}$ & Disagree & $\begin{array}{l}\text { Neutral/No } \\
\text { opinion }\end{array}$ & Agree & Strongly Agree \\
\hline 1 & 2 & 3 & 4 & 5 \\
\hline
\end{tabular}

Comments:

22. Because of the system, I believe that my intentions was better understood.

\begin{tabular}{|c|c|c|c|c|}
\hline $\begin{array}{l}\text { Strongly Dis- } \\
\text { agree }\end{array}$ & Disagree & $\begin{array}{l}\text { Neutral/No } \\
\text { opinion }\end{array}$ & Agree & Strongly Agree \\
\hline 1 & 2 & 3 & 4 & 5 \\
\hline
\end{tabular}

Comments:

23. What suggestions would you give to improve our system? 


\section{B.4 The answers the followers who are blind gave to their post-questionnaire}

Question

1. I felt comfortable using the glove.

$\begin{array}{llr}\text { Answer } & \text { Qty } & \text { Perct } \\ & & \\ \text { 1 - Strongly disagree } & 0 & 0.00 \% \\ \text { 2 - Disagree } & 0 & 0.00 \% \\ \text { 3 - Neutral / No opinion } & 0 & 0.00 \% \\ \text { 4 - Agree } & 3 & 75.00 \% \\ \text { 5 - Strongly Agree } & 1 & 25.00 \%\end{array}$

Comments. N: I think I could feel very comfortable with with practice.

2. I could perfectly feel the direction while using the glove.

$\begin{array}{llr}1 \text { - Strongly disagree } & 0 & 0.00 \% \\ 2 \text { - Disagree } & 1 & 25.00 \% \\ \text { 3 - Neutral / No opinion } & 0 & 0.00 \% \\ 4 \text { - Agree } & 1 & 25.00 \% \\ 5 \text { - Strongly Agree } & 2 & 50.00 \%\end{array}$

Comments. N: Heavy hand blocks the signal.

3. I could perfectly listen to the guide while using the glove

1 - Strongly disagree
2 - Disagree
3 - Neutral / No opinion
4 - Agree
5 - Strongly Agree

$0.00 \%$

$0.00 \%$

$25.00 \%$

$0.00 \%$

4. The conversation between the guide and myself flowed

5 - Strongly Agree

$100.00 \%$ naturally.

5. The system helped me get a better overview of the docu-

1 - Strongly disagree
2 - Disagree
3 - Neutral / No opinion
4 - Agree
5 - Strongly Agree

$0.00 \%$ $25.00 \%$

$0.00 \%$

$0.00 \%$

$75.00 \%$

1 - Strongly disagree
2 - Disagree
3 - Neutral / No opinion
4 - Agree
5 - Strongly Agree

$0.00 \%$

$0.00 \%$

$0.00 \%$

$50.00 \%$

$50.00 \%$

6. The constant change in directions in the glove made me disoriented.
$0.00 \%$ $25.00 \%$ $25.00 \%$ $25.00 \%$ $25.00 \%$

Comments. N: Frustrated because I wasn't able to find the right spot.

7. I performed better with practice.

$\begin{array}{llr}1 \text { - Strongly disagree } & 0 & 0.00 \% \\ \text { 2 - Disagree } & 0 & 0.00 \% \\ \text { 3 - Neutral / No opinion } & 0 & 0.00 \% \\ 4 \text { - Agree } & 0 & 0.00 \% \\ 5 \text { - Strongly Agree } & 4 & 100.00 \%\end{array}$


8. I would like to participate in future experiments because I believe this technology will help students who are blind.

9. The guide helped finding where he/she was pointing at.

1 - Strongly disagree
2 - Disagree
3 - Neutral / No opinion
4 - Agree
5 - Strongly Agree

$0.00 \%$

$0.00 \%$

$0.00 \%$

$0.00 \%$

5 - Strongly Agree

10. I'd rather use this glove than have someone physically

5 - Strongly Agree

$50.00 \%$ holding my hand and putting it over the document.

11. If I had someone physically holding my hand and putting

1 - Strongly disagree
2 - Disagree
3 - Neutral / No opinion
4 - Agree
5 - Strongly Agree

$0.00 \%$

$0.00 \%$

$50.00 \%$

$25.00 \%$

$25.00 \%$

it over the document, I would have performed better.

$\begin{array}{llr}1 \text { - Strongly disagree } & 0 & 0.00 \% \\ & & 75.00 \% \\ \text { 2 - Disagree } & 3 & 0.00 \% \\ \text { - Neutral / No opinion } & 0 & 0.00 \% \\ 4 \text { - Agree } & 0 & 25.00 \% \\ 5 \text { - Strongly Agree } & 1 & \end{array}$

Comments. R: As much as I don't like it, I agree.

12. Having access to where the tutor was point through the glove improved the conversation.

13. I could read the letters while talking to the guide.

1 - Strongly disagree
2 - Disagree
3 - Neutral / No opinion
4 - Agree
5 - Strongly Agree

1 - Strongly disagree
2 - Disagree
3 - Neutral / No opinion
4 - Agree
5 - Strongly Agree

$0.00 \%$

$0.00 \%$

$0.00 \%$

$50.00 \%$

$50.00 \%$

$0.00 \%$

$25.00 \%$

$0.00 \%$

$0.00 \%$

14. Using the system didn't interfere on my thinking of the

5 - Strongly Agree

$75.00 \%$ solution.

1 - Strongly disagree
2 - Disagree
3 - Neutral / No opinion
4 - Agree
5 - Strongly Agree

Comments. R: I strongly disagree because I had to concentrate on both. Besides, I was never good at charades when I was a kid. 
15. Using the system made me pay more attention to the guide than I normally do in regular class.

1 - Strongly disagree
2 - Disagree
3 - Neutral / No opinion
4 - Agree
5 - Strongly Agree

$0.00 \%$

$0.00 \%$

$0.00 \%$

$25.00 \%$

Comments. N: You get lost when they're at the board.

16. Using the system made possible for me to access point visited previously, without the need of the guide pointing again.

1 - Strongly disagree

1

$25.00 \%$

2 - Disagree

3 - Neutral / No opinion

4 - Agree

5 - Strongly Agree

$0.00 \%$

$75.00 \%$

$0.00 \%$

$0.00 \%$

17. I was able to point at my chart and ask questions.

1 - Strongly disagree
2 - Disagree
3 - Neutral / No opinion
4 - Agree
5 - Strongly Agree

$0.00 \%$

$0.00 \%$

$25.00 \%$

$25.00 \%$

$50.00 \%$

18. I used pointing to help avoid misunderstanding in what I said.

19.Due to pointing I could speak less and be more precise in

1 - Strongly disagree
2 - Disagree
3 - Neutral / No opinion
4 - Agree
5 - Strongly Agree

$0.00 \%$

$25.00 \%$

$50.00 \%$

$0.00 \%$

$25.00 \%$ what I said.

$\begin{array}{llr}1 \text { - Strongly disagree } & 0 & 0.00 \% \\ \text { 2 - Disagree } & 0 & 0.00 \% \\ \text { 3 - Neutral / No opinion } & 2 & 50.00 \% \\ 4 \text { - Agree } & 1 & 25.00 \% \\ 5 \text { - Strongly Agree } & 1 & 25.00 \%\end{array}$

Comments. N: I didn't point.

R: I strongly agree, but I'm a talkative person.

20. Because of the system, I asked fewer clarification questions than I would normally ask.

1 - Strongly disagree
2 - Disagree
3 - Neutral / No opinion
4 - Agree
5 - Strongly Agree

$0.00 \%$

$0.00 \%$

$25.00 \%$

$25.00 \%$

$50.00 \%$

21. The system gave me a better understanding of what the guide was trying to say.

$\begin{array}{llr}1 \text { - Strongly disagree } & 0 & 0.00 \% \\ \text { 2 - Disagree } & 0 & 0.00 \% \\ \text { 3 - Neutral / No opinion } & 0 & 0.00 \% \\ 4 \text { - Agree } & 2 & 50.00 \% \\ 5 \text { - Strongly Agree } & 2 & 50.00 \%\end{array}$


22. Because of the system, I perceived that my communication was better understood.

$\begin{array}{llr}1 \text { - Strongly disagree } & 0 & 0.00 \% \\ \text { 2 - Disagree } & 0 & 0.00 \% \\ \text { 3 - Neutral / No opinion } & 0 & 0.00 \% \\ 4 \text { - Agree } & 4 & 100.00 \% \\ 5 \text { - Strongly Agree } & 0 & 0.00 \%\end{array}$

Comments. N: She knew what I was saying.

23. What suggestions would you give to improve our system?

\begin{tabular}{|c|c|}
\hline Participant & Comment \\
\hline $\mathrm{M}$ & Too much vibration.. This system could be useful for actors and dancers. \\
\hline G & Widening of parameters for target zone. \\
\hline $\mathrm{R}$ & $\begin{array}{l}\text { Because it was tracking her (the guide) every move, I had problems figuring out where } \\
\text { she was pointing. Mavbe there is a way to filter out the tutor's pointing. }\end{array}$ \\
\hline & $\begin{array}{l}\text { I would suggest using regular Braille graph paper prepared with a manual Perkins unit. } \\
\text { Four motors instead of eight. }\end{array}$ \\
\hline
\end{tabular}




\section{B.5 Guide's post-questionnaire}

This post - questionnaire is supposed to be answered by the guides who played the game with the followers who are blind

1. I felt comfortable playing this game.

\begin{tabular}{|cc|c|l|c|c|}
\hline $\begin{array}{l}\text { Strongly } \\
\text { agree }\end{array}$ & Dis- & Disagree & $\begin{array}{l}\text { Neutral/No } \\
\text { opinion }\end{array}$ & Agree & Strongly Agree \\
\hline 1 & 2 & 3 & 4 & 5 \\
\hline
\end{tabular}

Comments:

2.The follower could easily access the letter I was pointig to.

\begin{tabular}{|cc|c|c|c|c|}
\hline $\begin{array}{l}\text { Strongly } \\
\text { agree }\end{array}$ & Dis- & Disagree & $\begin{array}{l}\text { Neutral/No } \\
\text { opinion }\end{array}$ & Agree & Strongly Agree \\
\hline 1 & 2 & 3 & 4 & 5 \\
\hline
\end{tabular}

Comments: 
3.The conversation flowed smoothly.

\begin{tabular}{|c|c|c|c|c|}
\hline $\begin{array}{l}\text { Strongly Dis- } \\
\text { agree }\end{array}$ & Disagree & $\begin{array}{l}\text { Neutral/No } \\
\text { opinion }\end{array}$ & Agree & Strongly Agree \\
\hline 1 & 2 & 3 & 4 & 5 \\
\hline
\end{tabular}

Comments:

4. I used pointing to help clarify myself and avoid misunderstanding.

\begin{tabular}{|c|c|c|c|c|}
\hline $\begin{array}{l}\text { Strongly Dis- } \\
\text { agree }\end{array}$ & Disagree & $\begin{array}{l}\text { Neutral/No } \\
\text { opinion }\end{array}$ & Agree & Strongly Agree \\
\hline 1 & 2 & 3 & 4 & 5 \\
\hline
\end{tabular}

Comments:

5. Because of the system, I could speak less and be more precise in what I said.

\begin{tabular}{|c|c|c|c|c|}
\hline $\begin{array}{l}\text { Strongly Dis- } \\
\text { agree }\end{array}$ & Disagree & $\begin{array}{l}\text { Neutral/No } \\
\text { opinion }\end{array}$ & Agree & Strongly Agree \\
\hline 1 & 2 & 3 & 4 & 5 \\
\hline
\end{tabular}

Comments:

6. I was able to understand the questions made by the follower when he/she was pointing at his/her puzzle.

\begin{tabular}{|c|c|c|c|c|}
\hline $\begin{array}{l}\text { Strongly Dis- } \\
\text { agree }\end{array}$ & Disagree & $\begin{array}{l}\text { Neutral/No } \\
\text { opinion }\end{array}$ & Agree & Strongly Agree \\
\hline 1 & 2 & 3 & 4 & 5 \\
\hline
\end{tabular}


Comments:

7. The follower's pointing was precise.

\begin{tabular}{|c|c|c|c|c|}
\hline $\begin{array}{l}\text { Strongly Dis- } \\
\text { agree }\end{array}$ & Disagree & $\begin{array}{l}\text { Neutral/No } \\
\text { opinion }\end{array}$ & Agree & Strongly Agree \\
\hline 1 & 2 & 3 & 4 & 5 \\
\hline
\end{tabular}

Comments:

8. The display helped me avoid misunderstanding on what the follower was saying.

\begin{tabular}{|c|c|c|c|c|}
\hline $\begin{array}{l}\text { Strongly Dis- } \\
\text { agree }\end{array}$ & Disagree & $\begin{array}{l}\text { Neutral/No } \\
\text { opinion }\end{array}$ & Agree & Strongly Agree \\
\hline 1 & 2 & 3 & 4 & 5 \\
\hline
\end{tabular}

Comments: 
9. The display helped me match my speech to the follower's pace.

\begin{tabular}{|c|c|c|c|c|}
\hline $\begin{array}{l}\text { Strongly Dis- } \\
\text { agree }\end{array}$ & Disagree & $\begin{array}{l}\text { Neutral/No } \\
\text { opinion }\end{array}$ & Agree & Strongly Agree \\
\hline 1 & 2 & 3 & 4 & 5 \\
\hline
\end{tabular}

Comments:

10. The display helped me getting a better understanding of the follower's behavior.

\begin{tabular}{|c|c|c|c|c|}
\hline $\begin{array}{ll}\text { Strongly } & \text { Dis- } \\
\text { agree } & \end{array}$ & Disagree & $\begin{array}{l}\text { Neutral/No } \\
\text { opinion }\end{array}$ & Agree & Strongly Agree \\
\hline 1 & 2 & 3 & 4 & 5 \\
\hline
\end{tabular}

Comments:

11. Having to look down at the display distracted me from giving instructions.

\begin{tabular}{|cc|c|c|c|c|}
\hline $\begin{array}{l}\text { Strongly } \\
\text { agree }\end{array}$ & Dis- & Disagree & $\begin{array}{l}\text { Neutral/No } \\
\text { opinion }\end{array}$ & Agree & Strongly Agree \\
\hline 1 & 2 & 3 & 4 & 5 \\
\hline
\end{tabular}

Comments: 
12. I was able to understand what the follower said while he/she was pointing at his/her chart.

\begin{tabular}{|cc|c|c|c|c|}
\hline $\begin{array}{l}\text { Strongly } \\
\text { agree }\end{array}$ & Dis- & Disagree & $\begin{array}{l}\text { Neutral/No } \\
\text { opinion }\end{array}$ & Agree & Strongly Agree \\
\hline 1 & 2 & 3 & 4 & 5 \\
\hline
\end{tabular}

Comments:

13. The follower showed less confusion than I expected.

\begin{tabular}{|c|c|c|c|c|}
\hline $\begin{array}{ll}\begin{array}{l}\text { Strongly } \\
\text { agree }\end{array} & \text { Dis- }\end{array}$ & Disagree & $\begin{array}{l}\text { Neutral/No } \\
\text { opinion }\end{array}$ & Agree & Strongly Agree \\
\hline 1 & 2 & 3 & 4 & 5 \\
\hline
\end{tabular}

Comments:

14. I believe the system will be useful in real world teaching.

\begin{tabular}{|c|c|c|c|c|}
\hline $\begin{array}{ll}\begin{array}{l}\text { Strongly } \\
\text { agree }\end{array} & \text { Dis- } \\
\end{array}$ & Disagree & $\begin{array}{l}\text { Neutral/No } \\
\text { opinion }\end{array}$ & Agree & Strongly Agree \\
\hline 1 & 2 & 3 & 4 & 5 \\
\hline
\end{tabular}

Comments: 
15. Because of the system, I asked less confirmation questions than I expected.

\begin{tabular}{|c|c|c|c|c|}
\hline $\begin{array}{l}\text { Strongly Dis- } \\
\text { agree }\end{array}$ & Disagree & $\begin{array}{l}\text { Neutral/No } \\
\text { opinion }\end{array}$ & Agree & Strongly Agree \\
\hline 1 & 2 & 3 & 4 & 5 \\
\hline
\end{tabular}

Comments:

16.Because of the system, I asked less clarification questions than I expected.

\begin{tabular}{|c|c|c|c|c|}
\hline $\begin{array}{ll}\text { Strongly } & \text { Dis- } \\
\text { agree } & \end{array}$ & Disagree & $\begin{array}{l}\text { Neutral/No } \\
\text { opinion }\end{array}$ & Agree & Strongly Agree \\
\hline 1 & 2 & 3 & 4 & 5 \\
\hline
\end{tabular}

Comments:

17. Because of the system, I percieved that my intentions were better understood.

\begin{tabular}{|cc|c|c|c|c|}
\hline $\begin{array}{l}\text { Strongly } \\
\text { agree }\end{array}$ & Dis- & Disagree & $\begin{array}{l}\text { Neutral/No } \\
\text { opinion }\end{array}$ & Agree & Strongly Agree \\
\hline 1 & 2 & 3 & 4 & 5 \\
\hline
\end{tabular}

Comments: 
18.The system helped me perceiving the follower's intentions.

\begin{tabular}{|c|c|c|c|c|}
\hline $\begin{array}{l}\text { Strongly Dis- } \\
\text { agree }\end{array}$ & Disagree & $\begin{array}{l}\text { Neutral/No } \\
\text { opinion }\end{array}$ & Agree & Strongly Agree \\
\hline 1 & 2 & 3 & 4 & 5 \\
\hline
\end{tabular}

Comments:

19. What suggestions would you give to improve our system? 


\section{B.6 The guides' answers to their postquestionnaire}

\section{Question}

1 I felt comfortable playing this game

The follower could easily access the letter I was pointig to

3 The conversation flowed smoothly

I used pointing to help clarify myself and avoid misundertanding

5 Because of the system, I could speak less and be more precise in what I said

6 I was able to understand the questions made by the follower when he/she was pointing at his/her puzzle

$7 \quad$ The follower's pointing was precise

8 The display helped me avoid misunderstanding on what the follower was saying

9 The display helped me match my speech to the follower's pace

10 The display helped me getting a better understanding of the follower's behavior
Pair Answer

$\mathrm{Da}+\mathrm{N}$

Do $+\mathrm{M}$

Do $+\mathrm{G}$

$\mathrm{Da}+\mathrm{N}$

$\mathrm{Do}+\mathrm{M}$

Do + G

$\mathrm{Da}+\mathrm{N}$

$\mathrm{Do}+\mathrm{M}$

Do + G

$\mathrm{Da}+\mathrm{N}$

$\mathrm{Do}+\mathrm{M}$

Do $+\mathrm{G}$

$\mathrm{Da}+\mathrm{N}$

$\mathrm{Do}+\mathrm{M}$

Do $+\mathrm{G}$

$\mathrm{Da}+\mathrm{N}$

$\mathrm{Do}+\mathrm{M}$

Do $+\mathrm{G}$

$\mathrm{Da}+\mathrm{N}$

Do $+\mathrm{M}$

Do $+\mathrm{G}$

$\mathrm{Da}+\mathrm{N}$

Do $+\mathrm{G}$

Do $+\mathrm{M}$

$\mathrm{Da}+\mathrm{N}$

$\mathrm{Do}+\mathrm{M}$

Do $+\mathrm{G}$

$\mathrm{Da}+\mathrm{N}$

$\mathrm{Do}+\mathrm{M}$

Do $+G$

5

5

5

4

4

5

5

3

5

5

5

5

\section{Guide's Comments}

Minus technical difficulty, she did well

He followed execently well
This system is self explanatory in use Cameras kept pace of all subjects moves 


\section{Question}

11 Having to look down at the display distracted me from giving instructions

12 I was able to understand what the follower said while he/she was pointing at his/her chart

13 The follower showed less confusion than I expected

$\begin{array}{ll}\mathrm{Da}+\mathrm{N} & 2 \\ & \\ \mathrm{Do}+\mathrm{M} & 3 \\ \mathrm{Do}+\mathrm{G} & 3\end{array}$

$\mathrm{Da}+\mathrm{N}$

$\mathrm{Do}+\mathrm{M}$

Do + G

$\mathrm{Da}+\mathrm{N}$

$\mathrm{Do}+\mathrm{M}$

Do + G

14 I believe the system will be useful in real world teaching

$\begin{array}{rl}\mathrm{Da}+\mathrm{N} & 5 \\ \mathrm{Do}+\mathrm{M} & 5 \\ \text { Do }+\mathrm{G} & 5\end{array}$

15 Because of the system, I asked less confirmation questions than I expected

$\mathrm{Da}+\mathrm{N}$

$\mathrm{Do}+\mathrm{M}$

Do + G

16 Because of the system, I asked less clarification questions than I expected

$\mathrm{Da}+\mathrm{N}$

$\mathrm{Do}+\mathrm{M}$

Do + G

I found I was still watching the display a lot. I believe with more practice (both student and teacher) would prove the display to be more second nature and less of a distraction

17 Because of the system, I percieved that my intentions were better understood

$\begin{array}{ll}\mathrm{Da}+\mathrm{N} & 5 \\ \mathrm{Do}+\mathrm{M} & 5\end{array}$

Do $+\mathrm{G} \quad 3$

18 The system helped me perceiving the follower's intentions

$\begin{array}{cc}\mathrm{Da}+\mathrm{N} & 5 \\ \mathrm{Do}+\mathrm{M} & 5 \\ \mathrm{Do}+\mathrm{G} & 4\end{array}$

19 What suggestions would you give to improve the system?

This session went very well and aside from some minor changes in the human glove interaction, the system worked well

Larger background display. Using overhead projector with transparencies

Larger display. Use of pointer, maybe 


\section{Appendix C}

\section{Chapter 4 - Additional Material}


Table C.1: Game effect on a deixis-laden discourse

\begin{tabular}{|c|c|c|c|c|c|c|}
\hline Part & Charade & Catch Phrase & Clue Phrase & $\begin{array}{l}\text { Time to } \\
\text { Complete } \\
\text { Charade }\end{array}$ & $\begin{array}{c}\text { Time to } \\
\text { Assemble } \\
\text { Clue Phrase }\end{array}$ & $\begin{array}{r}\text { Time to } \\
\text { map clue- } \\
\text { phrase to } \\
\text { Catch-Phrase }\end{array}$ \\
\hline \multirow[t]{9}{*}{$\mathbf{N}$} & \multirow{3}{*}{ 1st Chr } & Twinkle twinkle little star & Blink blink small sun & 323.50 & 229.50 & 93.00 \\
\hline & & My lips are sealed & Me mouth is shut & 314.85 & 216.99 & 97.86 \\
\hline & & Monkey see monkey do & Gorilla look gorilla execute & 336.14 & 312.14 & 23.99 \\
\hline & Avg 1st $\mathrm{Chr} b$ & \multirow{6}{*}{$\begin{array}{c}\text { see you later alligator } \\
\text { out of this world } \\
\text { one for the road }\end{array}$} & \multirow{6}{*}{$\begin{array}{l}\text { In a while crocodile } \\
\text { in the next galaxy } \\
\text { uno to a route }\end{array}$} & 324.83 & 252.88 & 71.62 \\
\hline & \multirow{3}{*}{ 2nd $\mathrm{Chr} \square^{c}$} & & & 198.00 & 128.00 & 70.00 \\
\hline & & & & 206.00 & 140.00 & 66.00 \\
\hline & & & & 295.00 & 177.00 & 118.00 \\
\hline & Avg 2nd Chr d & & & 233.00 & 148.33 & 84.67 \\
\hline & Gains & & & $28.27 \%$ & $41.34 \%$ & \\
\hline \multirow[t]{9}{*}{$\mathbf{R}$} & \multirow[t]{3}{*}{ 1st Chr } & Twinkle twinkle little star & Blink blink small sun & 605.00 & 605.00 & 0.00 \\
\hline & & My lips are sealed & Me mouth is shut & 433.00 & 342.00 & 91.00 \\
\hline & & Monkey see monkey do & Gorilla look gorilla execute & 626.00 & 611.00 & 15.00 \\
\hline & Avg 2nd Chr & & & 554.67 & 519.33 & 35.33 \\
\hline & \multirow[t]{3}{*}{ 2nd Chr } & out of this world & in the next galaxy & 466.00 & 191.00 & 275.00 \\
\hline & & see you later alligator & In a while crocodile & 153.00 & 144.00 & 9.00 \\
\hline & & Don't hold your breath & no stop thy respiration & 302.00 & 284.00 & 11.00 \\
\hline & Avg 2nd Chr & & & 307.00 & 206.33 & 98.33 \\
\hline & Gains & & & $44.65 \%$ & $60.27 \%$ & \\
\hline \multirow[t]{9}{*}{$\bar{G}$} & \multirow[t]{3}{*}{ 1st Chr } & Twinkle twinkle little star & Blink blink small sun & 548.30 & 544.30 & 4.00 \\
\hline & & Monkey see monkey do & Gorilla look gorilla execute & 396.00 & 391.00 & 5.00 \\
\hline & & My lips are sealed & Me mouth is shut & 412.50 & 350.00 & 62.50 \\
\hline & Avg 1st Chr & & & 452.27 & 428.43 & 23.83 \\
\hline & \multirow[t]{3}{*}{ 2nd Chr } & out of this world & in the next galaxy & 192.00 & 172.00 & 20.00 \\
\hline & & see you later alligator & In a while crocodile & 245.00 & 241.00 & 4.00 \\
\hline & & Don't hold your breath & no stop thy respiration & - & - & - \\
\hline & Avg 2nd Chr & & & 218.50 & 206.50 & 12.00 \\
\hline & Gains & & & $51.69 \%$ & $51.80 \%$ & \\
\hline \multirow[t]{4}{*}{$\mathbf{O}$} & \multirow[t]{3}{*}{ 2nd Chr } & see you later alligator & In a while crocodile & 127.00 & 108.00 & 19.00 \\
\hline & & out of this world & in the next galaxy & 214.00 & 101.00 & 113.00 \\
\hline & & one for the road & uno to a route & 261.00 & 106.00 & 155.00 \\
\hline & Avg 2nd Chr & & & 200.67 & 105.00 & 95.67 \\
\hline \multirow[t]{4}{*}{$\mathbf{A}$} & \multirow[t]{3}{*}{ 2nd Chr } & Don't hold your breath & no stop thy respiration & 406.00 & 346.00 & 60.00 \\
\hline & & See you later alligator & In a while crocodile & 359.00 & 350.00 & 9.00 \\
\hline & & One for the road & Uno to a route & 229.00 & 185.00 & 44.00 \\
\hline & Avg 2nd Chr & & & 331.33 & 293.67 & 37.67 \\
\hline
\end{tabular}

${ }^{a} 1$ st Chr - Before playing the game

${ }^{b}$ Avg 1st Chr - Averages before the game

${ }^{c}$ 2nd Chr - After playing the game

${ }^{d}$ Avg 2nd Chr - Averages after the game

${ }^{e}$ System malfunction 
Table C.2: Discourse level analysis

\begin{tabular}{|c|c|c|c|c|}
\hline Participant & Charade & Catch Phrase & Clue Phrase & $\begin{array}{r}\text { \% of object- } \\
\text { level } \\
\text { turns }\end{array}$ \\
\hline \multirow[t]{4}{*}{$\mathbf{N}$} & 1st Charade & $\begin{array}{c}\text { Twinkle twinkle little star } \\
\text { My lips are sealed } \\
\text { Monkey see monkey do }\end{array}$ & $\begin{array}{c}\text { Blink blink small sun } \\
\text { Me mouth is shut } \\
\text { Gorilla look gorilla execute }\end{array}$ & $\begin{array}{l}66.36 \% \\
68.83 \% \\
61.82 \%\end{array}$ \\
\hline & Avg 1st Charade & & & $65.67 \%$ \\
\hline & 2nd Charade & $\begin{array}{l}\text { see you later alligator } \\
\text { out of this world } \\
\text { one for the road }\end{array}$ & $\begin{array}{l}\text { In a while crocodile } \\
\text { in the next galaxy } \\
\text { uno to a route }\end{array}$ & $\begin{array}{r}97.26 \% \\
100.00 \% \\
100.00 \%\end{array}$ \\
\hline & Avg 2nd Charade & & & $99.09 \%$ \\
\hline \multirow[t]{4}{*}{$\overline{\mathbf{R}}$} & 1st Charade & $\begin{array}{c}\text { Twinkle twinkle little star } \\
\text { My lips are sealed } \\
\text { Monkey see monkey do }\end{array}$ & $\begin{array}{c}\text { Blink blink small sun } \\
\text { Me mouth is shut } \\
\text { Gorilla look gorilla execute }\end{array}$ & $\begin{array}{l}55.56 \% \\
64.61 \% \\
59.52 \%\end{array}$ \\
\hline & Avg 1st Charade & & & $59.90 \%$ \\
\hline & 2nd Charade & $\begin{array}{c}\text { out of this world } \\
\text { see you later alligator } \\
\text { Don't hold your breath }\end{array}$ & $\begin{array}{l}\text { in the next galaxy } \\
\text { In a while crocodile } \\
\text { no stop thy respiration }\end{array}$ & $\begin{array}{r}95.23 \% \\
93.87 \% \\
100.00 \%\end{array}$ \\
\hline & Avg 2nd Charade & & & $96.37 \%$ \\
\hline \multirow[t]{4}{*}{$\overline{\bar{G}}$} & 1st Charade & $\begin{array}{c}\text { Twinkle twinkle little star } \\
\text { Monkey see monkey do } \\
\text { My lips are sealed }\end{array}$ & $\begin{array}{c}\text { Blink blink small sun } \\
\text { Gorilla look gorilla execute } \\
\text { Me mouth is shut }\end{array}$ & $\begin{array}{l}42.33 \% \\
65.24 \% \\
65.17 \%\end{array}$ \\
\hline & Avg 1st Charade & & & $57.58 \%$ \\
\hline & 2nd Charade & $\begin{array}{c}\text { out of this world } \\
\text { see you later alligator } \\
\text { Don't hold your breath }\end{array}$ & $\begin{array}{l}\text { in the next galaxy } \\
\text { In a while crocodile } \\
\text { no stop thy respiration }\end{array}$ & $\begin{array}{r}93.87 \% \\
89.86 \% \\
-a^{a}\end{array}$ \\
\hline & Avg 2nd Charade & & & $91.87 \%$ \\
\hline \multirow[t]{2}{*}{$\overline{\mathrm{O}}$} & 2nd Charade & $\begin{array}{c}\text { see you later alligator } \\
\text { out of this world } \\
\text { one for the road }\end{array}$ & $\begin{array}{l}\text { In a while crocodile } \\
\text { in the next galaxy } \\
\text { uno to a route }\end{array}$ & $\begin{array}{r}100.00 \% \\
97.91 \% \\
97.82 \%\end{array}$ \\
\hline & Avg 2nd Charade & & & $98.58 \%$ \\
\hline \multirow[t]{2}{*}{$\overline{\mathbf{A}}$} & 2nd Charade & $\begin{array}{l}\text { Don't hold your breath } \\
\text { See you later alligator } \\
\text { One for the road }\end{array}$ & $\begin{array}{c}\text { no stop thy respiration } \\
\text { In a while crocodile } \\
\text { Uno to a route }\end{array}$ & $\begin{array}{r}97.59 \% \\
93.65 \% \\
100.00 \%\end{array}$ \\
\hline & Avg 2nd Charade & & & $97.08 \%$ \\
\hline
\end{tabular}

\footnotetext{
${ }^{a}$ System malfunction
} 
Table C.3: Comparing the blind follower's experience between the two charade studies

\begin{tabular}{|c|c|c|c|c|c|c|c|c|c|}
\hline Question & Charade & & & $\mathbf{P a}$ & icip & $\overline{a n}$ & & & Average \\
\hline & & $\mathbf{R}$ & G & $\mathbf{N}$ & $\mathbf{A}$ & & $\mathbf{O}^{a}$ & $\mathbf{M b}$ & \\
\hline I felt comfortable using the glove & 2 & 5 & 5 & 4 & & 3 & 5 & & 4.40 \\
\hline & 1 & 4 & 5 & 4 & & & & 4 & 4.25 \\
\hline I could perfectly feel the direction while using the glove & 2 & 5 & 2 & 5 & 4 & 4 & 5 & & 4.20 \\
\hline & 1 & 4 & 5 & 5 & & & & 2 & 4.00 \\
\hline I could perfectly listen to the guide while using the glove & 2 & 5 & 5 & 5 & 5 & 5 & 5 & & 5.00 \\
\hline & 1 & 5 & 5 & 5 & & & & 3 & 4.50 \\
\hline I'd rather use this glove than have someone physically move my & 2 & 5 & 5 & 5 & 3 & 3 & 5 & & 4.60 \\
\hline & 1 & 5 & 3 & 4 & & & & 3 & 3.75 \\
\hline If I had someone physically holding my hand and putting it over & 2 & 5 & 4 & 1 & 3 & 3 & 1 & & 2.80 \\
\hline & 1 & 5 & 3 & 4 & & & & 3 & 3.75 \\
\hline I would perform better with practice & 2 & 5 & 4 & 5 & 4 & 4 & 5 & & 4.60 \\
\hline & 1 & 5 & 5 & 5 & & & & 5 & 5.00 \\
\hline I would like to participate in future experiments because I be- & 2 & 5 & 5 & 5 & 4 & 4 & 5 & & 4.80 \\
\hline & 1 & 5 & 5 & 5 & & & & 5 & 5.00 \\
\hline The conversation between the guide and myself flowed naturally & 2 & 5 & 5 & 5 & 4 & 4 & 5 & & 4.80 \\
\hline & 1 & 5 & 5 & 5 & & & & 2 & 4.25 \\
\hline I needed more information than provided by the glove to find & 2 & 1 & 4 & 1 & & 3 & 1 & & 2.00 \\
\hline & 1 & 5 & 4 & 5 & & & & 5 & 4.00 \\
\hline I could perfectly listen/pay attention to the guide's speech while & 2 & 5 & 2 & 5 & 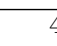 & 4 & 5 & & 4.20 \\
\hline & 1 & 5 & 5 & 5 & & & & 3 & 4.50 \\
\hline Having access to where the tutor was pointing through the glove & 2 & 5 & 5 & 5 & & 4 & 5 & & 4.80 \\
\hline & 1 & 5 & 5 & 4 & & & & 4 & 4.50 \\
\hline The system gave me a better understanding of what the guide & 2 & 5 & 5 & 3 & & 4 & 5 & & 4.40 \\
\hline & 1 & 4 & 5 & 5 & & & & 4 & 4.50 \\
\hline I was able to point at my chart and ask questions & 2 & 5 & 5 & 5 & & 3 & 5 & & 4.60 \\
\hline & 1 & 5 & 5 & 3 & & & & 4 & \\
\hline I used pointing to reduce misunderstanding in what I said & 2 & 5 & 5 & 5 & 2 & 2 & 3 & & 4.00 \\
\hline & 1 & 5 & 3 & 3 & & & & 2 & \\
\hline Because of the system, I perceived that my communication was & 2 & 4 & 5 & 5 & 3 & 3 & 5 & & 4.40 \\
\hline & 1 & 4 & 4 & 4 & & & & 4 & \\
\hline Using the system did not interfere on my thinking of the solution & 2 & 1 & 4 & 5 & & 4 & 5 & & 3.80 \\
\hline & 1 & 1 & 4 & 5 & & & & 5 & 3.20 \\
\hline
\end{tabular}

${ }^{a}$ Only second charade

${ }^{b}$ Only first charade 


\section{C.1 Game - Pre questionnaire}

Subject \#:

\section{Demographic Form}

Please complete the following questions.

Gender (circle one): $\quad$ Male $\quad$ Female

Age:

1. Occupation (if student, indicate graduate or undergraduate, and the year):

Major/Area of specialization (if student):

2. Tactile Displays are devices that convey information to the user through the sense of touch. Normally these devices are placed on contact with the skin or for the user to hold it. Have you ever used a computer tactile display? If so, please describe as follows:

(a) What type of display did you use?

(b) What kind of application was it (game, navigation systems, way finding devices, etc.)? 
(c) What kind of information was sent through the tactile display?

1. How often do you play computer games? What kind of games do you play?

\begin{tabular}{|l|l|l|l|l|}
\hline Never & Rarely & $\begin{array}{l}\text { At least once a } \\
\text { week }\end{array}$ & $\begin{array}{l}\text { At least twice a } \\
\text { week }\end{array}$ & $\begin{array}{l}\text { Every day or al- } \\
\text { most every day }\end{array}$ \\
\hline
\end{tabular}

Comments:

2. If you have played a game, tell us your experience... was it easy, hard? What kind of difficulties you encountered?

3. If you have played a computer game.. How long did it take before you felt that you were able to play effectively? Why? 
4. When you use a computer, do you use it with a keyboard, or do you use any special input device? Do you have a special keyboard? 


\section{C.2 Game - Post questionnaire}

Subject \#:

1. I played better with practice.

\begin{tabular}{|c|c|c|c|c|}
\hline $\begin{array}{ll}\begin{array}{l}\text { Strongly } \\
\text { agree }\end{array} & \text { Dis- }\end{array}$ & Disagree & $\begin{array}{l}\text { Neutral/No } \\
\text { opinion }\end{array}$ & Agree & Strongly Agree \\
\hline 1 & 2 & 3 & 4 & 5 \\
\hline
\end{tabular}

Comments:

2. I had to stay highly alert to get to the targets on time.

\begin{tabular}{|cc|c|c|c|c|}
\hline $\begin{array}{l}\text { Strongly } \\
\text { agree }\end{array}$ & Dis- & Disagree & $\begin{array}{l}\text { Neutral/No } \\
\text { opinion }\end{array}$ & Agree & Strongly Agree \\
\hline 1 & 2 & 3 & 4 & 5 \\
\hline
\end{tabular}

Comments: 
3. I had fun playing the game.

\begin{tabular}{|c|c|c|c|c|}
\hline $\begin{array}{l}\text { Strongly Dis- } \\
\text { agree }\end{array}$ & Disagree & $\begin{array}{l}\text { Neutral/No } \\
\text { opinion }\end{array}$ & Agree & Strongly Agree \\
\hline 1 & 2 & 3 & 4 & 5 \\
\hline
\end{tabular}

Comments:

4. I want to come back and finish the game.

\begin{tabular}{|c|c|c|c|c|}
\hline $\begin{array}{ll}\text { Strongly } & \text { Dis- } \\
\text { agree } & \end{array}$ & Disagree & $\begin{array}{l}\text { Neutral/No } \\
\text { opinion }\end{array}$ & Agree & Strongly Agree \\
\hline 1 & 2 & 3 & 4 & 5 \\
\hline
\end{tabular}

Comments:

5. The direction signals I received from the glove were timely and trustworthy.

\begin{tabular}{|cc|c|c|c|c|}
\hline $\begin{array}{l}\text { Strongly } \\
\text { agree }\end{array}$ & Dis- & Disagree & $\begin{array}{l}\text { Neutral/No } \\
\text { opinion }\end{array}$ & Agree & Strongly Agree \\
\hline 1 & 2 & 3 & 4 & 5 \\
\hline
\end{tabular}

Comments: 
6. I found level 1 challenging.

\begin{tabular}{|c|c|c|c|c|}
\hline $\begin{array}{l}\text { Strongly Dis- } \\
\text { agree }\end{array}$ & Disagree & $\begin{array}{l}\text { Neutral/No } \\
\text { opinion }\end{array}$ & Agree & Strongly Agree \\
\hline 1 & 2 & 3 & 4 & 5 \\
\hline
\end{tabular}

Comments:

7. I found level 2 challenging.

\begin{tabular}{|c|c|c|c|c|}
\hline $\begin{array}{ll}\text { Strongly } & \text { Dis- } \\
\text { agree } & \end{array}$ & Disagree & $\begin{array}{l}\text { Neutral/No } \\
\text { opinion }\end{array}$ & Agree & Strongly Agree \\
\hline 1 & 2 & 3 & 4 & 5 \\
\hline
\end{tabular}

Comments:

8. I found level 3 challenging.

\begin{tabular}{|c|c|c|c|c|}
\hline $\begin{array}{ll}\begin{array}{l}\text { Strongly } \\
\text { agree }\end{array} & \text { Dis- } \\
\end{array}$ & Disagree & $\begin{array}{l}\text { Neutral/No } \\
\text { opinion }\end{array}$ & Agree & Strongly Agree \\
\hline 1 & 2 & 3 & 4 & 5 \\
\hline
\end{tabular}

Comments: 
9. I found level 1 boring.

\begin{tabular}{|c|c|c|c|c|}
\hline $\begin{array}{l}\text { Strongly Dis- } \\
\text { agree }\end{array}$ & Disagree & $\begin{array}{l}\text { Neutral/No } \\
\text { opinion }\end{array}$ & Agree & Strongly Agree \\
\hline 1 & 2 & 3 & 4 & 5 \\
\hline
\end{tabular}

Comments:

10. I found level 2 boring.

\begin{tabular}{|c|c|c|c|c|}
\hline $\begin{array}{ll}\text { Strongly } & \text { Dis- } \\
\text { agree } & \end{array}$ & Disagree & $\begin{array}{l}\text { Neutral/No } \\
\text { opinion }\end{array}$ & Agree & Strongly Agree \\
\hline 1 & 2 & 3 & 4 & 5 \\
\hline
\end{tabular}

Comments:

11. I found level 3 boring.

\begin{tabular}{|cc|c|c|c|c|}
\hline $\begin{array}{l}\text { Strongly } \\
\text { agree }\end{array}$ & Dis- & Disagree & $\begin{array}{l}\text { Neutral/No } \\
\text { opinion }\end{array}$ & Agree & Strongly Agree \\
\hline 1 & 2 & 3 & 4 & 5 \\
\hline
\end{tabular}

Comments: 
12. I can do better if I could play game more.

\begin{tabular}{|c|c|c|c|c|}
\hline $\begin{array}{l}\text { Strongly Dis- } \\
\text { agree }\end{array}$ & Disagree & $\begin{array}{l}\text { Neutral/No } \\
\text { opinion }\end{array}$ & Agree & Strongly Agree \\
\hline 1 & 2 & 3 & 4 & 5 \\
\hline
\end{tabular}

Comments:

13. I was so absorbed by the game that I lost track of time.

\begin{tabular}{|c|c|c|c|c|}
\hline $\begin{array}{ll}\text { Strongly } & \text { Dis- } \\
\text { agree } & \end{array}$ & Disagree & $\begin{array}{l}\text { Neutral/No } \\
\text { opinion }\end{array}$ & Agree & Strongly Agree \\
\hline 1 & 2 & 3 & 4 & 5 \\
\hline
\end{tabular}

Comments:

14. I was so absorbed by the game that I that I forgot that I was wearing a glove, all I wanted to do was to find the targets.

\begin{tabular}{|c|c|l|c|c|}
\hline $\begin{array}{l}\text { Strongly Dis- } \\
\text { agree }\end{array}$ & Disagree & $\begin{array}{l}\text { Neutral/No } \\
\text { opinion }\end{array}$ & Agree & Strongly Agree \\
\hline 1 & 2 & 3 & 4 & 5 \\
\hline
\end{tabular}

Comments: 
15. I had full control of moving my character around to accomplish the mission.

\begin{tabular}{|c|c|c|c|c|}
\hline $\begin{array}{l}\text { Strongly Dis- } \\
\text { agree }\end{array}$ & Disagree & $\begin{array}{l}\text { Neutral/No } \\
\text { opinion }\end{array}$ & Agree & Strongly Agree \\
\hline 1 & 2 & 3 & 4 & 5 \\
\hline
\end{tabular}

Comments:

16. How much longer do you think you can play the game without feeling tired?

\begin{tabular}{|l|l|l|l|l|}
$5 \mathrm{~min}$ & $10 \mathrm{~min}$ & $15 \mathrm{~min}$ & $30 \mathrm{~min}$ & $45 \mathrm{~min}+$ \\
\hline
\end{tabular}

Comments:

17. How much longer do you think you can play the game without feeling bored?

\begin{tabular}{|c|c|c|c|c|}
\hline $5 \mathrm{~min}$ & $10 \mathrm{~min}$ & $15 \mathrm{~min}$ & $30 \mathrm{~min}$ & $45 \mathrm{~min}+$ \\
\hline
\end{tabular}

Comments: 
18. Any other suggestions? 



\section{Appendix D}

Chapter 5 - Additional Material 



\section{D.1 Curriculum A}

\section{D.1.1 First Lesson}

Title: Sun Rays

Lesson Number: 1 of 3

\section{Objectives:}

1. Overview of the Eratosthenes' problem

2. Learn how to measure the sun's angle by using shadow lengths

3. Determine the movement of light rays entering the Earth's atmosphere

(a) Specifically at the Tropic of Capricorn and at the Summer Solstice

\section{Rational:}

This lesson will provide the groundwork for finding the sun's angle at Alexandria.

\section{Instructional Procedure:}

Discussion of Eratosthenes' method of finding the radius of the Earth's radius

To measure the angle of the sun we can use a pole and the pole's shadow to calculate the angle of the sun. The following illustration demonstrates this.

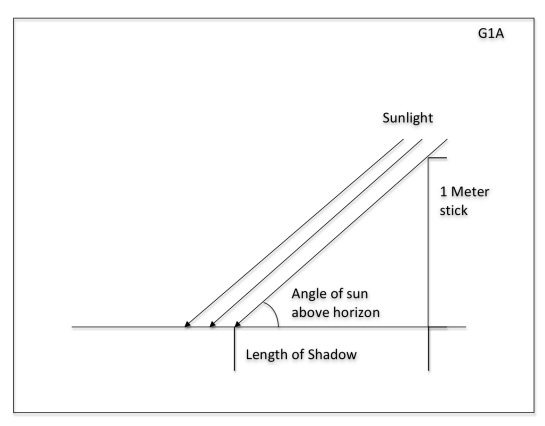

(a) Teacher's poster

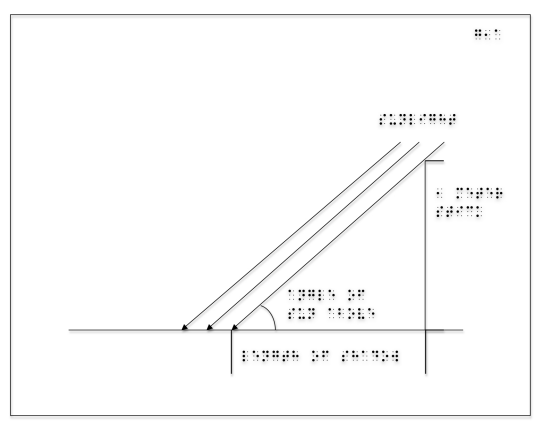

(b) Braille version

Figure D.1: Angle of the sun's rays

To get the sun's angle we use the trigonometric formula, Tan $\theta=$ Opposite/Adjacent.

This is possible because the pole (meter stick), the shadow, and the sunrays form a right triangle. 
For example: If we had a pole 6 feet high that we held perpendicular to the ground and measured the length of the shadow at 3.4 feet, we would find the angle of the sun by:

$\operatorname{Tan} \theta=6 / 3.4$

$\operatorname{Tan} \theta=1.764706$

$\theta=$ Inverse Tan (1.764706)

$\theta=60.46$ degrees

Another example, say you are able to measure the shadow of a light pole in your neighborhood. The shadow is 9.4 feet long and you know that the light pole is 7.5 feet tall. What is the angle of the sun?

Tan $\theta=7.5 / 9.4$

$\operatorname{Tan} \theta=.79787234$

$\theta=$ Inverse Tan $(.79787234)$

$\theta=38.58$ degrees

Eratosthenes made an assumption about sunrays entering the Earth's atmosphere. He assumed they were parallel. The diagram below illustrates this.

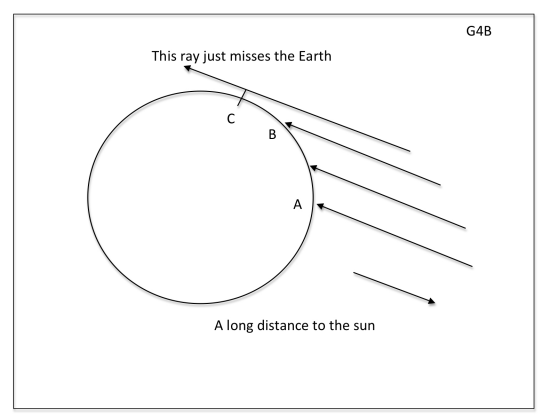

(a) Teacher's poster

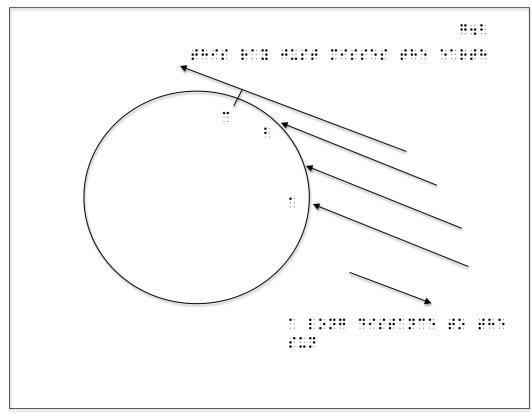

(b) Braille version

Figure D.2: Sun's rays assumed to be parallel

As it turns out Eratosthenes' assumption was okay to make, but only because of the large distance the sunrays travel before getting to Earth. This distance made so that the sunrays were very close to being parallel and could be assumed as being parallel. 


\section{D.1.2 Second Lesson}

\section{Lesson Title: Propositions and Proportions Lesson Number: 2 of 3}

\section{Objectives:}

1. To understand Euclid's 33 Proposition of Book IV.

2. To understand how to solve proportions when working with circles.

\section{Rational:}

This lesson will prepare the students for transferring the sun's angle measure to the center of the Earth and to allow them understanding on how to get the circumference of the Earth through solving proportional equations.

\section{Instructional Procedures:}

Euclid was a mathematician who lived in 300 B.C. and who thoroughly documented the field of geometry. His work is still in use today and is what is used as a basis for all of primary and high school geometry. That is, geometry in a plane (a flat surface, one with two dimensions) is called Euclidean Geometry. His documentation of this field is called Euclid's Elements and consists of 13 books which are filled with propositions, theorems, postulates, and definitions that are then "allowed" to be used when working with Euclidean geometry.

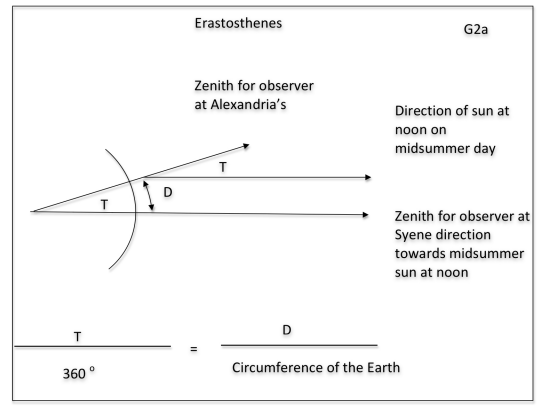

(a) Teacher's poster

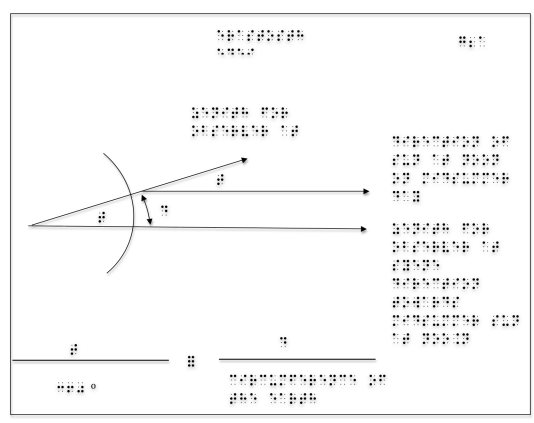

(b) Braille version

Figure D.3: Euclid's Proposition 33

Eratosthenes used one of Euclid's Propositions. It was Proposition 33 in book IV and it said "Angles in equal circles have the same ratio as the circumferences on which they stand whether they stand at the centers or at the circumference."

The diagram, above, shows this relationship. 
The second topic today is solving proportional equations.

The basic idea, here is that if you know the length of a piece of a circle's circumference and the center angle that makes that length, you can find the length of the circle's circumference.

Example:

Assume we know that segment $\mathrm{AB}$ is 12 inches long and that Angle ACB is 45 degrees.

We can find the length of the circumference by using a proportion. A proportional equation is one that shows a proportion for a part/whole relationship and equates that to another part/whole relationship. For this example, we have:

Length of $\frac{A B}{\text { Circumference }}=\frac{\text { Angle of } A C B}{360 \text { degrees }}$

$\frac{12 \text { inches }}{\text { Circumference }}=\frac{45}{360}$

Cross Multiply to get:

$45($ Circumference $)=12 \times 360$

$45($ Circumference $)=4320$

Circumference $=\frac{4320}{45}=96$ inches

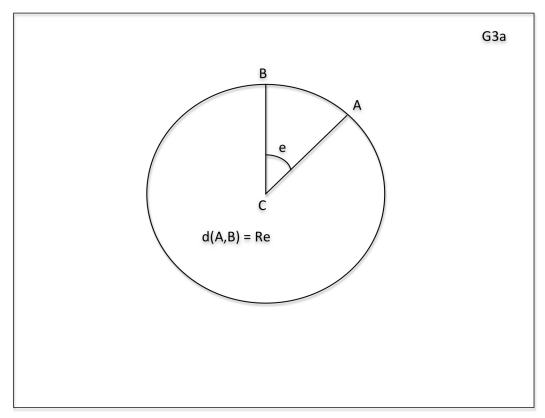

(a) Teacher's poster

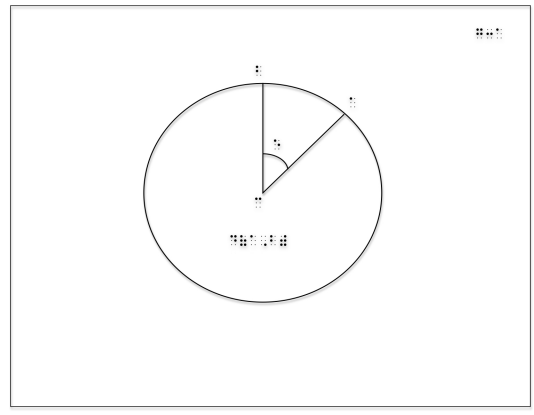

(b) Braille version

Figure D.4: Finding the length of the circle's circumference

Another example:

Say Angle ACB is 125 degrees and segment AB is 34 feet. What is the circumference of the circle?

The proportional equation will be:

$\frac{34 \text { feet }}{\text { Circumference }}=\frac{125}{360}$ degrees

$125($ Circumference $)=34 \times 360$

Circumference $=\frac{12,240}{125}=97.92$ feet 


\section{D.1.3 Third Lesson}

\section{Lesson Title: Circumference of the Earth \\ Lesson Number: 3 of 3}

\section{Objectives:}

1. To estimate the circumference of the Earth using the method of Eratosthenes.

\section{Instructional Procedures:}

Eratosthenes knew that the Earth was approximately a sphere. The cross section of the Earth could be seen, approximately, as a circle. Eratosthenes traveled between two cities which he believed were directly North/South of one another. These two cities were Alexandria and Syene. Syene is located on the Tropic of Cancer and Alexandria is 800 kilometers North of Syene.

First, Eratosthenes decided to take his measurements on the summer solstice, so that the angle of the sunrays at Syene would be 90 degrees (since it is located on the Tropic of Cancer). For Alexandria he found the angle of the sunrays by measuring shadows on the summer solstice (June 21) at noon.

Example:

Eratosthenes measured the angle of the sunrays with a Vertical pointer (thin stylus).

Say this pointer was 24 inches long and that the shadow was measured to be 3.03 inches.

What is the angle of the sunrays?

$\operatorname{Tan} \theta=\frac{24}{3.03}$

Tan $\theta=7.920792$

$\theta=$ Inverse Tan $(7.920792)$

$\theta=82.8045$

The difference between the angle of the sunrays in Alexandria and Syene is the angle measure Eratosthenes used to determine the circumference.

90 degrees -82.8045 degrees $=7.1955$ degrees

Round to 7.2 degrees

To find the circumference he used the following proportion:

$\frac{800 \mathrm{~km}}{\text { Circumference }}=\frac{7.2}{360}$ degrees

$7.2($ Circumference $)=800 \times 360$

$7.2($ Circumference $)=288,000$

Circumference $=40,000 \mathrm{~km}$ 


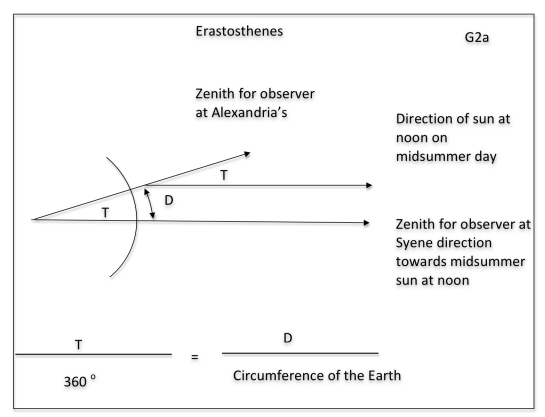

(a) Teacher's poster

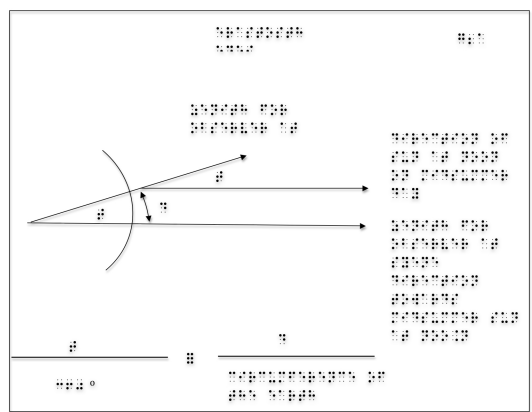

(b) Braille version

Figure D.5: Euclid's Proposition 33

Eratosthenes' predicted Circumference was between 39,690 and 46,620. The actual circumference of the Earth (from today's measures) is $40,075.16 \mathrm{~km}[1$.

Using this measure, we can determine the Radius and Diameter of the Earth.

Circumference $=2 \times \pi \times$ radius

$40,000=2 \times 3.14159 \times$ radius

$6366.20 \mathrm{~km}=$ radius

The diameter is twice the radius.

Diameter $=6,366.20 \times 2=12,732.40 \mathrm{~km}$ 


\section{D.2 Pre-test: Curriculum A}

1. Given the circumference of a circle, how would you find the circle's radius?

2. If we have a right triangle and we knew that the "opposite" side of an angle was 8 feet long and the "adjacent" side of that same angle was 20 feet long, what trigonometric function would we use to find the measure of the angle?

3. If you have a circle and you know that the diameter is 10 inches long, how would you find the length of the circumference?

4. How do you solve a proportional equation (one where a fraction equals another fraction)?

5. Where, approximately, are the Tropic of Capricorn and the Tropic of Cancer?

6. If we know the measurement of an angle in the center of a circle, how would we find the length of the line segment made from the angle?

7. How many degrees are in a circle?

8. What is the trigonometric function, Tangent equal to?

9. How could you measure the angle of the sun's rays?

10. If a small piece of a circle's circumference were marked, how could you find the length of the segment? 


\section{D.3 Post-test: Curriculum A}

1. Who was Eratosthenes? What is Eratosthenes known for?

2. Explain why it is important to assume that light rays from the Sun will strike the Earth in parallel rays? Why was it correct for Eratosthenes to assume that the sun rays would come to the Earth in parallel rays?

3. Given the circumference of a circle, how would you find the circles radius?

4. If the light pole was 8 feet high and the shadow was 20 feet long, how would we find the angle of the sun's rays?

5. Euclid's Proposition 33 in book IV says, "Angles in equal circles have the same ratio as the circumferences on which they stand whether they stand at the centers or at the circumference." What does this proposition mean? How did we use this Proposition to find the circumference?

6. If we know that a portion of a circle's circumference is 12 inches long and we know the center angle that makes this length to be 30 degrees, what do we use to find the length of the circumference?

7. Name three reasons why the circumference we arrived at for the Earth $(41,000 \mathrm{~km})$ was only an estimate.

8. What is special about the Tropics of Cancer and Capricorn? Why was it important that one of the cities used in Eratosthenes' experiment was located on one of these lines?

9. How do you solve a proportional equation?

10. What steps did Eratosthenes take to estimate the circumference of the earth? 


\section{D.4 Curriculum B}

\section{D.4.1 First lesson}

Title: Sine, Cosine and Tangent

Lesson Number: 1 of 3

\section{Objectives:}

1. To determine what sine, cosine, and tangent are and what they can be used to measure.

2. To practice problems that are solved using trigonometric functions.

\section{Rational:}

This lesson is meant as an introduction to trigonometry.

\section{Instructional Procedure:}

Trigonometric functions (also called circular functions) are functions of an angle. These functions are used to relate angles of a triangle to the lengths of the triangle's sides.

One main use of trigonometric functions is to define ratios of right triangle sides, based on the triangle's angles. To understand this use we need some basic information about right triangles.

A right triangle has one 90 degree angle. The side of the triangle, below, is based off of the given angle, A.

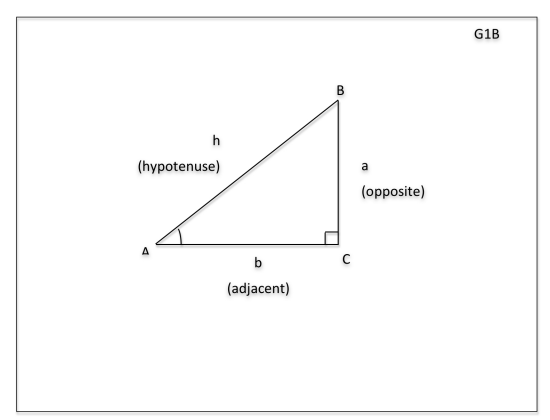

(a) Teacher's poster

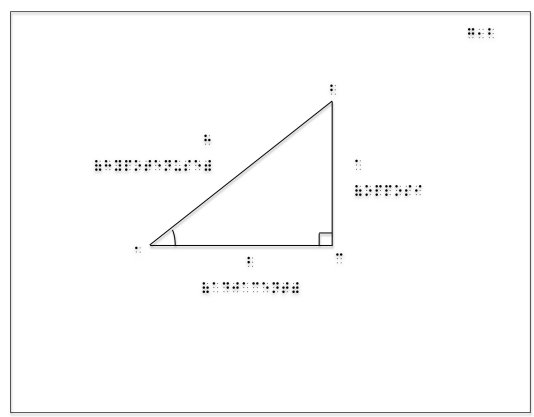

(b) Braille version

Figure D.6: Right Triangle

That is, the side across from Angle A is called opposite and the side next to Angle A is called adjacent. The longest side of a right triangle is called the hypotenuse. The hypotenuse is always located directly across from the right angle. If you wanted to find either the size of Angle A or the lengths of any of the three sides, you would use on of the Trigonometric functions, Sine, Cosine, or Tangent. The one you choose to use will depend on which information you need. 
Basic Trigonometric functions are:

Sine $(\mathrm{A})=\frac{\text { Opposite }}{\text { Hypothenuse }} ;$ Cosine $(\mathrm{A})=\frac{\text { Adjacent }}{\text { Hypothenuse }} ;$ Tangent $(\mathrm{A})=\frac{\text { Opposite }}{\text { Adjacent }}$

Examples: Given a right triangle that has an angle $\mathrm{A}=34$ degrees and a side length, $\mathrm{a}=14$ inches, find the length of the side adjacent to angle A.

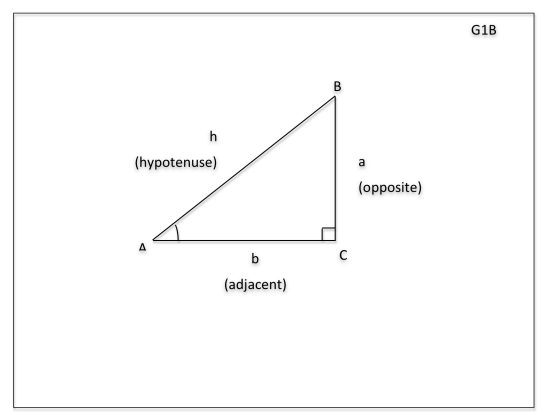

(a) Teacher's poster

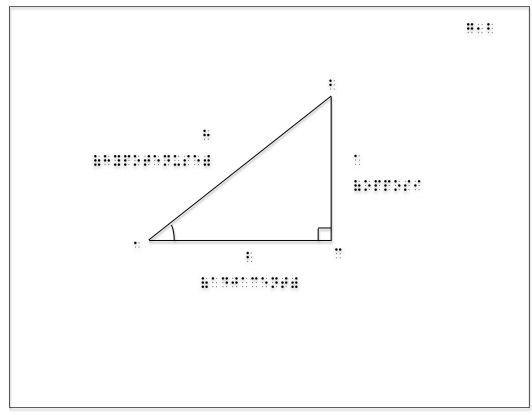

(b) Braille version

Figure D.7: Right Triangle - Example

Solution: The information given in the problem says that Angle $\mathrm{A}=34$ degrees and $\mathrm{a}=14$ inches. Since $a$ represents the Opposite Side (from angle A) and we are looking for the length of the adjacent side (from Angle A). Then we need to find a trigonometric function that uses these three variables.

Therefore, we will use Tangent.

$\tan (34)=\frac{14 \text { inches }}{\text { Adjacent }}$

To solve, we cross multiply.

$\tan (34) \times$ Hypothenuse $=14$ inches

Adjacent $=\frac{14 \text { inches }}{\tan (34)}$

Adjacent $=\frac{14 \text { inches }}{0.6745085168}$

Adjacent $=20.75585356$ inches (in our diagram this is $b$ )

For this triangle we now know the length of a and the length of b. We also know the angle measurments of Angle A and Angle C.

Next, we will find the angle measurement of Angle B.

To do this we need to first remember how many degrees are in a triangle.

This is 180 degrees.

Therefore, using the following equation we can figure out the measurement of Angle B.

$\operatorname{Angle}(A)+\operatorname{Angle}(B)+\operatorname{Angle}(C)=180$ degrees 
$34+\operatorname{Angle}(B)+90=180$

$124+\operatorname{Angle}(B)+=180$

$\operatorname{Angle}(B)=56$

We now know all three angle measures and two side lengths. Let us use another Trigonometric function to find the length of the hypotenuse.

If we use Angle A again, we can use side length b (the Adjacent Side) with the Cosine Function.

$\cos (A)=\frac{\text { Adjacent }}{\text { Hypothenuse }}$

$\cos (34)=\frac{20.7559}{\text { Hypothenuse }}$

Once again, we will use cross multiplication to solve.

$\cos (34) \times$ Hypothenuse $=20.7559$

Hypothenuse $=\frac{20.7559}{\cos (34)}$

Hypothenuse $=\frac{20.7559}{0.829036}$

Hypothenuse $=25.036$ degrees

Looking at the entire figure and its measurements, do you notice anything about the relationship between the angle measures and the lengths of the sides opposite those angles?

The larger the angle measure, the longer the opposite side will be. In this example, we have

Angle $\mathrm{A}=34$ degrees and the length of the opposite side is 14 inches

Angle $\mathrm{B}=56$ degrees and the length of the opposite side is 20.7559 inches

Angle $\mathrm{C}=90$ degrees and the length of the opposite side is 25.036 inches 


\section{D.4.2 Second Lesson}

Title: Sine and the Unit Circle

Lesson Number: 2 of 3

\section{Objective:}

1. To understand the relationship between using the Sine function and the unit circle.

\section{Instructional Procedure:}

We are interested in discovering the relationship between the unit circle and the Sine Curve. First we will look at some basic facts we know about the unit circle and the Sine Curve.

Assume the Unit Circle is placed on a $(\mathrm{x}, \mathrm{y})$ coordinate plane and that the $\mathrm{x}$-axis cuts it in half horizontally, and the $y$-axis cuts it in half vertically. Therefore the middle of the circle is placed at $(0,0)$. This divides the circle into four equal pieces. Each section will be 90 degrees. Each of these sections are called Quadrants. They are numbered in a counterclockwise manner. That is, the top right corner is called Quadrant I, the top left is Quadrant II, the next is Quadrant III, and the bottom right is Quadrant IV.

The Unit Circle has a radius of 1 unit. Therefore, in the diagram, the radius=hypotenuse $=1$ inch.

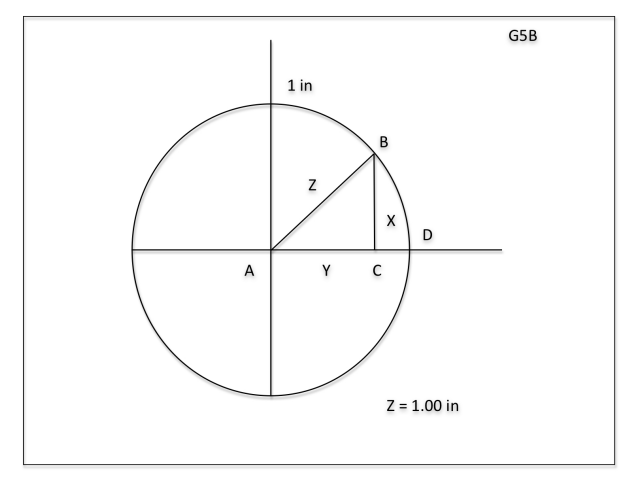

(a) Teacher's poster

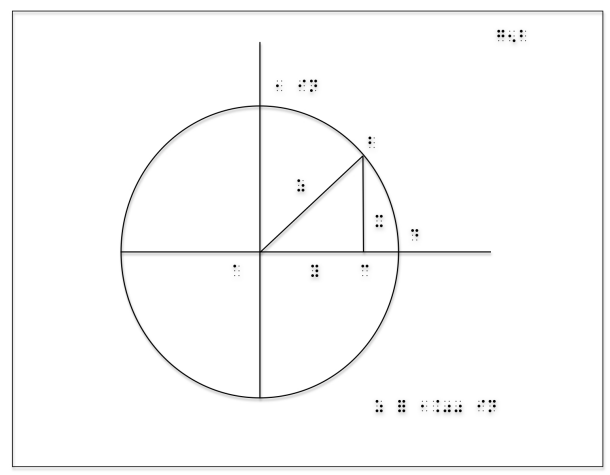

(b) Braille version

Figure D.8: First Graph

The figure shows a right triangle inside (inscribed) in the unit circle. We will use the Sine function to find the length of $x$. Assume that angle $\mathrm{A}$ is 45 degrees.

$\sin (A)=\frac{\text { Opposite }}{\text { Hypothenuse }}$

$\sin (45)=\frac{\text { Opposite }}{1 \text { inch }}$

Notice that the denominator is 1 . This will always be the case when we are using the unit circle (since the unit circle always has a radius of 1 unit). 
Therefore, when we cross multiply to solve for opposite, we get:

$\sin (45)=$ Opposite

$\sin (45)=0.7071067$ inch

This is what the sine answer means, it is the height of the opposite side from the given angle, when working with a unit circle.

Find the Sine of 30 degrees.

$\sin (30)=0.5$

This means that the height of the right triangle when the angle measure is 30 degrees, is half of an inch (or whatever unit you are using).

What happens if we have an angle measurement in Quadrant 4? (Look at the graph to see if anyone has a guess).

$\sin (330)=-0.5$

A negative means that the triangle is below the $\mathrm{x}$-axis.

What if we know the height of something and want to find the Sine angle that would make that height?

We would use the Inverse of Sine.

Assume our height is 0.5 inch.

$\sin (A)=0.5$

Inverse $\sin (0.5)=30$ degrees

Can anyone see what the Cosine might mean (based on our graph)? What is the formula for Cosine? (Cosine=Adjacent/Hypotenuse).

Therefore, Cosine is measuring the length of the horizontal leg of the triangle, $y$.

What is the length of b when Angle A is 45 ?

$\operatorname{cosin}(45)=0.70710678$

Does this number look familiar? It is the same length as the height of the triangle when Angle A was 45 .

To see why the height of the triangle is the same as the width of the triangle, we need to find out the angle measurements.

Angle A is 45 degrees

Angle $\mathrm{C}$ is 90 degrees

Angle B is what?

$45+90+$ Angle $\mathrm{B}=180$

Therefore Angle $\mathrm{B}=45$ degrees

So, Angle A = Angle B. This means that the lengths of the sides opposite those angles will be equal (just like last lesson when we learned that as the angle measure got bigger, so did the length of the opposite side of the triangle). 


\section{D.4.3 Third lesson}

Title: Angle Measurements: Radians and Degrees

Lesson Number: 3 of 3

\section{Objectives:}

1. To understand what $\pi$ means.

2. To understand the difference between a degree and a radian.

3. To be able to convert from degrees to radians and vis versa.

\section{Rational:}

When working with circles and with trigonometric functions $\pi$ is used regularly and most students only know $\pi$ as 3.14 and do not realize where that number comes from. Radians are defined in terms of pi and trigonometric functions are normally written in terms of radians.

\section{Instructional Procedure:}

For a circle, what is the circles diameter?

The length across a circle through its center point.

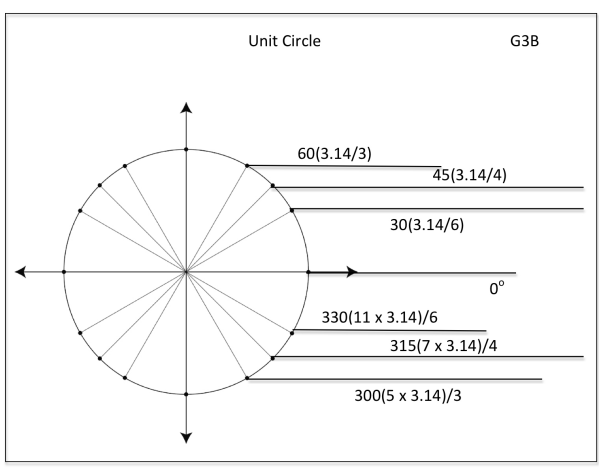

(a) Teacher's poster

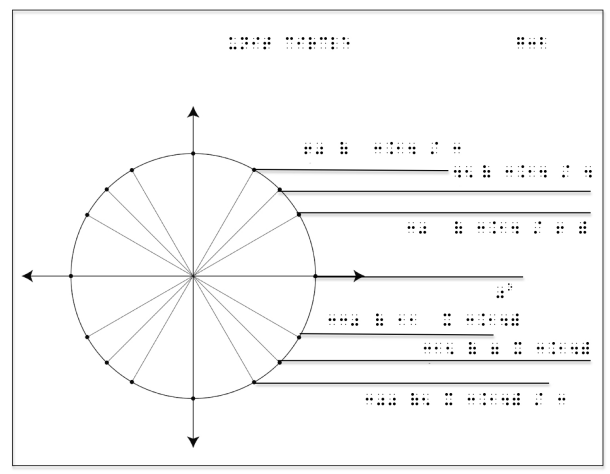

(b) Braille version

Figure D.9: Third Graph

If we take the diameter length of any circle and we see how many times we can fit that length around the outside of the circle (the circle's circumference) it will always be a little more than three diameters that will give you the circumference of the circle. In particular, it takes $3.14159 \ldots$ diameters to equal the circumference. This is $\pi$.

Therefore, $\pi=\frac{\text { Circumference }}{\text { Diameter }}$ 
This is where the common formula for finding the length of a circumference comes from. If we cross multiply we get:

Circumference $=\pi \times$ diameter

Some of you would have learned this formula as:

Circumference $=2 \times \pi \times$ Radius

Where did this come from? It is the same formula, except we are using the radius instead of the diameter. The radius is half of the diameter. Therefore, diameter $=2 \times$ radius.

Angle measurements are labeled in either "Degrees" or "Radians". We are going to figure out what a radian is and how we can switch from Radians to Degrees and vise versa.

You are probably more familiar with degrees. We know that a circle has 360 degrees, which mens that $\frac{1}{2}$ of a circle has $\frac{360}{2}=180$ degrees.

A radian is the angle at which the arc length formed by the angle is equal to the radius.

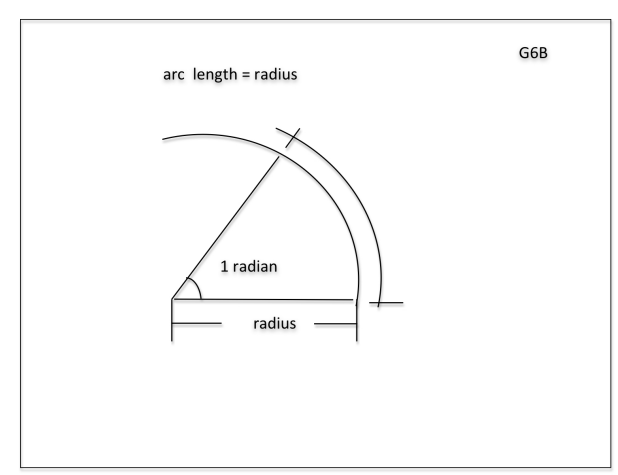

(a) Teacher's poster

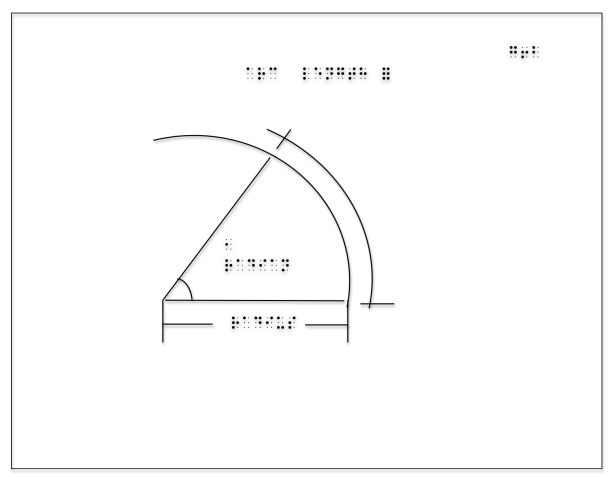

(b) Braille version

\section{Figure D.10: First Graph}

If you take the radius of any circle and you count how many times it will fit around half of the circle, you will get pi (a little more than three times). Do you see why?

We know that Circumference $=2 \times \pi \times$ radius and this tells us that,

Half of a circle is equal to radius $\times \pi$

$\frac{\text { Circumference }}{2}=$ radius $\times \pi$

We know that half of the circumference is 180 degrees.

This means 180 degrees $=$ radius $\times \pi$

radius $=\frac{180}{\pi}$ 
radian $=\frac{180 \text { degrees }}{\pi}=57.2958$ degrees

If we reverse this, we have, 1 degree $=\frac{\pi}{180 \times \text { radians }}$

Understanding some formulas become easier when working with Radians.

Let's look at how to change radians to degrees and vise versa.

We will use proportional reasoning (same as what we have been working on in our previous lessons).

How many degrees is the angle $\frac{\pi}{4}$ radians?

$\frac{\frac{\pi}{4}}{x \operatorname{deg}}=\frac{1 \text { radian }}{57.2958 \mathrm{deg}}$

Since we have a fraction that equals another fraction, we can use cross multiplication to find $\mathrm{x}$.

This gives us:

$\frac{\pi}{4} \times 57.2958=1 \mathrm{rad} \times x \mathrm{deg}$

$x=45 \mathrm{deg}$

Let us go the opposite direction and change a 30 degree angle measurement to radians. To do this we will leave the degrees as $\frac{180}{\pi}$ instead of using the estimate number of degrees. This way we will get an exact answer.

$\frac{30 \mathrm{deg}}{x \text { radians }}=\frac{\frac{180}{\pi}}{1 \text { radian }}$

Cross multiply to get:

$30 \operatorname{deg}(\operatorname{rad})=x \times \frac{180}{\pi}$

$\frac{30 \mathrm{deg}(1 \mathrm{rad})}{\frac{180}{\pi}}=x$

This bring us another concept in mathematics, which is how do we divide a complex fraction?

We leave the numerator (top number) as it is and we multiply that number by the reciprocal of the denominator.

$30 \times \frac{\pi}{180}=x$

This reduces to:

$x=\frac{\pi}{6}$ radians 


\section{D.5 Pre-test: Curriculum B}

1. What is the number $\pi$ ?

2. Given a right triangle where one of the legs is 10 inches long and the other leg is 15 inches long, how would you find the length of the hypotenuse?

3. What type of triangle is needed before you are able to use trigonometric (i.e. Sine, Cosine, Tangent) functions?

4. What is the trigonometric function of $\sin (\theta)$ ?

5. Besides degrees, what is another unit for measuring an angle?

6. If you know that a triangle has angle measurements of 42 degrees and 35 degrees, how would you find the measure of the final angle?

7. When a triangle is inside (or inscribed) in the unit circle, what does measure?

8. The $(\mathrm{x}, \mathrm{y})$ coordinate (think of the $\mathrm{x}$-axis and $\mathrm{y}$-axis crossing) is divided into four sections. What are these sections called?

9. What is the length of the radius in a unit circle?

10. When working with a right triangle inscribed in the unit circle, what is the hypotenuse of the triangle (in relation to the circle)? 


\section{D.6 Post-test: Curriculum B}

1. What type of triangle is needed in order to use one of the trigonometric functions (i.e. Sine, Cosine, Tangent)? What is the Tangent of an angle equal to?

2. Why is estimated as 3.14? How was this number found?

3. What is a radian? How did we find the measurement of 1 radian?

4. How would you change a radian measurement to a degree measurement?

5. When a right triangle is inscribed in a unit circle, what does the function Cosine measure?

6. How many degrees are in 1 radian? Can you give the exact number of degrees? What is the approximate number of degrees in 1 radian?

7. What angle measurements would cause the Sine of an angle to be negative?

8. If you know two angle measurements of a triangle, how would you find the measurement of the third angle?

9. When working with a right triangle inscribed in the unit circle, what is the hypotenuse of the triangle (in relation to the circle)?

10. Approximately how many radians would it take to equal the length of the circle's circumference? Explain your reasoning. 


\section{D.7 Student Demographic Questionnaire}

Please complete the following questions.

Student \#:

Gender (circle one): $\quad$ Male $\quad$ Female

Age :

Major:

Year:

1. Please list the math related college courses that you took (eg: Statistics, Computer Vision, etc)

2. Did you ever attend to an inclusive classroom (with sighted and blind) ? If so, please tell us about this experience. 


\section{D.8 Teacher Demographic Questionnaire}

Please complete the following questions.

Teacher \#:

Gender (circle one): $\quad$ Male $\quad$ Female

Age:

1. Occupation (if student, indicate graduate or undergraduate, and the year):

Major/Area of specialization (if student):

2. Please list the math related college courses that you took (eg: Statistics, Computer Vision, etc)

3. Please tell us about your experience with those who are blind. Did you ever work with them? Did you teach them? Did you attend any class with a student who is blind? For how long?

4. Are you a regular teacher/professor or a Teaching Assistant (TA)? If so, what class(es) do you teach/assist?

5. As a student, did you ever attend to an inclusive classroom (with sighted and blind) ? If so, please tell us about this experience. 
(20) 
This post - questionnaire is supposed to be answered by the INSTRUCTORS once they have finished lecturing all courses

\section{Please answer the following questions}

Teacher \#:

1. The instructional material was formatted properly.

\begin{tabular}{|c|c|c|c|c|}
\hline $\begin{array}{l}\text { Strongly Dis- } \\
\text { agree }\end{array}$ & Disagree & $\begin{array}{l}\text { Neutral/No } \\
\text { opinion }\end{array}$ & Agree & Strongly Agree \\
\hline 1 & 2 & 3 & 4 & 5 \\
\hline
\end{tabular}

Comments:

2. The students who are blind showed less confusion in classes with the augmentative system than in those without the system.

\begin{tabular}{|c|c|l|c|c|}
\hline $\begin{array}{l}\text { Strongly Dis- } \\
\text { agree }\end{array}$ & Disagree & $\begin{array}{l}\text { Neutral/No } \\
\text { opinion }\end{array}$ & Agree & Strongly Agree \\
\hline 1 & 2 & 3 & 4 & 5 \\
\hline
\end{tabular}

Comments: 
3. I felt more comfortable pointing at the charts and using expressions like "this, that" in classes with the augmentative system than in those without the system.

\begin{tabular}{|c|c|l|c|c|}
\hline $\begin{array}{l}\text { Strongly Dis- } \\
\text { agree }\end{array}$ & Disagree & $\begin{array}{l}\text { Neutral/No } \\
\text { opinion }\end{array}$ & Agree & Strongly Agree \\
\hline 1 & 2 & 3 & 4 & 5 \\
\hline
\end{tabular}

Comments:

4. I was able to express myself more effectively in classes with the augmentative system than in those without the system.

\begin{tabular}{|c|c|l|c|c|}
\hline $\begin{array}{l}\text { Strongly Dis- } \\
\text { agree }\end{array}$ & Disagree & $\begin{array}{l}\text { Neutral/No } \\
\text { opinion }\end{array}$ & Agree & Strongly Agree \\
\hline 1 & 2 & 3 & 4 & 5 \\
\hline
\end{tabular}

\section{Comments:}

5. The student who is blind's pointing was more precise in classes with the augmentative system than in those without the system.

\begin{tabular}{|c|c|l|c|c|}
\hline $\begin{array}{l}\text { Strongly Dis- } \\
\text { agree }\end{array}$ & Disagree & $\begin{array}{l}\text { Neutral/No } \\
\text { opinion }\end{array}$ & Agree & Strongly Agree \\
\hline 1 & 2 & 3 & 4 & 5 \\
\hline
\end{tabular}

\section{Comments:}


6. The display helped me avoid misunderstanding the student who is blind.

\begin{tabular}{|c|c|c|c|c|}
\hline $\begin{array}{ll}\text { Strongly } & \text { Dis- } \\
\text { agree } & \end{array}$ & Disagree & $\begin{array}{l}\text { Neutral/No } \\
\text { opinion }\end{array}$ & Agree & Strongly Agree \\
\hline 1 & 2 & 3 & 4 & 5 \\
\hline
\end{tabular}

Comments:

7. The display helped me match my speech to the student who is blind's pace.

\begin{tabular}{|c|c|c|c|c|}
\hline $\begin{array}{l}\text { Strongly } \\
\text { agree }\end{array}$ & Disagree & $\begin{array}{l}\text { Neutral/No } \\
\text { opinion }\end{array}$ & Agree & Strongly Agree \\
\hline 1 & 2 & 3 & 4 & 5 \\
\hline
\end{tabular}

Comments:

8. Having to look down at the display distracted me from lecturing.

\begin{tabular}{|c|c|l|c|c|}
\hline $\begin{array}{l}\text { Strongly Dis- } \\
\text { agree }\end{array}$ & Disagree & $\begin{array}{l}\text { Neutral/No } \\
\text { opinion }\end{array}$ & Agree & Strongly Agree \\
\hline 1 & 2 & 3 & 4 & 5 \\
\hline
\end{tabular}

Comments:

9. I was able to better understand what the student who is blind said while he/she was pointing at his/her chart and asking questions in classes with the augmentative system than in those without the system. 


\begin{tabular}{|c|c|l|c|c|}
\hline $\begin{array}{l}\text { Strongly Dis- } \\
\text { agree }\end{array}$ & Disagree & $\begin{array}{l}\text { Neutral/No } \\
\text { opinion }\end{array}$ & Agree & Strongly Agree \\
\hline 1 & 2 & 3 & 4 & 5 \\
\hline
\end{tabular}

Comments:

10. I believe the system will be useful in real world teaching.

\begin{tabular}{|c|c|l|c|c|}
\hline $\begin{array}{l}\text { Strongly Dis- } \\
\text { agree }\end{array}$ & Disagree & $\begin{array}{l}\text { Neutral/No } \\
\text { opinion }\end{array}$ & Agree & Strongly Agree \\
\hline 1 & 2 & 3 & 4 & 5 \\
\hline
\end{tabular}

Comments:

11. I feel that students understood my lecture better in classes with the augmentative system than in those without the system.

\begin{tabular}{|c|c|c|c|c|}
\hline $\begin{array}{l}\text { Strongly Dis- } \\
\text { agree }\end{array}$ & Disagree & $\begin{array}{l}\text { Neutral/No } \\
\text { opinion }\end{array}$ & Agree & Strongly Agree \\
\hline 1 & 2 & 3 & 4 & 5 \\
\hline
\end{tabular}

Comments:

12. I could understand student questions better in classes with the augmentative system than in those without the system. 


\begin{tabular}{|c|c|l|c|c|}
\hline $\begin{array}{l}\text { Strongly Dis- } \\
\text { agree }\end{array}$ & Disagree & $\begin{array}{l}\text { Neutral/No } \\
\text { opinion }\end{array}$ & Agree & Strongly Agree \\
\hline 1 & 2 & 3 & 4 & 5 \\
\hline
\end{tabular}

Comments:

13. I felt that the system impeded instead of helping the conversation. Please explain your answer.

\begin{tabular}{|c|c|c|c|c|}
\hline $\begin{array}{ll}\begin{array}{l}\text { Strongly } \\
\text { agree }\end{array} & \text { Dis- }\end{array}$ & Disagree & $\begin{array}{l}\text { Neutral/No } \\
\text { opinion }\end{array}$ & Agree & Strongly Agree \\
\hline 1 & 2 & 3 & 4 & 5 \\
\hline
\end{tabular}

Explain: 
14. Do you think the system slowed down the lecture or sped it up? You can assign a number ranging from 1 to 5 . 1 being that you think that the system definitely slowed down the lecture, 2 that the system somewhat slowed it down, 3 that you don't think it changed the lecturing speed, 4 meaning that it somewhat sped it up, and 5 that it definitely sped it up.

\begin{tabular}{|c|c|c|c|c|}
\hline $\begin{array}{l}\text { Definitely } \\
\text { slowed down }\end{array}$ & $\begin{array}{l}\text { Somewhat } \\
\text { slowed down }\end{array}$ & $\begin{array}{l}\text { Didn't change } \\
\text { the lecture } \\
\text { speed }\end{array}$ & $\begin{array}{l}\text { Somewhat sped } \\
\text { up }\end{array}$ & $\begin{array}{l}\text { Definitely sped } \\
\text { up }\end{array}$ \\
\hline 1 & 2 & 3 & 4 & 5 \\
\hline
\end{tabular}

Comments:

15. Please rate your preference between both teaching alternatives. You can assign a number ranging from 1 to 5.1 being that you definitely prefer lecturing inclusive classes in the traditional way, 2 that you are more inclined to the traditional way, 3 that you are indifferent, 4 that you are more inclined for classes with the augmentative system, 5 that you definitely prefer attending classes with the augmentative system. Please justify your answer.

\begin{tabular}{|c|l|l|l|l|}
\hline $\begin{array}{l}\text { Definitely no } \\
\text { augmentative } \\
\text { technology }\end{array}$ & $\begin{array}{l}\text { Somewhat in- } \\
\text { clined to no } \\
\text { augmentative } \\
\text { technology }\end{array}$ & $\begin{array}{l}\text { Somewhat } \\
\text { inclined to } \\
\text { augmentative } \\
\text { technology }\end{array}$ & $\begin{array}{l}\text { Definitely } \\
\text { inclined to } \\
\text { augmentative } \\
\text { technology }\end{array}$ \\
\hline 1 & 2 & 3 & 4 & 5 \\
\hline
\end{tabular}

Justification: 
16. I think students who are blind and attend the mini-courses with the help of the system will perform better on the post-test.

\begin{tabular}{|c|c|l|c|c|}
\hline $\begin{array}{l}\text { Strongly Dis- } \\
\text { agree }\end{array}$ & Disagree & $\begin{array}{l}\text { Neutral/No } \\
\text { opinion }\end{array}$ & Agree & Strongly Agree \\
\hline 1 & 2 & 3 & 4 & 5 \\
\hline
\end{tabular}

Explain:

17. Students who are blind were more engaged in classroom discussions in classes with the augmentative system than in those without the system.

\begin{tabular}{|c|c|c|c|c|}
\hline $\begin{array}{l}\text { Strongly } \\
\text { agree }\end{array}$ & Disagree & $\begin{array}{l}\text { Neutral/No } \\
\text { opinion }\end{array}$ & Agree & Strongly Agree \\
\hline 1 & 2 & 3 & 4 & 5 \\
\hline
\end{tabular}

Comments:

18. If this system is used in regular classrooms, the use of the cameras for tracking teacher and students who are blind would intimidate me.

\begin{tabular}{|c|c|l|c|c|}
\hline $\begin{array}{l}\text { Strongly Dis- } \\
\text { agree }\end{array}$ & Disagree & $\begin{array}{l}\text { Neutral/No } \\
\text { opinion }\end{array}$ & Agree & Strongly Agree \\
\hline 1 & 2 & 3 & 4 & 5 \\
\hline
\end{tabular}

Comments: 
19. The system disrupted the flow of the lecture more than having students who are blind without the system.

\begin{tabular}{|c|c|l|c|c|}
\hline $\begin{array}{l}\text { Strongly Dis- } \\
\text { agree }\end{array}$ & Disagree & $\begin{array}{l}\text { Neutral/No } \\
\text { opinion }\end{array}$ & Agree & Strongly Agree \\
\hline 1 & 2 & 3 & 4 & 5 \\
\hline
\end{tabular}

Comments:

20. Please give us suggestions to improve our system. 
This post - questionnaire is supposed to be answered by the STUDENTS WHO ARE BLIND who just attended to a course where the augmentative technology was employed and took the post-test Please answer the following questions

Student who is blind \#:

1. The course flowed as any other regular course.

\begin{tabular}{|c|c|c|c|c|}
\hline $\begin{array}{ll}\begin{array}{l}\text { Strongly } \\
\text { agree }\end{array} & \text { Dis- } \\
\text { agre }\end{array}$ & Disagree & $\begin{array}{l}\text { Neutral/No } \\
\text { opinion }\end{array}$ & Agree & Strongly Agree \\
\hline 1 & 2 & 3 & 4 & 5 \\
\hline
\end{tabular}

Comments:

2. Having access to the instructional material before class helped me in keeping up with the lecture.

\begin{tabular}{|c|c|c|c|c|}
\hline $\begin{array}{l}\text { Strongly } \\
\text { agree }\end{array}$ & Disagree & $\begin{array}{l}\text { Neutral/No } \\
\text { opinion }\end{array}$ & Agree & Strongly Agree \\
\hline 1 & 2 & 3 & 4 & 5 \\
\hline
\end{tabular}

Comments: 
3. I did not lose track of what the teacher was saying.

\begin{tabular}{|c|c|c|c|c|}
\hline $\begin{array}{l}\text { Strongly Dis- } \\
\text { agree }\end{array}$ & Disagree & $\begin{array}{l}\text { Neutral/No } \\
\text { opinion }\end{array}$ & Agree & Strongly Agree \\
\hline 1 & 2 & 3 & 4 & 5 \\
\hline
\end{tabular}

ments:

4. Because of the system, I payed more attention than I normally do in a regular class.

\begin{tabular}{|c|c|c|c|c|}
\hline $\begin{array}{l}\text { Strongly } \\
\text { agree }\end{array}$ & Disagree & $\begin{array}{l}\text { Neutral/No } \\
\text { opinion }\end{array}$ & Agree & Strongly Agree \\
\hline 1 & 2 & 3 & 4 & 5 \\
\hline
\end{tabular}

Comments:

5. I felt that the teacher paid more attention to me than he/she normally does in regular classes.

\begin{tabular}{|c|c|c|c|c|}
\hline $\begin{array}{l}\text { Strongly Dis- } \\
\text { agree }\end{array}$ & Disagree & $\begin{array}{l}\text { Neutral/No } \\
\text { opinion }\end{array}$ & Agree & Strongly Agree \\
\hline 1 & 2 & 3 & 4 & 5 \\
\hline
\end{tabular}

Comments: 
6. I've taken a course that covered the content of this mini-course.

\begin{tabular}{|c|c|l|c|c|}
\hline $\begin{array}{l}\text { Strongly Dis- } \\
\text { agree }\end{array}$ & Disagree & $\begin{array}{l}\text { Neutral/No } \\
\text { opinion }\end{array}$ & Agree & Strongly Agree \\
\hline 1 & 2 & 3 & 4 & 5 \\
\hline
\end{tabular}

Comments:

7. I was already familiar with the mathematics (trigonometry) used in the class.

\begin{tabular}{|cc|c|c|c|c|}
\hline $\begin{array}{l}\text { Strongly } \\
\text { agree }\end{array}$ & Dis- & Disagree & $\begin{array}{l}\text { Neutral/No } \\
\text { opinion }\end{array}$ & Agree & Strongly Agree \\
\hline 1 & 2 & 3 & 4 & 5 \\
\hline
\end{tabular}

Comments:

8. The instruction material was formatted properly.

\begin{tabular}{|c|c|l|c|c|}
\hline $\begin{array}{l}\text { Strongly Dis- } \\
\text { agree }\end{array}$ & Disagree & $\begin{array}{l}\text { Neutral/No } \\
\text { opinion }\end{array}$ & Agree & Strongly Agree \\
\hline 1 & 2 & 3 & 4 & 5 \\
\hline
\end{tabular}

Comments: 
9. I asked more questions that I normally do. Please tell us why.

\begin{tabular}{|c|c|c|c|c|}
\hline $\begin{array}{l}\text { Strongly Dis- } \\
\text { agree }\end{array}$ & Disagree & $\begin{array}{l}\text { Neutral/No } \\
\text { opinion }\end{array}$ & Agree & Strongly Agree \\
\hline 1 & 2 & 3 & 4 & 5 \\
\hline
\end{tabular}

Comments:

10. I would feel more comfortable if there were only blind students in the classroom.

\begin{tabular}{|c|c|c|c|c|}
\hline $\begin{array}{ll}\begin{array}{l}\text { Strongly } \\
\text { agree }\end{array} & \text { Dis- } \\
\text { - }\end{array}$ & Disagree & $\begin{array}{l}\text { Neutral/No } \\
\text { opinion }\end{array}$ & Agree & Strongly Agree \\
\hline 1 & 2 & 3 & 4 & 5 \\
\hline
\end{tabular}

Comments:

11. If the final oral exam were for real, I would need a tutor to help me studying. Please grade your need for a tutor: 1 being the lowest need, 5 for the highest.

\begin{tabular}{|c|c|l|l|c|}
\hline $\begin{array}{l}\text { I definitely need } \\
\text { a tutor }\end{array}$ & $\begin{array}{l}\text { I might need a } \\
\text { tutor }\end{array}$ & $\begin{array}{l}\text { Neutral/No } \\
\text { opinion }\end{array}$ & $\begin{array}{l}\text { I might not need } \\
\text { a tutor }\end{array}$ & $\begin{array}{l}\text { I definitely do } \\
\text { not need a tutor }\end{array}$ \\
\hline 1 & 2 & 3 & 4 & 5 \\
\hline
\end{tabular}

Comments:

12. I could clearly understand the graphics used in this mini-course. 


\begin{tabular}{|c|c|l|c|c|}
\hline $\begin{array}{l}\text { Strongly Dis- } \\
\text { agree }\end{array}$ & Disagree & $\begin{array}{l}\text { Neutral/No } \\
\text { opinion }\end{array}$ & Agree & Strongly Agree \\
\hline 1 & 2 & 3 & 4 & 5 \\
\hline
\end{tabular}

Justification:

13. It was easy for me to understand what the teacher was saying when the discussion used a figure.

\begin{tabular}{|c|c|l|c|c|}
\hline $\begin{array}{l}\text { Strongly Dis- } \\
\text { agree }\end{array}$ & Disagree & $\begin{array}{l}\text { Neutral/No } \\
\text { opinion }\end{array}$ & Agree & Strongly Agree \\
\hline 1 & 2 & 3 & 4 & 5 \\
\hline
\end{tabular}

Comments:

14. I was able to explore other portions of the graphical material used in this mini-course and still keep up with the lecture.

\begin{tabular}{|c|c|l|c|c|}
\hline $\begin{array}{l}\text { Strongly Dis- } \\
\text { agree }\end{array}$ & Disagree & $\begin{array}{l}\text { Neutral/No } \\
\text { opinion }\end{array}$ & Agree & Strongly Agree \\
\hline 1 & 2 & 3 & 4 & 5 \\
\hline
\end{tabular}

Comments:

15. I was able to understand the labels in the figures.

\begin{tabular}{|c|c|c|c|c|}
\hline $\begin{array}{l}\text { Strongly Dis- } \\
\text { agree }\end{array}$ & Disagree & $\begin{array}{l}\text { Neutral/No } \\
\text { opinion }\end{array}$ & Agree & Strongly Agree \\
\hline 1 & 2 & 3 & 4 & 5 \\
\hline
\end{tabular}


Comments:

16. Because of what the teacher said, I could move my reading hand directly to the point in the figure he/she was referring to.

\begin{tabular}{|c|c|c|c|c|}
\hline $\begin{array}{l}\text { Strongly Dis- } \\
\text { agree }\end{array}$ & Disagree & $\begin{array}{l}\text { Neutral/No } \\
\text { opinion }\end{array}$ & Agree & Strongly Agree \\
\hline 1 & 2 & 3 & 4 & 5 \\
\hline
\end{tabular}

Comments: 
17. I felt more able to ask questions (related to the lesson) than in a regular class.

\begin{tabular}{|c|c|c|c|c|}
\hline $\begin{array}{l}\text { Strongly } \\
\text { agree }\end{array}$ & Disagree & $\begin{array}{l}\text { Neutral/No } \\
\text { opinion }\end{array}$ & Agree & Strongly Agree \\
\hline 1 & 2 & 3 & 4 & 5 \\
\hline
\end{tabular}

Comments:

18. I was comfortable using the system in the class.

\begin{tabular}{|c|c|c|c|c|}
\hline $\begin{array}{ll}\begin{array}{l}\text { Strongly } \\
\text { agree }\end{array} & \text { Dis- }\end{array}$ & Disagree & $\begin{array}{l}\text { Neutral/No } \\
\text { opinion }\end{array}$ & Agree & Strongly Agree \\
\hline 1 & 2 & 3 & 4 & 5 \\
\hline
\end{tabular}

Comments:

19. Keeping up with the class along with the graphics is tiring.

\begin{tabular}{|c|c|l|c|c|}
\hline $\begin{array}{l}\text { Strongly Dis- } \\
\text { agree }\end{array}$ & Disagree & $\begin{array}{l}\text { Neutral/No } \\
\text { opinion }\end{array}$ & Agree & Strongly Agree \\
\hline 1 & 2 & 3 & 4 & 5 \\
\hline
\end{tabular}

Comments:

20. During the exam, I could remember the figures in my notes. 


\begin{tabular}{|c|c|l|c|c|}
\hline $\begin{array}{l}\text { Strongly Dis- } \\
\text { agree }\end{array}$ & Disagree & $\begin{array}{l}\text { Neutral/No } \\
\text { opinion }\end{array}$ & Agree & Strongly Agree \\
\hline 1 & 2 & 3 & 4 & 5 \\
\hline
\end{tabular}

Comments:

21. I felt comfortable using the glove while attending to class.

\begin{tabular}{|c|c|c|c|c|}
\hline $\begin{array}{l}\text { Strongly } \\
\text { agree }\end{array}$ & Disagree & $\begin{array}{l}\text { Neutral/No } \\
\text { opinion }\end{array}$ & Agree & Strongly Agree \\
\hline 1 & 2 & 3 & 4 & 5 \\
\hline
\end{tabular}

Comments:

22. The glove was not an impediment for keeping up with the lecture.

\begin{tabular}{|cc|c|l|c|c|}
\hline $\begin{array}{l}\text { Strongly } \\
\text { agree }\end{array}$ & Dis- & Disagree & $\begin{array}{l}\text { Neutral/No } \\
\text { opinion }\end{array}$ & Agree & Strongly Agree \\
\hline 1 & 2 & 3 & 4 & 5 \\
\hline
\end{tabular}

Comments: 
23. I felt that the system was a distraction instead of a help in attending to the instruction.

\begin{tabular}{|c|c|c|c|c|}
\hline $\begin{array}{l}\text { Strongly Dis- } \\
\text { agree }\end{array}$ & Disagree & $\begin{array}{l}\text { Neutral/No } \\
\text { opinion }\end{array}$ & Agree & Strongly Agree \\
\hline 1 & 2 & 3 & 4 & 5 \\
\hline
\end{tabular}

Comments:

24. The system disrupted the flow of the lecture.

\begin{tabular}{|c|c|c|c|c|}
\hline $\begin{array}{l}\text { Strongly Dis- } \\
\text { agree }\end{array}$ & Disagree & $\begin{array}{l}\text { Neutral/No } \\
\text { opinion }\end{array}$ & Agree & Strongly Agree \\
\hline 1 & 2 & 3 & 4 & 5 \\
\hline
\end{tabular}

\section{Comments:}

25. Playing the game helped in attending the lecture.

\begin{tabular}{|c|c|c|c|c|}
\hline $\begin{array}{l}\text { Strongly Dis- } \\
\text { agree }\end{array}$ & Disagree & $\begin{array}{l}\text { Neutral/No } \\
\text { opinion }\end{array}$ & Agree & Strongly Agree \\
\hline 1 & 2 & 3 & 4 & 5 \\
\hline
\end{tabular}

Comments: 
26. Playing the charade helped in attending the lecture.

\begin{tabular}{|c|c|c|c|c|}
\hline $\begin{array}{l}\text { Strongly Dis- } \\
\text { agree }\end{array}$ & Disagree & $\begin{array}{l}\text { Neutral/No } \\
\text { opinion }\end{array}$ & Agree & Strongly Agree \\
\hline 1 & 2 & 3 & 4 & 5 \\
\hline
\end{tabular}

Comments:

27. I was able to point at my chart and ask questions.

\begin{tabular}{|cc|c|c|c|c|}
\hline $\begin{array}{l}\text { Strongly } \\
\text { agree }\end{array}$ & Dis- & Disagree & $\begin{array}{l}\text { Neutral/No } \\
\text { opinion }\end{array}$ & Agree & Strongly Agree \\
\hline 1 & 2 & 3 & 4 & 5 \\
\hline
\end{tabular}

Comments:

28. I think that because of the system, I will get a better grade in the oral exam.

\begin{tabular}{|c|c|l|c|c|}
\hline $\begin{array}{l}\text { Strongly Dis- } \\
\text { agree }\end{array}$ & Disagree & $\begin{array}{l}\text { Neutral/No } \\
\text { opinion }\end{array}$ & Agree & Strongly Agree \\
\hline 1 & 2 & 3 & 4 & 5 \\
\hline
\end{tabular}

Comments: 
29. If this system were used in regular classrooms, the use of the cameras for tracking teacher and students who are blind would intimidate me.

\begin{tabular}{|cc|c|l|c|c|}
\hline $\begin{array}{l}\text { Strongly } \\
\text { agree }\end{array}$ & Dis- & Disagree & $\begin{array}{l}\text { Neutral/No } \\
\text { opinion }\end{array}$ & Agree & Strongly Agree \\
\hline 1 & 2 & 3 & 4 & 5 \\
\hline
\end{tabular}

Comments:

Please answer the following questions only if you have finished the second mini-course.

30. If this system were used in regular classrooms, the use of the cameras for tracking teacher and students who are blind would intimidate me.

\begin{tabular}{|c|c|c|c|c|}
\hline $\begin{array}{ll}\begin{array}{l}\text { Strongly } \\
\text { agree }\end{array} & \text { Dis- } \\
\text { agres }\end{array}$ & Disagree & $\begin{array}{l}\text { Neutral/No } \\
\text { opinion }\end{array}$ & Agree & Strongly Agree \\
\hline 1 & 2 & 3 & 4 & 5 \\
\hline
\end{tabular}

Comments:

31. My overall understanding of the concepts covered was better in the mini-course I took with the help of the system than in the one I took without the help of the system.

\begin{tabular}{|cc|c|c|c|c|}
\hline $\begin{array}{l}\text { Strongly } \\
\text { agree }\end{array}$ & Dis- & Disagree & $\begin{array}{l}\text { Neutral/No } \\
\text { opinion }\end{array}$ & Agree & Strongly Agree \\
\hline 1 & 2 & 3 & 4 & 5 \\
\hline
\end{tabular}

Comments: 
32. I believe the system will be useful in real world teaching.

\begin{tabular}{|c|c|c|c|c|}
\hline $\begin{array}{l}\text { Strongly Dis- } \\
\text { agree }\end{array}$ & Disagree & $\begin{array}{l}\text { Neutral/No } \\
\text { opinion }\end{array}$ & Agree & Strongly Agree \\
\hline 1 & 2 & 3 & 4 & 5 \\
\hline
\end{tabular}

Comments:

33. Attending the class using the system is more stressful than without it.

\begin{tabular}{|c|c|c|c|c|}
\hline $\begin{array}{ll}\begin{array}{l}\text { Strongly } \\
\text { agree }\end{array} & \text { Dis- } \\
\text { - }\end{array}$ & Disagree & $\begin{array}{l}\text { Neutral/No } \\
\text { opinion }\end{array}$ & Agree & Strongly Agree \\
\hline 1 & 2 & 3 & 4 & 5 \\
\hline
\end{tabular}

Comments:

34. Using the system made more tired than not using it.

\begin{tabular}{|c|c|l|c|c|}
\hline $\begin{array}{l}\text { Strongly Dis- } \\
\text { agree }\end{array}$ & Disagree & $\begin{array}{l}\text { Neutral/No } \\
\text { opinion }\end{array}$ & Agree & Strongly Agree \\
\hline 1 & 2 & 3 & 4 & 5 \\
\hline
\end{tabular}

Comments:

35. The use of the system slowed down the lecture more than having blind students in the class who do not have the system. 


\begin{tabular}{|c|c|l|c|c|}
\hline $\begin{array}{l}\text { Strongly Dis- } \\
\text { agree }\end{array}$ & Disagree & $\begin{array}{l}\text { Neutral/No } \\
\text { opinion }\end{array}$ & Agree & Strongly Agree \\
\hline 1 & 2 & 3 & 4 & 5 \\
\hline
\end{tabular}

Comments:

36. Please give us suggestions to improve our system. 
This post - questionnaire is supposed to be answered by the STUDENTS WHO ARE BLIND who just attended to a course where the augmentative technology NOT was employed and took the post-test

\section{Please answer the following questions}

Student who is blind \#:

1. The course flowed as any other regular course.

\begin{tabular}{|c|c|c|c|c|}
\hline $\begin{array}{l}\text { Strongly } \\
\text { agree }\end{array}$ & Disagree & $\begin{array}{l}\text { Neutral/No } \\
\text { opinion }\end{array}$ & Agree & Strongly Agree \\
\hline 1 & 2 & 3 & 4 & 5 \\
\hline
\end{tabular}

Comments:

2. Having access to the instructional material before class helped me in keeping up with the lecture.

\begin{tabular}{|c|c|l|c|c|}
\hline $\begin{array}{l}\text { Strongly Dis- } \\
\text { agree }\end{array}$ & Disagree & $\begin{array}{l}\text { Neutral/No } \\
\text { opinion }\end{array}$ & Agree & Strongly Agree \\
\hline 1 & 2 & 3 & 4 & 5 \\
\hline
\end{tabular}

Comments:

3. I did not lose track of what the teacher was saying.

\begin{tabular}{|c|c|c|c|c|}
\hline $\begin{array}{l}\text { Strongly } \\
\text { agree }\end{array}$ & Disagree & $\begin{array}{l}\text { Neutral/No } \\
\text { opinion }\end{array}$ & Agree & Strongly Agree \\
\hline 1 & 2 & 3 & 4 & 5 \\
\hline
\end{tabular}

Comments: 
4. I payed more attention than I normally do in a regular class.

\begin{tabular}{|c|c|l|c|c|}
\hline $\begin{array}{l}\text { Strongly Dis- } \\
\text { agree }\end{array}$ & Disagree & $\begin{array}{l}\text { Neutral/No } \\
\text { opinion }\end{array}$ & Agree & Strongly Agree \\
\hline 1 & 2 & 3 & 4 & 5 \\
\hline
\end{tabular}

Comments:

5. I felt that the teacher paid more attention to me than he/she normally does in regular classes.

\begin{tabular}{|c|c|c|c|c|}
\hline $\begin{array}{lc}\begin{array}{l}\text { Strongly } \\
\text { agree }\end{array} & \text { Dis- } \\
\end{array}$ & Disagree & $\begin{array}{l}\text { Neutral/No } \\
\text { opinion }\end{array}$ & Agree & Strongly Agree \\
\hline 1 & 2 & 3 & 4 & 5 \\
\hline
\end{tabular}

Comments:

6. I've taken a course that covered the content of this mini-course.

\begin{tabular}{|c|c|c|c|c|}
\hline $\begin{array}{l}\text { Strongly } \\
\text { agree }\end{array}$ & Disagree & $\begin{array}{l}\text { Neutral/No } \\
\text { opinion }\end{array}$ & Agree & Strongly Agree \\
\hline 1 & 2 & 3 & 4 & 5 \\
\hline
\end{tabular}

Comments: 
7. I was already familiar with the mathematics (trigonometry) used in the class.

\begin{tabular}{|c|c|c|c|c|}
\hline $\begin{array}{ll}\text { Strongly } & \text { Dis- } \\
\text { agree } & \end{array}$ & Disagree & $\begin{array}{l}\text { Neutral/No } \\
\text { opinion }\end{array}$ & Agree & Strongly Agree \\
\hline 1 & 2 & 3 & 4 & 5 \\
\hline
\end{tabular}

Comments:

8. The instruction material was formatted properly.

\begin{tabular}{|c|c|l|c|c|}
\hline $\begin{array}{l}\text { Strongly Dis- } \\
\text { agree }\end{array}$ & Disagree & $\begin{array}{l}\text { Neutral/No } \\
\text { opinion }\end{array}$ & Agree & Strongly Agree \\
\hline 1 & 2 & 3 & 4 & 5 \\
\hline
\end{tabular}

Comments:

9. I asked more questions that I normally do. Please tell us why.

\begin{tabular}{|cc|c|c|c|c|}
\hline $\begin{array}{l}\text { Strongly } \\
\text { agree }\end{array}$ & Dis- & Disagree & $\begin{array}{l}\text { Neutral/No } \\
\text { opinion }\end{array}$ & Agree & Strongly Agree \\
\hline 1 & 2 & 3 & 4 & 5 \\
\hline
\end{tabular}

Comments:

10. I would feel more comfortable if there were students who were blind in the classroom. 


\begin{tabular}{|c|c|l|c|c|}
\hline $\begin{array}{l}\text { Strongly Dis- } \\
\text { agree }\end{array}$ & Disagree & $\begin{array}{l}\text { Neutral/No } \\
\text { opinion }\end{array}$ & Agree & Strongly Agree \\
\hline 1 & 2 & 3 & 4 & 5 \\
\hline
\end{tabular}

Comments:

11. If the final oral exam were for real, I would need a tutor to help me studying. Please grade your need for a tutor: 1 being the lowest need, 5 for the highest.

\begin{tabular}{|c|c|l|c|c|}
\hline $\begin{array}{l}\text { I definitely need } \\
\text { a tutor }\end{array}$ & $\begin{array}{l}\text { I might need a } \\
\text { tutor }\end{array}$ & $\begin{array}{l}\text { Neutral/No } \\
\text { opinion }\end{array}$ & $\begin{array}{l}\text { I might not need } \\
\text { a tutor }\end{array}$ & $\begin{array}{l}\text { I definitely do } \\
\text { not need a tutor }\end{array}$ \\
\hline 1 & 2 & 3 & 4 & 5 \\
\hline
\end{tabular}

Comments:

12. I could clearly understand the graphics used in this mini-course.

\begin{tabular}{|c|c|c|c|c|}
\hline $\begin{array}{l}\text { Strongly Dis- } \\
\text { agree }\end{array}$ & Disagree & $\begin{array}{l}\text { Neutral/No } \\
\text { opinion }\end{array}$ & Agree & Strongly Agree \\
\hline 1 & 2 & 3 & 4 & 5 \\
\hline
\end{tabular}

Justification:

13. It was easy for me to understand what the teacher was saying when the discussion used a figure. 


\begin{tabular}{|c|c|l|c|c|}
\hline $\begin{array}{l}\text { Strongly Dis- } \\
\text { agree }\end{array}$ & Disagree & $\begin{array}{l}\text { Neutral/No } \\
\text { opinion }\end{array}$ & Agree & Strongly Agree \\
\hline 1 & 2 & 3 & 4 & 5 \\
\hline
\end{tabular}

Comments:

14. I was able to explore other portions of the graphical material used in this mini-course and still keep up with the lecture.

\begin{tabular}{|c|c|l|c|c|}
\hline $\begin{array}{l}\text { Strongly Dis- } \\
\text { agree }\end{array}$ & Disagree & $\begin{array}{l}\text { Neutral/No } \\
\text { opinion }\end{array}$ & Agree & Strongly Agree \\
\hline 1 & 2 & 3 & 4 & 5 \\
\hline
\end{tabular}

Comments:

15. I was able to understand the labels in the figures.

\begin{tabular}{|c|c|c|c|c|}
\hline $\begin{array}{ll}\begin{array}{l}\text { Strongly } \\
\text { agree }\end{array} & \text { Dis- } \\
\end{array}$ & Disagree & $\begin{array}{l}\text { Neutral/No } \\
\text { opinion }\end{array}$ & Agree & Strongly Agree \\
\hline 1 & 2 & 3 & 4 & 5 \\
\hline
\end{tabular}

Comments:

16. Because of what the teacher said, I could move my reading hand directly to the point in the figure he/she was referring to. 


\begin{tabular}{|c|c|l|c|c|}
\hline $\begin{array}{l}\text { Strongly Dis- } \\
\text { agree }\end{array}$ & Disagree & $\begin{array}{l}\text { Neutral/No } \\
\text { opinion }\end{array}$ & Agree & Strongly Agree \\
\hline 1 & 2 & 3 & 4 & 5 \\
\hline
\end{tabular}

Comments:

17. I felt better able to ask questions (related to the lesson) than in a regular class.

\begin{tabular}{|c|c|c|c|c|}
\hline $\begin{array}{ll}\begin{array}{l}\text { Strongly } \\
\text { agree }\end{array} & \text { Dis- } \\
\text { agre }\end{array}$ & Disagree & $\begin{array}{l}\text { Neutral/No } \\
\text { opinion }\end{array}$ & Agree & Strongly Agree \\
\hline 1 & 2 & 3 & 4 & 5 \\
\hline
\end{tabular}

Comments:

18. Keeping up with the class along with the graphics is tiring.

\begin{tabular}{|c|c|c|c|c|}
\hline $\begin{array}{l}\text { Strongly Dis- } \\
\text { agree }\end{array}$ & Disagree & $\begin{array}{l}\text { Neutral/No } \\
\text { opinion }\end{array}$ & Agree & Strongly Agree \\
\hline 1 & 2 & 3 & 4 & 5 \\
\hline
\end{tabular}

Comments:

19. During the exam, I could remember the figures in my notes.

\begin{tabular}{|c|c|l|c|c|}
\hline $\begin{array}{l}\text { Strongly Dis- } \\
\text { agree }\end{array}$ & Disagree & $\begin{array}{l}\text { Neutral/No } \\
\text { opinion }\end{array}$ & Agree & Strongly Agree \\
\hline 1 & 2 & 3 & 4 & 5 \\
\hline
\end{tabular}


Comments:

20. I was able to point at my chart and ask questions.

\begin{tabular}{|c|c|c|c|c|}
\hline $\begin{array}{l}\text { Strongly Dis- } \\
\text { agree }\end{array}$ & Disagree & $\begin{array}{l}\text { Neutral/No } \\
\text { opinion }\end{array}$ & Agree & Strongly Agree \\
\hline 1 & 2 & 3 & 4 & 5 \\
\hline
\end{tabular}

Comments:

Please answer the following questions only if you have finished the second mini-course.

21. My overall understanding of the concepts covered was better in the mini-course I took with the help of the system than in the one I took without the help of the system.

\begin{tabular}{|c|c|c|c|c|}
\hline $\begin{array}{l}\text { Strongly Dis- } \\
\text { agree }\end{array}$ & Disagree & $\begin{array}{l}\text { Neutral/No } \\
\text { opinion }\end{array}$ & Agree & Strongly Agree \\
\hline 1 & 2 & 3 & 4 & 5 \\
\hline
\end{tabular}

Comments:

22. I believe the system will be useful in real world teaching.

\begin{tabular}{|c|c|c|c|c|}
\hline $\begin{array}{l}\text { Strongly Dis- } \\
\text { agree }\end{array}$ & Disagree & $\begin{array}{l}\text { Neutral/No } \\
\text { opinion }\end{array}$ & Agree & Strongly Agree \\
\hline 1 & 2 & 3 & 4 & 5 \\
\hline
\end{tabular}


Comments:

23. Attending the class using the system is more stressful than without it.

\begin{tabular}{|c|c|c|c|c|}
\hline $\begin{array}{l}\text { Strongly Dis- } \\
\text { agree }\end{array}$ & Disagree & $\begin{array}{l}\text { Neutral/No } \\
\text { opinion }\end{array}$ & Agree & Strongly Agree \\
\hline 1 & 2 & 3 & 4 & 5 \\
\hline
\end{tabular}

Comments:

24. Using the system made me more tired than not using it.

\begin{tabular}{|c|c|c|c|c|}
\hline $\begin{array}{l}\text { Strongly Dis- } \\
\text { agree }\end{array}$ & Disagree & $\begin{array}{l}\text { Neutral/No } \\
\text { opinion }\end{array}$ & Agree & Strongly Agree \\
\hline 1 & 2 & 3 & 4 & 5 \\
\hline
\end{tabular}

Comments:

25. The use of the system slowed down the lecture more than having students who are blind in the class who do not have the system.

\begin{tabular}{|c|c|c|c|c|}
\hline $\begin{array}{l}\text { Strongly Dis- } \\
\text { agree }\end{array}$ & Disagree & $\begin{array}{l}\text { Neutral/No } \\
\text { opinion }\end{array}$ & Agree & Strongly Agree \\
\hline 1 & 2 & 3 & 4 & 5 \\
\hline
\end{tabular}

Comments: 
26. Please give us suggestions to improve our system. 
This post - questionnaire is supposed to be answered by the sighted students who just attended to a course where the augmentative technology was employed and took the post-test

\section{Please answer the following questions}

Sighted Student \#:

1. The course flowed as any other regular course.

\begin{tabular}{|c|c|c|c|c|}
\hline $\begin{array}{ll}\begin{array}{l}\text { Strongly } \\
\text { agree }\end{array} & \text { Dis- } \\
\end{array}$ & Disagree & $\begin{array}{l}\text { Neutral/No } \\
\text { opinion }\end{array}$ & Agree & Strongly Agree \\
\hline 1 & 2 & 3 & 4 & 5 \\
\hline
\end{tabular}

ments:

2. Having access to the instructional material before class helped me in keeping up with the lecture.

\begin{tabular}{|c|c|c|c|c|}
\hline $\begin{array}{ll}\begin{array}{l}\text { Strongly } \\
\text { agree }\end{array} & \text { Dis- } \\
\end{array}$ & Disagree & $\begin{array}{l}\text { Neutral/No } \\
\text { opinion }\end{array}$ & Agree & Strongly Agree \\
\hline 1 & 2 & 3 & 4 & 5 \\
\hline
\end{tabular}

ments: 
3. I payed more attention than I normally do in a regular class.

\begin{tabular}{|c|c|l|c|c|}
\hline $\begin{array}{l}\text { Strongly Dis- } \\
\text { agree }\end{array}$ & Disagree & $\begin{array}{l}\text { Neutral/No } \\
\text { opinion }\end{array}$ & Agree & Strongly Agree \\
\hline 1 & 2 & 3 & 4 & 5 \\
\hline
\end{tabular}

Comments:

4. I've taken a course that covered the content of this mini-course.

\begin{tabular}{|c|c|l|l|c|}
\hline $\begin{array}{l}\text { Strongly Dis- } \\
\text { agree }\end{array}$ & Disagree & $\begin{array}{l}\text { Neutral/No } \\
\text { opinion }\end{array}$ & Agree & Strongly Agree \\
\hline 1 & 2 & 3 & 4 & 5 \\
\hline
\end{tabular}

ments:

5. I was already familiar with the mathematics (trigonometry) used in the class.

\begin{tabular}{|c|c|c|c|c|}
\hline $\begin{array}{l}\text { Strongly Dis- } \\
\text { agree }\end{array}$ & Disagree & $\begin{array}{l}\text { Neutral/No } \\
\text { opinion }\end{array}$ & Agree & Strongly Agree \\
\hline 1 & 2 & 3 & 4 & 5 \\
\hline
\end{tabular}

Comments: 
6. The instructional material was formatted properly.

\begin{tabular}{|c|c|c|c|c|}
\hline $\begin{array}{ll}\text { Strongly } & \text { Dis- } \\
\text { agree } & \end{array}$ & Disagree & $\begin{array}{l}\text { Neutral/No } \\
\text { opinion }\end{array}$ & Agree & Strongly Agree \\
\hline 1 & 2 & 3 & 4 & 5 \\
\hline
\end{tabular}

Comments:

7. I asked more questions than I normally do. Please tell us why.

\begin{tabular}{|c|c|l|c|c|}
\hline $\begin{array}{l}\text { Strongly Dis- } \\
\text { agree }\end{array}$ & Disagree & $\begin{array}{l}\text { Neutral/No } \\
\text { opinion }\end{array}$ & Agree & Strongly Agree \\
\hline 1 & 2 & 3 & 4 & 5 \\
\hline
\end{tabular}

Comments:

8. I could clearly understand the graphics used in this mini-course.

\begin{tabular}{|c|c|c|c|c|}
\hline $\begin{array}{l}\text { Strongly Dis- } \\
\text { agree }\end{array}$ & Disagree & $\begin{array}{l}\text { Neutral/No } \\
\text { opinion }\end{array}$ & Agree & Strongly Agree \\
\hline 1 & 2 & 3 & 4 & 5 \\
\hline
\end{tabular}

Comments: 
9. It was easy for me to understand what the teacher was saying when the discussion was around a figure.

\begin{tabular}{|c|c|l|c|c|}
\hline $\begin{array}{l}\text { Strongly Dis- } \\
\text { agree }\end{array}$ & Disagree & $\begin{array}{l}\text { Neutral/No } \\
\text { opinion }\end{array}$ & Agree & Strongly Agree \\
\hline 1 & 2 & 3 & 4 & 5 \\
\hline
\end{tabular}

Comments:

10. I was able to explore other portions of the graphical material used in this mini-course and still keep up with the lecture.

\begin{tabular}{|c|c|l|c|c|}
\hline $\begin{array}{l}\text { Strongly Dis- } \\
\text { agree }\end{array}$ & Disagree & $\begin{array}{l}\text { Neutral/No } \\
\text { opinion }\end{array}$ & Agree & Strongly Agree \\
\hline 1 & 2 & 3 & 4 & 5 \\
\hline
\end{tabular}

Comments:

11. I was able to understand the labels in the figures.

\begin{tabular}{|c|c|c|c|c|}
\hline $\begin{array}{l}\text { Strongly Dis- } \\
\text { agree }\end{array}$ & Disagree & $\begin{array}{l}\text { Neutral/No } \\
\text { opinion }\end{array}$ & Agree & Strongly Agree \\
\hline 1 & 2 & 3 & 4 & 5 \\
\hline
\end{tabular}

Comments:

12. Keeping up with the class along with the graphics is tiring.

\begin{tabular}{|c|c|c|c|c|}
\hline $\begin{array}{ll}\text { Strongly } & \text { Dis- } \\
\text { agree } & \end{array}$ & Disagree & $\begin{array}{l}\text { Neutral/No } \\
\text { opinion }\end{array}$ & Agree & Strongly Agree \\
\hline 1 & 2 & 3 & 4 & 5 \\
\hline
\end{tabular}


Comments:

13. During the exam, I could remember the figures on my notes.

\begin{tabular}{|c|c|c|c|c|}
\hline $\begin{array}{l}\text { Strongly Dis- } \\
\text { agree }\end{array}$ & Disagree & $\begin{array}{l}\text { Neutral/No } \\
\text { opinion }\end{array}$ & Agree & Strongly Agree \\
\hline 1 & 2 & 3 & 4 & 5 \\
\hline
\end{tabular}

Justification:

14. The teacher's dysplay made the teacher pay less attention to me.

\begin{tabular}{|c|c|c|c|c|}
\hline $\begin{array}{l}\text { Strongly Dis- } \\
\text { agree }\end{array}$ & Disagree & $\begin{array}{l}\text { Neutral/No } \\
\text { opinion }\end{array}$ & Agree & Strongly Agree \\
\hline 1 & 2 & 3 & 4 & 5 \\
\hline
\end{tabular}

Comments:

15. The system disrupted the flow of the lecture.

\begin{tabular}{|c|c|c|c|c|}
\hline $\begin{array}{l}\text { Strongly Dis- } \\
\text { agree }\end{array}$ & Disagree & $\begin{array}{l}\text { Neutral/No } \\
\text { opinion }\end{array}$ & Agree & Strongly Agree \\
\hline 1 & 2 & 3 & 4 & 5 \\
\hline
\end{tabular}

Comments:

16. If this system ever make it to classrooms, the use of the cameras for tracking teacher and students who are blind would intimidate me. 


\begin{tabular}{|c|c|l|c|c|}
\hline $\begin{array}{l}\text { Strongly Dis- } \\
\text { agree }\end{array}$ & Disagree & $\begin{array}{l}\text { Neutral/No } \\
\text { opinion }\end{array}$ & Agree & Strongly Agree \\
\hline 1 & 2 & 3 & 4 & 5 \\
\hline
\end{tabular}

Comments: 
Please answer the following questions only if you have finished the second mini-course.

17. Please rate your preference between both teaching alternatives. You can assign a number ranging from 1 to 5 . 1 being that you definitely prefer attending classes without the system, 2 that you are more inclined to classes without the system, 3 that you are indifferent, 4 that you are more inclined for classes with the augmentative system, 5 that you definitely prefer attending classes with the augmentative system. Please justify your answer.

\begin{tabular}{|c|c|c|c|c|}
\hline $\begin{array}{l}\text { Definitely with- } \\
\text { out the augmen- } \\
\text { tative system }\end{array}$ & $\begin{array}{l}\text { More inclined to } \\
\text { classes without } \\
\text { the augmenta- } \\
\text { tive system }\end{array}$ & $\begin{array}{l}\text { Neutral/No } \\
\text { opinion }\end{array}$ & $\begin{array}{l}\text { More inclined to } \\
\text { classes with the } \\
\text { augmentative } \\
\text { system }\end{array}$ & $\begin{array}{l}\text { Definitely } \\
\text { classes with the } \\
\text { augmentative } \\
\text { system }\end{array}$ \\
\hline 1 & 2 & 3 & 4 & 5 \\
\hline
\end{tabular}

Comments:

18. I feel that having a student in the class who is using the system disrupted the flow of the lectures more than having a student who is blind in the class who was not using the system.

\begin{tabular}{|c|c|c|c|c|}
\hline $\begin{array}{l}\text { Strongly Dis- } \\
\text { agree }\end{array}$ & Disagree & $\begin{array}{l}\text { Neutral/No } \\
\text { opinion }\end{array}$ & Agree & Strongly Agree \\
\hline 1 & 2 & 3 & 4 & 5 \\
\hline
\end{tabular}

Comments: 
19. I believe the system will be useful in real world teaching.

\begin{tabular}{|c|c|c|c|c|}
\hline $\begin{array}{ll}\text { Strongly } & \text { Dis- } \\
\text { agree } & \end{array}$ & Disagree & $\begin{array}{l}\text { Neutral/No } \\
\text { opinion }\end{array}$ & Agree & Strongly Agree \\
\hline 1 & 2 & 3 & 4 & 5 \\
\hline
\end{tabular}

Comments:

20. Please give us suggestions to improve our system. 
This post - questionnaire is supposed to be answered by the SIGHTED STUDENTS who just attended to a course where the augmentative technology was NOT employed and took the post-test.

Please answer the following questions

Sighted Student \#:

1. The course flowed as any other regular course.

\begin{tabular}{|c|c|l|c|c|}
\hline $\begin{array}{l}\text { Strongly Dis- } \\
\text { agree }\end{array}$ & Disagree & $\begin{array}{l}\text { Neutral/No } \\
\text { opinion }\end{array}$ & Agree & Strongly Agree \\
\hline 1 & 2 & 3 & 4 & 5 \\
\hline
\end{tabular}

Comments:

2. Having access to the instructional material before class helped me in keeping up with the lecture.

\begin{tabular}{|c|c|l|c|c|}
\hline $\begin{array}{l}\text { Strongly Dis- } \\
\text { agree }\end{array}$ & Disagree & $\begin{array}{l}\text { Neutral/No } \\
\text { opinion }\end{array}$ & Agree & Strongly Agree \\
\hline 1 & 2 & 3 & 4 & 5 \\
\hline
\end{tabular}

Comments: 
3. I payed more attention than I normally do in a regular class.

\begin{tabular}{|c|c|c|c|c|}
\hline $\begin{array}{l}\text { Strongly Dis- } \\
\text { agree }\end{array}$ & Disagree & $\begin{array}{l}\text { Neutral/No } \\
\text { opinion }\end{array}$ & Agree & Strongly Agree \\
\hline 1 & 2 & 3 & 4 & 5 \\
\hline
\end{tabular}

Comments:

4. I've taken a course that covered the content of this mini-course.

\begin{tabular}{|c|c|c|c|c|}
\hline $\begin{array}{l}\text { Strongly Dis- } \\
\text { agree }\end{array}$ & Disagree & $\begin{array}{l}\text { Neutral/No } \\
\text { opinion }\end{array}$ & Agree & Strongly Agree \\
\hline 1 & 2 & 3 & 4 & 5 \\
\hline
\end{tabular}

Comments:

5. I was already familiar with the mathematics (trigonometry) used in the class.

\begin{tabular}{|c|c|c|c|c|}
\hline $\begin{array}{l}\text { Strongly Dis- } \\
\text { agree }\end{array}$ & Disagree & $\begin{array}{l}\text { Neutral/No } \\
\text { opinion }\end{array}$ & Agree & Strongly Agree \\
\hline 1 & 2 & 3 & 4 & 5 \\
\hline
\end{tabular}

Comments: 
6. The instructional material was formatted properly.

\begin{tabular}{|c|c|c|c|c|}
\hline $\begin{array}{ll}\begin{array}{l}\text { Strongly } \\
\text { agree }\end{array} & \text { Dis- } \\
\text { agre }\end{array}$ & Disagree & $\begin{array}{l}\text { Neutral/No } \\
\text { opinion }\end{array}$ & Agree & Strongly Agree \\
\hline 1 & 2 & 3 & 4 & 5 \\
\hline
\end{tabular}

Comments:

7. I asked more questions than I normally do. Please tell us why.

\begin{tabular}{|c|c|l|c|c|}
\hline $\begin{array}{l}\text { Strongly Dis- } \\
\text { agree }\end{array}$ & Disagree & $\begin{array}{l}\text { Neutral/No } \\
\text { opinion }\end{array}$ & Agree & Strongly Agree \\
\hline 1 & 2 & 3 & 4 & 5 \\
\hline
\end{tabular}

Comments:

8. I could clearly understand the graphics used in this mini-course.

\begin{tabular}{|c|c|c|c|c|}
\hline $\begin{array}{ll}\begin{array}{l}\text { Strongly } \\
\text { agree }\end{array} & \text { Dis- }\end{array}$ & Disagree & $\begin{array}{l}\text { Neutral/No } \\
\text { opinion }\end{array}$ & Agree & Strongly Agree \\
\hline 1 & 2 & 3 & 4 & 5 \\
\hline
\end{tabular}

Comments: 
9. It was easy for me to understand what the teacher was saying when the discussion was around a figure.

\begin{tabular}{|c|c|l|c|c|}
\hline $\begin{array}{l}\text { Strongly Dis- } \\
\text { agree }\end{array}$ & Disagree & $\begin{array}{l}\text { Neutral/No } \\
\text { opinion }\end{array}$ & Agree & Strongly Agree \\
\hline 1 & 2 & 3 & 4 & 5 \\
\hline
\end{tabular}

Comments:

10. I was able to explore other portions of the graphical material used in this mini-course and still keep up with the lecture.

\begin{tabular}{|c|c|c|c|c|}
\hline $\begin{array}{ll}\begin{array}{l}\text { Strongly } \\
\text { agree }\end{array} & \text { Dis- }\end{array}$ & Disagree & $\begin{array}{l}\text { Neutral/No } \\
\text { opinion }\end{array}$ & Agree & Strongly Agree \\
\hline 1 & 2 & 3 & 4 & 5 \\
\hline
\end{tabular}

Comments:

11. I was able to understand the labels in the figures.

\begin{tabular}{|c|c|l|c|c|}
\hline $\begin{array}{l}\text { Strongly Dis- } \\
\text { agree }\end{array}$ & Disagree & $\begin{array}{l}\text { Neutral/No } \\
\text { opinion }\end{array}$ & Agree & Strongly Agree \\
\hline 1 & 2 & 3 & 4 & 5 \\
\hline
\end{tabular}

Comments:

12. Keeping up with the class along with the graphics is tiring. 


\begin{tabular}{|c|c|l|c|c|}
\hline $\begin{array}{l}\text { Strongly Dis- } \\
\text { agree }\end{array}$ & Disagree & $\begin{array}{l}\text { Neutral/No } \\
\text { opinion }\end{array}$ & Agree & Strongly Agree \\
\hline 1 & 2 & 3 & 4 & 5 \\
\hline
\end{tabular}

Comments:

13. During the exam, I could remember the figures on my notes.

\begin{tabular}{|c|c|l|c|c|}
\hline $\begin{array}{l}\text { Strongly Dis- } \\
\text { agree }\end{array}$ & Disagree & $\begin{array}{l}\text { Neutral/No } \\
\text { opinion }\end{array}$ & Agree & Strongly Agree \\
\hline 1 & 2 & 3 & 4 & 5 \\
\hline
\end{tabular}

Justification: 
Please answer the following questions only if you have finished the second mini-course.

14. Please rate your preference between both teaching alternatives. You can assign a number ranging from 1 to 5 . 1 being that you definitely prefer attending classes without the system, 2 that you are more inclined to classes without the system, 3 that you are indifferent, 4 that you are more inclined for classes with the augmentative system, 5 that you definitely prefer attending classes with the augmentative system. Please justify your answer.

\begin{tabular}{|c|c|c|c|c|}
\hline $\begin{array}{l}\text { Definitely with- } \\
\text { out the augmen- } \\
\text { tative system }\end{array}$ & $\begin{array}{l}\text { More inclined to } \\
\text { classes without } \\
\text { the augmenta- } \\
\text { tive system }\end{array}$ & $\begin{array}{l}\text { Neutral/No } \\
\text { opinion }\end{array}$ & $\begin{array}{l}\text { More inclined to } \\
\text { classes with the } \\
\text { augmentative } \\
\text { system }\end{array}$ & $\begin{array}{l}\text { Definitely } \\
\text { classes with the } \\
\text { augmentative } \\
\text { system }\end{array}$ \\
\hline 1 & 2 & 3 & 4 & 5 \\
\hline
\end{tabular}

Comments:

15. I feel that having blind students in the class using the system disrupted the flow of the lecture than having blind students without the system.

\begin{tabular}{|c|c|c|c|c|}
\hline $\begin{array}{l}\text { Strongly Dis- } \\
\text { agree }\end{array}$ & Disagree & $\begin{array}{l}\text { Neutral/No } \\
\text { opinion }\end{array}$ & Agree & Strongly Agree \\
\hline 1 & 2 & 3 & 4 & 5 \\
\hline
\end{tabular}

Comments: 
16. I believe the system will be useful in real world teaching.

\begin{tabular}{|c|c|c|c|c|}
\hline $\begin{array}{l}\text { Strongly Dis- } \\
\text { agree }\end{array}$ & Disagree & $\begin{array}{l}\text { Neutral/No } \\
\text { opinion }\end{array}$ & Agree & Strongly Agree \\
\hline 1 & 2 & 3 & 4 & 5 \\
\hline
\end{tabular}

Comments:

17. Please give us suggestions to improve our system. 
Table D.1: Average number of words per conversational turn (Complete Listing)

\begin{tabular}{|c|c|c|c|c|}
\hline Cur & Experimental Condition & Trial & Lesson & $\begin{array}{l}\text { Avg words } \\
\text { per turn } \\
\text { instructor }\end{array}$ \\
\hline \multirow[t]{18}{*}{ A } & \multirow[t]{3}{*}{ All sighted } & \multirow[t]{3}{*}{ Control group 1} & 1 & 11.38 \\
\hline & & & 2 & 11.84 \\
\hline & & & 3 & 9.21 \\
\hline & \multirow{6}{*}{ With the system } & \multirow[t]{3}{*}{$\mathrm{G}$} & 1 & 7.87 \\
\hline & & & 2 & 8.19 \\
\hline & & & 3 & 9.48 \\
\hline & & \multirow[t]{3}{*}{$\mathrm{N}$} & 1 & 12.70 \\
\hline & & & 2 & 10.89 \\
\hline & & & 3 & 9.98 \\
\hline & \multirow[t]{9}{*}{ Without the system } & \multirow[t]{3}{*}{$\mathrm{A}$} & 1 & 10.53 \\
\hline & & & 2 & 10.15 \\
\hline & & & 3 & 11.08 \\
\hline & & \multirow[t]{3}{*}{$\mathrm{O}$} & 1 & 11.21 \\
\hline & & & 2 & 12.43 \\
\hline & & & 3 & 12.73 \\
\hline & & \multirow[t]{3}{*}{$\mathrm{R}$} & 1 & 10.94 \\
\hline & & & 2 & 11.26 \\
\hline & & & 3 & 10.01 \\
\hline \multirow[t]{18}{*}{ B } & \multirow[t]{3}{*}{ All sighted } & \multirow[t]{3}{*}{ Control group 2} & 1 & 13.25 \\
\hline & & & 2 & 11.47 \\
\hline & & & 3 & 14.38 \\
\hline & \multirow[t]{9}{*}{ With the system } & \multirow[t]{3}{*}{ A } & 1 & 12.89 \\
\hline & & & 2 & 11.70 \\
\hline & & & 3 & 15.62 \\
\hline & & \multirow[t]{3}{*}{$\mathrm{O}$} & 1 & 15.57 \\
\hline & & & 2 & 14.87 \\
\hline & & & 3 & 20.60 \\
\hline & & \multirow[t]{3}{*}{$\mathrm{R}$} & 1 & 11.60 \\
\hline & & & 2 & 11.34 \\
\hline & & & 3 & 14.32 \\
\hline & \multirow[t]{6}{*}{ Without the system } & \multirow[t]{3}{*}{$\mathrm{G}$} & 1 & 15.17 \\
\hline & & & 2 & 14.59 \\
\hline & & & 3 & 15.67 \\
\hline & & \multirow[t]{3}{*}{$\mathrm{N}$} & 1 & 16.21 \\
\hline & & & 2 & 13.42 \\
\hline & & & 3 & 14.76 \\
\hline
\end{tabular}


Table D.2: Average of turn duration - Instructor (Complete listing)

\begin{tabular}{|c|c|c|c|c|}
\hline Cur & Experimental Condition & Trial & Lesson & $\begin{array}{r}\text { Avg duration of } \\
\text { instructor's turn } \\
\text { (in sec) }\end{array}$ \\
\hline \multirow[t]{18}{*}{ A } & \multirow{3}{*}{ All sighted } & \multirow{3}{*}{ Control group 1} & 1 & 6.11 \\
\hline & & & 2 & 6.45 \\
\hline & & & 3 & 5.24 \\
\hline & \multirow[t]{6}{*}{ With the system } & \multirow[t]{3}{*}{ G } & 1 & 6.05 \\
\hline & & & 2 & 4.58 \\
\hline & & & 3 & 4.62 \\
\hline & & \multirow[t]{3}{*}{$\mathrm{N}$} & 1 & 6.26 \\
\hline & & & 2 & 7.13 \\
\hline & & & 3 & 5.23 \\
\hline & \multirow[t]{9}{*}{ Without the system } & \multirow[t]{3}{*}{ A } & 1 & 5.54 \\
\hline & & & 2 & 4.83 \\
\hline & & & 3 & 6.3 \\
\hline & & \multirow[t]{3}{*}{$\mathrm{O}$} & 1 & 5.83 \\
\hline & & & 2 & 6.33 \\
\hline & & & 3 & 6.59 \\
\hline & & \multirow[t]{3}{*}{$\mathrm{R}$} & 1 & 5.34 \\
\hline & & & 2 & 5.55 \\
\hline & & & 3 & 5.61 \\
\hline \multirow[t]{18}{*}{ B } & \multirow[t]{3}{*}{ All sighted } & \multirow{3}{*}{ Control group 2} & 1 & 6.00 \\
\hline & & & 2 & 5.26 \\
\hline & & & 3 & 5.88 \\
\hline & \multirow[t]{9}{*}{ With the system } & \multirow[t]{3}{*}{ A } & 1 & 5.88 \\
\hline & & & 2 & 5.78 \\
\hline & & & 3 & 6.8 \\
\hline & & \multirow[t]{3}{*}{$\mathrm{O}$} & 1 & 6.77 \\
\hline & & & 2 & 6.26 \\
\hline & & & 3 & 8.42 \\
\hline & & \multirow[t]{3}{*}{$\mathrm{R}$} & 1 & 5.07 \\
\hline & & & 2 & 4.66 \\
\hline & & & 3 & 5.62 \\
\hline & \multirow[t]{6}{*}{ Without the system } & \multirow[t]{3}{*}{ G } & 1 & 6.42 \\
\hline & & & 2 & 6.22 \\
\hline & & & 3 & 5.98 \\
\hline & & \multirow[t]{3}{*}{$\mathrm{N}$} & 1 & 6.68 \\
\hline & & & 2 & 5.48 \\
\hline & & & 3 & 5.64 \\
\hline
\end{tabular}


Table D.3: Hand Positioning Events (Complete Listing)

\begin{tabular}{|c|c|c|c|c|}
\hline Cur & Experimental Condition & Trial & Lesson & Hand Position Events \\
\hline \multirow[t]{15}{*}{ A } & With the system & G & 1 & 0 \\
\hline & & & 2 & 0 \\
\hline & & & 3 & 0 \\
\hline & & $\mathrm{N}$ & 1 & 0 \\
\hline & & & 1 & 0 \\
\hline & & & 1 & 0 \\
\hline & Without the system & A & 1 & 3 \\
\hline & & & 2 & 6 \\
\hline & & & 3 & 5 \\
\hline & & $\mathrm{O}$ & 1 & 1 \\
\hline & & & 2 & 4 \\
\hline & & & 3 & 2 \\
\hline & & $\mathrm{R}$ & 1 & 1 \\
\hline & & & 2 & 3 \\
\hline & & & 3 & 1 \\
\hline \multirow[t]{15}{*}{ B } & With the system & A & 1 & 0 \\
\hline & & & 2 & 0 \\
\hline & & & 3 & 0 \\
\hline & & $\mathrm{O}$ & 1 & 0 \\
\hline & & & 2 & 2 \\
\hline & & & 3 & 0 \\
\hline & & $\mathrm{R}$ & 1 & 0 \\
\hline & & & 2 & 1 \\
\hline & & & 3 & 1 \\
\hline & Without the system & G & 1 & 0 \\
\hline & & & 2 & 1 \\
\hline & & & 3 & 1 \\
\hline & & $\mathrm{N}$ & 1 & 0 \\
\hline & & & 2 & 2 \\
\hline & & & 3 & 0 \\
\hline
\end{tabular}


Table D.4: Discourse Level - Complete listing

\begin{tabular}{|c|c|c|c|c|}
\hline Cur & Experimental Condition & Trial & Lesson & Percentage of object level turns \\
\hline \multirow[t]{18}{*}{ A } & \multirow[t]{3}{*}{ All sighted } & \multirow[t]{3}{*}{ Control group 1} & 1 & 95.85 \\
\hline & & & 2 & 98.00 \\
\hline & & & 3 & 97.60 \\
\hline & \multirow{6}{*}{ With the system } & \multirow[t]{3}{*}{$\mathrm{G}$} & 1 & 97.64 \\
\hline & & & 2 & 97.63 \\
\hline & & & 3 & 97.08 \\
\hline & & \multirow[t]{3}{*}{$\mathrm{N}$} & 0 & 99.34 \\
\hline & & & 1 & 96.43 \\
\hline & & & 2 & 93.21 \\
\hline & \multirow[t]{9}{*}{ Without the system } & \multirow[t]{3}{*}{$\mathrm{A}$} & 1 & 92.20 \\
\hline & & & 2 & 56.73 \\
\hline & & & 3 & 93.91 \\
\hline & & \multirow[t]{3}{*}{$\mathrm{O}$} & 1 & 82.82 \\
\hline & & & 2 & 72.30 \\
\hline & & & 3 & 97.96 \\
\hline & & \multirow[t]{3}{*}{$\mathrm{R}$} & 1 & 91.22 \\
\hline & & & 2 & 67.54 \\
\hline & & & 3 & 81.08 \\
\hline \multirow[t]{18}{*}{$\mathrm{B}$} & \multirow[t]{3}{*}{ All sighted } & \multirow[t]{3}{*}{ Control group 2} & 1 & 97.24 \\
\hline & & & 2 & 97.99 \\
\hline & & & 3 & 98.00 \\
\hline & \multirow[t]{9}{*}{ With the system } & \multirow[t]{3}{*}{ A } & 1 & 82.44 \\
\hline & & & 2 & 84.01 \\
\hline & & & 3 & 97.24 \\
\hline & & \multirow[t]{3}{*}{$\mathrm{O}$} & 1 & 95.64 \\
\hline & & & 2 & 97.62 \\
\hline & & & 3 & 98.21 \\
\hline & & \multirow[t]{3}{*}{$\mathrm{R}$} & 1 & 86.27 \\
\hline & & & 2 & 79.37 \\
\hline & & & 3 & 89.29 \\
\hline & \multirow[t]{6}{*}{ Without the system } & \multirow[t]{3}{*}{ G } & 1 & 90.25 \\
\hline & & & 2 & 94.62 \\
\hline & & & 3 & 97.38 \\
\hline & & \multirow[t]{3}{*}{$\mathrm{N}$} & 1 & 93.57 \\
\hline & & & 2 & 94.46 \\
\hline & & & 3 & 98.92 \\
\hline
\end{tabular}


Table D.5: Deictic Turns - Complete listing

\begin{tabular}{|c|c|c|c|c|}
\hline Cur & Experimental Condition & Trial & Lesson & Percentage of object level turns \\
\hline \multirow[t]{18}{*}{ A } & \multirow[t]{3}{*}{ All sighted } & \multirow[t]{3}{*}{ Control group 1} & 1 & 7.14 \\
\hline & & & 2 & 14.14 \\
\hline & & & 3 & 20.94 \\
\hline & \multirow[t]{6}{*}{ With the system } & \multirow[t]{3}{*}{ G } & 1 & 8.22 \\
\hline & & & 2 & 15.79 \\
\hline & & & 3 & 9.91 \\
\hline & & \multirow[t]{3}{*}{$\mathrm{N}$} & 1 & 15.86 \\
\hline & & & 2 & 18.31 \\
\hline & & & 3 & 18.85 \\
\hline & \multirow[t]{9}{*}{ Without the system } & \multirow[t]{3}{*}{ A } & 1 & 4.40 \\
\hline & & & 2 & 4.55 \\
\hline & & & 3 & 3.31 \\
\hline & & \multirow[t]{3}{*}{$\mathrm{O}$} & 1 & 2.30 \\
\hline & & & 2 & 6.02 \\
\hline & & & 3 & 2.83 \\
\hline & & \multirow[t]{3}{*}{$\mathrm{R}$} & 1 & 4.27 \\
\hline & & & 2 & 8.72 \\
\hline & & & 3 & 6.49 \\
\hline \multirow[t]{18}{*}{ B } & \multirow[t]{3}{*}{ All sighted } & \multirow[t]{3}{*}{ Control group 2} & 1 & 6.42 \\
\hline & & & 2 & 11.64 \\
\hline & & & 3 & 9.26 \\
\hline & \multirow[t]{9}{*}{ With the system } & \multirow[t]{3}{*}{ A } & 1 & 15.86 \\
\hline & & & 2 & 11.11 \\
\hline & & & 3 & 7.83 \\
\hline & & \multirow[t]{3}{*}{$\mathrm{O}$} & 1 & 10.94 \\
\hline & & & 2 & 20.63 \\
\hline & & & 3 & 8.93 \\
\hline & & \multirow[t]{3}{*}{$\mathrm{R}$} & 1 & 13.45 \\
\hline & & & 2 & 15.89 \\
\hline & & & 3 & 7.58 \\
\hline & \multirow[t]{6}{*}{ Without the system } & \multirow[t]{3}{*}{ G } & 1 & 1.19 \\
\hline & & & 2 & 3.82 \\
\hline & & & 3 & 3.81 \\
\hline & & \multirow[t]{3}{*}{$\mathrm{N}$} & 1 & 2.26 \\
\hline & & & 2 & 3.69 \\
\hline & & & 3 & 3.73 \\
\hline
\end{tabular}


Table D.6: Grades per question - Pre-test - Curriculum A

\begin{tabular}{|lrrrrrrrrrrr|}
\hline Subject/Question & $\mathbf{1}$ & $\mathbf{2}$ & $\mathbf{3}$ & $\mathbf{4}$ & $\mathbf{5}$ & $\mathbf{6}$ & $\mathbf{7}$ & $\mathbf{8}$ & $\mathbf{9}$ & $\mathbf{1 0}$ & Total \\
\hline S1 & 5 & 5 & 5 & 5 & 0 & 0 & 5 & 5 & 3 & 5 & 38 \\
S2 & 5 & 5 & 5 & 5 & 5 & 0 & 5 & 5 & 0 & 0 & 35 \\
S3 & 0 & 5 & 0 & 3 & 2 & 0 & 5 & 0 & 5 & 2 & 22 \\
N & 0 & 5 & 0 & 5 & 0 & 0 & 5 & 0 & 3 & 0 & 18 \\
G & 0 & 5 & 0 & 0 & 0 & 0 & 5 & 5 & 0 & 0 & 15 \\
S4 & 0 & 0 & 0 & 5 & 0 & 0 & 5 & 5 & 0 & 0 & 15 \\
S5 & 0 & 5 & 0 & 5 & 0 & 0 & 5 & 0 & 0 & 0 & 15 \\
S6 & 0 & 0 & 0 & 5 & 2 & 0 & 5 & 0 & 0 & 0 & 12 \\
S7 & 0 & 0 & 0 & 5 & 1 & 0 & 5 & 0 & 0 & 0 & 11 \\
S8 & 0 & 0 & 0 & 5 & 0 & 0 & 5 & 0 & 0 & 0 & 10 \\
A & 0 & 0 & 0 & 5 & 0 & 0 & 5 & 0 & 0 & 0 & 10 \\
S9 & 0 & 0 & 0 & 5 & 0 & 0 & 5 & 0 & 0 & 0 & 10 \\
S10 & 0 & 5 & 2 & 3 & 0 & 0 & 0 & 0 & 0 & 0 & 10 \\
S11 & 0 & 0 & 0 & 5 & 0 & 0 & 5 & 0 & 0 & 0 & 10 \\
S12 & 0 & 0 & 5 & 0 & 0 & 0 & 5 & 0 & 0 & 0 & 10 \\
S13 & 0 & 0 & 0 & 5 & 0 & 0 & 5 & 0 & 0 & 0 & 10 \\
S14 & 0 & 0 & 0 & 3 & 0 & 0 & 5 & 0 & 0 & 0 & 8 \\
S15 & 0 & 0 & 0 & 5 & 3 & 0 & 0 & 0 & 0 & 0 & 8 \\
O & 0 & 0 & 0 & 2 & 0 & 0 & 5 & 0 & 0 & 0 & 7 \\
S16 & 0 & 0 & 0 & 0 & 0 & 0 & 5 & 0 & 0 & 1 & 6 \\
S17 & 0 & 0 & 0 & 5 & 0 & 0 & 0 & 0 & 0 & 0 & 5 \\
R & 0 & 0 & 0 & 0 & 0 & 0 & 5 & 0 & 0 & 0 & 5 \\
S18 & 0 & 0 & 0 & 0 & 0 & 0 & 5 & 0 & 0 & 0 & 5 \\
S19 & 0 & 0 & 0 & 0 & 0 & 0 & 5 & 0 & 0 & 0 & 5 \\
\hline Grades: Pre-test Curriculum A & $($ pts & each; & total of & 50 & pts possible) \\
\hline
\end{tabular}


Table D.7: Grades per question - Post-test - Curriculum A

\begin{tabular}{|lrrrrrrrrrrr|}
\hline Subject/Question & $\mathbf{1}$ & $\mathbf{2}$ & $\mathbf{3}$ & $\mathbf{4}$ & $\mathbf{5}$ & $\mathbf{6}$ & $\mathbf{7}$ & $\mathbf{8}$ & $\mathbf{9}$ & $\mathbf{1 0}$ & \multicolumn{1}{c|}{ Total } \\
\hline S1 & 5 & 5 & 5 & 5 & 1 & 3 & 3 & 5 & 5 & 4 & 41 \\
S2 & 4 & 4 & 5 & 5 & 0 & 5 & 3 & 1 & 5 & 3 & 35 \\
S20 & 5 & 3 & 5 & 5 & 1 & 0 & 3 & 5 & 5 & 2 & 34 \\
S6 & 5 & 5 & 0 & 3 & 4 & 0 & 3 & 5 & 3 & 2 & 30 \\
S11 & 5 & 2 & 4 & 0 & 0 & 5 & 0 & 2 & 5 & 2 & 25 \\
S12 & 4 & 0 & 5 & 5 & 0 & 0 & 2 & 5 & 0 & 2 & 23 \\
S14 & 4 & 5 & 0 & 5 & 0 & 0 & 2 & 0 & 5 & 2 & 23 \\
G & 5 & 3 & 0 & 3 & 0 & 0 & 0 & 1 & 5 & 1 & 18 \\
N & 4 & 3 & 0 & 0 & 3 & 0 & 2 & 0 & 5 & 0 & 17 \\
O & 5 & 3 & 0 & 0 & 0 & 0 & 2 & 0 & 5 & 1 & 16 \\
S10 & 1 & 2 & 5 & 0 & 0 & 0 & 3 & 0 & 5 & 0 & 16 \\
R & 5 & 0 & 0 & 5 & 0 & 0 & 3 & 0 & 0 & 2 & 15 \\
S15 & 1 & 0 & 2 & 4 & 0 & 0 & 0 & 1 & 5 & 1 & 14 \\
S16 & 1 & 0 & 0 & 4 & 0 & 2 & 2 & 3 & 0 & 1 & 13 \\
S4 & 0 & 2 & 2 & 3 & 0 & 0 & 1 & 0 & 5 & 0 & 13 \\
S13 & 1 & 1 & 0 & 0 & 0 & 0 & 2 & 0 & 5 & 3 & 12 \\
S19 & 1 & 0 & 0 & 0 & 0 & 3 & 2 & 0 & 5 & 1 & 12 \\
S17 & 0 & 0 & 0 & 0 & 1 & 0 & 1 & 5 & 5 & 0 & 12 \\
S8 & 1 & 0 & 0 & 5 & 0 & 0 & 0 & 0 & 5 & 0 & 11 \\
A & 0 & 0 & 0 & 0 & 0 & 3 & 2 & 0 & 5 & 0 & 10 \\
S19 & 0 & 0 & 0 & 3 & 0 & 0 & 1 & 0 & 5 & 0 & 9 \\
S5 & 4 & 0 & 0 & 0 & 0 & 0 & 3 & 0 & 0 & 0 & 7 \\
\hline Grades: Post-test Curriculum A ( pts each; total of 50 & pts possible) \\
\hline
\end{tabular}


Table D.8: Grades per question - Pre-test - Curriculum B

\begin{tabular}{|lrrrrrrrrrrr|}
\hline Subject/Question & $\mathbf{1}$ & $\mathbf{2}$ & $\mathbf{3}$ & $\mathbf{4}$ & $\mathbf{5}$ & $\mathbf{6}$ & $\mathbf{7}$ & $\mathbf{8}$ & $\mathbf{9}$ & $\mathbf{1 0}$ & \multicolumn{1}{c|}{ Total } \\
\hline S1 & 5 & 5 & 5 & 0 & 0 & 5 & 0 & 5 & 0 & 5 & 30 \\
S6 & 5 & 3 & 5 & 0 & 0 & 5 & 0 & 5 & 5 & 0 & 28 \\
G & 5 & 3 & 5 & 0 & 0 & 5 & 0 & 5 & 0 & 0 & 23 \\
S7 & 5 & 5 & 0 & 5 & 0 & 3 & 0 & 5 & 0 & 0 & 23 \\
S11 & 5 & 0 & 5 & 0 & 0 & 3 & 0 & 5 & 0 & 0 & 18 \\
O & 5 & 0 & 0 & 0 & 0 & 5 & 0 & 5 & 0 & 0 & 15 \\
S2 & 5 & 0 & 5 & 0 & 0 & 5 & 0 & 0 & 0 & 0 & 15 \\
S4 & 5 & 0 & 5 & 0 & 0 & 0 & 0 & 3 & 0 & 0 & 13 \\
R & 3 & 0 & 0 & 0 & 0 & 0 & 0 & 5 & 0 & 5 & 13 \\
S8 & 3 & 0 & 5 & 0 & 0 & 3 & 0 & 0 & 0 & 0 & 11 \\
S3 & 5 & 5 & 0 & 0 & 0 & 0 & 0 & 0 & 0 & 0 & 10 \\
S10 & 5 & 0 & 0 & 0 & 0 & 0 & 0 & 5 & 0 & 0 & 10 \\
S5 & 5 & 0 & 0 & 0 & 0 & 3 & 0 & 0 & 0 & 0 & 8 \\
S9 & 0 & 0 & 5 & 0 & 0 & 3 & 0 & 0 & 0 & 0 & 8 \\
\hline Grades: Post-test Curriculum B $(5$ & pts & each; total of 50 pts possible $)$ \\
\hline
\end{tabular}


Table D.9: Grades per question - Post-test - Curriculum B

\begin{tabular}{|lrrrrrrrrrrr|}
\hline Subject/Question & $\mathbf{1}$ & $\mathbf{2}$ & $\mathbf{3}$ & $\mathbf{4}$ & $\mathbf{5}$ & $\mathbf{6}$ & $\mathbf{7}$ & $\mathbf{8}$ & $\mathbf{9}$ & $\mathbf{1 0}$ & \multicolumn{1}{r|}{ Total } \\
\hline S14 & 5 & 5 & 5 & 5 & 5 & 5 & 5 & 5 & 5 & 5 & 50 \\
S13 & 3 & 5 & 3 & 5 & 3 & 3 & 5 & 5 & 5 & 0 & 37 \\
S1 & 5 & 5 & 2 & 2 & 5 & 0 & 3 & 5 & 5 & 0 & 32 \\
G & 4 & 2 & 2 & 1 & 3 & 3 & 3 & 5 & 5 & 0 & 28 \\
S6 & 5 & 5 & 2 & 0 & 2 & 2 & 1 & 5 & 5 & 0 & 27 \\
S12 & 5 & 2 & 0 & 5 & 2 & 2 & 3 & 5 & 0 & 0 & 24 \\
N & 5 & 0 & 0 & 0 & 0 & 2 & 3 & 3 & 5 & 0 & 18 \\
S5 & 0 & 4 & 2 & 0 & 0 & 0 & 5 & 5 & 0 & 0 & 16 \\
S11 & 4 & 0 & 1 & 0 & 0 & 0 & 5 & 5 & 0 & 0 & 15 \\
R & 3 & 1 & 0 & 0 & 0 & 0 & 0 & 5 & 5 & 0 & 14 \\
O & 5 & 2 & 0 & 2 & 0 & 0 & 0 & 5 & 0 & 0 & 14 \\
S8 & 5 & 0 & 0 & 0 & 2 & 2 & 0 & 5 & 0 & 0 & 14 \\
S7 & 4 & 5 & 0 & 0 & 0 & 0 & 0 & 0 & 5 & 0 & 14 \\
S9 & 4 & 3 & 2 & 0 & 0 & 0 & 0 & 5 & 0 & 0 & 12 \\
S15 & 5 & 3 & 0 & 0 & 2 & 2 & 0 & 0 & 0 & 0 & 12 \\
S4 & 5 & 5 & 0 & 0 & 0 & 2 & 0 & 0 & 0 & 0 & 12 \\
S3 & 5 & 0 & 0 & 0 & 0 & 5 & 0 & 0 & 0 & 0 & 10 \\
S2 & 2 & 0 & 0 & 0 & 0 & 2 & 3 & 2 & 0 & 0 & 9 \\
A & 2 & 2 & 0 & 0 & 0 & 0 & 0 & 3 & 0 & 0 & 7 \\
\hline Grades: Post-test Curriculum B & 5 & pts & each; total of 50 & pts possible $)$ \\
\hline
\end{tabular}

